Qhiversidad Yacional de Sla OPlata

Fracultad de Ingeniería

Orea OSepartamental Ingeniería Química

Margo 2010

\title{
Simulación Numérica de Problemas de Transferencia de Energía en Alimentos de Geometría Irregular Utilizando el Método de los Elementos Finitos
}

\section{Victoria Santos}

Tesis presentada para el grado de Doctor en Ingeniería

Director: Dra. N. Zaritzky

Co-Director: Dra. A. Califano 
M Yosé Sebastián, mi hijo. 
Esta tesis se realizó en el Centro de Investigación y Desarrollo en Criotecnología de Alimentos (CIDCA), organismo dependiente de la Facultad de Ciencias Exactas de la Universidad Nacional de la Plata y del Consejo Nacional de Investigaciones Científicas y Técnicas (CONICET), bajo la dirección de la Dra. Noemí Zaritzky y la Dra. Alicia Califano.

El presente trabajo de Tesis doctoral fue financiado a través de una beca de Posgrado tipo I otorgada por el CONICET a partir del 1 de Abril del año 2005. 
Un agradecimiento especial a mi marido, Martín Ducos, que siempre me acompañó y colaboró para que pueda concretar todas mis metas personales y profesionales.

A mis suegros, especialmente mi suegra, que siempre está presente con su termo y mate lavado.

A mis cuñados, David y Mariano, y mí cuñada Ailín por haberme aguantado y darme una mano siempre que lo necesité.

Un agradecimiento especial a la tía Elena, Néstor y Daniela por ser tan dulces con Seba y estar siempre presente.

A Gabriel por aguantarme en la oficina, y estar siempre dispuesto a dar una ayuda.

Un especial agradecimiento a Victoria Vampa que colaboró con el entendimiento de los fundamentos matemáticos y por ser una persona con mucha paciencia, siempre dispuesta a explicar un problema de mil formas distintas.

Además, un agradecimiento destacado a las personas que me formaron y acompañaron en estos años y durante mis años en la facultad, Alicia y Mimí.

$\mathrm{Y}$ muy especialmente a mis padres y a mi hermana por todo el esfuerzo y paciencia que han tenido conmigo y su apoyo incondicional. 
Parte de los resultados obtenidos de la presente Tesis fueron publicados en:

M. V. Santos, N. Zaritzky, A. Califano (2010). A control strategy to assure safety conditions in the thermal treatment of meat products using a numerical algorithms. Food Control ISSN=0956-7135, editorial Elsevier. Vol 21 pp 191-197.

M. V. Santos, N. Zaritzky, A. Califano (2008). Modeling heat transfer and inactivation of Escherichia coli $0157: \mathrm{H} 7$ in precooked meat products in Argentina using the finite element method. Meat Science ISSN=03091740, editorial Elsevier. Vol 79 Issue 3 pp 595-602.

M. V. Santos, N. Zaritzky, A. Califano, V. Vampa (2008). Numerical Simulation of the Heat Transfer in Three Dimensional Geometries. Mecánica Computacional ISSN=1666-6070. Vol XXVII No 21 Heat Transfer (C) editorial Asociación Argentina de Mecánica Computacional (AMCA), pp 1705-1718.

M. V. Santos, V. Vampa, A. Califano, N. Zaritzky. Numerical simulations of chilling and freezing processes applied to bakery products in irregularly 3D geometries. Trabajo aceptado (13/3/2010) para su publicación en la Revista Journal of Food Engineering. 


\section{Nomenclatura}

CG matriz global de capacitancia

Cp calor específico $(\mathrm{J} /(\mathrm{kg} \mathrm{K}))$

$\mathrm{Cp}_{\mathrm{ap}} \quad$ calor específico aparente $(\mathrm{J} /(\mathrm{kg} \mathrm{K}))$

$D_{\text {ref }} \quad$ tiempo de reducción decimal $(\mathrm{min})$

E función de Kirchhoff $(\mathrm{W} / \mathrm{m})$

FG vector global de esfuerzos

h coeficiente de transferencia de calor en la interfase $\left(\mathrm{W} / \mathrm{m}^{2}{ }^{\circ} \mathrm{C}\right)$

$\mathrm{H} \quad$ vector cuyos elementos son las funciones de interpolación

$\mathrm{H}_{\mathrm{a}} \quad$ entalpía volumétrica $\left(\mathrm{J} / \mathrm{m}^{3}\right)$

J matriz jacobiana

k conductividad térmica $\left(\mathrm{W} / \mathrm{m}^{\circ} \mathrm{C}\right)$

KG matriz global de conductancia

MG matriz convectiva global

$\mathrm{m}_{\mathrm{w}} \quad$ masa de agua del baño $(\mathrm{kg})$

n número de embutidos

$\mathrm{nr}, \mathrm{nz} \quad$ componentes del vector normal unitario exterior (coord. cilíndricas)

$\mathrm{nx}, \mathrm{ny}, \mathrm{nz}$ componentes del vector normal unitario exterior (coord. cartesianas)

$\mathrm{N}$ número de microorganismos (UFC/g = unidades formadoras de colonia/gramo)

$\mathrm{N}_{0} \quad$ número inicial de microorganismos (UFC/g)

q flujo de calor $\left(\mathrm{W} / \mathrm{m}^{2}\right)$

$Q_{\mathrm{ef}} \quad$ calor efectivo entregado al sistema (W)

$Q_{\text {perdido }} \quad$ calor perdido al medio externo (W)

$Q_{\text {producto }} \quad$ calor entregado a un embutido (W)

$Q_{\text {quemador }}$ poder calorífico del quemador (W) 


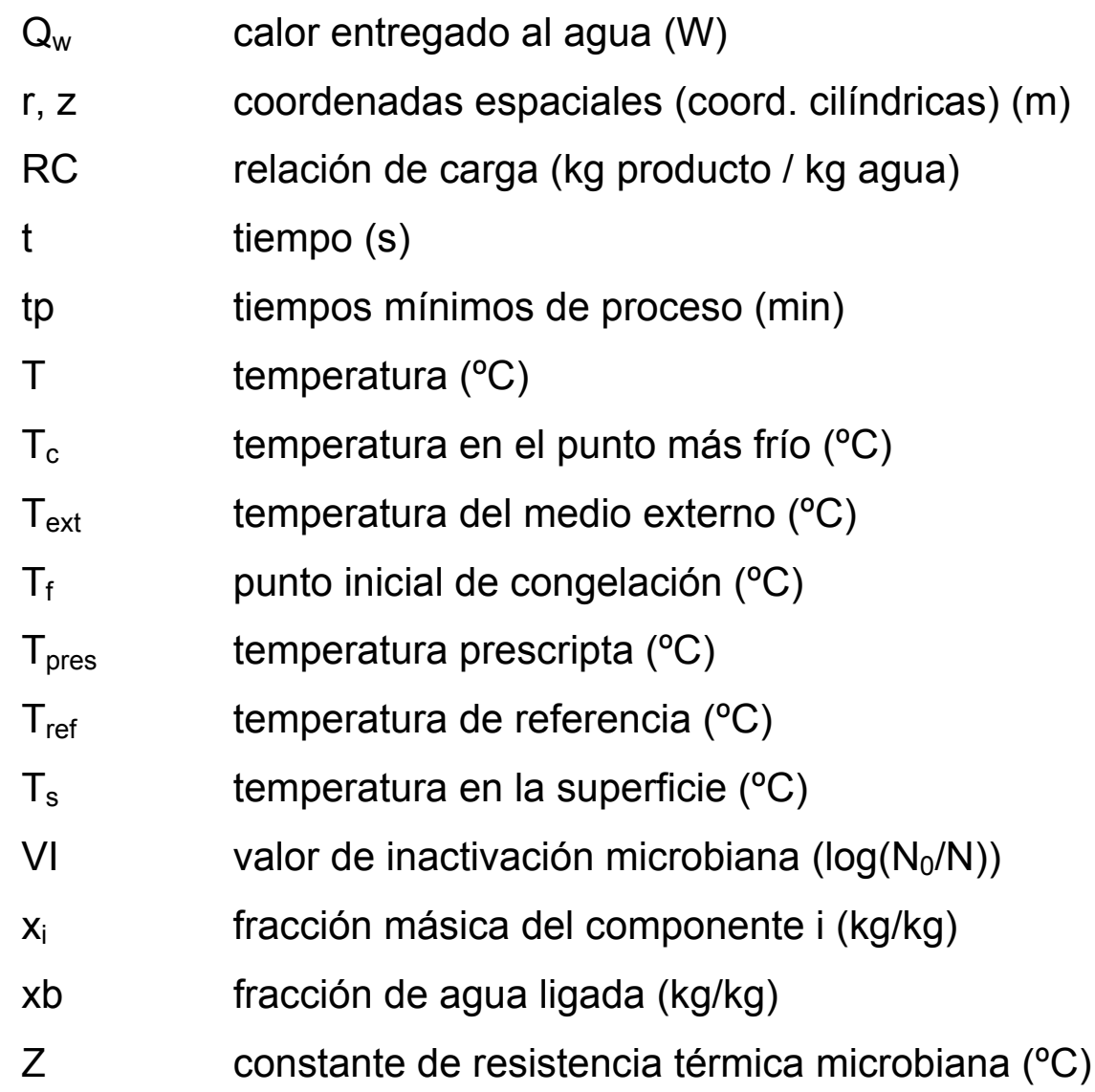

Letras griegas

$\Delta \mathrm{t} \quad$ incremento del tiempo (s)

$\delta \Omega \quad$ interfase del dominio

$\varepsilon \quad$ residuo $\left(\mathrm{W} / \mathrm{m}^{3}\right)$

$\rho \quad$ densidad $\left(\mathrm{kg} / \mathrm{m}^{3}\right)$

$\Omega \quad$ dominio

Subíndices

$0 \quad$ inicial

1, 2, 3 dominio

e elemento 

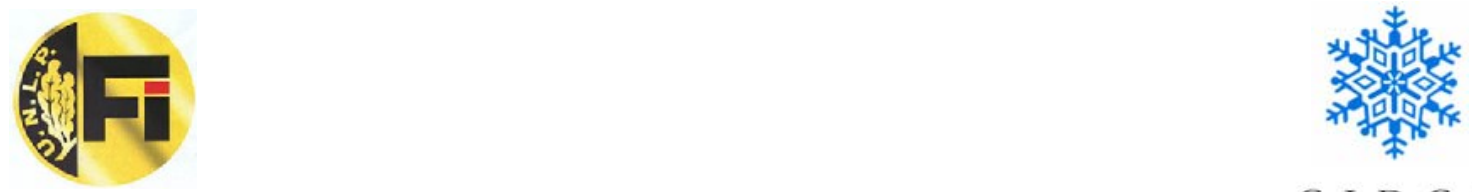

C I D C A

$\begin{array}{ll}\text { e1 } & \text { elemento de borde } \\ \text { h } & \text { hielo } \\ \text { j } & \text { nivel temporal } \\ \text { k } & \text { componente } \\ \text { w } & \text { agua }\end{array}$

Supraíndices

t traspuesto 
Capítulo 1 


\section{Introducción}

La simulación computacional aplicada a procesos de transferencia de calor en alimentos es una herramienta fundamental para el desarrollo de nuevas tecnologías y metodologías de trabajo a lo largo de toda la cadena de elaboración de productos. La ventaja principal radica en la reducción de tiempos y costos respecto a la realización de experimentos con prototipos en escala industrial, que requieren de personal entrenado para su correcta ejecución.

La simulación computacional también aporta información valiosa para empresas del sector productivo alimentario que se encuentran en una etapa de adquisición de equipos tales como congeladores, cámaras de refrigeración, etc., ya que permite conocer los tiempos de proceso y comparar entre distintos modelos y diseños.

La utilización de métodos experimentales para la evaluación de las condiciones que permiten optimizar procesos de transferencia de energía en alimentos, implicarían barrer amplios rangos de temperatura del medio, de coeficientes transferencia de calor en la interfase, temperaturas iniciales, etc. Esto conduce a un elevado número de experimentos, con un alto costo, que además consumen tiempo e interfieren en el normal funcionamiento de la planta.

Un modelo numérico que representa el proceso adecuadamente utiliza al programa computacional como un prototipo virtual (Martins, 2006) en el cual la modificación de parámetros y condiciones resulta fácil de implementar, logrando resultados en forma rápida con un error aceptable. La simulación computacional aporta información detallada y continua, tanto en función del tiempo como de las coordenadas espaciales. Sin embargo hay que tener en cuenta que la simulación numérica resulta útil siempre y cuando el modelo haya sido validado experimentalmente, alimentando al código computacional propiedades termofísicas y coeficientes de transferencia de interfase 
determinados previamente, ya sea en forma experimental o a partir de correlaciones de literatura.

Existen en el mercado software o simuladores comerciales como COMSOL, ANSYS, ABAQUS, etc. que utilizan el método de elementos finitos, cuyas licencias se pueden adquirir a un costo aceptable para las universidades. Estos paquetes permiten realizar simulación de transferencia de energía en procesos industriales que dan resultados aceptables en muchas aplicaciones. Sin embargo, existen una gran cantidad de problemas que requieren formulaciones específicas por las cuales debe modificarse el software, lo que no siempre es posible. Por esta razón el hecho de desarrollar software o programas computacionales abiertos para problemas determinados, permite abordar problemas más complejos a diferencia de la utilización de paquetes comerciales como cajas negras.

\subsection{Modelado matemático}

El modelado matemático de procesos en ingeniería de alimentos implica resolver en una gran mayoría de los casos, ecuaciones diferenciales a derivadas parciales. La ecuación diferencial que representa la ecuación de conducción de calor (Carslaw y Jaeger, 1959):

$$
\rho \mathrm{Cp} \frac{\partial \mathrm{T}}{\partial \mathrm{t}}=\nabla \cdot(\mathrm{k} \nabla \mathrm{T})
$$

siendo $\rho$ la densidad, $\mathrm{Cp}$ calor específico, $\mathrm{k}$ conductividad térmica, $\mathrm{T}$ la temperatura, y t el tiempo.

Las soluciones analíticas a dicha ecuación existen para geometrías de forma regular como esferas, cilindros finitos o infinitos, placas planas y paralelepípedos y fueron ampliamente utilizadas en procesos de transferencia de calor (Singh y Heldman, 1981) y aplicados por analogía a transferencia de 


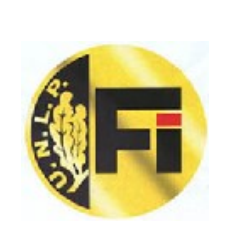

materia (Rodríguez y Zaritzky, 1986). En la actualidad se utilizan en muchos casos para obtener propiedades térmicas de alimentos (Erdogdu, 2005). Las soluciones analíticas de las ecuaciones diferenciales a derivadas parciales, son útiles pero resultan insuficientes cuando se requiere el conocimiento cuantitativo en sistemas complejos, donde por ejemplo las propiedades termofísicas no se mantienen constantes, o cuando las geometrías son irregulares etc. Los modelos numéricos, por ejemplo las diferencias finitas, el método de la grilla ajustada a los contornos, y los elementos finitos son más adecuados para resolver dichos problemas (Pham, 2006, 2008; Califano y Zaritzky, 1993, 1997).

El método de las diferencias finitas se utiliza para modelar procesos en ingeniería de alimentos en geometrías regulares: esferas, cilindros, paralelepípedos (Holtz y Skjoldenbrand, 1986; Pham, 1991; Tocci y Mascheroni, 1995; Graiver y col., 2007).

El método de los elementos finitos (MEF) es útil para simular procesos donde las geometrías no pueden representarse utilizando una grilla regular. Sin embargo esta versatilidad se gana a expensas de mayores requerimientos en cuanto memoria, tiempos de ejecución de máquina y criterios para asegurar la estabilidad y no-oscilación del método (Cleland y col., 1984). El método de elementos finitos ha sido utilizado en diversos problemas de transferencia de calor y/o materia en la industria de alimentos tales como calentamiento (Naveh y col., 1983; Nicolai y col., 1995) y enfriamiento (Arce y col., 1983), cocción en hornos (Chen y col., 1999), congelación (Rabellato y col., 1987; Cleland y col., 1987), descongelación (Abdalla y Singh, 1985), y secado (Lamauro y Bakshi, 1985).

\subsection{Problemática planteada desde el sector industrial}

La industria alimentaria muestra un notorio interés en resolver problemas de optimización y control de sus procesos. En algunos sectores tales como el 
cárnico y de panificados, existe una tendencia creciente en obtener productos semi o totalmente elaborados (alimentos RTE, en ingles "ready to eat"); el valor del mercado de productos RTE en Europa se pronostica que alcance los 13.4 miles de millones de euros para el año 2010 (An., 2008). Normalmente los alimentos pre-elaborados no tienen geometrías regulares (como esferas, cubos, placas planas, etc.) sino que presentan diversas formas geométricas, frecuentemente irregulares. Resolver las problemáticas industriales teniendo en cuenta la geometría irregular del producto ayuda a que los resultados obtenidos estén ajustados a la realidad. El método de los elementos finitos, como se dijo anteriormente, es el más adecuado para encarar los problemas de interés del sector, dada su capacidad para resolver problemas de geometría irregular.

Se presentan a continuación algunos ejemplos de la industria alimentaria donde resulta importante la resolución del problema de transferencia de energía en dominios de geometría irregular.

Uno de los objetivos más importantes dentro de la industria de preelaborados cárnicos es lograr la inocuidad del producto final, de tal manera de minimizar los riesgos de supervivencia de microorganismos patógenos, afectando lo menos posible los aspectos de calidad. Entre las problemáticas encontradas en las plantas elaboradoras de embutidos se encuentra el inadecuado o insuficiente tratamiento térmico que sufren los productos. Los embutidos pueden considerarse desde el punto de vista geométrico como cuerpos irregulares bidimensionales de revolución.

Según la clasificación del Código Alimentario Argentino (CAA, 2005) la morcilla es un embutido cocido realizado a base de sangre, que sufre un proceso de cocción en agua. Según un estudio realizado en la ciudad de La Plata (Oteiza y col., 2003), se detectó la presencia de microorganismos patógenos en muestras estudiadas de distintos establecimientos comerciales. La existencia de dichos microorganismos se debe a varias causas, una de las cuales es el insuficiente o inadecuado tratamiento térmico del producto. Esta problemática originó el interés por el proceso, planteando el desarrollo de los 


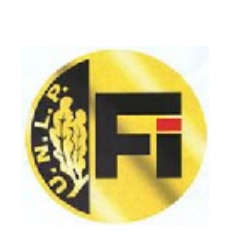

siguientes aspectos: optimización del proceso térmico, considerando la geometría irregular del producto y las condiciones industriales (volumen del medio calefactor, volumen de producto, energía entregada por el quemador, e inercia térmica del sistema).

Otro problema planteado desde el sector productivo está relacionado con el proceso de refrigeración de productos semi terminados de composición heterogénea, como es el caso de empanadas, compuesto por masa cruda y un relleno. En la fabricación de empanadas a escala industrial se elaboran los productos en una planta para luego ser refrigerados y/o congelados para su posterior transporte a los distintos locales de venta al público. Allí los productos son horneados de acuerdo a la demanda ofreciendo a los consumidores un producto fresco "recién horneado".

La problemática planteada desde esta industria radica entonces en conocer los tiempos de refrigeración para distintas condiciones de proceso, como puede ser en túnel o cámaras, temperaturas iniciales de producto, etc. Este problema corresponde a una geometría tridimensional irregular del producto con regiones bien diferenciadas que tienen distintas propiedades termofísicas, por ejemplo en el caso de rellenos a base de carne; fase farinácea y fase cárnea.

El sector de elaboración de panificados también en los últimos años tuvo una fuerte demanda de productos semi terminados. Al centralizar los establecimientos de elaboración de productos panificados se vieron reducidos los costos relacionados con las pérdidas económicas a causa del descarte de productos terminados que no se comercializaban en la misma jornada, el mantenimiento de grandes locales con personal especializado y equipamientos de gran consumo y costos. A través de la cadena de distribución, estos productos congelados son llevados a los distintos establecimientos de venta donde se almacenan y según la demanda se preparan para el proceso de horneado. Esta tecnología llamada BOT (en inglés "Bake Off Technologies") minimiza los costos de mantenimiento de locales ya que la venta del producto 
recién horneado se realiza en establecimientos pequeños con mínimos requerimientos (cámaras de descongelación y leudado, horno) y personal poco entrenado. El mercado de BOT crece a un ritmo del $10 \%$ anual y se evidencia fuertemente el traspaso de la elaboración tradicional de panificados al BOT (LeBail y Goff, 2008).

En este caso la congelación de un producto panificado de amplia demanda (medialunas-croissant) resulta un problema de interés desde el punto de vista de la transferencia de energía donde se buscan establecer los tiempos de congelación teniendo en cuenta su geometría irregular tridimensional y el cambio en las propiedades térmicas del alimento durante su congelación, constituyendo éste un problema complejo que además incluye el cambio de fase.

Los tres ejemplos anteriormente expuestos corresponden a problemas de transferencia de energía en geometrías irregulares con propiedades termofísicas $(\rho, C p, k)$ constantes o variables con la temperatura según el proceso, y con cambio de fase en el caso de congelación constituyendo en este último caso un problema no lineal.

En el presente trabajo de Tesis se desarrolla la resolución numérica de estos sistemas mediante la utilización del método de los elementos finitos con grado de dificultad matemática creciente en función de la no linealidad del problema.

En el método de los elementos finitos (MEF) el dominio se divide en pequeños elementos en cuyos vértices se ubican los nodos. La temperatura en cualquier punto del dominio se obtiene a través de funciones de interpolación y las temperaturas nodales ( $\mathrm{T} 1, \mathrm{~T} 2$, y $\mathrm{T} 3$ ) del elemento que contiene a dicho punto (Ec. 1.2). Estas funciones de interpolación se las denomina funciones de forma y son dependientes de la posición (h1, h2, y h3). (Fig. 1.1)

$\mathrm{T}=\mathrm{T} 1 \cdot \mathrm{h} 1+\mathrm{T} 2 \cdot \mathrm{h} 2+\mathrm{T} 3 \cdot \mathrm{h} 3$ 


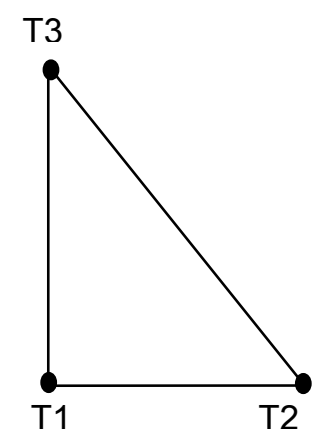

Fig. 1-1 Elemento bidimensional triangular en cuyos vértices se encuentran los nodos

En el Capítulo 2 de la Tesis se desarrollará en detalle el tipo de funciones de interpolación utilizadas para calcular la variable $\mathrm{T}$.

La discretización del dominio continuo debe hacerse con elementos que mantengan lo mejor posible la figura irregular del dominio inicial y que a medida que aumenta el refinamiento (aumentando el número de elementos) se mejore tanto el grado de aproximación del dominio como la solución numérica. A su vez se debe tener en cuenta que un excesivo refinamiento produce una acumulación de errores y mayor esfuerzo computacional, de modo que existe una relación de compromiso entre el grado de precisión del resultado numérico y tiempos de ejecución de máquina. Debido a que las temperaturas en el dominio son aproximaciones y no valores exactos, en general la ecuación de transferencia de calor no será valida en cada elemento, por ende a este desfasaje o diferencia se lo denomina residuo (Ec. 1.3).

$\varepsilon=\rho \operatorname{Cp} \frac{\partial \mathrm{T}}{\partial \mathrm{t}}-\nabla \cdot(\mathrm{k} \nabla \mathrm{T})$

El método de elementos finitos utiliza una formulación variacional donde se exige que la integral del residuo ponderada en los nodos sea cero; de esta manera la energía se conserva en promedio en todos los elementos. Al utilizar las funciones de forma tanto para ponderar la integral como para representar la 
temperatura se aplica el método de Galerkin (Zienkiewicz y Taylor, 1994a, 1994b) con el método de elementos finitos.

$\int \varepsilon \cdot$ hi $d \Omega=\int\left(\rho \operatorname{Cp} \frac{\partial \mathrm{T}}{\partial \mathrm{t}}-\nabla \cdot(\mathrm{k} \nabla \mathrm{T})\right) \cdot$ hid $\Omega=0$

donde $\Omega$ es el dominio de integración y hi con i=1, 2 o 3 son las funciones de interpolación. Si se realiza una integración por partes y se emplea el teorema de la divergencia se incorporan las condiciones de contorno de tipo convectiva $(\mathrm{q}=\mathrm{h} \Delta \mathrm{T})$. El sistema resultante, que corresponde a la discretización espacial, representa un sistema de $\mathrm{N}$ ecuaciones diferenciales ordinarias, donde $\mathrm{N}$ es el número de nodos de la malla. En el Capítulo 2 de la presente Tesis se explicará con detalle el desarrollo del método por elementos finitos en sistemas bidimensionales.

Una de las características más importantes del método radica en que la formulación variacional satisface el balance microscópico de energía necesitando restricciones más débiles, que las que exige el problema original. Estas restricciones que implican que exista la integral de las derivadas parciales de primer orden elevadas al cuadrado (por ejemplo $\int\left(\frac{\partial T}{\partial x}\right)^{2} d x$ ), son menos fuertes que las del problema original, en el cual intervienen derivadas parciales de segundo orden, $\left(\frac{\partial^{2} T}{\partial x^{2}}\right)$ (Zienkiewicz y Taylor, 1994a, 1994b, Segerlind, 1984; Becker y col., 1983).

\subsection{Problemas de transferencia de calor sin cambio de fase (problemas lineales)}

Los simuladores comerciales tienen la ventaja de tener una interfase 
atractiva y simple para el usuario el cual puede ingresar los parámetros del proceso y enfocarse en los resultados que el simulador le brinda. Además pueden importar archivos CAD (Computer Aided Design) generados en programas específicos de diseño y dibujo que permiten generar el mallado de geometrías irregulares. Constituyen herramientas muy potentes y adecuadas por su velocidad de cálculo para simular todo tipo de problemas lineales bi y tridimensionales como calentamientos y enfriamientos donde las propiedades físicas del alimento no dependan fuertemente con la temperatura.

Si bien son aptos para simular infinidad de problemas de transferencia de calor y presentan una gráfica de postprocesamientos variada, al ser paquetes cerrados, resulta a veces dificultoso ubicar dónde y cuáles son los problemas de convergencia del método numérico utilizado. Aún en los casos donde se puede obtener un código computacional que describe en sentencias el problema simulado, al estar escrito y codificado por dicho software en estructuras y nomenclaturas específicas, resulta a veces difícil implementar acoplamientos específicos, por ejemplo, cinéticas de inactivación en el punto más frío del alimento, con otras variables y ecuaciones que intervienen en el proceso, como cuando la temperatura del medio externo es variable y debe ser acoplada por ejemplo a la resolución de los balances macroscópicos de energía.

\subsection{Problemas de transferencia de calor con cambio de fase (problemas no lineales)}

El proceso de congelación es uno de los más importantes dentro de la ingeniería de alimentos en donde el estudio detallado del fenómeno de cambio de fase en el producto es fundamental para realizar el correcto diseño y optimización de plantas de congelación (Rabellato y col., 1978). Por consiguiente es necesario contar con predicciones precisas de los tiempos de 


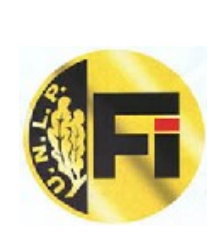

congelación, velocidades de congelación y distribución de temperaturas en el alimento.

El análisis del proceso de transferencia de energía en la congelación es un proceso complejo con cambio de fase, en el que las propiedades termofísicas varían fuertemente con la temperatura a través de los cambios de fracción de agua congelada.

\subsubsection{Propiedades térmicas durante el cambio de fase (temperatura inicial de congelación)}

En los alimentos en general el agua es el componente que se encuentra en mayor proporción, por ende cuando la temperatura desciende por debajo de $0^{\circ} \mathrm{C}$ comienza la formación de hielo. El alimento congelado se puede considerar formado por cristales de hielo y una solución con componentes sólidos en una fase acuosa. A medida que avanza la congelación del alimento la solución en equilibrio se encuentra más concentrada en solutos disminuyendo la temperatura de equilibrio a lo largo de la congelación. El proceso de congelación entonces ocurre en un rango de temperaturas y no a una temperatura única como en el caso del agua pura.

Existen en literatura varias correlaciones empíricas y modelos matemáticos para la determinación de puntos iniciales de congelación de alimentos según el contenido de agua (Salvadori y Mascheroni, 1991; Levy, 1979; Chang y Tao, 1981). Experimentalmente se puede determinar el punto inicial de congelación a través de curvas de congelación (Fennema y col., 1973) o curvas de flujo de calor obtenidas utilizando un Calorímetro Diferencial de Barrido (Rahman, 2005). La determinación del punto inicial de congelación, $\mathrm{T}_{\mathrm{f}}\left({ }^{\circ} \mathrm{C}\right)$, es fundamental para realizar cálculos de estimación de propiedades termofísicas de alimento y por consiguiente el correcto diseño de equipos de congelación. 


\subsubsection{Calor específico aparente}

El calor específico se define como el calor necesario para aumentar la temperatura de una unidad de masa de un alimento en un grado centígrado. El calor específico del agua $\mathrm{Cp}_{\mathrm{w}}=4.18 \mathrm{~J} / \mathrm{g}{ }^{\circ} \mathrm{C}$ a $20^{\circ} \mathrm{C}$ es alto comparado con otras sustancias. Esta propiedad está relacionada con los enlaces puentes de hidrógeno que se crean entre las moléculas de agua, los cuales hacen que se unan electrostáticamente. Esta propiedad del agua influye también en el calor latente de fusión o congelación $\mathrm{L}$, el cual es alto comparado con otras sustancias (334 J/g a 1 bar y $0^{\circ} \mathrm{C}$ ). Debido a estos valores de calor específico y calor latente la cantidad de calor requerida para congelar o descongelar un alimento aumenta con el contenido de agua del mismo.

El calor específico se puede determinar experimentalmente utilizando métodos calorimétricos en los rangos de temperatura donde no existe cambio de fase, denominándose calor específico sensible. El calor específico se puede calcular mediante un modelo aditivo (Choi y Okos, 1986) que considera el aporte de cada componente al calor específico del alimento (Ec. (1.5))

$\mathrm{Cp}=\sum \mathrm{x}_{\mathrm{k}} \cdot \mathrm{Cp_{ \textrm {k } }}$

donde $\mathrm{Cp}$ es el calor específico del alimento, $x_{k}$ es la fracción másica de los componentes (agua, proteína, carbohidratos, lípido, cenizas, etc.), y $\mathrm{Cp}_{\mathrm{k}}$ el calor específico de cada componente.

$\mathrm{Si}$ el rango de temperaturas comprende el cambio de fase es necesario tener en cuenta el aporte del calor latente en el rango de fusión del alimento de acuerdo a la fracción de hielo que se forma, el cual se denomina calor específico aparente. El calor específico aparente puede obtenerse mediante DSC considerando el pico de fusión en el rango de temperaturas correspondiente. También puede calcularse mediante la siguiente formula (Miles, 1983): 
$C p_{a p}(T)=C p_{s} \cdot\left(1-x_{w}\right)+C p_{w} \cdot x_{w} \cdot \frac{T_{f}}{T}+C p_{h} \cdot\left(1-\frac{T_{f}}{T}\right) x_{w}-L \cdot x_{w} \cdot \frac{T_{f}}{T^{2}}$

donde $\mathrm{Cp}_{\mathrm{s}}$ es el calor específico del sólido y la fracción de hielo que se estima mediante la ecuación 1.7 .

$$
x_{h}=x_{w}\left(1-\frac{T_{f}}{T}\right)
$$

Si consideramos la cantidad de agua ligada o no congelable, la fracción de hielo $x_{h}$ se estima (Miles, 1974) de la siguiente forma:

$$
x_{h}=\left(x_{w}-x b\right) \cdot\left(1-\frac{T_{f}}{T}\right)
$$

donde $\mathrm{xb}$ es la fracción de agua no congelable a una temperatura de $-40{ }^{\circ} \mathrm{C}$ (Fennema, 1993). Esta fracción de agua si se determina por DSC o NMR (resonancia magnética nuclear) es del orden del $20 \%$ del total de agua que tiene el alimento, dado que se considera el agua en estado vítreo (van der Sman y Boer, 2005).

En la Fig. 1-2 se puede observar una curva típica de calor específico en función de la temperatura en un alimento.

\subsubsection{Conductividad térmica}

La conductividad térmica del alimento también está fuertemente determinada por el contenido de hielo y agua. La conductividad del agua en estado líquido es $0.554 \mathrm{~W} / \mathrm{m}{ }^{\circ} \mathrm{C}$ comparado con la del hielo que es 2.25 $\mathrm{W} / \mathrm{m}^{\circ} \mathrm{C}\left(\mathrm{a}^{\circ}{ }^{\circ} \mathrm{C}\right)$, estando los carbohidratos, proteínas y lípidos en un rango de 0.17-0.20 W/m ${ }^{\circ} \mathrm{C}$ (Pham, 2008). 


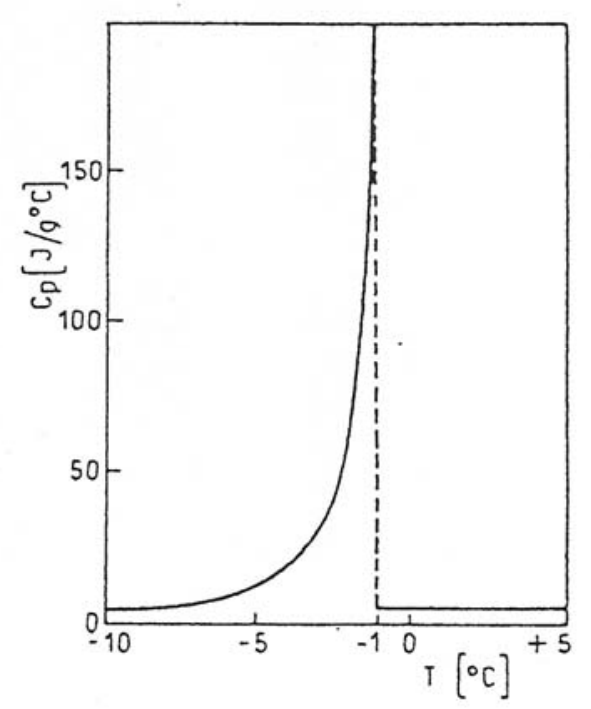

Fig. 1-2 Calor específico aparente en función de la temperatura en el rango de congelación

En la Fig. 1-3 se puede observar la conductividad de un alimento con distinto contenido de agua en función de la temperatura.

Existen distintos modelos para estimar la conductividad térmica de alimentos congelados que tienen en cuenta la estructura heterogénea del alimento. Los modelos pueden ser considerando flujo calórico paralelo o perpendicular a las fibras (aplicable al caso de tejidos). También se puede considerar como esferas dispersas en una fase continua según la disposición de los componentes dentro del alimento (modelo de Maxwell-Eucken (Miles y col. 1983; Choi y Okos, 1986).

\subsubsection{Densidad}

La densidad también sufre cambios significativos durante la congelación de alimentos (Fig. 1-4), dado que el agua se expande un $10 \%$ por debajo del punto de inicial de congelación (disminuye la densidad) generando un stress térmico-mecánico importante. 
C I D C A

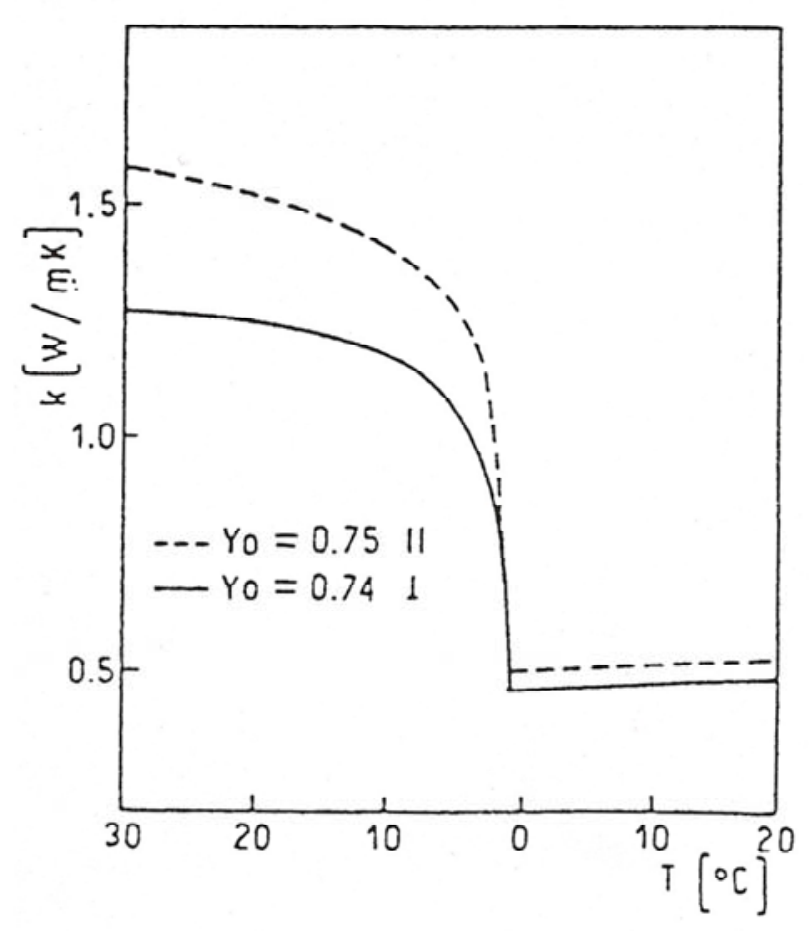

Fig. 1-3 Efecto de la temperatura en la conductividad térmica de carne con distinto contenido de agua, con flujo calórico paralelo y perpendicular a las fibras

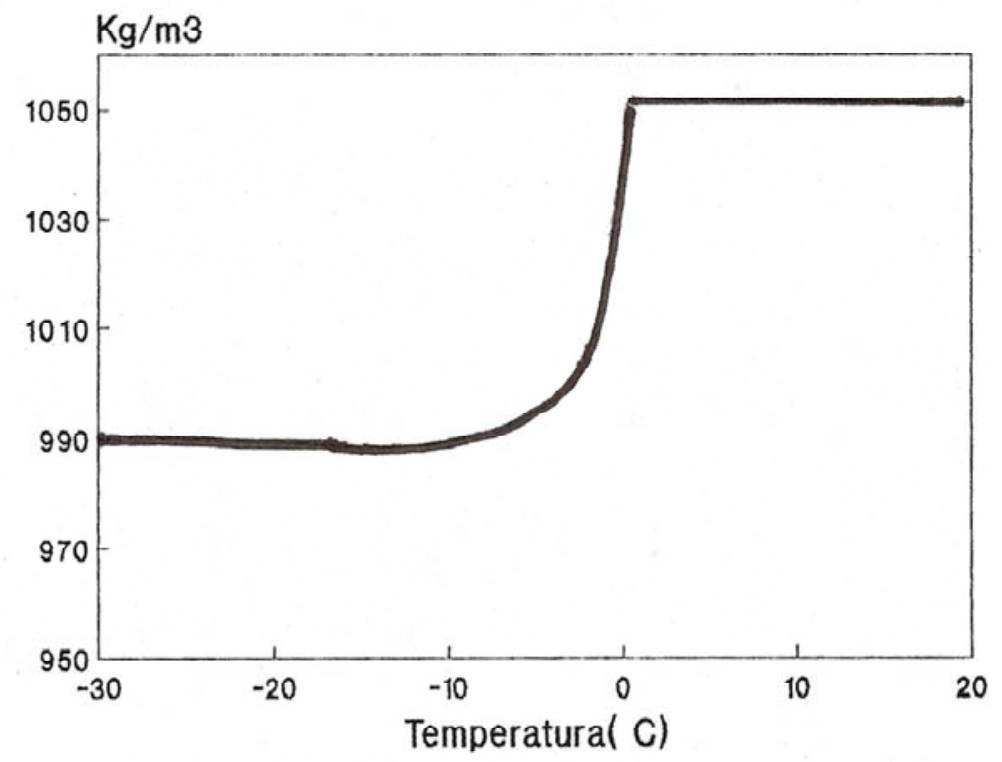

Fig. 1-4 Densidad del alimento en función de la temperatura en el rango de congelación 


\subsubsection{Resolución numérica de problemas de conducción de calor con cambio de fase}

Los problemas de conducción de calor con cambio de fase (llamados también tipo Stefan) que han sido resueltos numéricamente se pueden dividir en dos grupos, uno está basado en monitorear la frontera móvil de cambio de fase utilizando técnicas de localización de la posición de interfase a cada tiempo (Crank, 1984).

Este tipo de técnica es en general lenta y tiene complicaciones adicionales cuando existen geometrías multi-dimensionales (Crank, 1984; Comini y col., 1990). Los métodos con frontera móvil dan resultados precisos, no-oscilatorios, para la posición de frontera de hielo y temperatura de fusión dentro del producto, sin embargo la mayoría de los alimentos no presenta una única temperatura de cambio de fase sino que se congela gradualmente dentro de un rango específico, lo cual dificulta definir exactamente la posición de la frontera (Pham, 2006, 2009). El otro grupo de técnicas numéricas incorpora los efectos del cambio de fase en las propiedades termofísicas; densidad, conductividad térmica, y calor específico aparente, teniendo en cuenta su dependencia con la temperatura. En este caso se considera un calor específico aparente en un dominio con grilla fija. En la Fig. 1-5 se muestran dos tipos de curvas que representan el calor específico aparente en función de la temperatura; a) para un producto con cambio de fase abrupto y b) para un producto con un rango de temperatura de fusión.

En cada incremento del tiempo se debe estimar el nuevo valor de calor específico a través de la curva $\mathrm{Cp}_{\mathrm{ap}}$ versus temperatura. Sin embargo es muy difícil obtener una convergencia utilizando esta técnica porque siempre existe la posibilidad de estimar por defecto el pico que representa el calor latente.

Existen en la literatura varias técnicas propuestas para evitar este problema utilizando el MEF y calcular el calor específico efectivo cerca del rango de fusión (Cleland y col., 1984; Comini y col., 1989), sin embargo estas mejoras no conducen a resultados satisfactorios (Pham, 1995, 2008). 


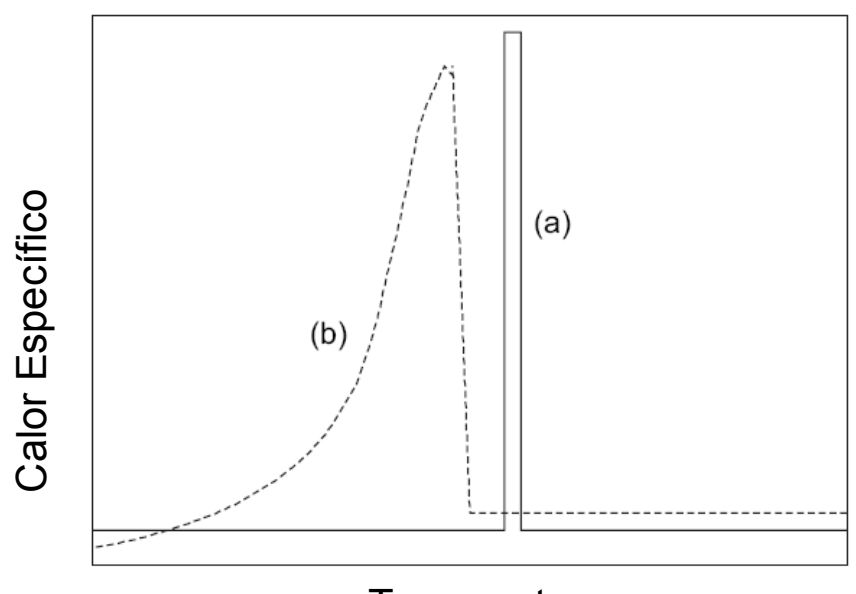

Temperatura

Fig. 1-5 Calor específico aparente para a) material con cambio de fase abrupto, b) material con cambio de fase gradual. (Gráfico adoptado de Pham, 2008)

Por estas razones las formulaciones entálpicas se desarrollaron con éxito y fueron utilizadas por varios autores (Mannapperuma y Singh, 1988 y 1989, Crank, 1984), donde la entalpía se la define como la integral del calor específico aparente $\left(H_{a}=\int \rho C p_{a p} d T\right)$. A su vez la integral de la conductividad con la temperatura representa la función de Kirchhoff $\left(E=\int k d T\right)$, que es una función más suave con la temperatura que la conductividad térmica versus $T$. Introduciendo simultáneamente ambas variables, $\mathrm{H}_{a}$ y $\mathrm{E}$, en la ecuación de conducción de calor se logra que el problema no-lineal se transforme en un problema lineal más simple de resolver. Esta metodología fue propuesta y utilizada por Fikiin $(1996,1998)$ y Scheerlinck y col. (2001) presentando diversas ventajas como la convergencia del método a pesar de los cambios abruptos en las propiedades termofísicas, y la reducción en los tiempos de ejecución de máquina (tiempos de CPU). Existen escasos trabajos en la literatura donde se aplica este método combinado de transformación de variables.

Los programas comerciales que utilizan el método de los elementos finitos para resolver problemas diversos de ecuaciones diferenciales parciales, presentan a veces dificultades cuando se necesita resolver problemas 
altamente no-lineales combinados con dominios irregulares; por ejemplo durante la simulación de procesos con cambio de fase (congelación y/o descongelación). Como se explicó anteriormente de acuerdo con el tipo de producto y su contenido de agua el calor específico aparente presenta a veces un pico cuasi delta de Dirac en el rango de fusión del alimento. El área debajo de la curva del $\mathrm{Cp}$ versus $\mathrm{T}$ en el rango de congelación representa el calor latente entregado debido a la fusión del alimento, por lo que se podrían suavizar las funciones termofísicas ensanchando la base del pico del calor específico. Si bien una forma de encarar el problema sería suavizando dichas funciones y manteniendo la misma área debajo de la curva, esto alteraría tanto el problema matemático planteado originalmente como la realidad física, ya que el cambio "real" de fase se produce en un rango más pequeño que el descrito por la función. Sin embargo en simuladores de aplicación general como COMSOL suavizar el Cp aparente es el único método disponible (Pham, 2008). En el caso del simulador comercial ANSYS donde se utiliza una formulación entálpica, sin la transformación de Kirchhoff, se logran mejores resultados en cuanto a la simulación de procesos con cambio de fase (Scheerlinck y col., 2001).

Para resolver un problema con cambio de fase utilizando la formulación entálpica y de Kirchhoff se requiere programar en un código computacional en un lenguaje de programación como puede ser Fortran o Matlab. Un código computacional escrito por el usuario (código abierto) es más sencillo de modificar y comprender cuando se busca acoplar subrutinas específicas, que son de interés para simular el problema. A su vez permite detectar dónde existen los problemas de convergencia numérica y cuales son los puntos que más afectan los tiempos de ejecución de máquina en función de generar posteriores mejoras y optimizaciones del código computacional.

A partir de esto se puede concluir que resulta necesario profundizar la aplicación del método de elementos finitos para resolver problemas de transferencia de energía en sistemas de geometría irregular a efectos de poder 
superar las limitaciones de los paquetes comerciales cuando se quieren resolver problemas de elevada complejidad especialmente con fuertes nolinealidades.

\subsection{Coeficiente de transferencia de calor en la interfase}

(h)

El coeficiente de transferencia de calor en la interfase $\left(\mathrm{h}\left[\mathrm{W} / \mathrm{m}^{2}{ }^{\circ} \mathrm{C}\right]\right)$ refleja las condiciones de transferencia sobre la superficie expuesta del alimento y el medio externo que lo rodea. Es muy importante para el cálculo de tiempos de congelación y en general presenta una variabilidad mayor que las propiedades termofísicas del producto, lo cual genera error en los cálculos de temperatura. En el proceso de calentamiento o enfriamiento la transferencia de calor depende de la velocidad del fluido externo, la geometría del alimento, las características como rugosidad de la superficie expuesta del alimento, además de otros factores. Todos estos factores en la práctica se consideran englobados dentro un solo factor, que se lo denomina coeficiente de transferencia (h) (Nesvadba, 2008), definido por $q=h\left(T_{s}-T_{\text {ext }}\right)$, donde $q$ es el calor por unidad de área y tiempo, $\mathrm{T}_{\mathrm{s}}$ es la temperatura en la superficie y $\mathrm{T}_{\text {ext }}$ es la temperatura del fluido externo.

Existen numerosas correlaciones para determinar el coeficiente de transferencia de calor en la interfase alimento-fluido que tienen en cuenta los números adimensionales Reynolds $\left(\operatorname{Re}=\frac{\rho v D}{\mu}\right)$ y Nusselt $\left(\mathrm{Nu}=\frac{\mathrm{hD}}{\mathrm{k}}\right)$ para distintas técnicas y equipos de congelación, tipo de flujos, etc. En convección natural se utilizan correlaciones de $\mathrm{Nu}=\mathrm{f}(\mathrm{Gr}, \mathrm{Pr})$, donde $\mathrm{Gr}$ es el número de Grashof $\left(G r=\frac{D^{3} \rho^{2} g \beta \Delta T}{\mu^{2}}\right)$ y $\operatorname{Pr}$ es el número de Prandtl $\left(\operatorname{Pr}=\frac{C p \mu}{k}\right)$.

Para convección forzada se utilizan correlaciones de la forma : $\mathrm{Nu}=\mathrm{f}(\mathrm{Re}, \mathrm{Pr})$. 
Es difícil determinar con exactitud el valor del coeficiente de transferencia sino se tienen datos de velocidad de fluido del aire o tipo de flujo (paralelo o transversal al objeto) por lo que se debe determinar para cada equipo en particular.

En el presente trabajo de Tesis se determinarán los coeficientes de transferencia calórica en la interfase para los sistemas bajo estudio, detallándose la metodología de obtención.

\subsection{Objetivos}

El objetivo general de la presente Tesis fue profundizar en los conceptos matemáticos del método de los elementos finitos con el fin de desarrollar programas computacionales propios (códigos abiertos) que permitan simular problemas de transferencia de calor con y sin cambio de fase en sistemas bi- y tridimensionales de geometría irregular validando experimentalmente dichos modelos.

En el caso de dominios irregulares la cantidad, disposición y numeración de los elementos utilizados para la discretización establecen una dificultad adicional para resolver el problema numérico, más aún en geometrías tridimensionales. Por lo tanto uno de los objetivos específicos fue desarrollar programas computacionales propios en elementos finitos para sistemas tridimensionales irregulares y las subrutinas particulares para incorporar la información de la malla en el programa principal.

Los sistemas alimenticios seleccionados para modelar surgieron a partir de necesidades planteadas por la industria y fueron descriptos en la Sección 1.2. Se planteó trabajar con tres sistemas de dificultades matemáticas crecientes: a) un producto cárneo (embutido) sometido a calentamiento en el que se buscaba una condición de inocuidad, b) un producto irregular heterogéneo con regiones de distintas propiedades termofísicas sometido a enfriamiento, c) congelación de un producto panificado de geometría irregular. 
Los objetivos específicos de la presente Tesis según el proceso de transferencia de energía y la geometría abordada en la simulación son:

A. Proceso de tratamiento térmico de embutidos (morcillas) en sistemas batch.

i. Desarrollar un código computacional propio que simule la transferencia de calor en sistemas de geometría bidimensional irregular con condición de borde convectivo.

ii. Implementar el código en el procesamiento térmico de elaboración de productos cárnicos de geometría bidimensional de revolución, acoplando además la cinética de destrucción de microorganismos patógenos.

iii. Incorporar al programa principal una subrutina de cálculo, tal como el balance macroscópico de energía, para la resolución de problemas de control de procesos a nivel industrial. Validar las predicciones numéricas mediante resultados experimentales de historias térmicas en equipos de calentamiento.

iv. Establecer las condiciones tiempo-temperatura necesarias para obtener un producto inocuo, teniendo en cuenta condiciones de proceso variable.

B. Proceso de refrigeración de productos semi-elaborados (empanadas):

i. Desarrollar un código computacional propio que simule la transferencia de calor en alimentos de geometría tridimensional irregular.

ii. Implementar el código computacional para simular la refrigeración de alimentos de geometría irregular y de composición heterogénea dada por la existencia de dominios con distintas propiedades termofísicas y validar experimentalmente las historias térmicas.

iii. Medición de las propiedades físicas como el calor específico de 
cada producto (fase cárnea y fase farinácea) utilizando Calorimetría Diferencial de Barrido (DSC).

\section{Proceso de congelación de productos semi-elaborados (croissant- medialunas):}

i. Desarrollar un código computacional propio que simule la transferencia de calor con cambio de fase en una geometría tridimensional irregular utilizando un método que combine la formulación entálpica y de Kirchhoff y compararla con las técnicas tradicionales que utilizan calor específico aparente.

ii. Implementar el programa para simular el problema no lineal de congelación de un alimento de geometría irregular que tenga en cuenta la variación de las propiedades termofísicas con la temperatura.

iii. Realizar la medición de las propiedades físicas como el calor específico aparente utilizando Calorimetría Diferencial de Barrido (DSC) y la determinación experimental de los coeficientes de transferencia calórica en la interfase en los sistemas estudiados.

iv. Validar las predicciones numéricas mediante resultados experimentales de historias térmicas en equipos de congelación a escala piloto.

Para cumplimentar los objetivos propuestos en el presente trabajo de Tesis se ha encarado por un lado el modelado matemático y la resolución numérica de procesos lineales donde las propiedades térmicas del alimento se consideraron constantes y por el otro, el análisis y modelado computacional de procesos con cambio de fase y propiedades termofísicas variables con la temperatura. 


\subsection{Esquema general de la Tesis}

En el Capítulo 2 de la presente Tesis se aborda la formulación matemática del método de los elementos finitos para dos dimensiones con propiedades constantes. Se describen los conceptos matemáticos y consideraciones generales para la elaboración del programa computacional. Luego se realizan comparaciones de resultados numéricos con datos de literatura para validar el modelo numérico.

En el Capítulo 3 se aplica el código computacional desarrollado en una geometría bidimensional irregular de revolución para simular el proceso de calentamiento de productos cárneos acoplando las cinéticas de inactivación de microorganismos patógenos. Al programa computacional escrito en un código abierto se le incorpora una subrutina que permite acoplar el balance macroscópico de energía. Se realiza la validación experimental a través de experiencias de laboratorio donde se compararan perfiles de temperatura con las predicciones numéricas.

En el Capítulo 4 se describen los conceptos matemáticos necesarios para generar un código computacional que describe la transferencia de calor en geometrías tridimensionales. Se realizan las validaciones correspondientes mediante soluciones analíticas y se comparan con datos de literatura de productos cárneos conteniendo tejidos muscular y graso, los cuales presentan distintas propiedades termofísicas.

En el Capítulo 5 se aplica el código generado en el Capítulo anterior para simular la refrigeración de un producto alimenticio de geometría tridimensional irregular (empanadas) constituido por dos materiales (masa y carne picada cocida). El programa se valida realizando experiencias de refrigeración de empanadas en una cámara.

En el Capítulo 6 se describe el procedimiento matemático para la obtención de un código computacional que describe el proceso de congelación de un producto farináceo de geometría irregular. Se utiliza un código propio para procesos con cambio de fase implementando la formulación entálpica y de 
Kirchhoff en una geometría tridimensional. El programa se valida realizando experiencias de congelación del producto en un túnel a escala piloto.

En el Capítulo 7 se describen las conclusiones referentes al desarrollo del programas computacionales propios utilizados para simular sistemas irregulares bi y tridimensionales, además de los procesos de transferencia de energía modelados. 
Capítulo 2 


\section{Resolución de problemas de transferencia de calor mediante MEF en dos dimensiones}

En el presente Capítulo se aborda la resolución del balance microscópico diferencial de energía mediante el método de los elementos finitos para el caso de una geometría irregular bidimensional.

Se considera el caso con propiedades termofísicas (conductividad térmica, calor específico, y densidad) independientes de la temperatura y de la posición dentro del dominio de estudio. Este tipo de problemas constituyen matemáticamente un problema lineal.

\subsection{Problemas lineales en dos dimensiones (2D)}

La ecuación general que representa la transferencia de energía en estado transiente sin reacción química en dos dimensiones es la siguiente:

$$
\rho \operatorname{Cp} \frac{\partial T}{\partial t}=k\left(\frac{\partial^{2} T}{\partial x^{2}}+\frac{\partial^{2} T}{\partial y^{2}}\right) \quad \text { en } \Omega
$$

Esta ecuación es válida en el dominio $\Omega$ siendo T la temperatura, t el tiempo de proceso, $\rho$ la densidad, $\mathrm{k}$ la conductividad térmica y $\mathrm{Cp}$ el calor específico del material, $\mathrm{x}$ e y las coordenadas geométricas.

Condición inicial:

$\mathrm{T}=\mathrm{T}_{0} \quad \mathrm{t}=0 \quad$ en $\Omega$

Se analizaron condiciones de contorno de temperatura prescripta y con transferencia convectiva: 


$$
\mathrm{T}=\mathrm{T}_{1} \quad \mathrm{t} \geq 0 \quad \text { en } \delta \Omega_{2}
$$

$$
-k\left(\frac{\partial T}{\partial x} \cdot n_{x}+\frac{\partial T}{\partial y} \cdot n_{y}\right)=h\left(T-T_{\text {ext }}\right) \quad t \geq 0 \quad \text { en } \delta \Omega_{1}
$$

donde $\delta \Omega_{1}$ y $\delta \Omega_{2}$ corresponden a la interfase convectiva y a la interfase donde la temperatura está prescripta, respectivamente, $T_{\text {ext }}$ es la temperatura del medio calefactor, $T_{0}$ la temperatura inicial, $n=\left(n_{x}, n_{y}\right)$ es el vector normal unitario exterior a la superficie, y $\mathrm{h}$ el coeficiente de transferencia de calor. A modo de ejemplo se muestra la siguiente Figura 2-1:

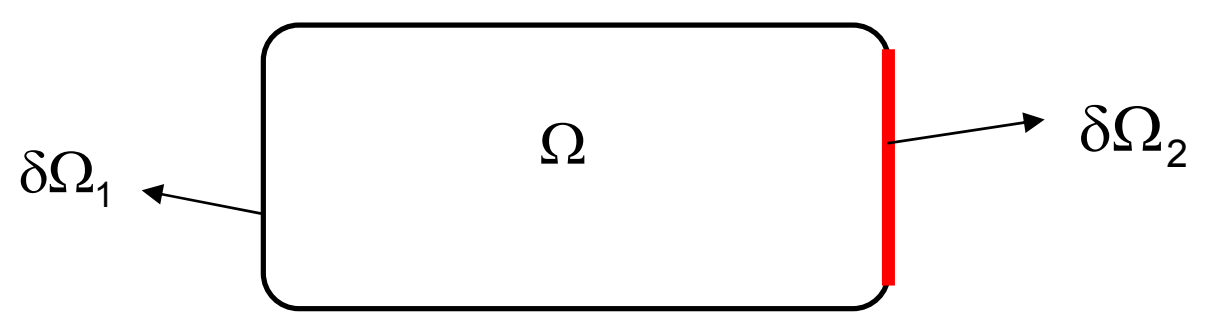

Fig. 2-1 Dominio de validez de la ecuación diferencial e interfases involucradas

Cabe aclarar que $\Omega$ en este caso corresponde a una superficie y $\delta \Omega$ a una línea que representa algún borde del dominio, pudiendo extenderse la misma notación para tres dimensiones donde intervienen volúmenes y superficies. De esta forma queda planteado el problema matemático con derivadas parciales representado por el balance microscópico diferencial de energía. En primer lugar para construir la formulación variacional del problema es necesario definir el residuo $\varepsilon$ como:

$$
\varepsilon(x, y)=\rho \operatorname{Cp} \frac{\partial T}{\partial t}-k\left(\frac{\partial^{2} T}{\partial x^{2}}+\frac{\partial^{2} T}{\partial y^{2}}\right)
$$

El residuo se construye con la aproximación de $\mathrm{T}$ de tal forma que $\varepsilon$ sea 
el menor posible. Luego se multiplica el residuo por una función de prueba v, la cual debe cumplir que el producto $\varepsilon v$ sea suave y su integral en todo el dominio sea cero. Por ende se obtiene la siguiente ecuación:

$$
\int_{\Omega} v \cdot \varepsilon(x, y) d \Omega=\int_{\Omega} v \cdot \rho C p \frac{\partial T}{\partial t} d \Omega-\int_{\Omega} v \cdot k\left(\frac{\partial^{2} T}{\partial x^{2}}+\frac{\partial^{2} T}{\partial y^{2}}\right) d \Omega=0
$$

Por medio de la regla de diferenciación resulta:

$\nabla \cdot(\mathrm{vk} \nabla \mathrm{T})=\mathrm{k} \nabla \mathrm{T} \cdot \nabla \mathrm{v}+\mathrm{v} \nabla \cdot(\mathrm{k} \nabla \mathrm{T})$

donde $\nabla \mathrm{T}$ es un vector:

$$
\nabla \mathrm{T}=\left(\frac{\partial \mathrm{T}}{\partial \mathbf{x}}, \frac{\partial \mathrm{T}}{\partial \mathrm{y}}\right)
$$

Si se considera k isotrópico en todo el dominio e independiente de $\mathrm{T}$ se obtiene: $\mathrm{v} \nabla \cdot(\mathrm{k} \nabla \mathrm{T})=\mathrm{vk}\left(\nabla^{2} \mathrm{~T}\right)$. Por lo tanto se puede reemplazar el segundo término utilizando la siguiente ecuación:

$\mathrm{vk}\left(\nabla^{2} \mathrm{~T}\right)=\nabla \cdot(\mathrm{vk} \nabla \mathrm{T})-\mathrm{k} \nabla \mathrm{T} \cdot \nabla \mathrm{v}$

donde

$$
\nabla^{2} \mathbf{T}=\frac{\partial^{2} \mathrm{~T}}{\partial \mathbf{x}^{2}}+\frac{\partial^{2} \mathrm{~T}}{\partial \mathbf{y}^{2}}
$$

resultando en la ecuación 2.6: 


$$
\int_{\Omega} \mathrm{v} \cdot \rho \operatorname{Cp} \frac{\partial \mathrm{T}}{\partial \mathrm{t}} \mathrm{d} \Omega-\int_{\Omega} \nabla \cdot(\mathrm{vk} \nabla \mathrm{T}) \mathrm{d} \Omega+\int_{\Omega} \mathrm{k} \nabla \mathrm{T} \cdot \nabla \mathrm{v} \mathrm{d} \Omega=0
$$

El segundo término de la ecuación se puede transformar en una integral de borde mediante el teorema de la divergencia (Ec. 2.12):

$$
\int_{\Omega} \nabla \cdot \sigma \mathrm{d} \Omega=\int_{\delta \Omega} \sigma \cdot \mathrm{nd} \delta \Omega
$$

Siendo $\sigma$ en este caso vk $\nabla \mathrm{T}$, la Ec. 2.11 se expresa como:

$$
\int_{\Omega} \mathrm{v} \rho \operatorname{Cp} \frac{\partial \mathrm{T}}{\partial \mathrm{t}} \mathrm{d} \Omega-\int_{\delta \Omega} \mathrm{vk} \nabla \mathrm{T} \cdot \mathrm{nd} \delta \Omega+\int_{\Omega} \mathrm{k} \nabla \mathrm{T} \cdot \nabla \mathrm{vd} \Omega=0
$$

Sustituyendo las condiciones de borde naturales en la integral de borde $\delta \Omega:$

$$
\int_{\Omega} \mathrm{v} \cdot \rho \operatorname{Cp} \frac{\partial \mathrm{T}}{\partial \mathrm{t}} \mathrm{d} \Omega+\int_{\delta \Omega_{1}} \mathrm{vh}\left(\mathrm{T}-\mathrm{T}_{\text {ext }}\right) \mathrm{d} \delta \Omega_{1}+\int_{\Omega} \mathrm{k} \nabla \mathrm{T} \cdot \nabla \mathrm{v} \mathrm{d} \Omega=0
$$

Se puede observar que las condiciones de borde esenciales (temperatura prescripta) no intervienen en la formulación variacional, debiendo integrar solamente sobre el borde $\delta \Omega_{1}$, ya que establece una condición de flujo en la interfase (o sea una derivada de la temperatura).

Por lo tanto el problema planteado corresponde a encontrar una función $\mathrm{T}$ que sea $\mathrm{T}_{1}$ en $\delta \Omega_{2}$ y que cumpla para cualquier función de prueba $\mathrm{v}$ admisible:

$$
\int_{\Omega} v \cdot \rho \operatorname{Cp} \frac{\partial \mathrm{T}}{\partial \mathrm{t}} \mathrm{d} \Omega+\int_{\delta \Omega_{1}} \mathrm{vhTd} \delta \Omega_{1}+\int_{\Omega} \mathrm{k} \nabla \mathrm{T} \cdot \nabla \mathrm{vd} \Omega=\int_{\delta \Omega_{1}} \mathrm{vhT} \mathrm{T}_{\mathrm{ext}} \mathrm{d} \delta \Omega_{1}
$$




\section{(F)}

C I D C A

Para encontrar las funciones $v$ que sean apropiadas para resolver el problema vemos que se requiere que la integral del producto de las derivadas parciales de $\mathrm{T}$ y v esté definida. Por lo tanto es suficiente utilizar una clase de funciones denominadas $\mathrm{H}^{1}$ cuyas derivadas parciales de orden uno e inferior sean de cuadrado integrable en $\Omega$ (se debe contemplar la posibilidad de que $\mathrm{v}$ y $\mathrm{T}$ sean iguales). En otras palabras una función $v$ pertenece a $\mathrm{H}^{1}$ si :

$\int_{\Omega}\left[\left(\frac{\partial v}{\partial x}\right)^{2}+\left(\frac{\partial v}{\partial y}\right)^{2}+v^{2}\right] d \Omega<\infty$

Además las funciones $v$ utilizadas deben cumplir con las condiciones esenciales donde $T=T_{1}$ por lo que deben valer cero $(v=0)$ en $\delta \Omega_{2}$.

La ecuación 2.15 representa la formulación variacional de la ecuación diferencial correspondiente al balance microscópico de energía y tiene las siguientes ventajas: 1) si se encuentra una solución de la formulación variacional ésta satisface el balance microscópico de energía y viceversa, una solución de la ecuación 2.15 es una solución de la ecuación 2.1-2.4. 2) la formulación variacional del problema presenta restricciones más débiles (derivadas parciales de cuadrado integrable) que las que exige el problema 2.12.4 (derivadas parciales de segundo orden). 3) a partir de la formulación variacional se puede construir la solución aproximada utilizando el método de los elementos finitos. El método consiste en encontrar una solución aproximada a la Ec. 2.15 (formulación variacional) en un subespacio de dimensión finita del espacio de soluciones admisibles $\mathrm{H}^{1}$. 


\subsection{Discretización del dominio}

El objetivo es representar a las funciones $T$ y $v$ mediante una aproximación numérica $T_{h}$ y $v_{h}$ utilizando polinomios definidos a trozos sobre el dominio $\Omega_{\mathrm{h}}$. La discretización del dominio $\Omega$ en $\Omega_{\mathrm{h}}$ debe hacerse con un tipo de elementos que mantenga lo mejor posible la figura irregular del dominio inicial y a su vez sean lo suficientemente simples en virtud de minimizar los esfuerzos computacionales. En el caso del plano los elementos triangulares cumplen adecuadamente con los propósitos anteriores.

Una vez que se ha construido la malla en pequeños elementos triangulares en cuyos vértices se ubican a los nodos, se procede a generar los polinomios de interpolación o de base, los cuales estarán definidos a trozos en cada elemento de la malla. La solución aproximada $T_{h}$ es una función contínua en $\Omega_{\mathrm{h}}$ tal que en cada elemento es un polinomio construido en base a las funciones de interpolación, coincidiendo con la solución $T$ en los nodos. En particular $\mathrm{T}_{\mathrm{h}}$ es un elemento en $\mathrm{H}^{1}$.

La solución en cada elemento "e" formado por tres nodos es la siguiente:

$T_{h}{ }^{e}=T 1 \cdot h 1+T 2 \cdot h 2+T 3 \cdot h 3$

donde $\mathrm{Ti}$, con $\mathrm{i}=1,2,3$ son los coeficientes a determinar siendo las funciones base las siguientes:

$\mathrm{h} 1=\mathrm{r}$

$0 \leq r \leq 1$

$\mathrm{h} 2=\mathrm{s}$

$0 \leq \mathrm{s} \leq 1$

$h 3=1-r-s$ 
Una figura ilustrativa del triángulo de referencia se encuentra a continuación en la Figura 2-2.

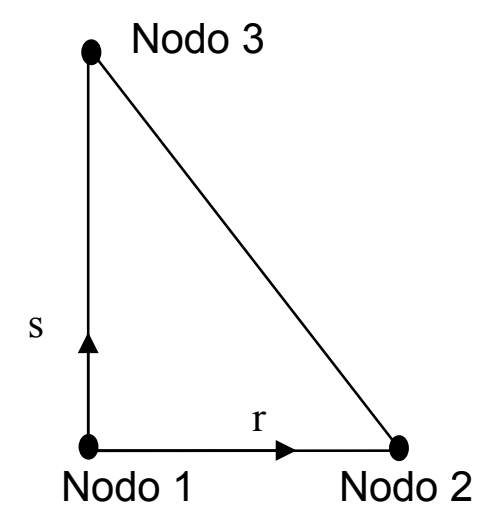

Fig. 2-2 Triángulo de referencia

Como puede observarse de la ecuación 2.17 cuando se evalúa un nodo "i" la función de base hi=1 y las demás son cero.

Utilizando el método Galerkin donde las funciones base o de interpolación se utilizan para representar tanto la solución $\mathrm{T}_{\mathrm{h}}$ como las funciones de prueba $v_{h}$ se define la función de prueba $v_{h}$ en el elemento "e":

$\mathrm{v}_{\mathrm{h}}{ }^{\mathrm{e}}=\alpha 1 \cdot \mathrm{h} 1+\alpha 2 \cdot \mathrm{h} 2+\alpha 3 \cdot \mathrm{h} 3$

Como los $\alpha \mathrm{i}$ con $\mathrm{i}=1,2,3$ son arbitrarios, se toma $\alpha \mathrm{i}=1$ y $\alpha \mathrm{j}=0$ para todo $i \neq j$ con el objetivo de obtener un sistema de ecuaciones cuyas incógnitas sean las Ti.

Reemplazando $v_{h}$ y $T_{h}$ y utilizando una notación matricial para simplificar la visualización se define:

$$
H=\left[\begin{array}{lll}
h 1 & h 2 & h 3
\end{array}\right] \quad H^{t}=\left[\begin{array}{l}
h 1 \\
h 2 \\
h 3
\end{array}\right]
$$


$T=\left[\begin{array}{lll}T 1 & T 2 & T 3\end{array}\right] \quad T^{t}=\left[\begin{array}{l}h 1 \\ h 2 \\ h 3\end{array}\right]$

$B=\left[\begin{array}{lll}h 1_{x} & h 2_{x} & h 3_{x} \\ h 1_{y} & h 2_{y} & h 3_{y}\end{array}\right]$ donde $h i_{x}=\frac{\partial h i}{\partial x}$ y hi ${ }_{y}=\frac{\partial h i}{\partial y}$ para $i=1,2,3$

Dado que $\mathrm{T} y \mathrm{v}$ dependen de $\mathrm{x}, \mathrm{y}$, siendo éstas a su vez dependientes de $r$, s se define la siguiente matriz $\mathrm{Hr}$ (la cual será posteriormente utilizada para el cálculo de las matrices elementales):

$H r=\left[\begin{array}{lll}h 1_{r} & h 2_{r} & h 3_{r} \\ h 1_{s} & h 2_{s} & h 3_{s}\end{array}\right]$ donde $h i_{r}=\frac{\partial h i}{\partial r}$ y hi ${ }_{s}=\frac{\partial h i}{\partial s}$ para $i=1,2,3$

Por lo tanto la temperatura en un elemento "e" se define como:

$\mathrm{T}_{\mathrm{h}}^{\mathrm{e}}=\mathrm{H} \cdot\left[\begin{array}{lll}\mathrm{T} 1 & \mathrm{~T} 2 & \mathrm{~T} 3\end{array}\right]^{\mathrm{t}}$

La derivada temporal en un elemento "e" se define como:

$\dot{\mathrm{T}}=\frac{\partial \mathrm{T}_{\mathrm{h}}{ }^{\mathrm{e}}}{\partial \mathrm{t}}=\mathrm{H} \cdot\left[\begin{array}{lll}\dot{\mathrm{T}} 1 & \dot{\mathrm{T}} 2 & \dot{\mathrm{T}} 3\end{array}\right]^{\mathrm{t}}$

Reemplazando en la ecuación 2.15 se obtiene (Zienkiewicz y Taylor, 1994a, 1994b):

$\left[\sum_{\mathrm{e}} \int_{\Omega_{\mathrm{e}}} \mathrm{H}^{\mathrm{t}} \rho \mathrm{CpHd} \Omega\right] \dot{\mathrm{T}}+\left\{\sum_{\mathrm{e} 1} \int_{\delta \Omega_{\mathrm{e} 1}} \mathrm{H}^{\mathrm{t}} \mathrm{hH} \mathrm{d} \delta \Omega_{\mathrm{e} 1}+\sum_{\mathrm{e}} \int_{\Omega_{\mathrm{e}}} \mathrm{B}^{\mathrm{t}} \mathrm{kBd} \Omega\right\} T=\left[\sum_{\mathrm{e} 1} \int_{\delta \Omega_{\mathrm{e} 1}} \mathrm{H}^{\mathrm{t}} \mathrm{h} \mathrm{T}_{\mathrm{ext}} \mathrm{d} \delta \Omega_{\mathrm{e} 1}\right] 2.28$

donde 


\section{(B)}

C I D C A

$\mathrm{CG}=\sum_{\mathrm{e}} \int_{\Omega_{\mathrm{e}}} \mathrm{H}^{\mathrm{t} \rho} \mathrm{CpH} \mathrm{d} \Omega \quad$ es la matriz global de capacitancia

$\mathrm{KG}=\sum_{\mathrm{e} 1} \int_{\delta \Omega_{\mathrm{e} 1}} H^{\mathrm{t}} h H \mathrm{~d} \delta \Omega_{\mathrm{e} 1}+\sum_{\mathrm{e}} \int_{\Omega_{\mathrm{e}}} \mathrm{B}^{\mathrm{t}} \mathrm{kB} \mathrm{d} \Omega \quad$ es la matriz global de conductancia

$F G=\sum_{\mathrm{e} 1} \int_{\delta \Omega_{\mathrm{e} 1}} H^{\mathrm{t} h} T_{\mathrm{ext}} \mathrm{d} \delta \Omega_{\mathrm{e} 1} \quad$ es el vector de fuerzas globales

El símbolo $\sum_{\mathrm{e}}$ en cada una de las matrices globales $\mathrm{CG}, \mathrm{KG}$ y el vector FG representa el cálculo de las integrales en cada elemento de la malla y su borde, según corresponda, para luego ser ensamblado en una matriz global según la numeración establecida de los nodos y elementos de la malla. Más adelante se hará referencia al ensamble (Sección 2.4). Por lo tanto se definen como matrices elementales a las matrices obtenidas a través del cálculo de un elemento cualquiera de la malla. Dado que un elemento está compuesto por tres nodos, las matrices elementales son de dimensión $3 \times 3$ y el vector de fuerzas es de $3 \times 1$. A modo de ejemplo, tomando la matriz elemental de capacitancia, donde interviene el producto de los vectores $\mathrm{H}^{t}$ (de dimensión $3 \times 1$ ) por $\mathrm{H}$ (dimensión $1 \times 3$ ) se obtiene una matriz elemental de $3 \times 3$, por ende la expresión $\mathrm{CG}^{\mathrm{e}}=\int_{\Omega_{\mathrm{e}}} \mathrm{H}^{\mathrm{t} \rho} \mathrm{CpH} \mathrm{d} \Omega$ representa matemáticamente

$\mathrm{CG}_{\mathrm{ij}}^{\mathrm{e}}=\int_{\mathrm{e}} \mathrm{h}_{\mathrm{i}} \rho \mathrm{Cph} \mathrm{h}_{\mathrm{j}} \mathrm{d} \Omega_{\mathrm{e}} \quad$ para $\mathrm{i}=1,2,3$ y $\mathrm{j}=1,2,3$

De manera equivalente se puede expresar en forma matricial según: 


\section{(F)}

C I D C A

$\mathrm{CG}^{\mathrm{e}}=\rho \cdot \mathrm{Cp} \cdot\left[\begin{array}{lll}\int \mathrm{h} 1 \cdot \mathrm{h} 1 \cdot \mathrm{d} \Omega_{\mathrm{e}} & \int \mathrm{h} 1 \cdot \mathrm{h} 2 \cdot \mathrm{d} \Omega_{\mathrm{e}} & \int \mathrm{h} 1 \cdot \mathrm{h} 3 \cdot \mathrm{d} \Omega_{\mathrm{e}} \\ \int \mathrm{h} 2 \cdot \mathrm{h} 1 \cdot \mathrm{d} \Omega_{\mathrm{e}} & \int \mathrm{h} 2 \cdot \mathrm{h} 2 \cdot \mathrm{d} \Omega_{\mathrm{e}} & \int \mathrm{h} 2 \cdot \mathrm{h} 3 \cdot \mathrm{d} \Omega_{\mathrm{e}} \\ \int \mathrm{h} 3 \cdot \mathrm{h} 1 \cdot \mathrm{d} \Omega_{\mathrm{e}} & \int \mathrm{h} 3 \cdot \mathrm{h} 2 \cdot \mathrm{d} \Omega_{\mathrm{e}} & \int \mathrm{h} 3 \cdot \mathrm{h} 3 \cdot \mathrm{d} \Omega_{\mathrm{e}}\end{array}\right]$

Luego del cálculo de cada elemento de la matriz $\mathrm{CG}^{\mathrm{e}}$, se produce el ensamble de la matriz elemental en la matriz global. Este procedimiento se explicará en la Sección 2.4.

El sistema semi-discreto de ecuaciones diferenciales ordinarias a resolver, descripto por la ecuación 2.28 , se puede escribir de manera más compacta y simple en forma matricial:

$\mathrm{CG} \cdot \dot{\mathrm{T}}+\mathrm{K} \cdot \mathrm{GT}=\mathrm{FG}$

Este sistema tiene la característica de ser un sistema de ecuaciones diferenciales ordinarias rígido, esto quiere decir que la matriz del sistema tiene autovalores grandes en módulo que obligaría a tomar pasos en el tiempo extremadamente pequeños para un esquema de resolución de integración en el tiempo de tipo explícito. La resolución del sistema de ecuaciones diferenciales ordinarias establecido en la ecuación 2.31 se describirá más detalladamente en la Sección 2.3.

Para visualizar el cálculo de cada una de las matrices y vectores elementales se utilizará un elemento cualquiera $\Omega_{\mathrm{e}}$ aplicando la transformación de variables a coordenadas locales utilizando el triángulo de referencia. La razón fundamental por la cual se requiere de una transformación de un elemento arbitrario $\Omega_{\mathrm{e}}$ en uno de referencia, es que los límites de integración en los cálculos de las matrices y vectores elementales cambiarían elemento a elemento y sería dificultoso aplicar una forma general y sistemática de calcular estas matrices. 

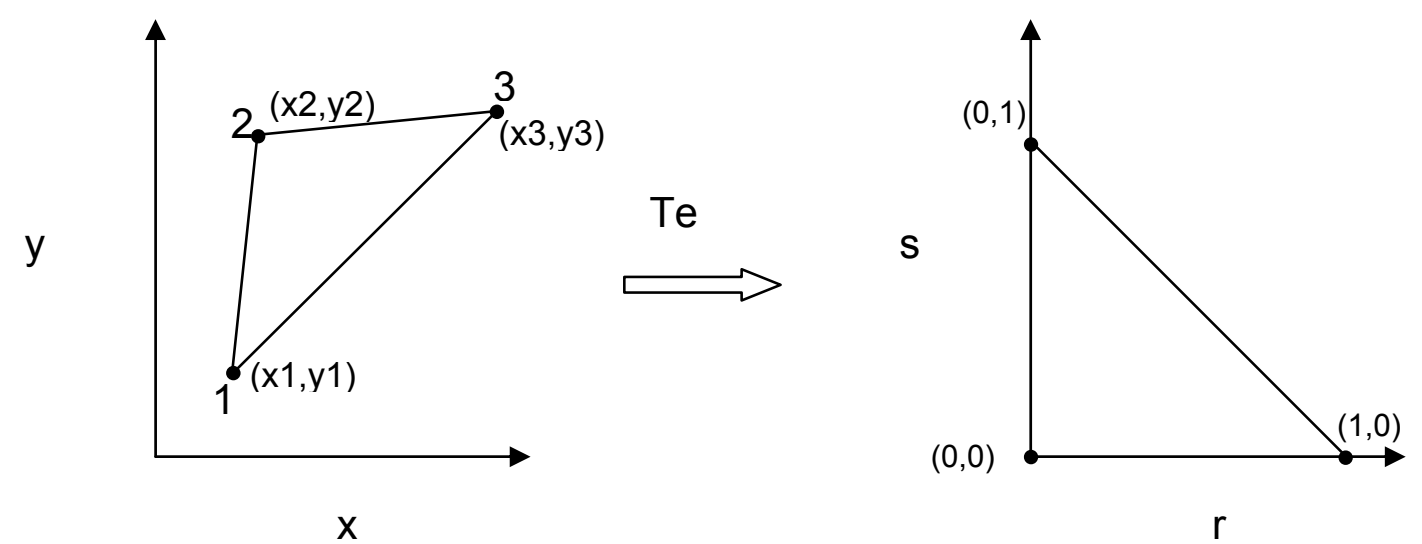

Fig. 2-3 Transformación de coordenadas del elemento triangular en el triángulo de referencia

Se define una transformación Te : $x=x(r, s), y=y(r, s)$ donde

$\mathrm{x}=\mathrm{x} 1 \cdot \mathrm{h} 1+\mathrm{x} 2 \cdot \mathrm{h} 2+\mathrm{x} 3 \cdot \mathrm{h} 3$

$y=y 1 \cdot h 1+y 2 \cdot h 2+y 3 \cdot h 3$

donde xi e yi con $i=1,2$, 3 son las coordenadas de los nodos locales y hi con $i=1,2,3$ son las funciones base definidas en las ecuaciones 2.18-2.20.

Si las funciones $x$ e y son diferenciales y continuas con respecto a $r$ y $s$ se define a los diferenciales

$$
\begin{aligned}
& d x=\frac{\partial x}{\partial r} d r+\frac{\partial x}{\partial s} d s \\
& d y=\frac{\partial y}{\partial r} d r+\frac{\partial y}{\partial s} d s
\end{aligned}
$$

Escritas en forma matricial 2.34 y 2.35 resulta: 


$$
\left[\begin{array}{l}
d x \\
d y
\end{array}\right]=\left[\begin{array}{ll}
\partial x / \partial r & \partial x / \partial s \\
\partial y & \partial y / \partial s
\end{array}\right] \cdot\left[\begin{array}{l}
d r \\
d s
\end{array}\right]
$$

La matriz de 2x2 es la matriz jacobiana de la transformación y se denota como J. El determinante $\operatorname{det}(\mathrm{J})$ se define como

$$
|J|=\operatorname{det}(J)=\frac{\partial x}{\partial r} \frac{\partial y}{\partial s}-\frac{\partial x}{\partial s} \frac{\partial y}{\partial r}
$$

Por ende si $|\mathrm{J}| \neq 0$ se puede deducir que:

$$
\left[\begin{array}{c}
d r \\
d s
\end{array}\right]=J^{-1} \cdot\left[\begin{array}{l}
d x \\
d y
\end{array}\right]=\frac{1}{|J|}\left[\begin{array}{cc}
\partial y / \partial s & -\partial x / \partial s \\
-\partial y / \partial r & \partial x / \partial r
\end{array}\right] \cdot\left[\begin{array}{l}
d x \\
d y
\end{array}\right]
$$

la cual representa la transformación inversa de Te; es decir $\mathrm{Te}^{-1}: r=r(x, y)$ $\mathrm{s}=\mathrm{s}(\mathrm{x}, \mathrm{y})$ por lo que análogamente se puede escribir

$$
\left[\begin{array}{l}
d r \\
d s
\end{array}\right]=\left[\begin{array}{cc}
\partial r / \partial x & \partial r \\
\partial s & \partial s \\
\partial x & \partial y
\end{array}\right] \cdot\left[\begin{array}{l}
d x \\
d y
\end{array}\right]
$$

Igualando 2.38 a 2.39

$$
\left[\begin{array}{cc}
\partial r / \partial \mathbf{x} & \partial \mathrm{r} / \partial \mathbf{y} \\
\partial \mathbf{s} / \partial \mathbf{x} & \partial \mathbf{s} / \partial \mathbf{y}
\end{array}\right]=\frac{1}{|\mathbf{J}|}\left[\begin{array}{cc}
\partial \mathbf{y} / \partial \mathbf{s} & -\partial \mathbf{x} / \partial \mathbf{s} \\
-\partial \mathbf{y} / \partial \mathbf{r} & \partial \mathbf{x} / \partial \mathbf{r}
\end{array}\right]
$$

Estas relaciones resultan importantes para el cálculo de la matriz por elemento ya que en ella interviene la matriz $B$, según la ecuación 2.28 , cada 


\section{(F)}

C I D C A

$h i_{x}=\frac{\partial h i}{\partial x} y h_{y}=\frac{\partial h i}{\partial y}$, las cuales se representan como:

$\frac{\partial h i}{\partial x}=\frac{\partial h i}{\partial r} \frac{\partial r}{\partial x}+\frac{\partial h i}{\partial s} \frac{\partial s}{\partial x}=\frac{\partial h i}{\partial r}\left(\frac{1}{|J|} \frac{\partial y}{\partial s}\right)+\frac{\partial h i}{\partial s}\left(-\frac{1}{|J|} \frac{\partial y}{\partial r}\right) \quad$ para i=1, 2, 3

$\frac{\partial h i}{\partial y}=\frac{\partial h i}{\partial r} \frac{\partial r}{\partial y}+\frac{\partial h i}{\partial s} \frac{\partial s}{\partial y}=\frac{\partial h i}{\partial r}\left(-\frac{1}{\mid J} \frac{\partial x}{\partial s}\right)+\frac{\partial h i}{\partial s}\left(\frac{1}{|J|} \frac{\partial x}{\partial r}\right) \quad$ para $i=1,2,3$

Las funciones base de interpolación (hi) son lineales por lo tanto sus derivadas $\mathrm{hi}_{\mathrm{r}}=\frac{\partial \mathrm{hi}}{\partial \mathrm{r}}$ y $\mathrm{hi}_{\mathrm{s}}=\frac{\partial \mathrm{hi}}{\partial \mathrm{s}}$ serán constantes. En el caso de elementos triangulares lineales resulta:

$\mathrm{Hr}=\left[\begin{array}{lll}\mathrm{h} 1_{\mathrm{r}} & \mathrm{h} 2_{\mathrm{r}} & \mathrm{h} 3_{\mathrm{r}} \\ \mathrm{h} 1_{\mathrm{s}} & \mathrm{h} 2_{\mathrm{s}} & \mathrm{h} 3_{\mathrm{s}}\end{array}\right]=\left[\begin{array}{ccc}1 & 0 & -1 \\ 0 & 1 & -1\end{array}\right]$

En cada elemento, según cuales sean las coordenadas de sus nodos resulta:

$\frac{\partial x}{\partial r}=x 1 \cdot h 1 r+x 2 \cdot h 2 r+x 3 \cdot h 3 r$

$\frac{\partial x}{\partial s}=x 1 \cdot h 1 s+x 2 \cdot h 2 s+x 3 \cdot h 3 s$

$\frac{\partial y}{\partial r}=y 1 \cdot h 1 r+y 2 \cdot h 2 r+y 3 \cdot h 3 r$ 


$$
\frac{\partial y}{\partial r}=y 1 \cdot h 1 r+y 2 \cdot h 2 r+y 3 \cdot h 3 r
$$

Reemplazando las ecuaciones 2.44 a 2.47 en 2.41 y 2.42 se construye la matriz $B$ en la matriz del elemento.

En el cálculo de las matrices y vectores de los elementos, una vez seleccionada la transformación de acuerdo a las funciones de forma elegidas, intervienen integrales de polinomios en las variables $r$ y s. Para evaluar dichas integrales numéricamente, se utilizan reglas de cuadratura cuyo orden se puede elegir lo suficientemente alto como para que el valor de la integral sea exacto.

La fórmula de integración numérica utilizando el método de Gauss para triángulos es la siguiente:

$$
\iint \mathrm{Fdrds}=\frac{1}{2} \sum \mathrm{w}_{\mathrm{i}} \mathrm{F}\left(\mathrm{r}_{\mathrm{i}}, \mathrm{s}_{\mathrm{i}}\right)
$$

Siendo $\mathrm{F}$ en este caso una función polinómica en $r$ y $s, y w_{i}$ los pesos correspondientes a cada $\left(\mathrm{r}_{\mathrm{i}}, \mathrm{s}_{\mathrm{i}}\right)$ utilizado.

En la Tabla 2-1 se pueden observar los distintos puntos de integración que deben elegirse según el grado de precisión deseado para el cálculo. El grado de precisión se define como el grado máximo del polinomio que se integra exactamente.

\subsection{Discretización temporal (Método a)}

La ecuación 2.31 requiere para su completa discretización una estimación de la variable $\dot{\mathrm{T}}=\frac{\partial \mathrm{T}}{\partial \mathrm{t}}$ en cada uno de los nodos. 

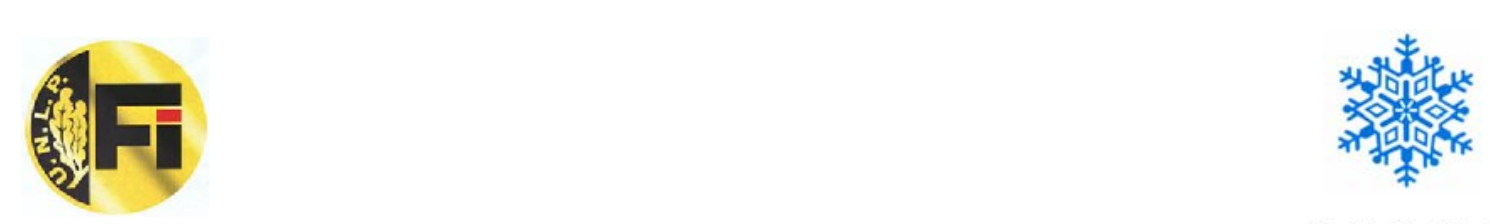

C I D C A

Tabla 2-1 Puntos de integración numérica para triángulos utilizando método Gauss (Adaptada de Bathe, 1996)

\begin{tabular}{|c|c|c|c|c|c|}
\hline $\begin{array}{l}\text { Orden de } \\
\text { integración }\end{array}$ & $\begin{array}{l}\text { Grado de } \\
\text { precisión }\end{array}$ & $\begin{array}{l}\text { Puntos de } \\
\text { Integración }\end{array}$ & $\begin{array}{c}\text { Coordenada } \\
\mathbf{r}\end{array}$ & $\begin{array}{c}\text { Coordenada } \\
5\end{array}$ & Pesos \\
\hline 3 puntos & 2 & & $\begin{array}{l}r_{1}=0.1666666666667 \\
r_{2}=0.6666666666667 \\
r_{3}=r_{1}\end{array}$ & $\begin{array}{l}s_{1}=r_{1} \\
s_{2}=r_{1} \\
s_{3}=r_{2}\end{array}$ & $\begin{array}{l}w_{1}=0.3333333333333 \\
w_{2}=w_{1} \\
w_{3}=w_{1}\end{array}$ \\
\hline 7 puntos & 5 & & $\begin{array}{l}r_{1}=0.1012865073235 \\
r_{2}=0.7974269853531 \\
h_{2}=r_{1} \\
r_{4}=0.4701420641051 \\
r_{5}=r_{4}= \\
r_{6}=0.0597158717898 \\
r_{7}=0.3333333333333\end{array}$ & $\begin{array}{l}s_{1}=r_{1} \\
s_{2}=r_{1} \\
s_{1}=r_{2} \\
s_{4}=r_{6} \\
S_{5}=r_{4} \\
s_{4}=r_{4} \\
s_{7}=r_{7}\end{array}$ & $\begin{array}{l}w_{1}=0.1259391805448 \\
w_{2}=w_{1} \\
w_{3}=w_{1} \\
w_{4}=0.1323941527885 \\
w_{3}=w_{4} \\
w_{6}=w_{4} \\
w_{7}=0.225\end{array}$ \\
\hline 13 puntos & 7 & & 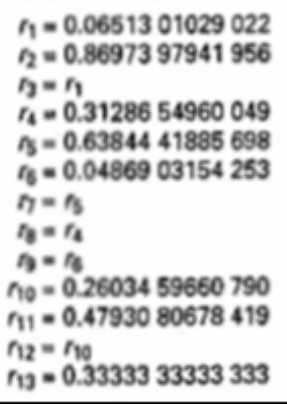 & 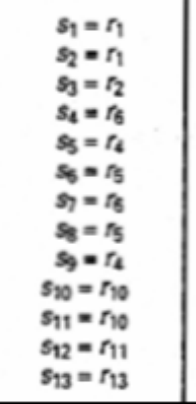 & $\begin{array}{l}w_{1}=0.0533472356088 \\
w_{2}=w_{1} \\
w_{2}=w_{1} \\
w_{4}=0.0771137608903 \\
w_{4}=w_{4} \\
w_{6}=w_{4} \\
w_{1}=w_{4} \\
w_{4}=w_{4} \\
w_{3}=w_{4} \\
w_{10}=0.1756152574332 \\
w_{11}=w_{10} \\
w_{12}=w_{10} \\
w_{13}=-0.1495700444677\end{array}$ \\
\hline
\end{tabular}

El método alfa $(\alpha)$ de integración numérica considera que la temperatura en el tiempo $t+\alpha \Delta t$ es:

$$
\dot{\mathrm{T}}^{\mathrm{t}+\alpha \Delta \mathrm{t}}=\frac{\mathrm{T}^{\mathrm{t}+\Delta t}-\mathrm{T}^{\mathrm{t}}}{\Delta \mathrm{t}}
$$

donde a es una constante cuyo valor determina la estabilidad y precisión de la solución. El desarrollo de Taylor de la $T^{t}$ alrededor del punto $t+\alpha \Delta t$ es:

$$
\mathrm{T}^{\mathrm{t}}=\mathrm{T}^{\mathrm{t}+\alpha \Delta \mathrm{t}}+\dot{\mathrm{T}}^{\mathrm{t}+\alpha \Delta \mathrm{t}}(-\alpha \Delta \mathrm{t})+\ddot{\mathrm{T}}^{\mathrm{t}+\alpha \Delta \mathrm{t}} \frac{(-\alpha \Delta \mathrm{t})^{2}}{2 !}+\ldots \ldots
$$

Si se consideran sólo los dos primeros términos del desarrollo (el error en el desarrollo de Taylor es del orden de $\Delta \mathrm{t}^{2}$ ) y se reemplaza $\dot{T}^{\text {t+ast }}$ utilizando 
la Ec. 2.49 se obtiene:

$\mathrm{T}^{\mathrm{t}+\alpha \Delta \mathrm{t}}=(1-\alpha) \mathrm{T}^{\mathrm{t}}+\alpha \mathrm{T}^{\mathrm{t}+\Delta \mathrm{t}}$

Reemplazando las Ec. 2.49 y 2.51 en la Ec. 2.31:

$\mathrm{CG}\left(\frac{\mathrm{T}^{\mathrm{t}+\Delta \mathrm{t}}-\mathrm{T}^{\mathrm{t}}}{\Delta \mathrm{t}}\right)+\mathrm{KG}\left((1-\alpha) \mathrm{T}^{\mathrm{t}}+\alpha \mathrm{T}^{\mathrm{t}+\Delta \mathrm{t}}\right)=\mathrm{FG}$

Reordenando:

$\left(\frac{\mathrm{CG}}{\Delta \mathrm{t}}+\alpha \mathrm{KG}\right) \mathrm{T}^{\mathrm{t}+\Delta \mathrm{t}}=\mathrm{FG}+\left(\frac{\mathrm{CG}}{\Delta \mathrm{t}}-(1-\alpha) \mathrm{KG}\right) \mathrm{T}^{\mathrm{t}}$

Las propiedades del tipo de integración numérica dependen del valor elegido del parámetro $a$. Los siguientes casos son los más utilizados y corresponden a $\alpha=0$ (método explícito) donde la $K G$ no interviene en el lado izquierdo de la Ec. 2.53. Este método, llamado también método explícito de Euler, presenta un error que se encuentra en el orden de $\Delta t$. Si $\alpha=1$ se lo denomina método implícito de Euler y la KG no interviene en el lado derecho de la igualdad (error en el orden de $\Delta \mathrm{t}$ ). En el caso que $\alpha=0.5$ se lo denomina caso semi-explícito o método de Crank-Nicolson y presenta una precisión de segundo orden en $\Delta$ t. Para un $\alpha \geq 0.5$ está demostrado que el método $\alpha$ es incondicionalmente estable (Bathe, 1996).

\subsection{Ensamble de las matrices elementales}

Como se explicó anteriormente el cálculo de las matrices elementales se realiza elemento a elemento y luego requiere de su ensamble en una matriz global. La malla utilizada para la discretización espacial contiene información de la conectividad de los nodos que componen cada elemento en la matriz que llamaremos tm (NODES). En el caso de un problema bidimensional discretizado con elementos triangulares, la dimensión de la matriz tm es de 
$\left[3 \times \mathrm{N}_{\mathrm{e}}\right.$ ] siendo $\mathrm{N}_{\mathrm{e}}$ el número total de elementos de la malla; cada columna de esa matriz corresponde a un elemento de la malla. Si tomamos un elemento cualquiera de una malla, por ejemplo el 4490 (Tabla 2-2). El triángulo o elemento 4490 está compuesto por tres nodos; el 66, 65, y 73 que corresponden a los nodos locales 1, 2, y 3 siguiendo el orden del triángulo de referencia. La matriz elemental de capacitancia se define como

$$
\mathrm{CG}_{\mathrm{ij}}^{\mathrm{e}=4490}=\int_{\mathrm{e}=4490} \mathrm{~h}_{\mathrm{i}} \rho \mathrm{Cp} \mathrm{h}_{\mathrm{j}} \mathrm{d} \Omega_{\mathrm{e}}
$$

donde i, j varían de 1 a 3 en la matriz local de cada elemento. Por otra parte para el ejemplo analizado el $\mathrm{C}_{1,1}$ corresponde en la matriz global a $\mathrm{C}_{66,66}$ y $\mathrm{C}_{1,3}$ corresponde a $\mathrm{C}_{66,73}$. Para un problema bidimensional utilizando funciones de forma lineales ( $h 1, h 2, y$ h3) las matrices elementales tienen todas dimensión [3 $\times 3$ ], y el vector de esfuerzos [3 x 1].

De la misma manera se realiza el cálculo y ensamble de las demás matrices: matriz de conductancia y el vector de esfuerzos.

Tabla 2-2 Matriz tm o NODES de la discretización en elementos triangulares

\begin{tabular}{|r|r|r|r|r|r|r|r|}
\hline \multicolumn{2}{|c|}{4487} & \multicolumn{1}{|c|}{4488} & \multicolumn{1}{c|}{4489} & 4490 & 4491 & 4492 & \multicolumn{1}{c|}{4493} \\
\hline 1 & 66 & 66 & 66 & 66 & 20 & 20 & 20 \\
\hline 2 & 12 & 65 & 73 & 65 & 74 & 74 & 7 \\
\hline 3 & 58 & 58 & 12 & 73 & 13 & 14 & 74 \\
\hline
\end{tabular}

\subsection{Desarrollo del programa computacional en elementos finitos}

De acuerdo a la formulación presentada se ha desarrollado un programa computacional en Matlab 6.5 para simular la transferencia de energía en estado no estacionario en 2 dimensiones considerando condiciones de borde 
de tipo Dirichlet o Neumann (condición de temperatura prescripta o presencia de coeficiente de transferencia calórica en la interfase respectivamente).

El Programa se presenta al final del Capítulo ( Apéndice 2.1).

\subsection{Validación del programa computacional en} problemas de transferencia de calor en sistemas bidimensionales con propiedades termofísicas constantes

Esta Sección se dedicará a la validación de los programas computacionales generados utilizando datos de literatura publicados en revistas internacionales.

El objetivo principal es asegurar que el método numérico, cuya teoría fue descrita en las Secciones 2.1 a 2.4, implementado en programas computacionales propios, obtenga resultados correctos cuando se simulen problemas de transferencia de calor en dominios bidimensionales.

\subsubsection{Simulación de problemas con condición de borde tipo Dirichlet (temperatura prescripta en el borde) \\ El programa computacional se implementó para simular la transferencia} de calor en estado no estacionario en un producto cárnico de sección transversal elíptica y sin efectos axiales ya que la longitud del mismo era mucho mayor que los ejes de la elipse. Se consideró por lo tanto un sistema bidimensional con propiedades termofísicas constantes y se plantearon condiciones de borde de temperatura prescripta. Se compararon las predicciones numéricas obtenidas en el presente trabajo de Tesis con resultados publicados en el trabajo "A Numerical Method for Simulating Heat and Mass Transfer in Heterogeneous and Irregularly Shaped Foodstuffs", 
(Califano and Zaritzky, 1993). En dicho trabajo se simuló el calentamiento de piezas cárnicas cuya sección transversal era de geometría elíptica, mediante el método numérico de volúmenes de control (VC) con grilla ajustada a los contornos (boundary-fitted grid, BFG). Las temperaturas prescriptas utilizadas fueron de $70,80,90$ y $100^{\circ} \mathrm{C}$ y se consideró temperatura inicial de $20{ }^{\circ} \mathrm{C}$. Según lo expuesto en la Sección 2.1, las ecuaciones que representan el modelo matemático (formulación fuerte) son:

$\rho \operatorname{Cp} \frac{\partial \mathrm{T}}{\partial \mathrm{t}}=\mathrm{k}\left(\frac{\partial^{2} \mathrm{~T}}{\partial \mathrm{x}^{2}}+\frac{\partial^{2} \mathrm{~T}}{\partial \mathrm{y}^{2}}\right)$

en $\Omega$

$\mathrm{t}=0 \quad \mathrm{~T}=\mathrm{T}_{0}$

en $\Omega$

$\mathrm{t}>0 \quad \mathrm{~T}=\mathrm{T}_{1}$

en $\delta \Omega_{2}$

La formulación variacional del problema aplicando elementos finitos en forma discretizada resulta en (forma débil):

$\left[\sum_{\mathrm{e}} \int_{\Omega_{\mathrm{e}}} \mathrm{H}^{\mathrm{t}} \rho \mathrm{CpHd} \Omega_{\mathrm{e}}\right] \dot{\mathrm{T}}+\left[\sum_{\mathrm{e}} \int_{\Omega_{\mathrm{e}}} \mathrm{B}^{\mathrm{t}} \mathrm{kBd} \Omega_{\mathrm{e}}\right] \mathrm{T}=0$

donde $C G=\sum_{e} \int_{\Omega_{e}} H^{t} \rho C p H d \Omega, \quad$ y $K G=\sum_{e} \int_{\Omega_{e}} B^{t} k B d \Omega_{e}$

Por lo tanto la ecuación 2.57 se puede escribir como:

$\mathrm{CG} \dot{\mathrm{T}}+\mathrm{KGT}=0$

Se puede observar que el término que representa la integral de línea (borde del dominio) desaparece debido a que en el problema propuesto las 
condiciones de contorno son de temperatura prescripta. La geometría utilizada en el trabajo analizado (Califano y Zaritzky,1993) se observa en la Fig. 2-4.

La malla utilizada aplicando el método de elementos finitos fue la misma que se utilizó en el Método de VC con BFG, sin embargo en la formulación variacional se requirió de la generación de la matriz NODES para calcular las matrices globales en el programa. En este caso se utilizaron elementos rectangulares isoparamétricos lineales.

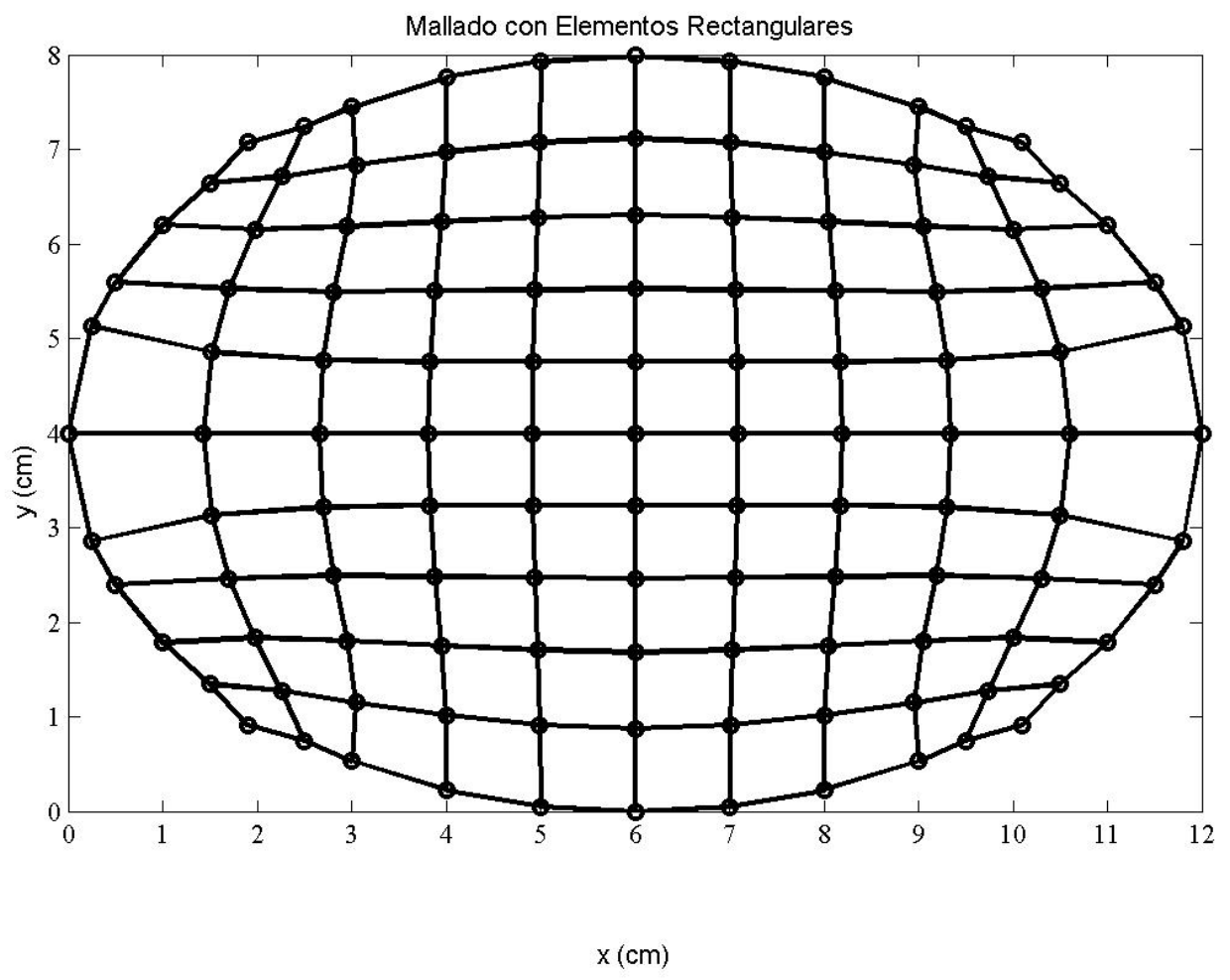

Fig. 2-4 Discretización del dominio elipsoidal en elementos rectangulares

Se utilizó el método de integración temporal descrito en la Sección 2.3 con $\alpha=1$, ya que es incondicionalmente estable y permite utilizar $\Delta \mathrm{t}$ más grandes.

La grilla utilizada consta de 121 puntos o nodos teniendo 4 nodos por elemento y un total de 100 elementos. 
La conductividad térmica, densidad y capacidad calorífica utilizadas fueron (Miles y col., 1983):

$$
\begin{aligned}
& \mathrm{k}=0.454 \mathrm{~W} / \mathrm{m} \mathrm{K}, \\
& \mathrm{C} p=3477.5 \mathrm{~J} / \mathrm{kg} \mathrm{K}, \\
& \rho=969.2 \mathrm{~kg} / \mathrm{m}^{3} .
\end{aligned}
$$

Se realizaron varias simulaciones para distintas temperaturas prescriptas y se compararon los tiempos de proceso (tiempo que tardó el alimento en alcanzar los $60^{\circ} \mathrm{C}$ en el centro térmico) con los predichos mediante el método de volúmenes de control (VC y BFG). Se utilizó el mismo incremento en el tiempo, $\Delta \mathrm{t}=4$ segundos, para ambos métodos.

Los resultados se muestran en la Tabla 2-3.

Tabla 2-3 Tiempos de calentamiento para que el alimento alcance los $60^{\circ} \mathrm{C}$ en el centro geométrico utilizando el MEF y VC con BFG

\begin{tabular}{|c|c|c|}
\hline Temperatura Prescripta $\left(^{\circ} \mathbf{C}\right)$ & \multicolumn{2}{|c|}{ Tiempo de calentamiento tc (min) } \\
\hline $\mathbf{T}_{\text {pres }}$ & Presente trabajo (MEF) & VC con BFG* \\
\hline 70 & 82.3 & 84.6 \\
\hline 80 & 61.9 & 63.3 \\
\hline 90 & 51.8 & 53.1 \\
\hline 100 & 45.5 & 46.6 \\
\hline
\end{tabular}

* datos de Califano y Zaritzky 1993. 
Se puede observar que el método utilizado en el presente trabajo de Tesis predice un tiempo tc menor que el BFG para que el alimento llegue a una temperatura de $60^{\circ} \mathrm{C}$ en el centro. Sin embargo las diferencias no son importantes, disminuyendo a medida que aumenta $T_{\text {pres. }}$ El error máximo se observa para el caso de $\mathrm{T}_{\text {pres }}=70^{\circ} \mathrm{C}$ con 2.3 minutos (error relativo menor al $3 \%$ ). En las Fig.2-5 a) b) y c) se muestran gráficos de Temperatura a distintos tiempos correspondientes a una temperatura prescripta de $70^{\circ} \mathrm{C}$.

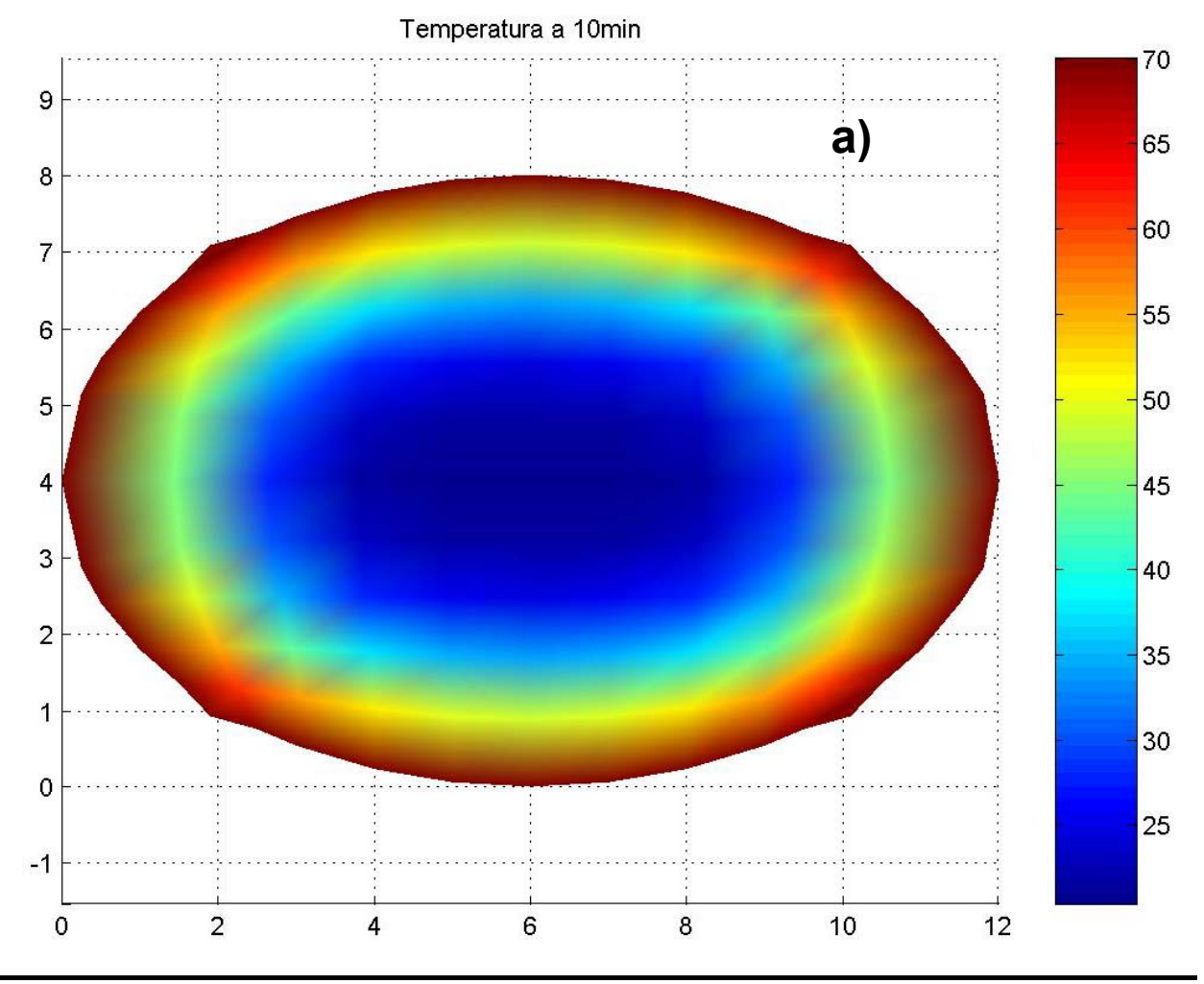

Fig. 2-5 a) Perfil de temperatura luego de 10 minutos de calentamiento 


\section{(F)}

C I D C A
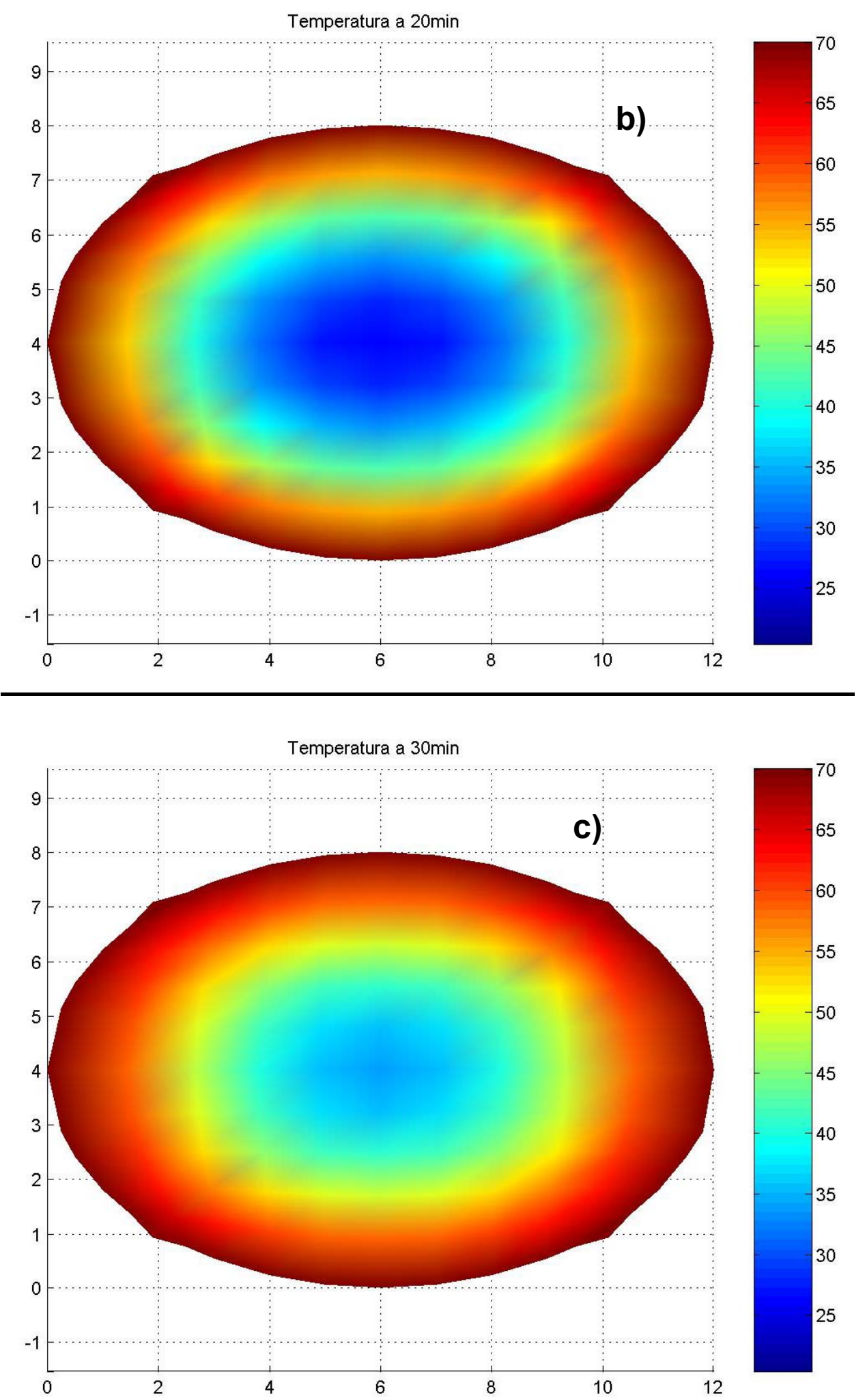

Fig. 2-5 Perfil de temperatura luego de b) 20, y c) 30 minutos de calentamiento 
En la Fig. 2-6 se muestra a modo de ejemplo la temperatura en función del tiempo en el centro térmico para distintas temperaturas prescriptas.

Adicionalmente se comparó con el simulador comercial COMSOL, AB (2005). Se puede observar en la Fig. 2.8 que existe una buena concordancia entre las predicciones, aunque el sistema de mallado (discretización) en los dos casos fue diferente. En el caso del código computacional propio la discretización fue en rectángulos (100 rectángulos) (similarmente al trabajo de grilla ajustada a los contornos ) y en el caso del simulador comercial fueron triángulos (474), los cuales se ajustan mejor a las geometrías curvas e irregulares. Sin embargo el programa desarrollado en el presente trabajo de Tesis utilizó menor cantidad de elementos llegando a resultados similares. La Fig. 2-7 y 2-8 permite observar la grilla utilizada para la resolución del problema por COMSOL y el gráfico comparativo.

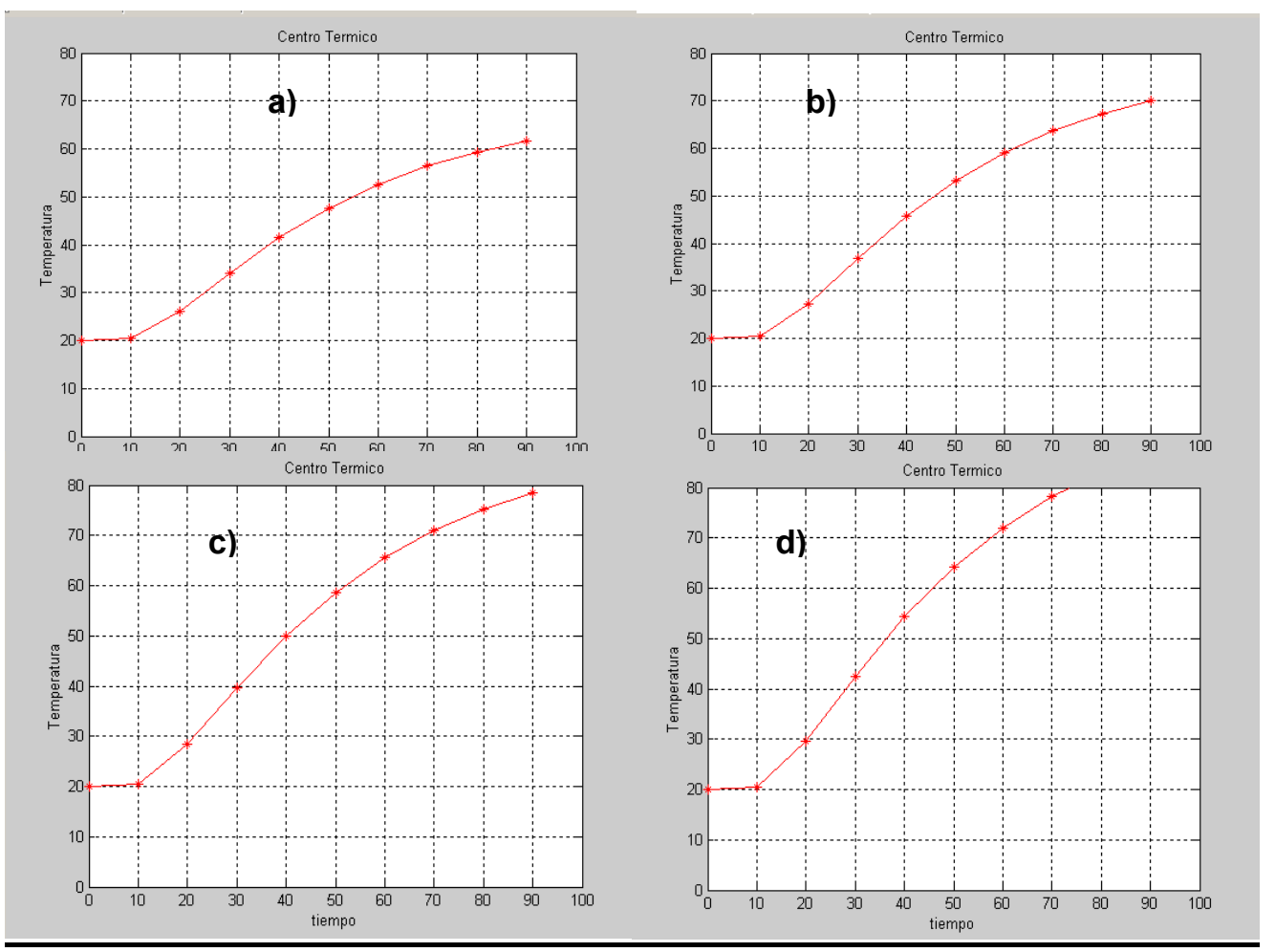

Fig. 2-6 Temperatura en función del tiempo en el centro térmico para distintas temperaturas de fluido, a) $\mathrm{T}_{\text {pres }}=70^{\circ} \mathrm{C}$, b) $\mathrm{T}_{\text {pres }}=80^{\circ} \mathrm{C}$, c) $\mathrm{T}_{\text {pres }}=90^{\circ} \mathrm{C}$ y d) $\mathrm{T}_{\text {pres }}=100^{\circ} \mathrm{C}$. 


\section{I D C A}

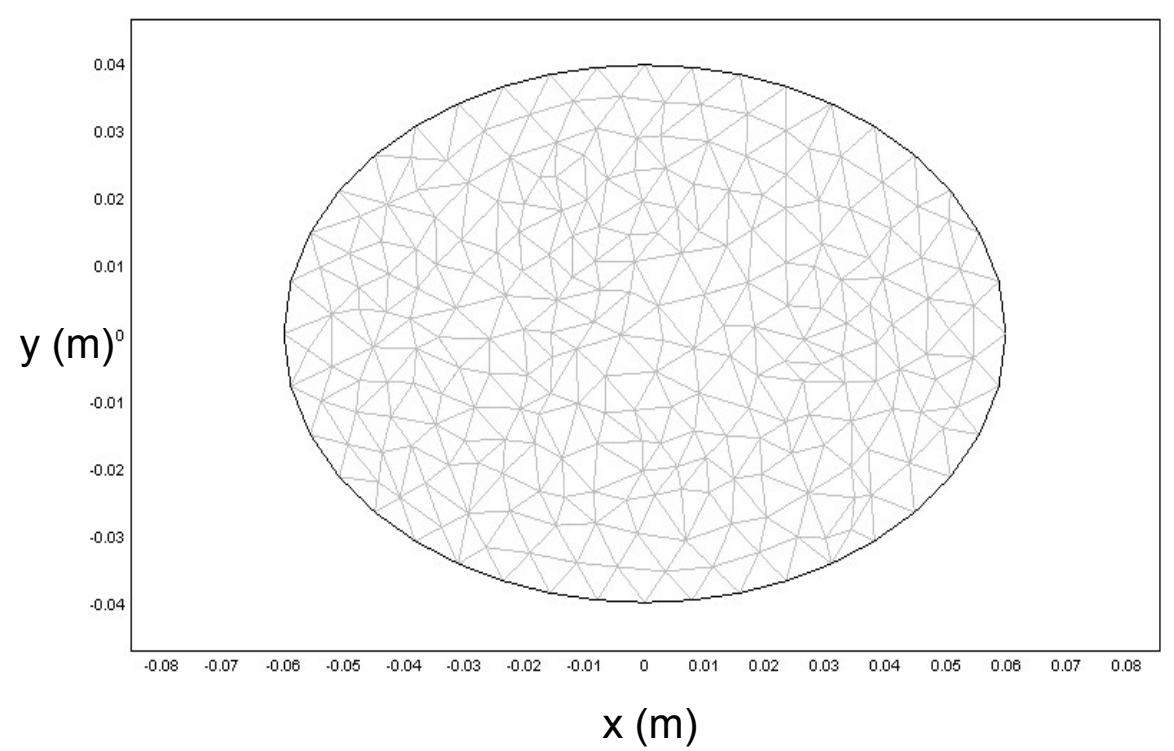

Fig. 2-7 Mallado de la geometría bidimensional generada en COMSOL

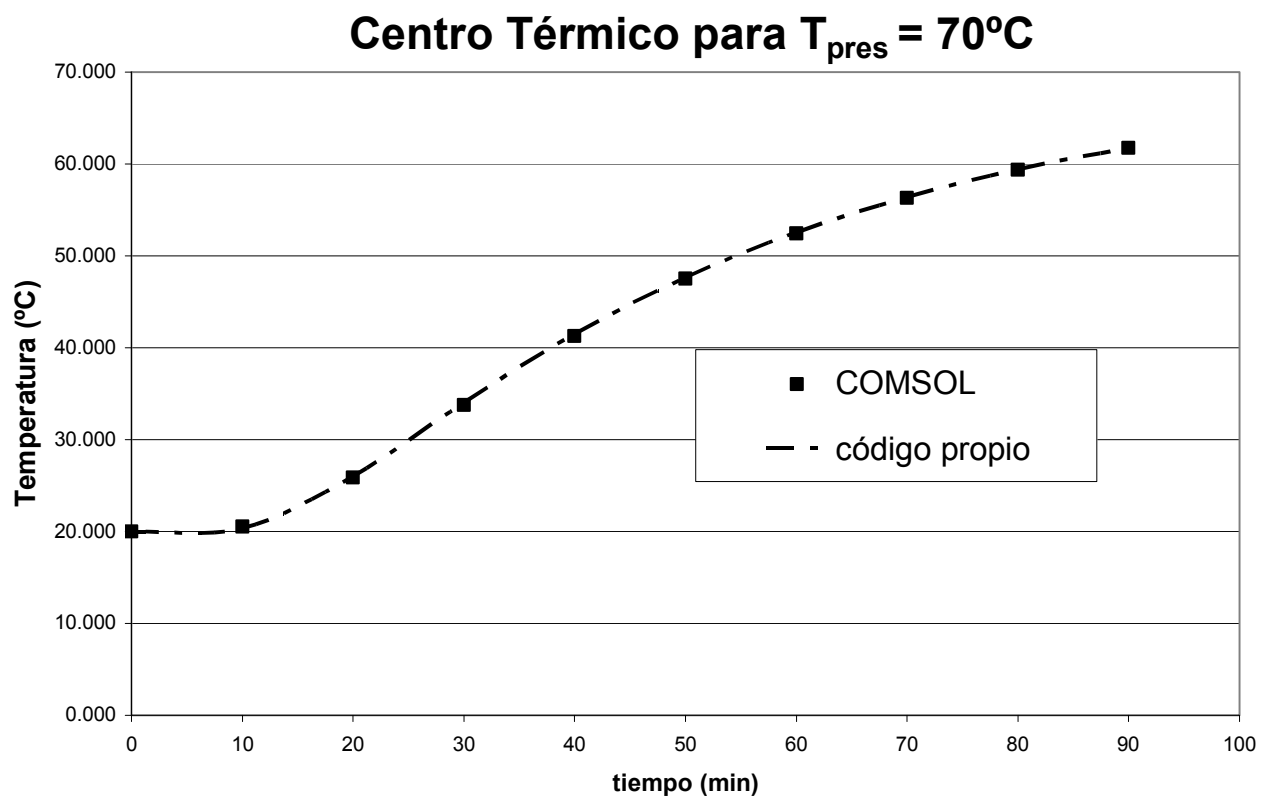

Fig. 2-8 Temperatura en función del tiempo en el centro térmico utilizando el código abierto propio y un paquete comercial de simulación que utiliza el MEF (COMSOL). La temperatura inicial es de $20^{\circ} \mathrm{C}$ y una temperatura externa del fluido, $T_{\text {pres }}=70^{\circ} \mathrm{C}$. 


\subsubsection{Simulación de problemas con condición de borde convectiva}

El modelo matemático planteado es:

$$
\begin{array}{ll}
\rho \operatorname{Cp} \frac{\partial T}{\partial t}=k\left(\frac{\partial^{2} T}{\partial x^{2}}+\frac{\partial^{2} T}{\partial y^{2}}\right) & \text { en } \Omega \\
T=T_{0} & \text { a } t=0 \\
-k\left(\frac{\partial T}{\partial x} \cdot n_{x}+\frac{\partial T}{\partial y} \cdot n_{y}\right)=h\left(T-T_{\text {ext }}\right) & \text { a } t \geq 0 \quad \text { en } \delta \Omega_{1}
\end{array}
$$

La formulación variacional del problema aplicando elementos finitos en forma discretizada resulta (forma débil):

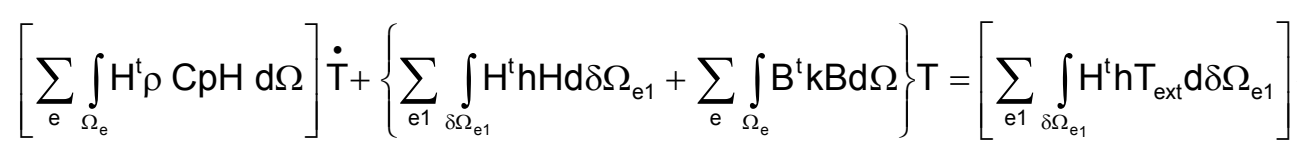

definiendo

$$
\mathrm{CG}=\sum_{\mathrm{e}} \int_{\Omega_{\mathrm{e}}} \mathrm{H}^{\mathrm{t}} \rho \mathrm{Cp} \cdot \mathrm{Hd} \Omega \quad \text { : la matriz global de capacitancia, }
$$$$
\mathrm{KG}=\sum_{\mathrm{e} 1} \int_{\delta \Omega_{\mathrm{e}}} \mathrm{H}^{\mathrm{t}} \mathrm{hHd} \delta \Omega_{\mathrm{e} 1}+\sum_{\mathrm{e}} \int_{\Omega_{\mathrm{e}}} \mathrm{B}^{\mathrm{t}} \mathrm{kBd} \Omega \quad \text { : la matriz global de conductancia, }
$$$$
\mathrm{FG}=\sum_{\mathrm{e} 1} \int_{\delta \Omega_{\mathrm{e} 1}} \mathrm{H}^{\mathrm{t} h \mathrm{ext}} \mathrm{T}_{\mathrm{d}} \mathrm{d} \delta \Omega_{\mathrm{e} 1} \quad: \text { : el vector de fuerzas globales, }
$$

la ecuación 2.62 se escribe como CG $\cdot \dot{\mathrm{T}}+\mathrm{KG} \cdot \mathrm{T}=\mathrm{FG}$. 
Se puede observar la aparición de un vector global de fuerzas debido a la incorporación de la condición de borde tipo convectiva. Por la misma razón la matriz global de conductancia presenta un término adicional que involucra la integral de borde.

Se utilizó el mismo método de discretización temporal que en el caso de temperatura prescripta.

Se simuló el calentamiento de un alimento cárneo de iguales propiedades térmicas y geometría que el expuesto en la Sección 2.6.1, con la única diferencia que en este caso se utilizó una condición de borde con convectiva con un coeficiente de transferencia calórica $h=300 \mathrm{~W} / \mathrm{m}^{2 \circ} \mathrm{C}$.

En la Tabla 2-4 se muestran los tiempos de calentamiento para que el centro alcance los $60^{\circ} \mathrm{C}$ para distintas temperaturas externas del fluido.

Tabla 2-4 Tiempos de calentamiento para que el alimento alcance los $60^{\circ} \mathrm{C}$ en el centro geométrico utilizando el MEF y VC con BFG

\begin{tabular}{|c|c|c|}
\hline Temperatura Externa $\left(^{\circ} \mathrm{C}\right)$ & \multicolumn{2}{|c|}{ Tiempo de calentamiento tc (min) } \\
\hline $\mathbf{T}_{\text {ext }}$ & Presente trabajo (MEF) & VC con BFG* \\
\hline 70 & 90.4 & 91.4 \\
\hline 80 & 68.3 & 68.7 \\
\hline 90 & 57.3 & 57.4 \\
\hline 100 & 50.4 & 50.3 \\
\hline
\end{tabular}

* datos de Califano y Zaritzky, 1993.

Se puede observar que existe una excelente concordancia entre los tiempos predichos para ambos métodos por lo que se puede concluir que el programa computacional funciona de manera correcta utilizando condiciones de contorno convectivas (error relativo máximo menor al 1.2\%). En las Fig.2-9 a) y b) se muestran gráficos de temperatura a distintos tiempos correspondientes a una temperatura externa $\mathrm{T}_{\text {ext }}=100^{\circ} \mathrm{C}$ y $\mathrm{h}=300 \mathrm{~W} / \mathrm{m}^{2}{ }^{\circ} \mathrm{C}$. 

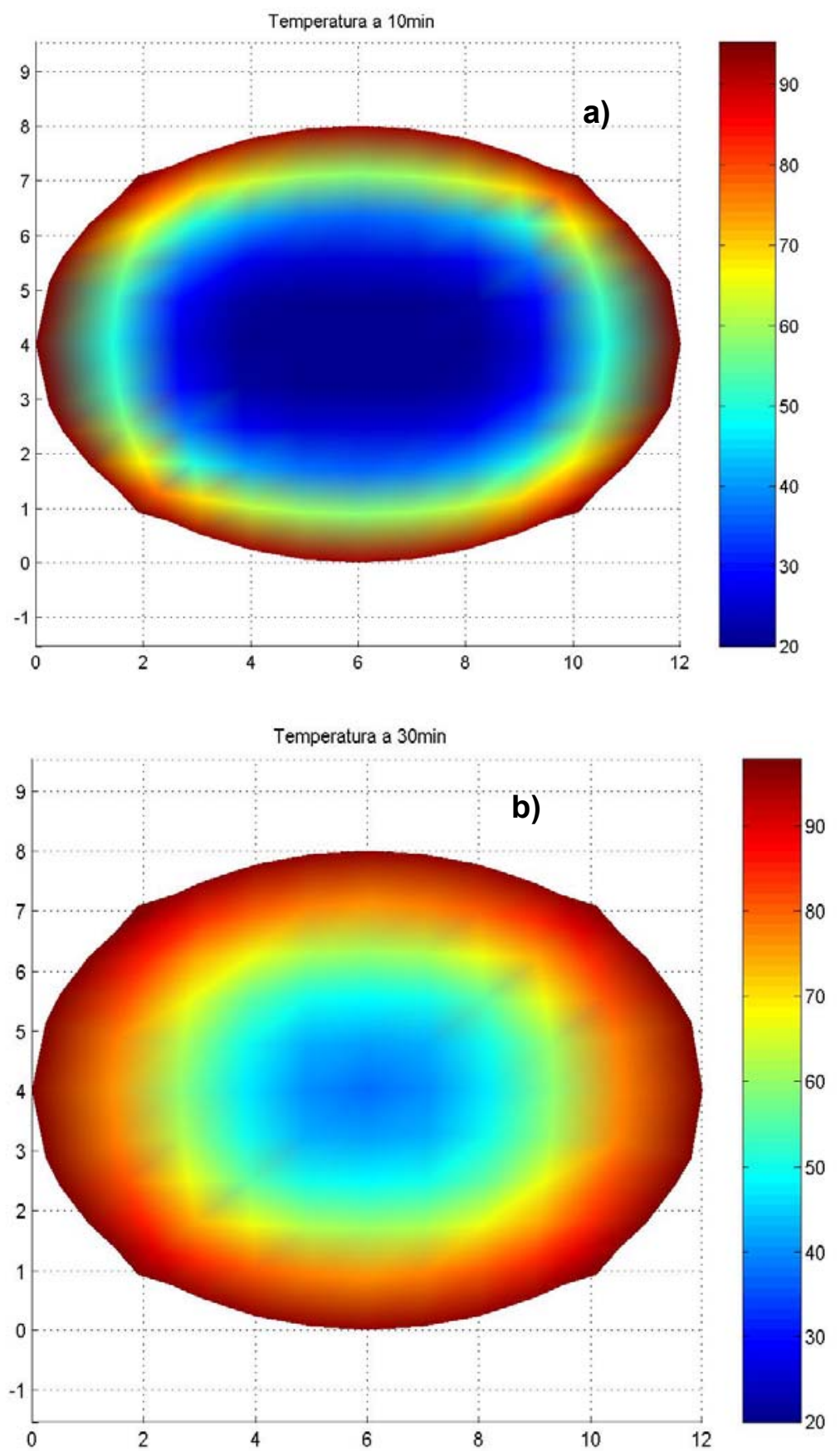

Fig. 2-9 Perfil de temperaturas luego de a) 10 minutos y b) $\mathbf{3 0}$ minutos de calentamiento en un fluido con $\mathrm{T}_{\text {ext }}=100^{\circ} \mathrm{C}$ y con un coeficiente de transferencia calórica de $300 \mathrm{~W} / \mathrm{m}^{2}{ }^{\circ} \mathrm{C}$

La Fig. 2-10 muestra la temperatura en función del tiempo en el centro térmico para un $\mathrm{h}=50 \mathrm{~W} / \mathrm{m}^{2 \circ} \mathrm{C}$ utilizando el MEF y VC con BFG. La temperatura inicial es de $20^{\circ} \mathrm{C}$ y la temperatura externa del fluido, $\mathrm{T}_{\text {ext }}=70^{\circ} \mathrm{C}$. Se puede 
observar que los datos numéricos son concordantes dando un error promedio porcentual relativo menor al $1 \%$.

Centro Térmico para un $\mathrm{h}=50 \mathrm{~W} / \mathrm{m}^{2}{ }^{\circ} \mathrm{C}$

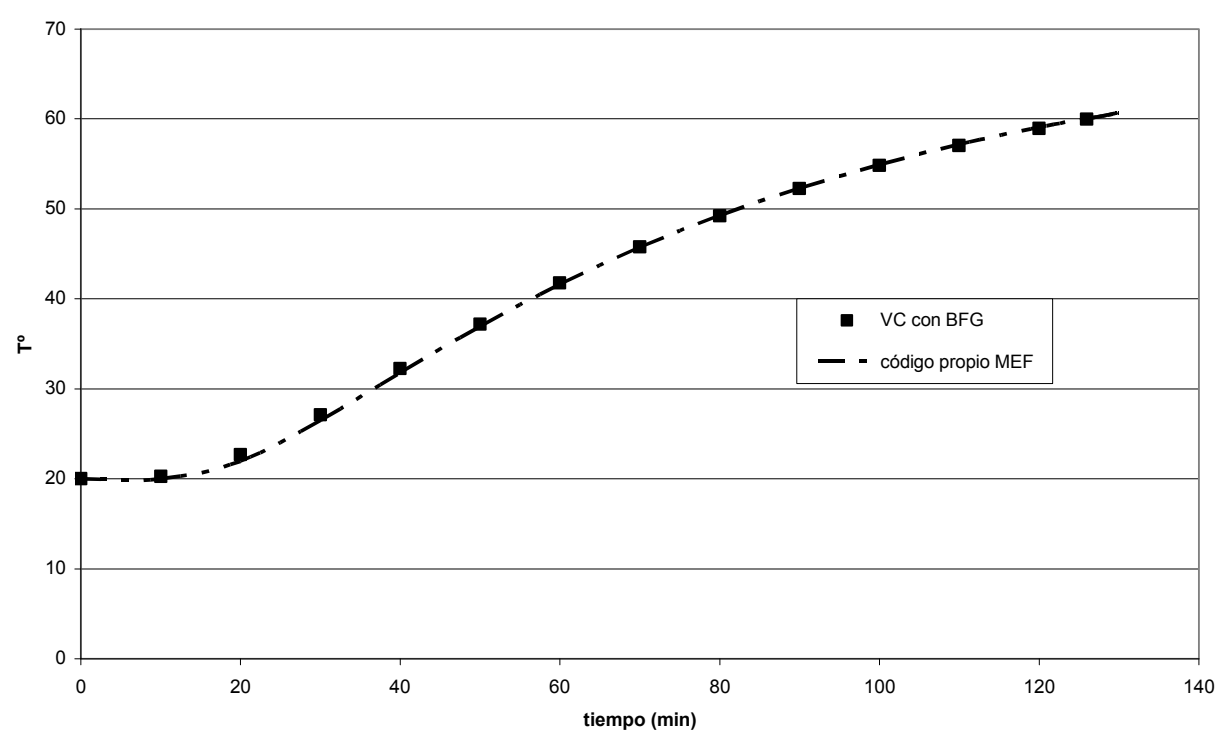

Fig. 2-10 Temperatura en función del tiempo en el centro térmico utilizando el MEF en un código abierto y utilizando volúmenes de control con grilla ajustada a los contornos (VC con BFG) para un coeficiente de transferencia calórico de $50 \mathrm{~W} / \mathrm{m}^{2}{ }^{\circ} \mathrm{C}$. La temperatura inicial es de $20^{\circ} \mathrm{C}$ y la $\mathrm{T}_{\text {ext }}=70^{\circ} \mathrm{C}$

\subsection{Conclusiones del Capítulo}

El programa computacional implementado simula el proceso de transferencia de calor en geometrías bidimensionales con temperatura prescripta y condiciones de contorno convectiva, con una excelente concordancia entre las predicciones obtenidas y los resultados obtenidos a través de: a) perfiles de temperatura informados en la literatura b) un paquete comercial, que utiliza el mismo método numérico. 


\title{
Apéndice 2-1
}

\section{Código Computacional 2D desarrollado en Matlab 6.5}

\subsubsection{Preprocesamiento}

\author{
grilla=fem.mesh.p'; \\ nodes $=$ fem.mesh.t; \\ borde=fem.mesh.e; \\ $\mathrm{n}=$ length(borde); \\ n2=length(nodes);
}

\%Lado 1 comprende nodos 1 y 2

\%Lado 2 comprende nodos 2 y 3

\%Lado 3 comprende nodos 3 y 1

$$
\begin{aligned}
& \text { \%\%\%\%\%\%\%\%\%\%\%\%1 } \\
& \text { if } \operatorname{nodes}(1, \mathrm{i})==\operatorname{borde} 2(1, \mathrm{k}) \\
& \text { if nodes }(2, i)==\operatorname{borde} 2(2, k) \\
& \text { elementoborde }(1, \mathrm{k})=1 \text {; } \\
& \text { elementoborde }(2, \mathrm{k})=0 \text {; } \\
& \text { elementoborde }(3, \mathrm{k})=0 \text {; } \\
& \text { elementoborde }(4, \mathrm{k})=\mathrm{i} \text {; } \\
& \text { end } \\
& \text { end } \\
& \text { \%\%\%\%\%\%\%\%\%\%2 } \\
& \text { if } \operatorname{nodes}(1, \mathrm{i})==\operatorname{borde} 2(1, \mathrm{k}) \\
& \text { if } \operatorname{nodes}(3, i)==\operatorname{borde} 2(2, k) \\
& \text { elementoborde }(1, \mathrm{k})=0 \text {; } \\
& \text { elementoborde }(2, \mathrm{k})=0 \text {; } \\
& \text { elementoborde }(3, \mathrm{k})=1 \text {; }
\end{aligned}
$$


elementoborde $(4, \mathrm{k})=\mathrm{i}$;

end

end

\%\%\%\%\%\%\%\%\%\%\%\%\%\%\%\%3

if nodes $(2, \mathrm{i})==$ borde2 $(1, \mathrm{k})$

if $\operatorname{nodes}(1, i)==\operatorname{borde} 2(2, k)$

elementoborde $(1, \mathrm{k})=1$;

elementoborde $(2, k)=0$;

elementoborde $(3, \mathrm{k})=0$;

elementoborde $(4, k)=i$;

end

end

$\% \% \% \% \% \% \% \% \% \% \% \% 4$

if nodes $(3, i)==$ borde $2(1, k)$

if $\operatorname{nodes}(1, \mathrm{i})==\operatorname{borde} 2(2, \mathrm{k})$

elementoborde $(1, \mathrm{k})=0$;

elementoborde $(2, \mathrm{k})=0$;

elementoborde $(3, k)=1$;

elementoborde $(4, \mathrm{k})=\mathrm{i}$;

end

end

\%\%\%\%\%\%\%\%\%\%\%\%\%\%\%5

if nodes $(2, \mathrm{i})==$ borde $2(1, \mathrm{k})$

if $\operatorname{nodes}(3, \mathrm{i})==\operatorname{borde} 2(2, \mathrm{k})$

elementoborde $(1, k)=0$;

elementoborde $(2, \mathrm{k})=1$;

elementoborde $(3, k)=0$;

elementoborde $(4, \mathrm{k})=\mathrm{i}$;

end 
end

end

end

save malla1.mat

\subsubsection{Programa principal}

clear all,clc

tcpu $0=$ cputime;

load malla1.mat;

\%matriz de coordenadas denodosc

coordenadas=grilla';

$\% \%$ numero de nodos totales

nodos_t=length(grilla);

\%numero de elementos

n_elem=length(nodes);

\%numero de nodos por elemento

nn_elem=3;

\%grados de libertad por elemento

gr_l=1;

\%alfa $=0$ es explicito, alfa $=1$ es implícito, alfa $=0.5$ es shem implícito o Crank Nicolson

alfa=1;

\%Inicialización de variables\%

Área=0;

LongAna=0;

LongNum=0;

$K G=\operatorname{sparse}(z e r o s($ nodos_t));

$\mathrm{CG}=$ sparse(zeros(nodos_t));

$\mathrm{FG}=$ sparse(zeros(nodos_t,1)); 
$\mathrm{T}=$ ones $($ nodos_t $\mathrm{t}, 1)$;

$\%$ Temperatura inicial

$\mathrm{Ti}=17$;

$\mathrm{T}=\mathrm{Ti}{ }^{*} \mathrm{~T}$;

\%Temperatura del fluido

$\mathrm{Tf}=66$;

\%Coeficiente de transferencia de calor

$\mathrm{h}=1615$;

\%Puntos de Integración gaussiana

$\mathrm{pc}=3$;

if $\mathrm{pc}==3$

$\mathrm{rg}=[15 / 90$ 6/9 15/90];

$\mathrm{sg}=[15 / 90$ 15/90 6/9];

$p=\left[\begin{array}{lll}1 / 3 & 1 / 3 & 1 / 3\end{array}\right]$;

end

$\mathrm{pp}=4$;

if $p p==2$

$r 1=[-\operatorname{sqrt}(1 / 3), \operatorname{sqrt}(1 / 3)]$;

$p 1=[1,1]$;

elseif $p p==3$

$\mathrm{r} 1=[-\operatorname{sqrt}(3 / 5), 0 ., \operatorname{sqrt}(3 / 5)]$;

$\mathrm{p} 1=[5 . / 9 ., 8 . / 9 ., 5 . / 9$.$] ;$

elseif $\mathrm{pp}==4$

$\mathrm{r} 1=[-0.861136311594053,-$

0.339981043584856,0.339981043584856,0.861136311594053];

p1 $=[0.347854845137454,0.652145154862546,0.652145154862546,0.3478548$ 45137454];

end

$\mathrm{s} 1=\mathrm{r} 1$; 


$$
\begin{aligned}
& \text { for } n=1: n \_ \text {elem; } \\
& \begin{array}{c}
\mathrm{kon}=0.5 \\
\mathrm{cp}=3437 \\
\mathrm{ro}=1000
\end{array}
\end{aligned}
$$

$\mathrm{KE}=z e r o s\left(g r \_{ }^{*} \mathrm{nn} \_\right.$elem);

$\mathrm{CE}=z e r o s(g r$ _l*nn_elem);

$\mathrm{KQ}=z e r o s(g r$ _l*nn_elem);

$\mathrm{F}=$ zeros $\left(g r \_{ }^{*} \mathrm{nn} \_\right.$elem,1);

KEtot=zeros(gr_I*nn_elem);

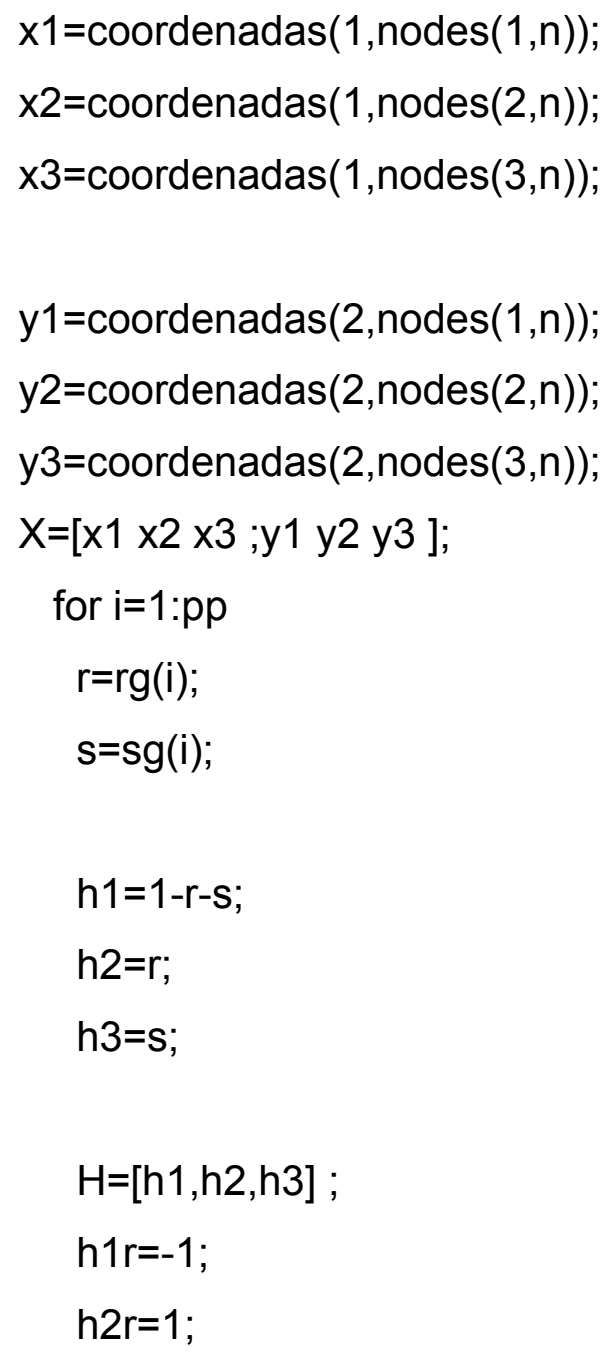


$\mathrm{h} 3 \mathrm{r}=0$;

h1s=-1;

$h 2 s=0$;

h3s=1;

$H r=[h 1 r, h 2 r, h 3 r ; h 1 s, h 2 s, h 3 s]$;

$\mathrm{J}=\mathrm{X}^{*} \mathrm{Hr}^{\prime}$;

$\operatorname{dete}=\operatorname{abs}(\operatorname{det}(\mathrm{J}))$;

$\mathrm{Ji}=\operatorname{inv}(\mathrm{J})$;

$B E=J i i^{*} \mathrm{Hr}$;

$\mathrm{w}=\mathrm{p}(\mathrm{i})$;

$\mathrm{KE}=\mathrm{KE}+0.5^{*} \mathrm{dete}^{*} \mathrm{~W}^{*} \mathrm{BE}^{* *} \mathrm{BE}{ }^{*} \mathrm{kon}$;

$\mathrm{CE}=\mathrm{CE}+0.5^{*}$ dete ${ }^{*} \mathrm{~W}^{*} \mathrm{H}^{*} \mathrm{H}^{*} \mathrm{ro}{ }^{*} \mathrm{cp}$;

Área=Área+0.5*dete* $\mathrm{w}$;

end

Te=Tf*ones $(\mathrm{nn}$ _elem,1);

for $m=1$ :length(elementoborde)

if $n==$ elementoborde $(4, m)$

if elementoborde $(1, \mathrm{~m})==1$

Long=0;

$\mathrm{m} 1=(\mathrm{y} 2-\mathrm{y} 1)$;

$\mathrm{m} 2=(\mathrm{x} 2-\mathrm{x} 1)$;

if $(\operatorname{abs}(\mathrm{m} 2)<=1 \mathrm{E}-10)$

aux=1;

LongAna=LongAna+abs $((y 2-y 1))^{*} a u x ;$

end

if $(\operatorname{abs}(\mathrm{m} 2)>=1 \mathrm{E}-10)$

$\mathrm{m} 3=\mathrm{m} 1 / \mathrm{m} 2$;

aux $=\operatorname{sqrt}\left(1+m 3^{\wedge} 2\right)$; 


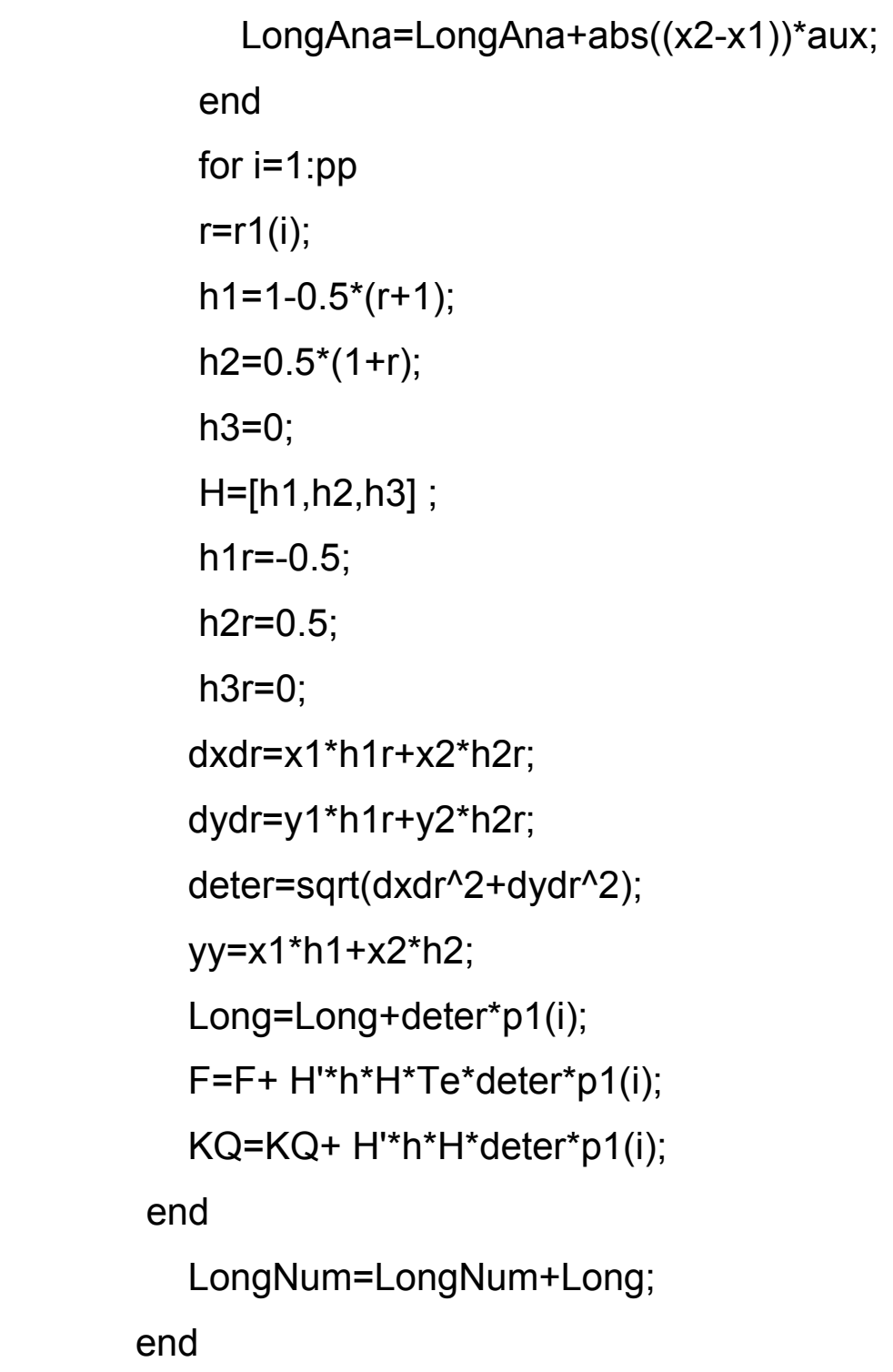

if elementoborde $(2, \mathrm{~m})==1$

Long $=0$;

$\mathrm{m} 1=(\mathrm{y} 2-\mathrm{y} 3)$;

$\mathrm{m} 2=(\mathrm{x} 2-\mathrm{x} 3)$;

if $(\operatorname{abs}(\mathrm{m} 2)<=1 \mathrm{E}-10)$

aux=1;

LongAna=LongAna+abs $((y 2-y 3))^{*} a u x$;

end 


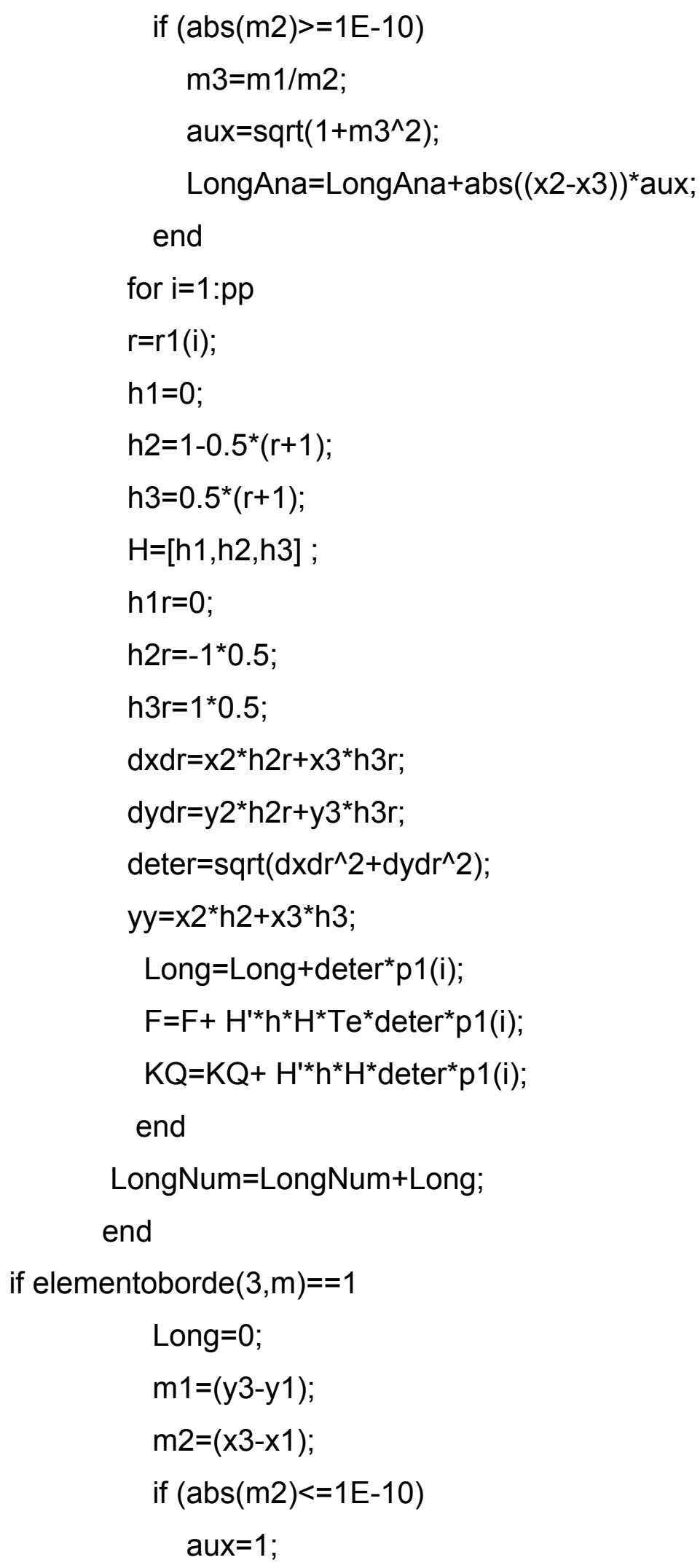




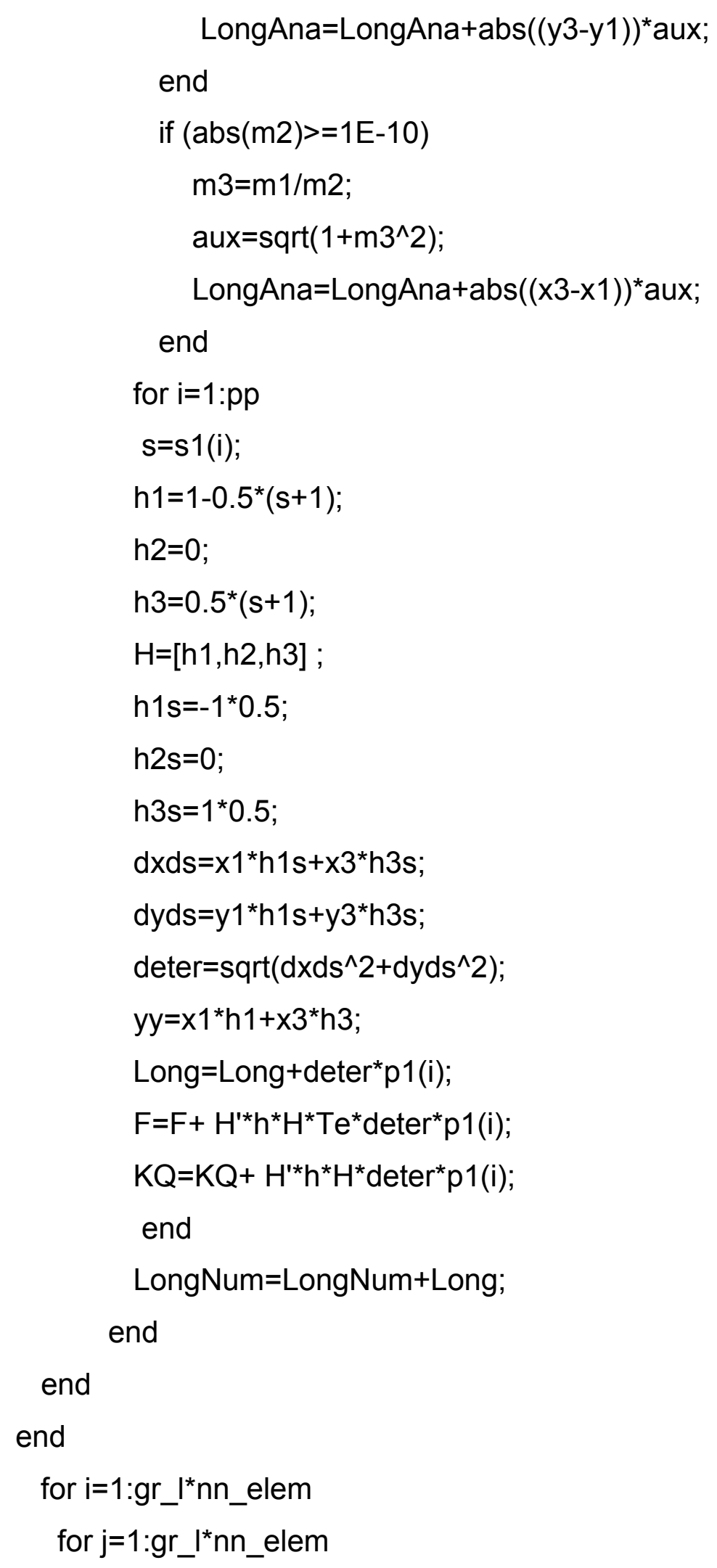




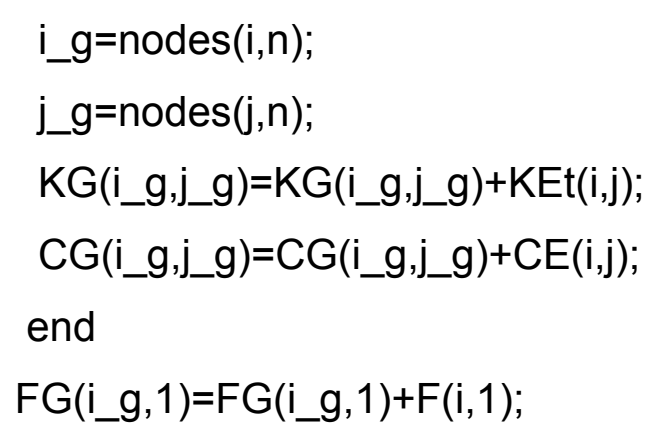


Qp $($ nciclo, 1)=tciclo;

$\mathrm{Qp}($ nciclo,2) $=\mathrm{Q}$;

$\mathrm{Qp}($ nciclo,3)=Qh;

long $x=0.1$;

ndiv=2;

for $i=1: n d i v+1$

punto=long $\mathrm{x}+(\mathrm{i}-1)^{\star}$ long $\mathrm{x} / \mathrm{ndiv}$;

$\mathrm{x}=$ punto;

$y=0.05$;

[h1,h2,h3, nodo1, nodo2, nodo3]=pospro2d(x,y,grilla, nodes);

Tpunto $($ nciclo, $\mathrm{i})=\mathrm{T}($ nodo 1$){ }^{\star} \mathrm{h} 1+\mathrm{T}($ nodo 2$){ }^{\star} \mathrm{h} 2+\mathrm{T}(\text { nodo3 })^{\star} \mathrm{h} 3$;

end

else

continue

end

nciclo $=$ nciclo +1

tcpu2=(cputime-tcpu0)/60

end

tcpu3 $=($ cputime-tcpu0 $) / 60$

Tpunto(2:end+1,::)=Tpunto(1:end,:);

Tpunto $(1,:)=\mathrm{Ti}$;

tpunto(2:end+1)=tpunto (1:end);

tpunto $(1)=0$;

plot(tpunto,Tpunto(:,1),tpunto,Tpunto(:,end))

xlabel('tiempo')

ylabel('Temperatura')

Title('Centro Térmico')

grid on

legend('Tcentro','Tborde');

tpunto=tpunto'; 


\subsubsection{Posprocesamiento}

function [h1,h2,h3, nodo1, nodo2, nodo3]=pospro2d(x,y,grilla,nodes)

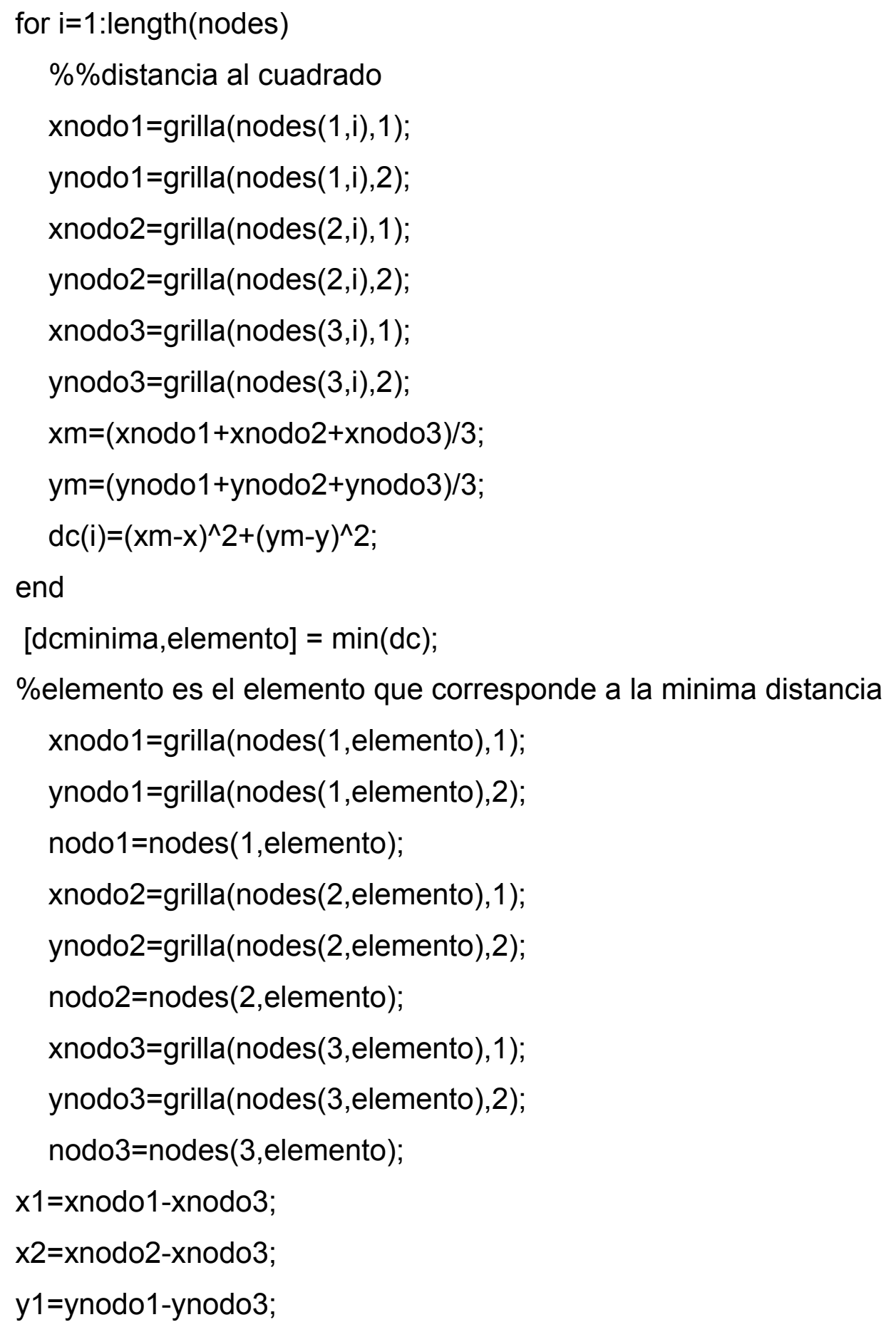


y2=ynodo2-ynodo3;

$\mathrm{A}=[\mathrm{x} 1 \mathrm{x} 2 ; \mathrm{y} 1 \mathrm{y} 2]$;

$\mathrm{H}=\operatorname{inv}(\mathrm{A})^{*}[\mathrm{x}-\mathrm{xnodo3} ; \mathrm{y}-\mathrm{ynodo}]$;

$\mathrm{h} 1=\mathrm{H}(1,1)$;

$\mathrm{h} 2=\mathrm{H}(2,1)$;

h3=1-h1-h2;

$\% \% \%$ compruebo que obtengo el punto original

$\mathrm{x}=$ xnodo1*h1+xnodo2*h2+xnodo3*h3;

y=ynodo1*h1+ynodo2*h2+ynodo3*h3;

\subsubsection{Flujos 2D}

function[Tf,Q,Qh]=flujos2D(kon,n_elem, elementoborde,nodes,T,coordenadas,p $\mathrm{p}, \mathrm{p} 1, \mathrm{r} 1, \mathrm{Tf}, \mathrm{h})$

$\mathrm{Q}=0$;

$\mathrm{Qh}=0$;

$[\mathrm{xcg}, \mathrm{ycg}]=$ CentroGravedad2D(coordenadas, nodes $)$

$\mathrm{x} 0=\mathrm{xcg}$;

y0=ycg;

for $n=1: n \_$elem

for $m=1$ :length(elementoborde)

if $\mathrm{n}==$ elementoborde $(4, \mathrm{~m})$

$x 1=\operatorname{coordenadas}(1, \operatorname{nodes}(1, n))$;

$x 2=\operatorname{coordenadas}(1, \operatorname{nodes}(2, n))$;

$x 3=\operatorname{coordenadas}(1, \operatorname{nodes}(3, n))$;

y1=coordenadas $(2, \operatorname{nodes}(1, n))$;

y2=coordenadas $(2, \operatorname{nodes}(2, n))$;

y3=coordenadas $(2, \operatorname{nodes}(3, n))$;

$\mathrm{X}=[\mathrm{x} 1 \mathrm{x} 2 \mathrm{x} 3$;y1 y2 y3 ]';

$\mathrm{h} 1 \mathrm{r}=1$;

$\mathrm{h} 2 \mathrm{r}=1$; 


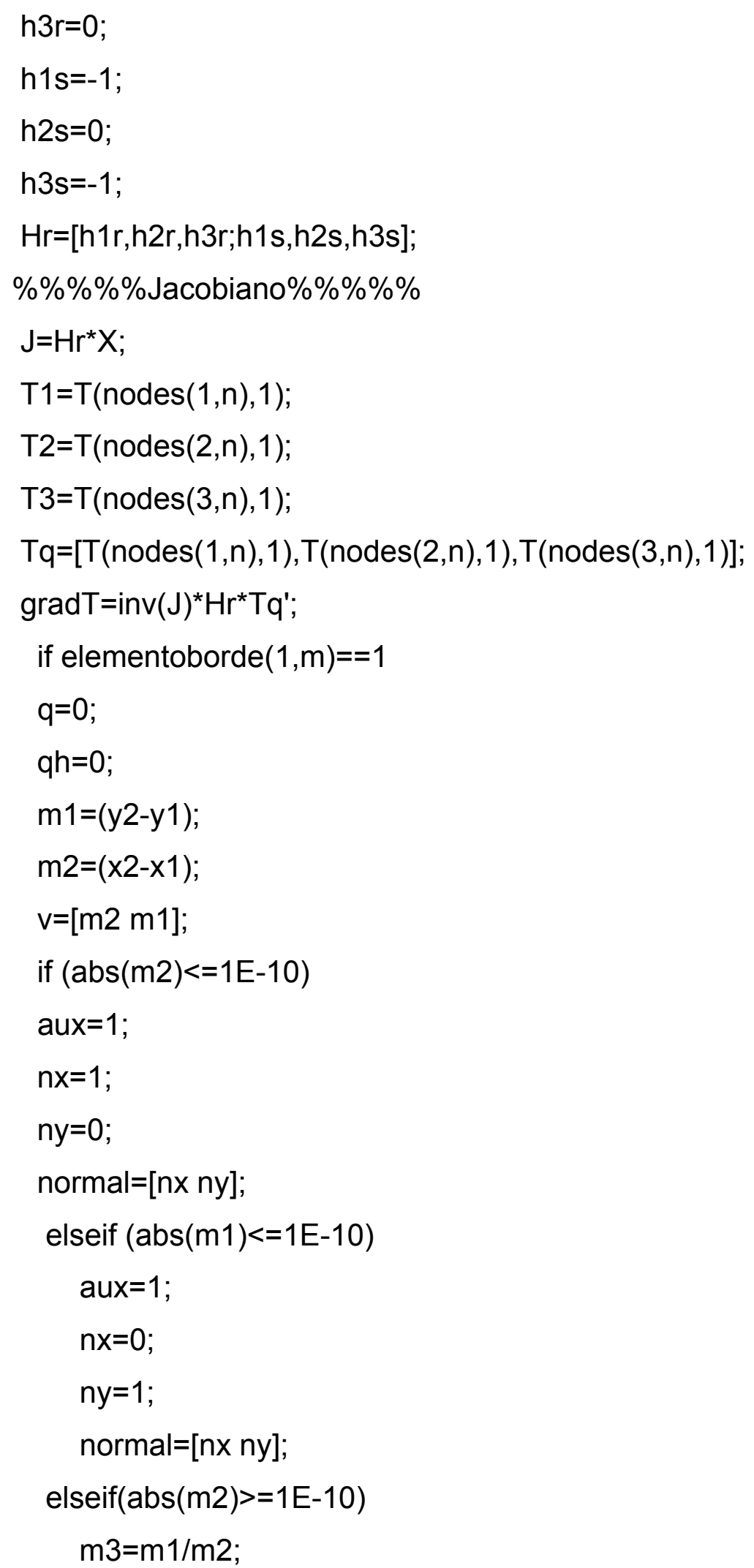




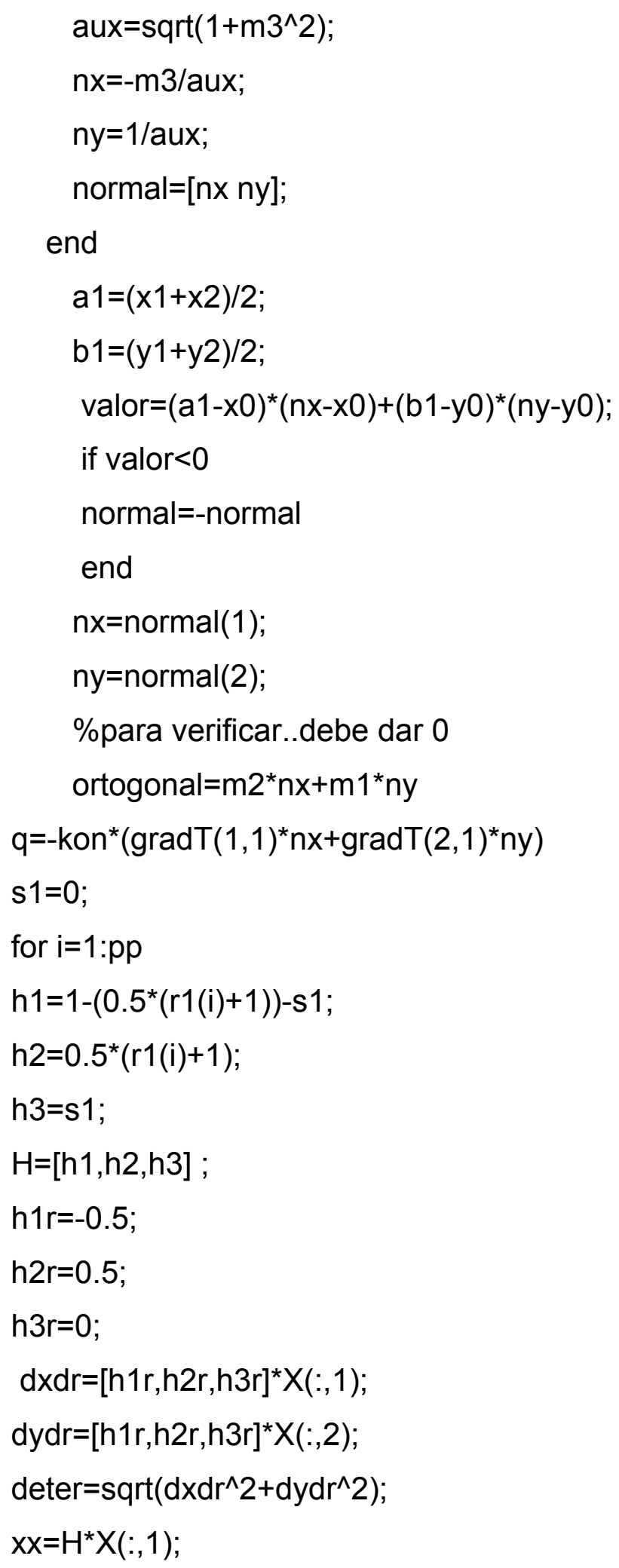




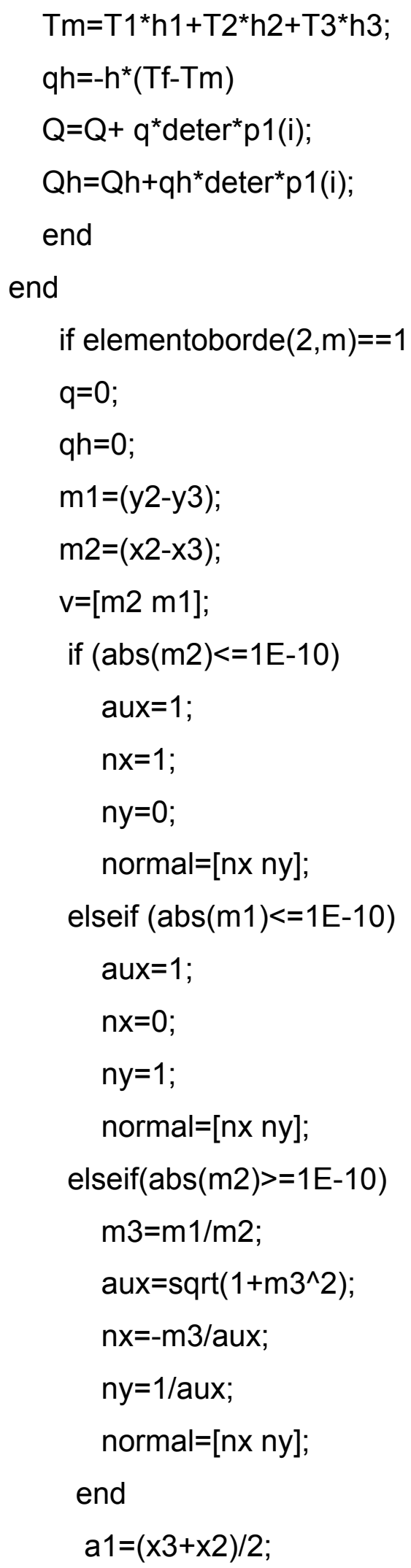




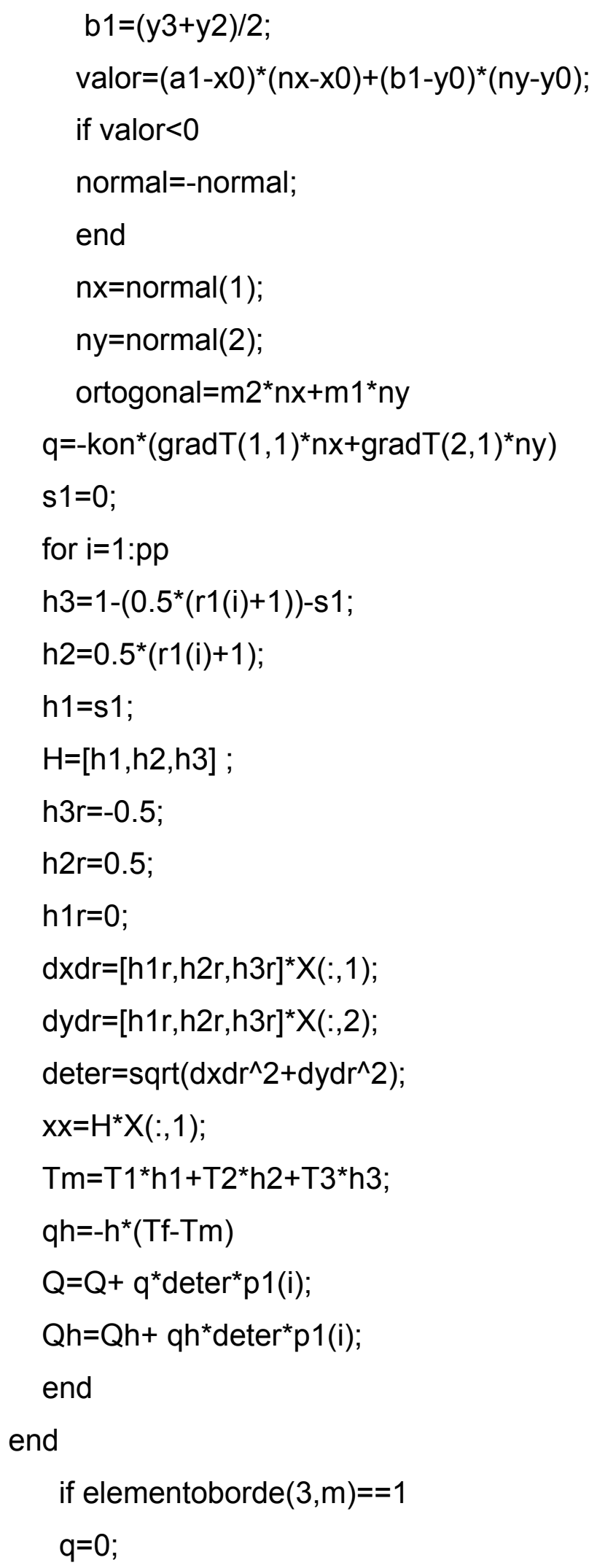




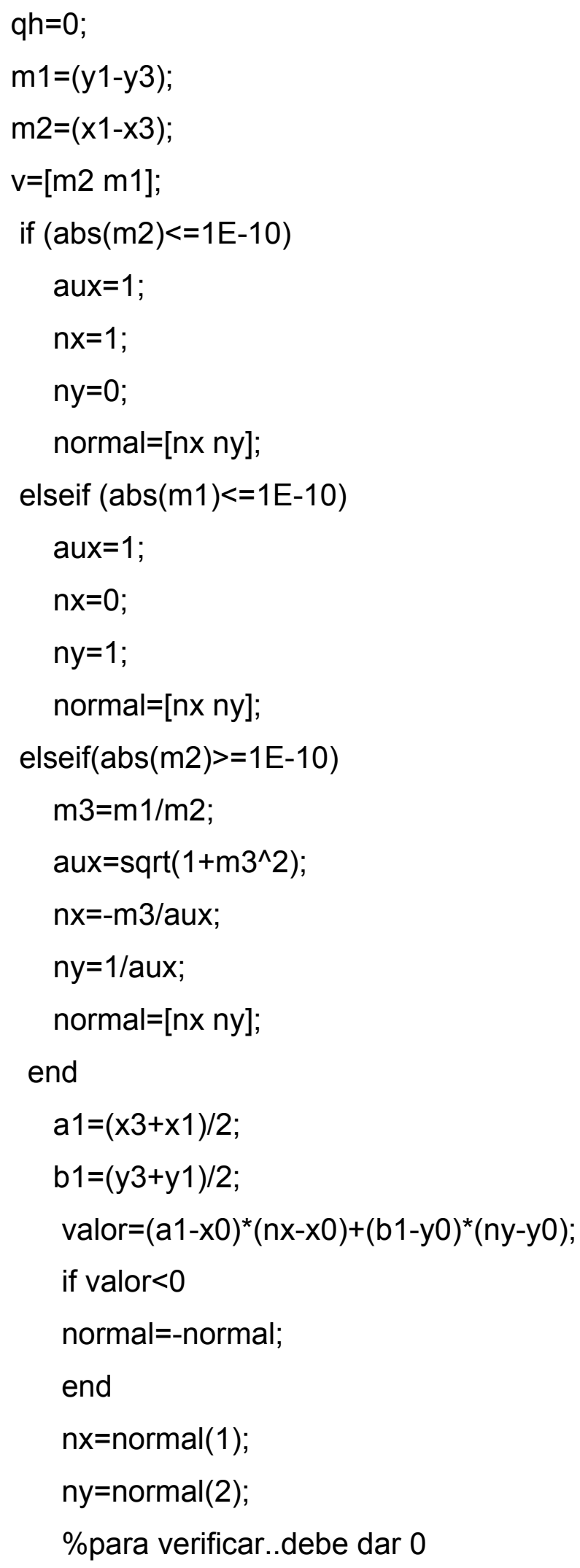




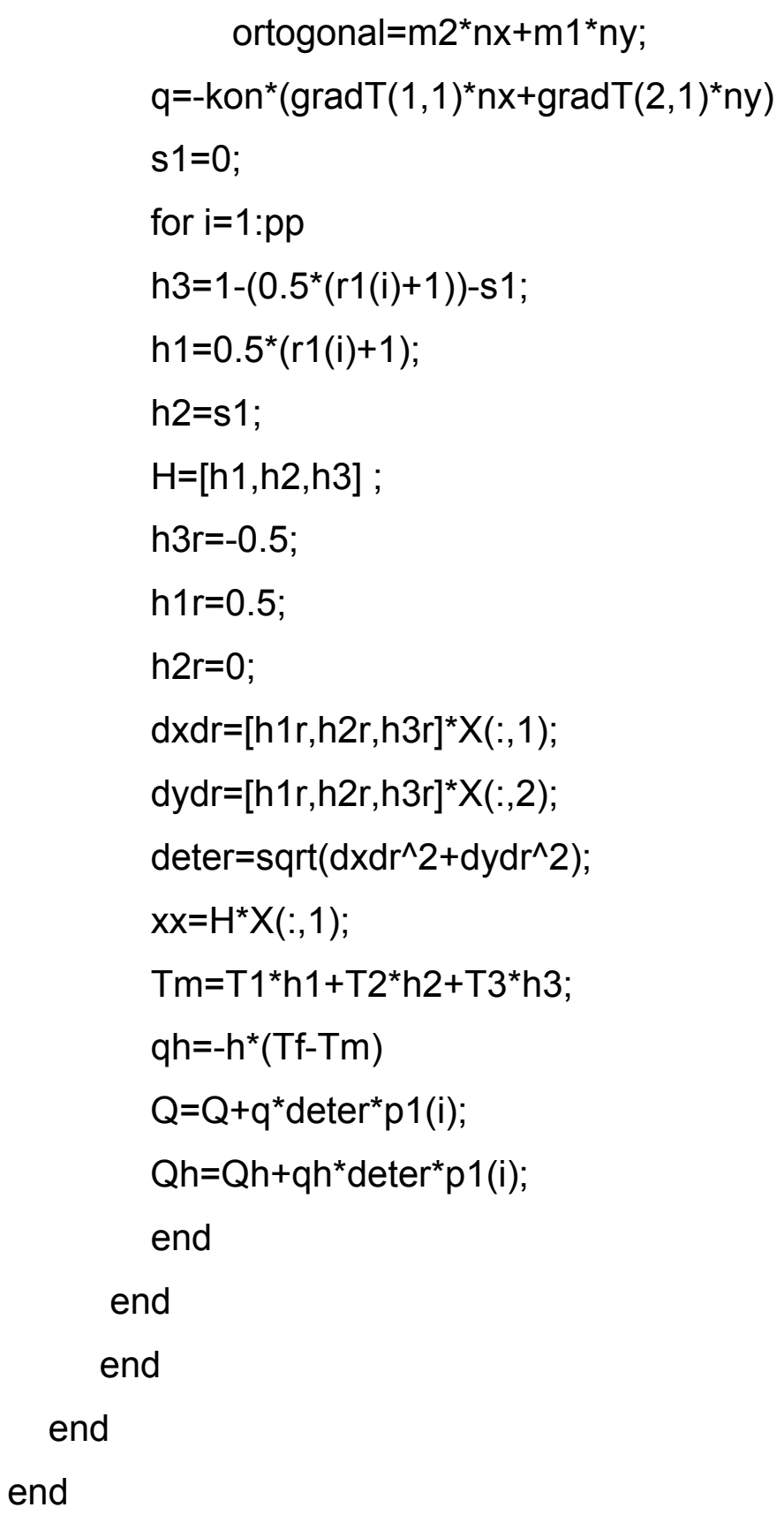

\subsubsection{Temperatura media 2D}

function [Tpromedio,Area]=Tmedia2d(n_elem,nodes, T,coordenadas,pp,rg,sg,p) Tmedia=0;

Área=0;

for $n=1: n \_$elem; 


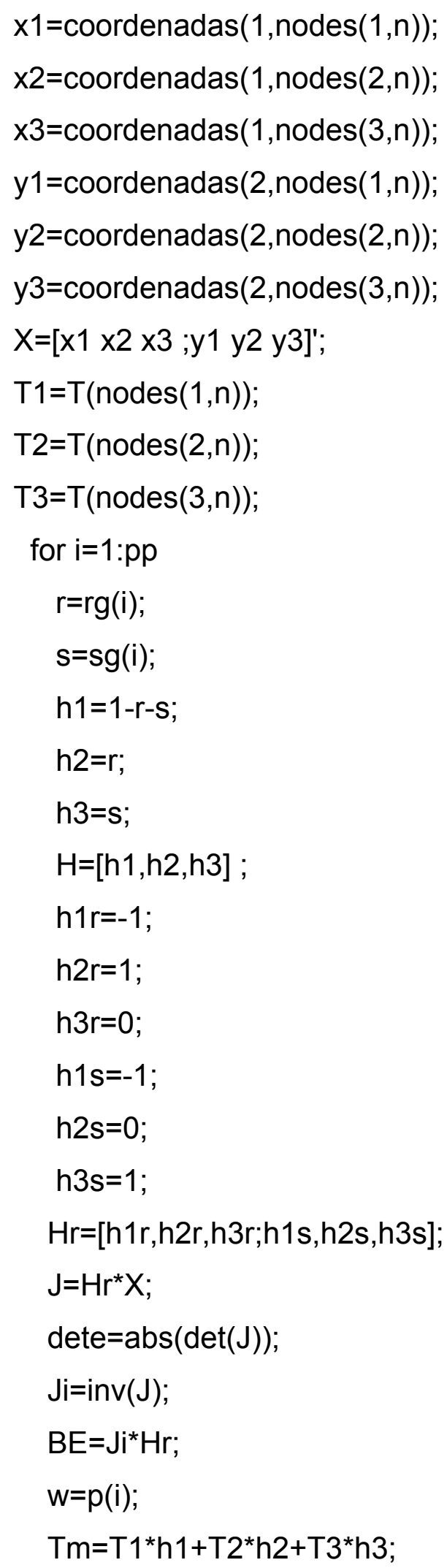



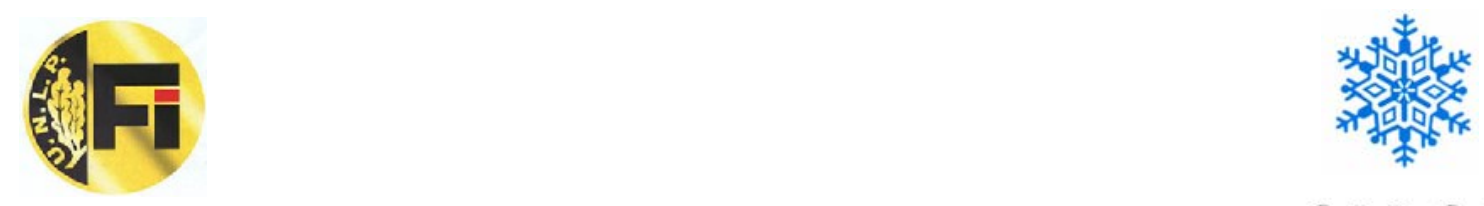

C I D C A

Tmedia $=$ Tmedia $+\mathrm{Tm}^{*}{ }^{*}$ dete $^{*} \mathrm{~W}^{*} 0.5$;

Área=Área $+0.5^{*}$ dete $^{*} \mathrm{w}$;

end

end

Tpromedio=Tmedia/Área;

return 
Capítulo 3 


\section{Aplicación del MEF al procesamiento térmico de alimentos acoplando cinéticas de destrucción microbiana}

\subsection{Introducción}

La presencia del microorganismo Escherichia coli 0157:H7 en productos cárnicos y derivados provoca alteraciones gastrointestinales que pueden ser severas, formando parte de las ETA (enfermedades transmitidas por los alimentos) (Belongia y col., 1991; Doyle, 1991; Riley, 1987). Si bien la bacteria Escherichia coli es frecuentemente encontrada en el intestino de personas sanas, la cepa 0157:H7, en particular, produce toxinas que son la causa principal de los síntomas gastrointestinales pudiendo derivar en Síndrome Urémico Hemolítico (SUH). Este microorganismo es muy peligroso para ciertos grupos de riesgo como los niños, los mayores y las personas que presentan su sistema inmunológico comprometido. Aproximadamente uno de cada diez niños con gastroenteritis por Escherichia coli O157:H7 desarrolla el SUH y esta enfermedad es la primera causa de insuficiencia renal en niños menores de 5 años (Miliwebsky y col., 1999). En la Argentina existen al menos 400 casos al año (Dirección de Epidemiología, 2005), constituyéndose en el país con mayor incidencia mundial. La enfermedad se manifiesta como una diarrea leve, acuosa, que luego se vuelve sanguinolenta y puede desarrollar vómitos, palidez, fiebre, irritabilidad y convulsiones. EI SUH puede causar la muerte o dejar secuelas permanentes como insuficiencia renal crónica, hipertensión arterial y alteraciones neurológicas (Blanco y col., 1995).

Basado en una estimación del año 1999, 73000 casos de infección y 61 muertes ocurren al año en los Estados Unidos (CDC, 2007). El primer brote de diarrea sanguinolenta debido a la presencia de E. coli O157:H7 ocurrió en el año 1982, siendo el origen la ingesta de hamburguesas de carne picada 
contaminada. El microorganismo se podría haber inactivado si se hubiera realizado la completa cocción del producto (temperatura del centro alcanzado los $70^{\circ} \mathrm{C}$ o $68^{\circ} \mathrm{C}$ durante 15 segundos (CFR, 2009; FDA, 2009).

Se han publicado varios trabajos de modelado matemático donde se predijeron perfiles de temperaturas en productos cárneos considerando la inactivación térmica de la E. coli O157:H7 (Pan y col., 2000; Ou y Mittal, 2006). Estos trabajos permitieron establecer condiciones mínimas de tiempos de procesamiento para asegurar la inocuidad de hamburguesas (CFR, 2009; FDA, 2009).

La morcilla es un alimento de consumo habitual en varias partes del mundo como Europa, Asia, y América. "Black Blood Pudding" o morcilla se sirve como parte de un plato de desayuno tradicional en Irlanda, Escocia e Inglaterra (Pearson y Gillet, 1996). Según el Código Alimentario Argentino (2005) "se denomina con el nombre genérico de Morcilla al embutido cocido, elaborado sobre la base de sangre de los animales de consumo permitidos, recogida durante el degüello efectuado en buenas condiciones higiénicas y en recipientes perfectamente lavados, desfibrinada y filtrada, con el agregado o no de tocino, cuero de cerdo picado, sal, especias y otras substancias que hacen al producto y que hayan sido introducidos a presión en fracciones de intestino $u$ otras membranas naturales o artificiales aprobadas a tal fin, aunque en el momento del expendio y/o consumo carezcan del continente". Estos embutidos sufren un proceso de cocción por calor seco (estufas) o en agua con o sin sal, o al vapor por lo que tienen una vida útil mayor. Sin embargo si el proceso de calentamiento no es eficiente esto implicaría un riesgo sanitario potencial para los consumidores. En general en la industria se utilizan grandes recipientes con agua a una temperatura entre $80-90{ }^{\circ} \mathrm{C}$ donde el producto es tratado térmicamente durante 30-40 minutos. El método utilizado para determinar la finalización de la cocción es empírico y se basa en la verificación visual de que la sangre ha coagulado (no se observa líquido) cuando se realiza una incisión en el producto. 
Por otro lado es importante tener en cuenta que en sistemas industriales donde la relación volumen de agua a volumen de embutido es baja, la temperatura del fluido disminuye considerablemente cuando se introducen las piezas cárneas. En algunas industrias de pequeña y mediana escala existen sistemas automáticos de control de temperatura que accionan sobre los equipos de calentamiento (quemadores de gas), sin embargo la inercia térmica del agua impide la instantánea recuperación de la temperatura inicial provocándose el descenso de la temperatura de acuerdo a la relación de carga $(\mathrm{RC}=\mathrm{kg}$ producto/kg agua) utilizada. Este descenso es aún más acentuado cuando se carece de un sistema de control, lo cual es frecuente en industrias de pequeña escala. Como consecuencia se requiere modificar las especificaciones de tiempo-temperatura teniendo en cuenta este descenso de temperatura del agua para lograr un producto inocuo para los consumidores. Es por lo tanto necesaria una estrategia de control térmico para empresas de pequeña y mediana escala en las cuales los operadores juegan un papel importante cuando se quiere implementar estos requerimientos de tiempotemperatura.

En trabajos anteriores realizados en el grupo de investigación, se detectó la presencia de E. coli y E. coli O:157:H7 en morcillas que se obtuvieron de diversos establecimientos de venta al público en la ciudad de La Plata (Oteiza y col., 2006). En este estudio el $76.6 \%$ de las muestras analizadas contenían Escherichia coli y en el 3.3\% de los casos correspondió a Escherichia coli O157:H7. Estos datos permitieron concluir que este producto es de elevado riesgo para el consumidor debido a la presencia de estos patógenos lo cual demuestra que hubo una deficiencia en dos aspectos importantes: la higiene durante la manipulación del alimento y/o el proceso de calentamiento. Cabe resaltar que en la Argentina es costumbre habitual consumir estos embutidos con un tratamiento térmico posterior nulo o insuficiente para la destrucción de los patógenos. El Código Alimentario Argentino no establece las condiciones tiempo-temperatura de calentamiento 
que deben aplicarse a este producto para cumplir con las normas higiénicosanitarias de inocuidad.

La simulación computacional aporta a la industria alimentaria importantes ventajas en cuanto a disminución de costos y tiempos para desarrollo de productos, además de ser una herramienta útil para optimizar el proceso para mejorar la calidad y seguridad microbiológica (Martins, 2006).

Existen en el mercado simuladores comerciales que predicen temperatura en función del tiempo, utilizando el método de los elementos finitos, sin embargo un programa computacional escrito e implementado por el usuario (código abierto) es más fácil de modificar y comprender cuando se busca acoplar subrutinas específicas, que son de interés para simular el problema físico. Este tipo de programas abiertos nos permiten encontrar el punto más frío del alimento (puntos críticos), acoplando en ese punto la cinética de inactivación del microorganismo patógeno, de tal manera que el programa finaliza cuando se alcanza un valor de esterilización dado. Otra ventaja es la posibilidad del acoplamiento de los balances macroscópicos de energía que nos permiten diseñar el algoritmo de control utilizando una temperatura externa variable y considerando el flujo de calor total que ingresa al alimento.

Los objetivos de este Capítulo son:

> Desarrollar un programa computacional propio utilizando el MEF que permita predecir los requerimientos mínimos de tiempo y temperatura para asegurar la destrucción de microorganismos patógenos como la Escherichia coli 0157:H7 mediante la simulación numérica del proceso de calentamiento acoplando la cinética de inactivación térmica de dicho microorganismo.

$>$ Validar el modelo computacional comparando el perfil de temperaturas predichos con las historias térmicas obtenidas mediante experimentos de laboratorio.

$>$ Simular el descenso de temperatura del agua en función de la carga del producto $(\mathrm{RC}=\mathrm{kg}$ producto/kg agua) acoplando el balance microscópico diferencial de energía con el balance macroscópico de energía, validando el 
modelo con mediciones experimentales.

$>$ Establecer requerimientos de tiempos mínimos de proceso para asegurar una reducción de $12_{\log }$ en la población microbiana patógena de E. coli O157:H7 considerando el descenso en la temperatura del agua en función de la RC y de la temperatura inicial del fluido calefactor.

\subsection{Modelo matemático}

La geometría de las morcillas se puede considerar como un sólido de revolución cuya representación gráfica se muestra en la Figura 3-1a). La ecuación diferencial que gobierna la transferencia de energía en coordenadas cilíndricas para un problema axial simétrico se puede expresar de la siguiente manera cuando las propiedades termofísicas son constantes:

$$
\rho \operatorname{Cp} \frac{\partial \mathrm{T}}{\partial \mathrm{t}} r=\frac{\partial}{\partial r}\left(k r \frac{\partial T}{\partial r}\right)+\frac{\partial}{\partial z}\left(k r \frac{\partial T}{\partial z}\right)
$$

Esta ecuación es válida en el dominio $\Omega$ siendo $T$ la temperatura, $t$ el tiempo del proceso de proceso, $\rho$ la densidad, $\mathrm{k}$ la conductividad térmica, $\mathrm{Cp}$ el calor específico del material, $r$ y z las coordenadas geométricas.

Las condiciones iniciales y de contorno son las siguientes:

$$
\begin{array}{lcc}
\mathrm{T}=\mathrm{T}_{0} & \mathrm{t}=0 & \text { en } \Omega \\
\left(\frac{\partial \mathrm{T}}{\partial \mathbf{Z}} \cdot \mathrm{nz}+\frac{\partial \mathrm{T}}{\partial \mathrm{r}} \cdot \mathrm{nr}\right) \mathrm{k}=\mathrm{h}\left(\mathrm{T}_{\text {ext }}-\mathrm{T}\right) & \mathrm{t} \geq 0 & \text { en } \delta \Omega_{1} \\
\left(\frac{\partial \mathrm{T}}{\partial \mathbf{Z}} \cdot \mathrm{nz}+\frac{\partial \mathrm{T}}{\partial \mathrm{r}} \cdot \mathrm{nr}\right) \mathrm{k}=0 & \mathrm{t} \geq 0 & \text { en } \delta \Omega_{3}
\end{array}
$$


donde $\partial \Omega_{1}$ corresponde a la interfase convectiva y $\partial \Omega_{3}$ al eje de simetría, respectivamente, $\mathrm{nz}$ y $\mathrm{nr}$ son las componentes del vector normal unitario exterior a la curva que forma el contorno del alimento, $\mathrm{T}_{\text {ext }}$ es la temperatura del medio calefactor, $\mathrm{T}_{0}$ la temperatura inicial y $\mathrm{h}$ el coeficiente de transferencia de calor en la interfase.

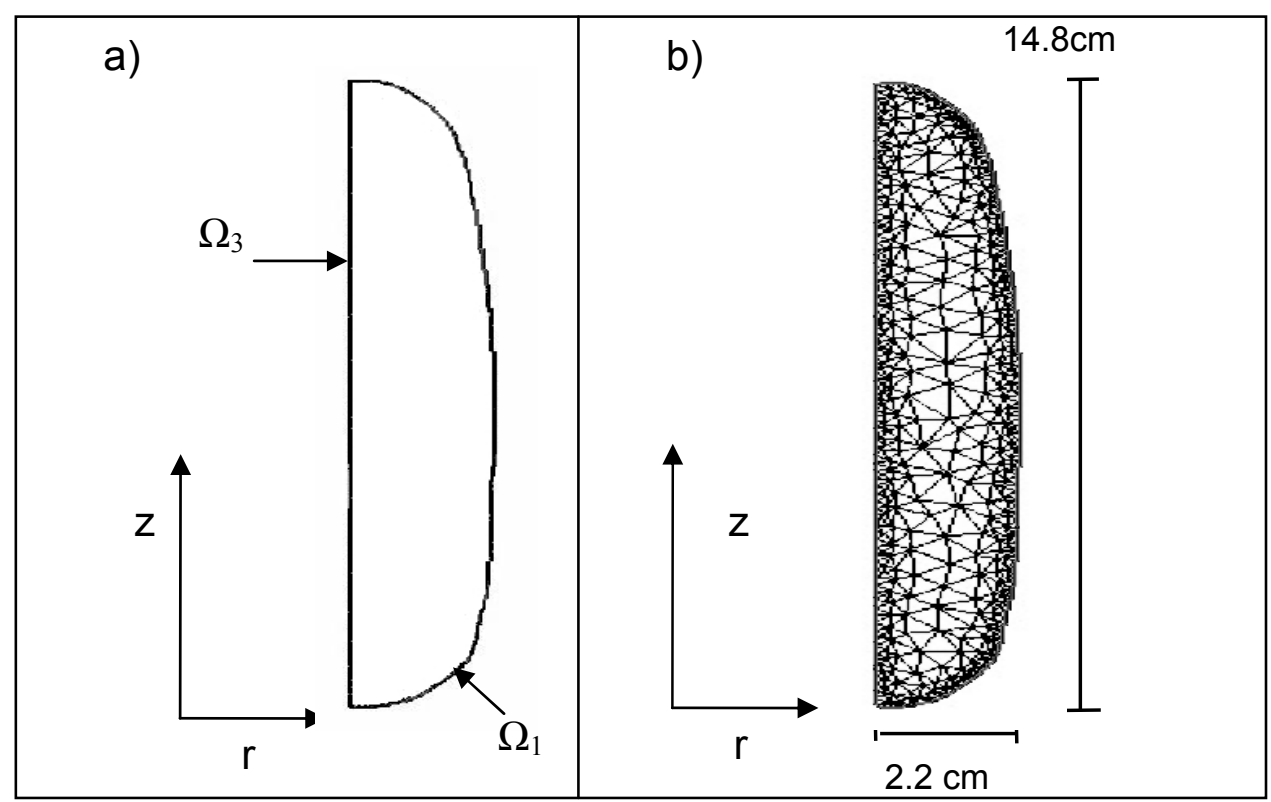

Fig. 3-1 a) Superficie de revolución cuyo contorno representa la geometría del producto. b) Discretización en elementos triangulares de la geometría

Para discretizar el dominio de geometría irregular, se utilizaron imágenes digitales del producto a simular obteniendo la curva que definía el contorno del producto, suponiendo que el alimento presentaba un eje de simetría en $r=0$. Luego, utilizando el generador de malla COMSOL se obtuvo la grilla de coordenadas espaciales, la estructura de la malla y la relación entre los elementos y nodos del dominio. Esta información se alimentó al programa principal, desarrollado en el presente trabajo de Tesis, para realizar los cálculos. La grilla utilizada en el programa desarrollado para la simulación numérica del proceso de calentamiento está compuesta por 748 nodos totales y 1324 elementos triangulares (Figura 3-1 b). Se tomaron suficiente cantidad de nodos y elementos como para maximizar la precisión de la solución numérica y mantener el tiempo de cálculo dentro de un rango aceptable. 


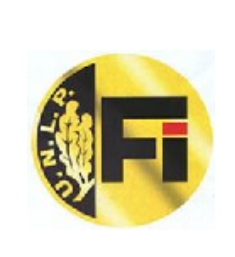

C I D C A

La $\mathrm{T}_{\text {ext }}$ representa una variable importante, ya que puede descender notoriamente cuando las morcillas se sumergen en el fluido calefactor. La $T_{\text {ext }}$ depende de la cantidad de producto que está siendo procesado, el volumen de agua del recipiente, y del calor efectivo entregado por los equipos de calentamiento (quemadores).

La formulación variacional de las ecuaciones 3.1 a 3.4 requiere del mismo procedimiento que el expuesto en el Capítulo 2, sin embargo presenta algunas diferencias debido a la transformación de variables en coordenadas cilíndricas. La distribución de temperaturas $\left(\mathrm{T}^{\mathrm{e}}\right)$ en cualquier punto dentro de un elemento del dominio se aproxima usando las el vector que contiene las funciones de interpolación $(H)$ y las temperaturas nodales $(T 1, T 2, T 3)$ en el elemento correspondiente. La temperatura fue representada utilizando un espacio de elementos finitos $V_{h}$ con las funciones de interpolación $H$. La ecuación resultante luego de aplicar el método de Galerkin y el Teorema de la Divergencia es (Bathe, 1996; Zienkiewicz y Taylor, 1994 a):

$\mathrm{CG} \cdot \dot{\mathrm{T}}+\mathrm{KG} \cdot \mathrm{T}=\mathrm{FG}$

donde $C G=\sum_{e=1}^{n} \int_{\Omega_{e}}\left(H^{\top} r \rho C p H\right) d \Omega$ e es la matriz global de capacitancia

$\mathrm{KG}=\sum_{\mathrm{e}=1}^{\mathrm{n}} \int_{\Omega_{\mathrm{e}}}\left(\mathrm{B}^{\top} \mathrm{rkB}\right) \mathrm{d} \Omega_{\mathrm{e}}+\sum_{\mathrm{e}_{1}=1}^{\mathrm{n}} \int_{\delta \Omega_{\mathrm{e}_{1}}}\left(\mathrm{H}^{\top} \mathrm{rhH}\right) \mathrm{d} \delta \Omega_{\mathrm{e} 1}$ es la matriz global de conductancia

$\mathrm{FG}=\sum_{\mathrm{e}_{1}=1}^{\mathrm{n}} \int_{\delta \Omega \mathrm{e}_{1}}\left(\mathrm{H}^{\top} \mathrm{rhH} \mathrm{T}_{\text {ext }}\right) \mathrm{d} \delta \Omega_{\mathrm{e} 1}$ es el vector de fuerzas globales donde $\mathrm{T}$ es el vector que representa los valores de temperatura en los nodos, $\dot{\mathrm{T}}$ representa la $\frac{\partial \mathrm{T}}{\partial \mathrm{t}}$ (donde $\mathrm{T}$ es el valor de temperatura en el nodo). 
Este problema semi-discreto (Ec. 3.5) es un sistema de ecuaciones diferenciales ordinarias.

La Ec. 3.5 se discretizó en el tiempo utilizando el método a (Pham, 2006). En el programa computacional se utilizó $\alpha=0.5$ correspondiendo al método de discretización en el tiempo de Crank-Nicolson, el cual es incondicionalmente estable (Zienkiewicz y Taylor, 1994 b).

$$
\left(\Delta t^{-1} C G+\alpha K G\right) T^{t+\Delta t}=F G+\left(\Delta t^{-1} C G-\alpha K G\right) T^{t}
$$

El programa computacional fue implementado en Matlab 6.5 y permite calcular los cambios de temperatura durante el calentamiento en cada punto del dominio, el flujo de calor total que ingresa al alimento, la temperatura promedio del alimento, etc. Para cada una de estas subrutinas se requiere alimentar la variable independiente (temperatura) en cada lapso o incremento en el tiempo.

\subsubsection{Balance macroscópico de energía}

En las plantas industriales los embutidos son procesados térmicamente en sistemas batch. Los embutidos son introducidos en recipientes con agua a una temperatura inferior a los $100^{\circ} \mathrm{C}$ para su cocción, evitando así la ruptura de los productos. El agua calentada previamente mediante quemadores a gas, presenta un abrupto descenso de temperatura cuando se sumergen las piezas cárneas. La recuperación de la temperatura del fluido a su valor inicial depende fundamentalmente de dos parámetros:

1. la relación de carga $(\mathrm{RC}=\mathrm{kg}$ producto $/ \mathrm{kg}$ agua)

2. el calor efectivo entregado por los quemadores.

Cabe resaltar que en las plantas de procesamiento más equipadas, que presentan un control de temperatura que acciona sobre los quemadores, la recuperación de la temperatura del fluido no es instantánea, debido a la inercia 


\section{(F)}

C I D C A

térmica del agua.

Para describir las condiciones reales de procesamiento es necesario resolver el balance macroscópico de energía acoplado con el balance microscópico diferencial de energía el producto individual. El programa computacional calcula a cada incremento del tiempo el flujo de calor total ingresado integrando numéricamente el flujo en toda el área expuesta con el fluido $\left(\int-\mathrm{k}(\nabla \mathrm{T} \cdot \mathrm{n}) \mathrm{dA}\right)$; esta cantidad de energía se multiplicó por el número de embutidos procesados en cada batch para calcular $Q_{\text {producto. La energía en }}$ forma de calor se transfirió desde el quemador al fluido y desde el fluido a los embutidos. Se consideró la pérdida de calor debido a evaporación y a través de las paredes del recipiente $\left(Q_{\text {perdido }}\right)$.

Por ende el balance macroscópico acoplado al balance microscópico es el siguiente:

$Q_{\text {quemador }}=Q_{w}+Q_{\text {producto }}+Q_{\text {perdido }}$

donde el calor entregado desde el quemador al agua $\left(Q_{w}\right)$ se puede expresar como:

$Q_{w} \Delta t=m_{w} C p_{w}\left(T_{e x t, j+1}-T_{e x t, j}\right)$

$\mathrm{T}_{\text {ext,j }}$ y $\mathrm{T}_{\text {ext,j+1 }}$ representan la temperatura del agua a un tiempo j y j +1 respectivamente, $\mathrm{m}_{\mathrm{w}}$ es la masa de agua en el recipiente, y $\Delta$ t es el intervalo de tiempo. Al comienzo del proceso $T_{\text {ext,j }}=T_{\text {ext, } 0}$ el cual corresponde a la temperatura inicial del fluido, $\mathrm{T}_{\text {ext,j+1 }}$ es la nueva temperatura del fluido calefactor. Se generó un algoritmo considerando las ecuaciones 3.7 y 3.8 para implementar la subrutina cuya variable de entrada era $T_{\text {ext,j }}$ y la variable de salida $T_{\text {ext,j+1 }}$, la cual era utilizada para calcular en el siguiente incremento en el tiempo en el programa principal. El paso en el tiempo utilizado para el cálculo 
de $T_{\text {ext,j+1 }}$ fue de $5 \mathrm{~s}$.

La integración del flujo de calor ingresado al alimento durante el calentamiento permite calcular la historia térmica del fluido calefactor, $T_{\text {ext }}$, en función de la relación de carga (RC).

\subsubsection{Cinética de inactivación microbiana}

Teniendo en cuenta que la disminución de una población microbiana generalmente sigue una forma exponencial, el cambio producido en la población debería seguir una cinética de primer orden. Entonces:

$$
\frac{\mathrm{dN}}{\mathrm{dt}}=-\mathrm{kr} \cdot \mathrm{N}
$$

donde $\mathrm{kr}$ es la constante cinética de muerte térmica $\left(\mathrm{min}^{-1}\right)$, función de la temperatura, $\mathrm{t}$ es el tiempo (min) y $\mathrm{N}$ es el número de unidades formadoras de colonia por gramo (UFC/gr). Integrando la función desde una población inicial $\mathrm{N}_{0}$ (UFC/gr) hasta una población microbiana final $(\mathrm{N})$ se obtiene:

$2.303 \cdot \log \left(\frac{N}{N_{0}}\right)=-k r \cdot t$

$D_{\text {ref }}$ es el tiempo de reducción decimal $(\min )$ que representa el tiempo requerido para reducir un $90 \%$ la población microbiana a una temperatura determinada. Si se grafica en una escala semilogarítmica la disminución en la población microbiana versus tiempo se puede observar que el parámetro $D_{\text {ref }}$ representa el tiempo necesario para lograr una disminución de un ciclo logarítmico en la población microbiana (Fig. 3-2). 
C I D C A

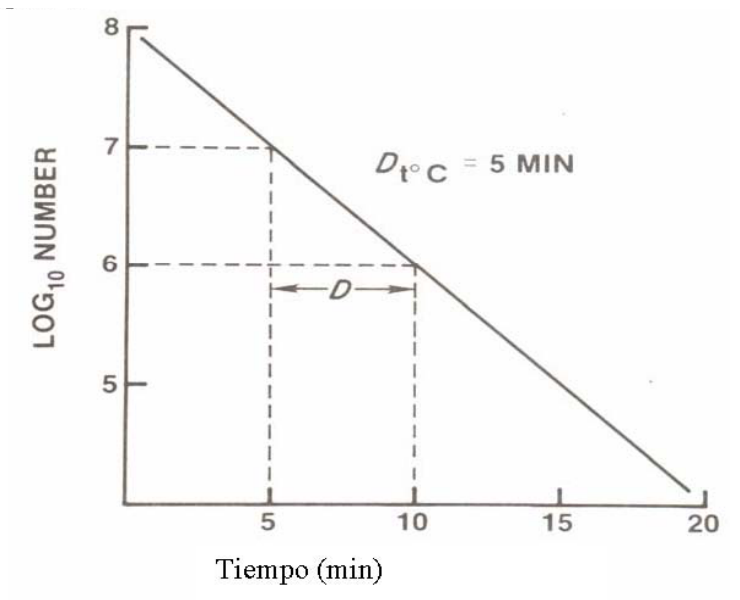

Fig. 3-2 Gráfico que ilustra el tiempo de reducción decimal ( $\left.D_{\text {ref }}\right)$

La constante de resistencia térmica $Z\left({ }^{\circ} \mathrm{C}\right)$ es el incremento de temperatura necesario para disminuir $D_{\text {ref }}$ en un $90 \%$ (Fig. 3-3).

$$
Z=\frac{T-T_{\text {ref }}}{\log \left(D_{\text {ref }} / D\right)}
$$

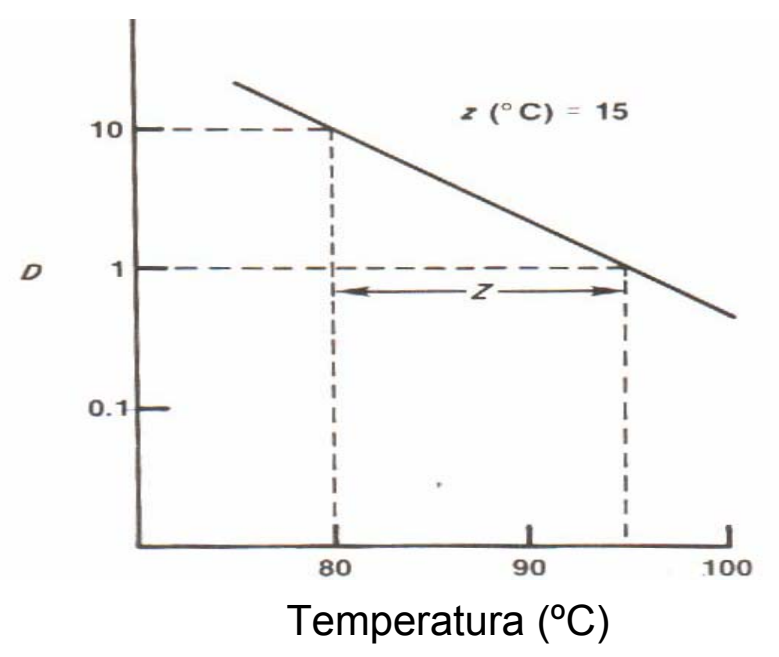

Fig. 3-3 Gráfico que ilustra la constante de resistencia térmica (Z)

El tiempo de muerte térmica, $F$, es el tiempo necesario para causar una 
determinada reducción en la población de microorganismos o esporas a una temperatura de referencia. Se lo define en general como un múltiplo del valor de $D_{\text {ref. }}$ Por ejemplo si tomamos un valor $\alpha$ :

$$
F=\log \left(\frac{N_{o}}{N}\right) \cdot D_{\text {ref }}
$$

En general el valor típico de número de reducción de ciclos logarítmicos $\left(\log \left(N_{\circ} / N\right)=12\right)$ se adopta para procesos de esterilización, lo que equivale a reducir 12 ciclos logarítmicos la población microbiana.

A partir de la Ec. 3-9 y sabiendo que $\mathrm{kr}=2.303 / \mathrm{D}$ a una temperatura $\mathrm{T}$ se deduce que:

$$
\int_{N_{0}}^{N} \frac{d N}{N}=\int_{0}^{t}-k r \cdot d t=\int_{0}^{t}-\frac{2.303}{D} \cdot d t
$$

Utilizando un cambio de base de logaritmo neperiano a decimal y la Ec. 3-11 resulta que el cociente del $F$ del proceso $\left(F_{\text {proceso }}\right)$ real y el $D_{\text {ref }}$ es:

$$
\frac{F_{\text {proceso }}}{D_{\text {ref }}}=\log \left(\frac{N_{0}}{N}\right)=\int_{0}^{t} \frac{10^{\frac{\left(T-T_{\text {ref }}\right)}{Z}}}{D_{\text {ref }}} \cdot d t
$$

A partir de los parámetros cinéticos del microorganismo patógeno Escherichia coli O157:H7, la carga inicial $\mathrm{N}_{0}$, y la historia térmica en el punto más frío del alimento $(T)$ se puede integrar numéricamente la Ec. 3-14 y obtener los valores de $\log \left(\mathrm{N}_{0} / \mathrm{N}\right)$ a cada tiempo adoptando intervalos pequeños de tiempo:

$$
\log \left(\frac{N_{0}}{N}\right)=\sum_{n=1}^{m} 10^{\frac{\left(T_{c}(n)-T_{\text {ref }}\right)}{z}} \cdot \frac{\left(t_{n+1}-t_{n}\right)}{D_{\text {ref }}}
$$


donde $T_{c}(n)$ es un vector de temperaturas en el punto frío, $m$ es el número de intervalos de tiempo, y $\Delta t=t_{n+1}-t_{n}$ es el incremento en el tiempo.

En estudios previos (Oteiza y col., 2003) se determinaron los parámetros de letalidad para la inactivación térmica de la $E$. coli 0157:H7 en morcillas; $\mathrm{D}_{\text {ref }}$ (2.74 $\mathrm{min}), \mathrm{T}_{\text {ref }}\left(57^{\circ} \mathrm{C}\right)$, y $\mathrm{Z}\left(7.44^{\circ} \mathrm{C}\right)$. Se utilizó un valor de inactivación $(\mathrm{VI}=$ $\left.\log \left(\mathrm{N}_{0} / \mathrm{N}\right)\right)$ de $12_{\log }$ para determinar los tiempos de procesamiento, dado que estos productos se consumen frecuentemente sin un posterior calentamiento. Es importante tener en cuenta que la presencia de 10 organismos de E. coli 0157:H7 son suficientes para causar enfermedad en humanos (FDA, 2008).

Suponiendo un valor inicial de $\mathrm{N}_{0}$ de $10^{4} \mathrm{UFC} / \mathrm{g}$ y un peso promedio de morcilla de $100 \mathrm{~g}$, la población total inicial de patógenos en una morcilla sería de $10^{6}$ UFC/embutido. Luego del tratamiento térmico en el que se alcanza un $\mathrm{VI}=12_{\log }$ y considerando que la probabilidad de supervivencia microbiana sigue una distribución de Poisson, la probabilidad de no encontrar ningún UFC/embutido (o menos de 1 UFC/embutido) es $P(x<1)=\exp \left(10^{-6}\right)=0.999999$. Por ende la probabilidad de encontrara al menos 1 UFC/embutido, $P(x>1)=$ 0.000001 , es decir 1 UFC cada $1.10^{6}$ embutidos tratados, que puede suponerse una probabilidad suficientemente baja como para asumir una esterilización eficiente.

El programa computacional predice los tiempos mínimos de procesamiento para alcanzar un $\mathrm{VI}=12_{\log }$ incorporando la cinética de inactivación en el modelo numérico.

Se encontraron los requerimientos adecuados de tiempo de proceso para que el sistema alcance un $\mathrm{VI}=12_{\log }$ considerando el descenso en la temperatura del fluido calefactor y acoplando la cinética de inactivación microbiana y el balance macroscópico de energía. En el siguiente esquema del diagrama de flujo se ilustra los pasos seguidos por el programa codificado en Matlab (Fig. 3-4). 


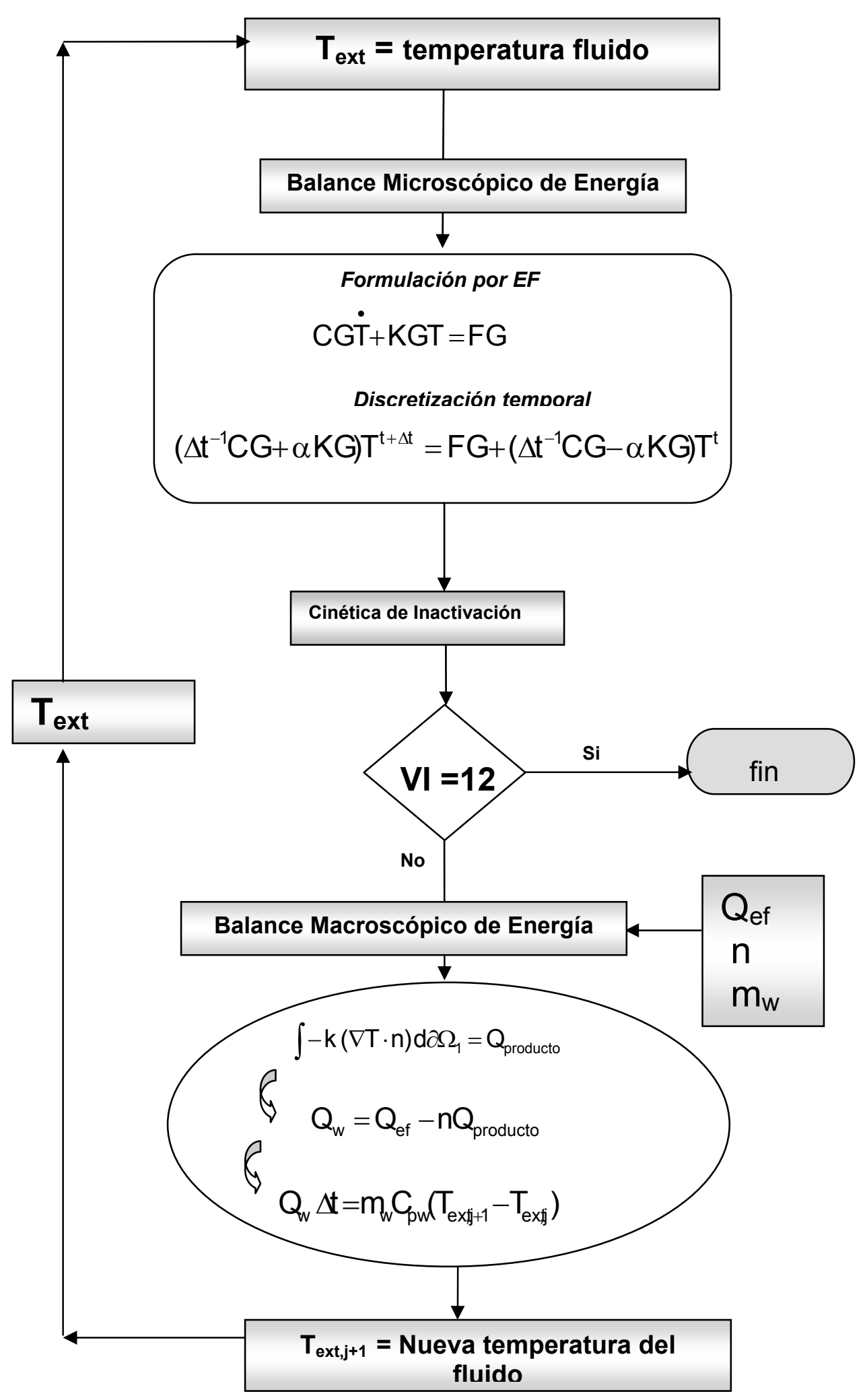

Fig. 3-4 Esquema del diagrama de flujo del programa computacional con el acoplamiento de la letalidad microbiana y el balance macroscópico de energía 


\subsection{Materiales y métodos}

\subsubsection{Procedimiento para la validación experimental del balance microscópico de energía durante el calentamiento considerando temperatura de fluido constante}

Se realizaron experiencias de calentamiento sumergiendo unidades de embutido (aproximadamente de $2 \mathrm{~cm}$ de radio y $14 \mathrm{~cm}$ de largo) en un baño termostático con agitación interna (Haake F3, Alemania). Se registraba la temperatura en distintos puntos del producto y del fluido calefactor, mediante termocuplas tipo T (Cobre-Constantán); las termocuplas se conectaron a adquisidores (Testo 175, Testo AG, Alemania). Las experiencias se daban por finalizadas cuando se alcanzaba el equilibrio térmico con la temperatura del baño. Luego se localizaba la posición de las termocuplas cortando el alimento en finas secciones trasversales. Para evitar el cambio en la posición de las termocuplas al seccionar el alimento se enfriaron las muestras para que presentaran mayor rigidez. Previo al calentamiento se pesaban y se fotografiaban las muestras (Kodak Easy Share, NY).

El peso promedio de las unidades fue $150 \mathrm{~g}$ y la composición química según el proveedor, sin la piel, fue: $15.58 \%$ proteínas, $11.68 \%$ grasa, $1.97 \%$ carbohidratos, $68.58 \%$ agua, y $2.19 \%$ cenizas.

Se realizaron diez experimentos utilizando distintas temperaturas de agua ( $T_{\text {ext }}$ ); en estos experimentos la $T_{\text {ext }}$ estuvo comprendida en un rango de 60 a $90{ }^{\circ} \mathrm{C}(66.6,76.3,78,78.2,79,82,85.5,86,88$, y 90). Las propiedades termofísicas del producto que se alimentaron al programa principal son: $\rho=1000 \mathrm{~kg} / \mathrm{m}^{3}, \mathrm{k}=0.53 \mathrm{~W} / \mathrm{m}^{\circ} \mathrm{C}, \mathrm{y} \mathrm{Cp}=3230 \mathrm{~J} / \mathrm{kg}{ }^{\circ} \mathrm{C}$ (Adam y col., 1997). 


\subsubsection{Determinación del coeficiente de transferencia de calórica en el baño termostático}

Para la estimación del coeficiente de transferencia de calor del sistema, se utilizó una esfera de aluminio cuyas propiedades y dimensiones eran conocidas $\left(R=0.0152 \mathrm{~m}, \mathrm{k}=178.35 \mathrm{~W} / \mathrm{m}^{\circ} \mathrm{C}, \rho=2700 \mathrm{~kg} / \mathrm{m} 3, \mathrm{Cp}=908.53\right.$ $\mathrm{J} / \mathrm{kg}{ }^{\circ} \mathrm{C}$ ). La esfera se sumergía en el baño termostático utilizado para el calentamiento de los embutidos y se registraba a través de un sistema de adquisición (TESTO175, TESTO AG, Alemania), la temperatura en el centro y superficie en función del tiempo.

Comparando las temperaturas experimentales con las predichas por la solución analítica proponiendo distintos coeficientes de transferencia calórica (h), se determinó el coeficiente óptimo que minimizaba la varianza:

$$
\sigma^{2}=\frac{\sum\left(\mathrm{T}_{\text {exp }}-\mathrm{T}_{\text {predicha }}\right)^{2}}{\mathrm{M}-1}
$$

donde $\mathrm{M}$ es el número de mediciones experimentales.

\subsubsection{Determinación del calor efectivo del quemador}

A lo efectos de tratar de reproducir las condiciones de procesamiento térmico de los embutidos en condiciones industriales se utilizó un recipiente con agua, de $10 \mathrm{~L}$ similar al de una planta elaboradora de pequeña escala, calefaccionado mediante un quemador a gas.

Se realizaron experimentos para determinar el calor efectivo entregado por dicho quemador.

El agua dentro del recipiente se calentó utilizando un quemador industrial de escala piloto hasta alcanzar una temperatura máxima de $90^{\circ} \mathrm{C}$. El aumento de temperatura en función del tiempo se almacenó en adquisidores utilizando cuatro termocuplas localizadas en distintas posiciones dentro del 
recipiente. Utilizando esta información se calculó el calor efectivo $\left(Q_{\mathrm{ef}}\right)$ del quemador al fluido calefaccionante con la siguiente ecuación (3.11), obteniendo el valor de $1450 \mathrm{~W}$.

$$
Q_{\text {ef }}=Q_{\text {quemador }}-Q_{\text {perdido }}
$$

\subsubsection{Experimentos para validar el descenso de temperatura del fluido de calentamiento en función de la relación de carga $(\mathrm{RC})$ modelado de acuerdo 3.2.1}

Se realizaron experimentos de calentamiento sumergiendo diferente número de embutidos en el recipiente descripto en la Sección anterior (3.3.3) para determinar el descenso de temperatura en el fluido como función de la carga (kg de producto).

Se utilizaron cuatro termocuplas ubicadas en distintos puntos en el fluido calefaccionante con el objeto de registrar el descenso de temperatura en función del tiempo. Se realizaron los experimentos con y sin agitación del fluido; en el segundo caso se utilizó un agitador mecánico (Euro ST-PB 79219, Alemania) a una velocidad de $100 \mathrm{rpm}$.

Para estimar el coeficiente de transferencia calórica (h) en el recipiente de calentamiento, con y sin agitación se utilizó el mismo procedimiento que el descripto en la Sección 3.3.2.

Estos experimentos permitieron validar las predicciones del modelo numérico.

\subsubsection{Aplicaciones del modelo numérico desarrollado para predecir tiempos de procesamiento variando condiciones operativas}

Se realizaron simulaciones numéricas en el programa computacional variando la temperatura inicial del fluido calefactor $\left(T_{\text {ext,0 }}=75-95^{\circ} \mathrm{C}\right)$, el 
coeficiente de transferencia en la interfase ( $h=800-1150 \mathrm{~W} / \mathrm{m}^{2}{ }^{\circ} \mathrm{C}$ ), y la relación de carga $(\mathrm{RC}=0.15-0.75 \mathrm{~kg}$ producto/ $\mathrm{kg}$ agua). El programa numérico para las distintas condiciones operativas analizadas ( $h, T_{\text {ext,0, }}, R C$ ) finalizaba al alcanzar un valor de inactivación de $12_{\log }$ permitiendo calcular el tiempo mínimo de proceso.

\subsection{Resultados y discusión}

\subsubsection{Coeficiente de transferencia calórica en la interfase.}

El modelo numérico se validó en primera instancia considerando una temperatura del fluido calefactor constante para lo cual se estimó previamente el coeficiente de transferencia calórica en la interfase utilizando el baño termostático con agitación descripto en 3.3.2. El coeficiente de transferencia calórica en la interfase que permitía ajustar adecuadamente las temperaturas experimentales con las predichas, fue de $\mathrm{h}=1615 \mathrm{~W} / \mathrm{m}^{2}{ }^{\circ} \mathrm{C}$.

Luego se utilizó el modelo para simular las condiciones industriales realizando el procesamiento térmico en un recipiente con agua, donde se produce un descenso en la temperatura del fluido calefactor debido a la introducción de la carga fría. El coeficiente de transferencia de calor del sistema, determinado a través del calentamiento de la esfera aluminio $(h)$, con y sin agitación, fue de 1105 y $828 \mathrm{~W} / \mathrm{m}^{20} \mathrm{C}$ respectivamente.

En ambos casos la formación de burbujas incipientes en la parte inferior del recipiente en contacto con el quemador (nucleate pool boiling) condujo a la generación de altas velocidades de circulación de líquido y por ende a valores altos del coeficiente de transferencia calórico.

Además los valores de $\mathrm{h}$ fueron calculados utilizando una ecuación que permite evaluar los valores de h para placas horizontales en burbujeo nucleado (Geankoplis, 1993): 
donde $\Delta \mathrm{T}=\mathrm{TV}-\mathrm{Tbw}\left({ }^{\circ} \mathrm{C}\right)$, con $\mathrm{Tv}=$ Temperatura en la superficie inferior del recipiente, Tbw $=$ temperatura de equilibrio líquido-vapor a $1 \mathrm{~atm}, 100{ }^{\circ} \mathrm{C}$. Los valores de $\mathrm{h}$ utilizando los datos de $\Delta \mathrm{T}$ (rango entre $3-6^{\circ} \mathrm{C}$ ) en la ecuación 3.18 concuerdan satisfactoriamente con los valores experimentales de $h$.

\subsubsection{Validación del proceso de calentamiento del producto cárnico utilizando una temperatura de fluido constante.}

En la Figuras 3-5 a) y b) se puede observar la excelente concordancia entre los resultados predichos por el programa de simulación y las temperaturas experimentales obtenidas durante el calentamiento de los productos cárneos. La experiencia mostrada a modo de ejemplo correspondió a una temperatura del medio calefactor de $66.6^{\circ} \mathrm{C}$. El error porcentual promedio entre las temperaturas experimentales y predichas fue de $3.2 \%$. La posición de cada termocupla se corresponde con los siguientes nodos en la grilla utilizada para discretizar el dominio: T1 (nodo 171), T2 (nodo 31) y T3 (nodo 461).

El tiempo máximo de cálculo computacional, utilizando la malla descripta en la sección 2.2, fue de 8 minutos. Se calculó el error relativo porcentual $\left(e_{r}(\%)=\left(\left(T_{\text {exp }}-T_{\text {pred }}\right) / T_{\text {exp }}\right) \cdot 100 \%\right)$, donde $T_{\exp }$ es la temperatura experimental, y $T_{\text {pred }}$ es la temperatura predicha, ambas a un tiempo $t$. Teniendo en cuenta todos los experimentos realizados el $e_{r} \%$ máximo fue de $1.5 \%$, por lo tanto se puede concluir que el código desarrollado reproduce con buena precisión los datos experimentales. 
C I D C A
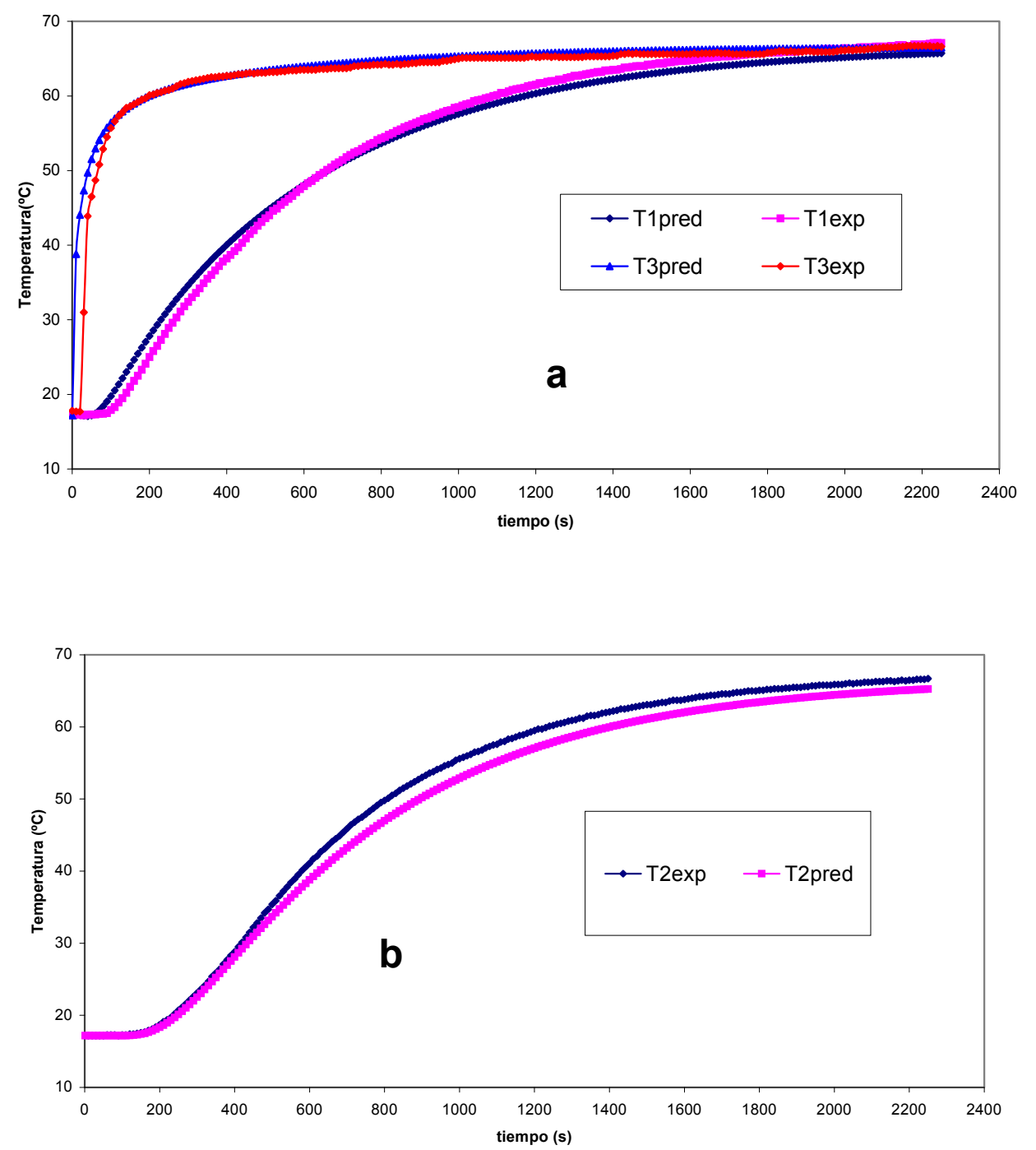

Fig. 3-5 a) Comparación entre los resultados numéricos $\left(T_{\text {pred }}\right)$ y las historias térmicas experimentales $\left(T_{\text {exp }}\right)$ correspondientes al calentamiento de una pieza de embutido. (Temperatura del medio calefactor $=66.6^{\circ} \mathrm{C}$ ). Posición de termocupla $\mathrm{T} 1$ : radial: $1.2 \mathrm{~cm}$, axial: $7.35 \mathrm{~cm}$. Posición de termocupla T3: radial:2.21 cm, axial: $6.97 \mathrm{~cm}$ b) Posición de termocupla T2: radial: $0.0 \mathrm{~cm}$, axial: $7.0 \mathrm{~cm}$ 


\subsubsection{Validación del balance macroscópico de energía acoplado al balance microscópico para evaluar el descenso de temperatura del fluido calefactor}

Los experimentos descriptos en la Sección 3.3.4 mostraron que la temperatura del agua descendía considerablemente cuando se sumergía la carga de embutidos fría. Este comportamiento se observó para las condiciones experimentales de convección forzada y de convección natural (sin agitación), de acuerdo a lo que se observa en las Fig. 3-6 a) y b).

En dichas figuras se muestran a modo de ejemplo las temperaturas experimentales y simuladas durante el calentamiento para sistemas con y sin agitación respectivamente. Las simulaciones numéricas permitieron predecir con exactitud el descenso de temperatura del agua para distintas relaciones de carga y temperaturas iniciales de fluido calefactor. El error porcentual promedio entre las temperaturas predichas y experimentales fue de $2.1 \%$ y $2.2 \%$ para condiciones de agitación y sin agitación respectivamente.

Una vez validado el modelo numérico que simula el acoplamiento de balances energéticos, se analizó la influencia de la relación de carga $(R C=k g$ de embutidos / kg de agua) en el descenso de la temperatura del agua. La Figura 3-7 muestra las predicciones numéricas del descenso de temperatura del agua vs. tiempo para distintas RC considerando un peso promedio de producto de $150 \mathrm{~g}$, un calor efectivo $\left(Q_{\mathrm{ef}}\right)$ de $1450 \mathrm{~W}$, temperatura inicial de agua $\left(T_{\text {ext,0}}\right)$ de $75^{\circ} \mathrm{C}$, un coeficiente de transferencia calórica $(h)$ de $828 \mathrm{~W} / \mathrm{m}^{2}$ ${ }^{\circ} \mathrm{C}$, y un volumen de agua de $30 \mathrm{~L}$. De acuerdo a lo esperado, el descenso de temperatura del agua se hizo apreciablemente más pronunciado cuando aumentaba la cantidad de embutidos a procesar. 

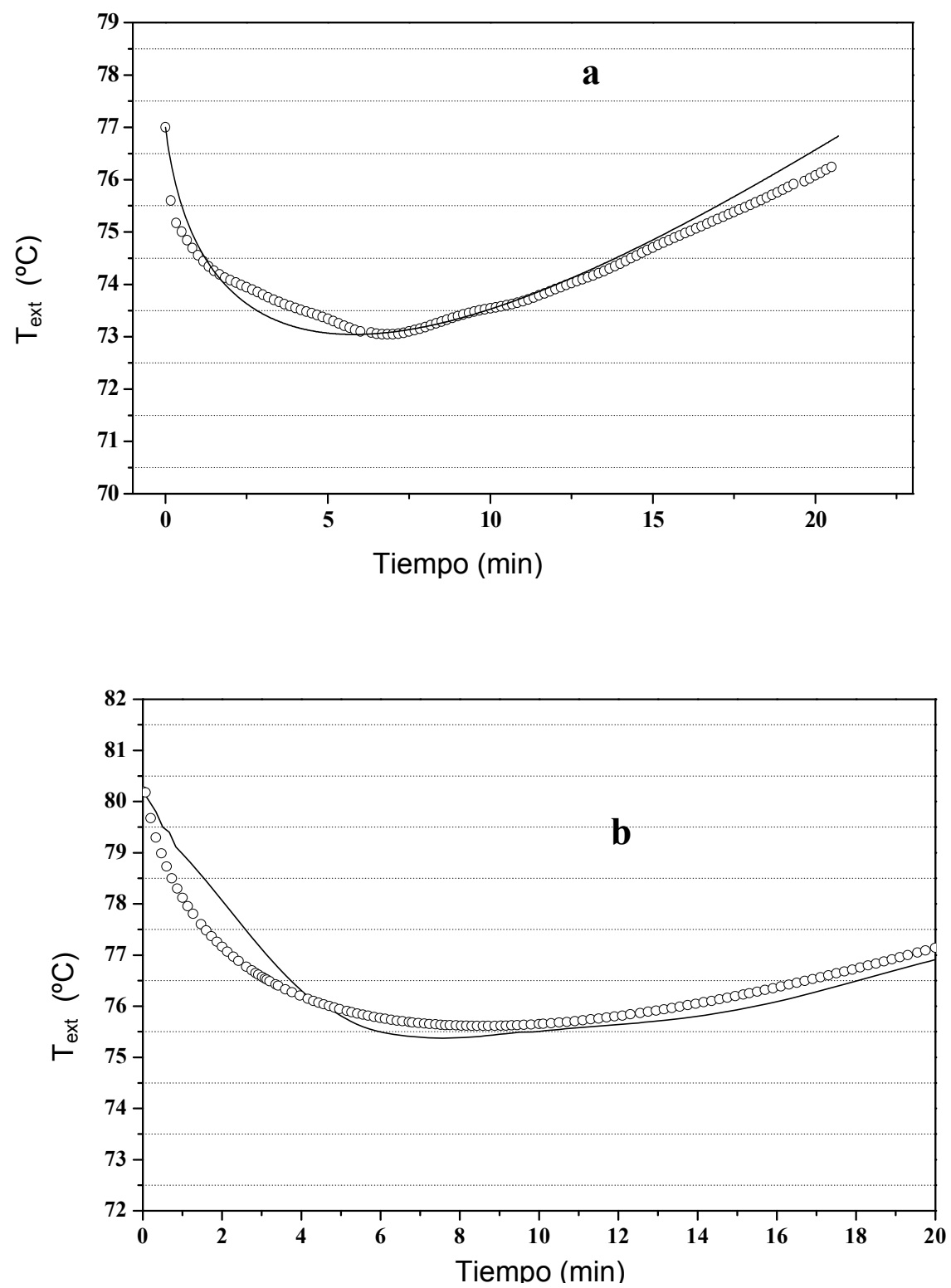

Fig. 3-6 Comparación entre las temperaturas predichas y experimentales del fluido calefaccionante que muestran el descenso de temperatura producido cuando se introduce la carga de producto. Descenso en la temperatura del fluido: a) Convección natural: : Temperatura de agua predicha, 0 : temperatura experimental, $\mathbf{h}=$ $828 \mathrm{~W} / \mathrm{m}^{2}{ }^{\circ} \mathrm{C}$, temperatura inicial $\left(T_{\text {ext }, 0}=77^{\circ} \mathrm{C}\right)$, masa de embutidos $3.5 \mathrm{~kg}$, y temperatura inicial de embutidos $\left(T_{i}=14^{\circ} \mathrm{C}\right)$. b) Convección Forzada, $h=1105 \mathrm{~W} / \mathrm{m}^{2}{ }^{\circ} \mathrm{C}$, temperatura inicial $\left(T_{\text {ext, },}=80.2^{\circ} \mathrm{C}\right)$, masa de embutidos $2 \mathrm{~kg}$, y temperatura inicial de embutidos $\left(T_{i}=\right.$ $13^{\circ} \mathrm{C}$ ). El volumen de agua para ambos casos fue $10 \mathrm{~L}$. 


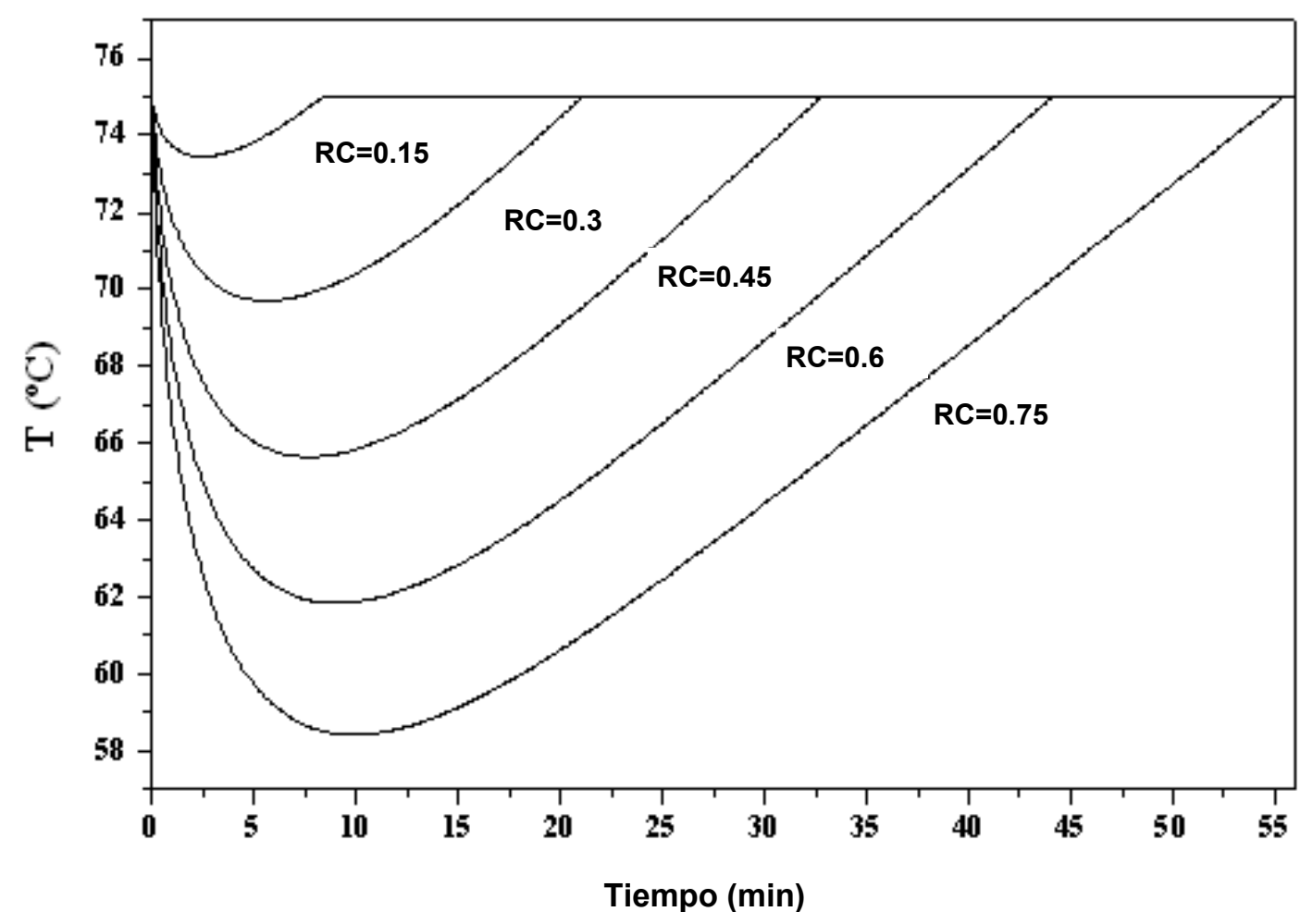

Fig. 3-7 Predicción numérica del descenso de temperatura del agua en función de la relación de carga ( $R C=\mathrm{kg}$ embutidos $/ \mathrm{kg}$ agua), considerando $\mathrm{h}=850 \mathrm{~W} / \mathrm{m}^{2}{ }^{\circ} \mathrm{C}, \mathrm{T}_{\text {ext, } 0}=$ $75^{\circ} \mathrm{C}$, temperatura inicial de producto; $T_{i}=13^{\circ} \mathrm{C}$.

\subsubsection{Efecto del descenso de la temperatura del agua en la inactivación térmica del microorganismo E. coli 0157:H7}

El descenso de la temperatura del agua al sumergirse una cierta cantidad de masa de embutidos (Fig. 3-7) lleva a que los tiempos del proceso para alcanzar un $\mathrm{VI}=12_{\log }$ sean más altos que los predichos para una condición de temperatura de fluido constante. En la Tabla 3-1 se pueden observar los tiempos de procesamiento térmico necesarios para alcanzar un valor de inactivación $(\mathrm{VI})=12_{\log }$ en función de la relación de carga $\mathrm{RC}$. En el caso donde la RC tiende a cero, la cantidad de agua es mucho mayor que la cantidad de producto y se podría asumir que la temperatura del fluido se mantiene constante a lo largo del todo el proceso. El tiempo mínimo requerido en este caso ideal para una temperatura de fluido de $75^{\circ} \mathrm{C}$, es de $20 \mathrm{~min}$. 
Como puede observarse en la Tabla 3-1 los tiempos mínimos de procesamiento que tiene en cuenta el descenso de la temperatura del fluido calefactor, son mucho mayores que 20 minutos por lo que adoptar el tiempo de proceso teórico un tiempo de 20 min se traduciría en VI menores a 12 log.

Tabla 3-1 Tiempos mínimos de proceso para alcanzar un $\mathrm{Vl}=12_{\log }$ para distintos valores de $R C$ considerando una temperatura inicial del fluido de $75^{\circ} \mathrm{C}, \mathrm{h}=850 \mathrm{~W} / \mathrm{m}^{2} \mathrm{C}$ y una temperatura inicial de producto de $13^{\circ} \mathrm{C}$.

\begin{tabular}{|cc|}
\hline RC (kg producto/kg agua) & Tiempo (min) \\
\hline 0.75 & 36.8 \\
0.60 & 32.2 \\
0.45 & 28.5 \\
0.30 & 25.9 \\
0.15 & 24.3 \\
0.00 & 20.0 \\
\hline
\end{tabular}

Resulta interesante remarcar que en el caso de utilizar los tiempos teóricos de proceso, donde no se considera un descenso de temperatura del fluido calefactor, el valor de inactivación alcanzado sería menor a $12_{\log }$. Por ejemplo asumiendo una temperatura de fluido constante, con una $\mathrm{RC}=0.45 \mathrm{y}$ $\mathrm{h}=1000 \mathrm{~W} / \mathrm{m}^{2}{ }^{\circ} \mathrm{C}$, los $\mathrm{VI}$ alcanzados serían de $2.7,1.8$, y 1.5 ciclos logarítmicos para una temperatura de fluido de 90,80 , y $70^{\circ} \mathrm{C}$, respectivamente, valores que están alejados de los $12 \log$ impuestos para este proceso. Esto demuestra la importancia de poder modelar adecuadamente el tratamiento térmico, ya que un insuficiente tiempo de proceso sería altamente riesgoso para el consumo de estos productos.

\subsubsection{Predicción de tiempos mínimos de proceso para distintas condiciones operativas.}

La combinación del balance microscópico con el balance macroscópico de energía, incorporando la cinética de inactivación microbiana, permitió 
calcular los nuevos tiempos mínimos de proceso para que el sistema alcance un $\mathrm{VI}=12_{\log }$, teniendo en cuenta el descenso de temperatura del agua.

Se observó que el efecto del coeficiente de transferencia de calor no era significativo en el rango evaluado (correspondiente a condiciones experimentales con y sin agitación mecánica), ya que las diferencias en los tiempos de calentamiento resultaron menores a 1 minuto. A modo de ejemplo se muestran en la Tabla 3.2 los tiempos mínimos de proceso (min) para dos valores de $\mathrm{h}$ y distintos valores de RC (kg producto/kg agua), considerando una temperatura inicial del fluido $\mathrm{T}=85^{\circ} \mathrm{C}$ :

Tabla 3-2 Efecto de RC y h en los tiempos de procesamiento térmico para alcanzar $\mathrm{Vl}=12_{\text {log. }}$.

\begin{tabular}{|c|c|c|}
\hline \multirow{2}{*}{$\begin{array}{c}\text { RC= (kg producto/kg } \\
\text { agua) }\end{array}$} & \multicolumn{2}{|c|}{$\mathbf{h = 8 0 0 ~ W / m ^ { 2 } { } ^ { \circ } \mathbf { C }}$} \\
\cline { 2 - 3 } & \multicolumn{2}{|c|}{ Tiempos de procesamiento (min) } \\
\hline 0.15 & 18.30 & $\mathbf{W} / \mathrm{m}^{2}{ }^{\circ} \mathbf{C}$ \\
0.30 & 20.30 & 18.16 \\
0.45 & 22.91 & 20.16 \\
0.60 & 26.16 & 22.68 \\
0.75 & 30.30 & 26.00 \\
& & 30.16 \\
\hline
\end{tabular}

Se aplicó un método de regresión "stepwise" (SYSTAT, USA) a los tiempos mínimos de proceso obtenidos mediante simulaciones numéricas, para encontrar una ecuación sencilla, que permita predecir los tiempos teniendo en cuenta la temperatura inicial del agua y la relación de carga $(R C)$. La ecuación que representa la superficie de respuesta del sistema y el coeficiente de correlación $(\mathrm{R})$ es la siguiente:

$$
\operatorname{tp}(\min )=63.276-0.526 \cdot T_{\text {ext, }}+23.206 \cdot R C^{2} \quad(R=0.968)
$$




\section{(F)}

donde $\mathrm{T}_{\text {ext, } 0}$ es la temperatura inicial del agua $\left({ }^{\circ} \mathrm{C}\right)$ y $\mathrm{RC}$ es la relación de carga (kg producto/kg agua). La superficie de respuesta se muestra en la Fig.3-8.

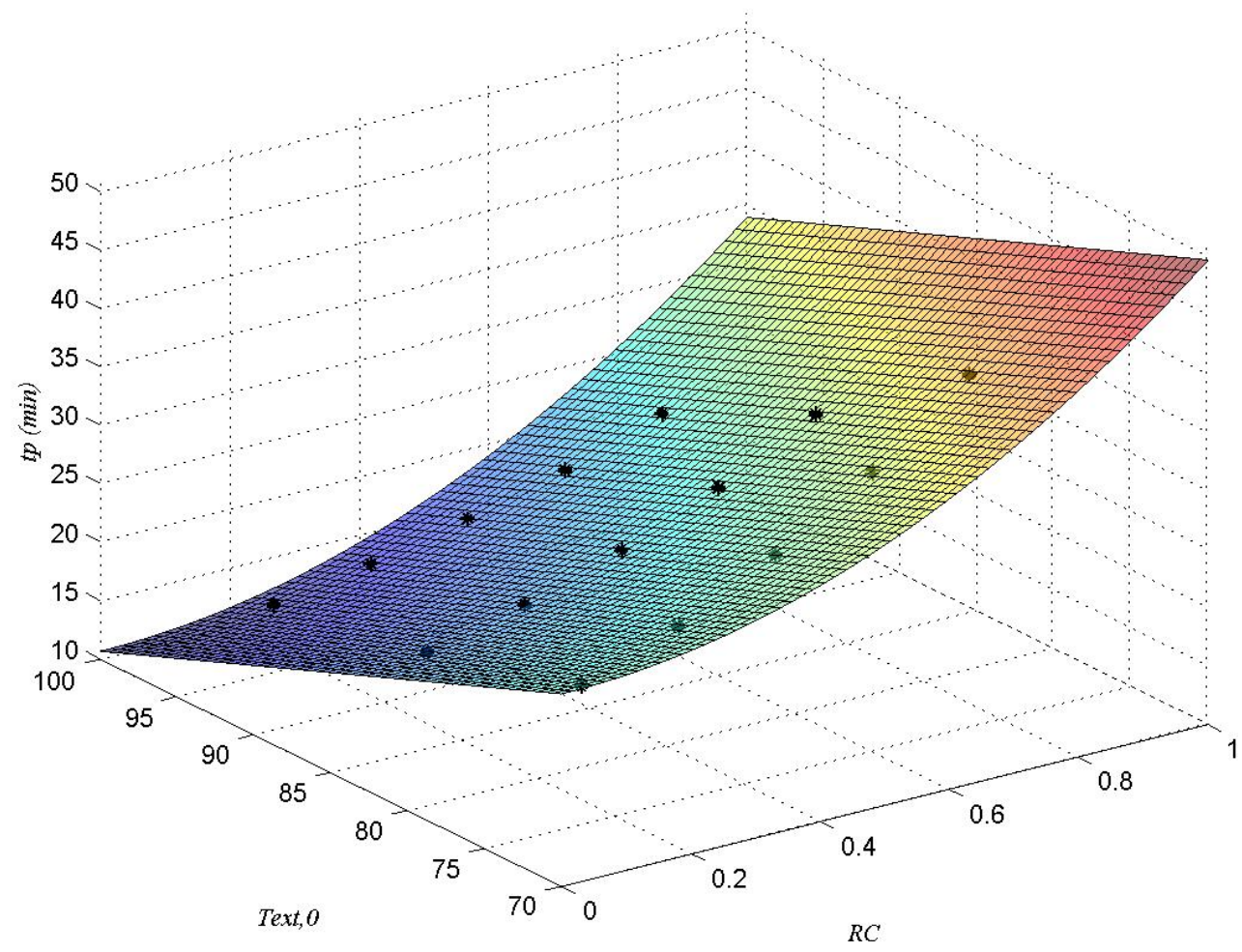

Fig. 3-8 Superficie de respuesta de los tiempos mínimos de proceso para alcanzar un VI de $12_{\log }$ en función de la temperatura inicial del agua $\left(T_{\text {ext, }, 0}\right)$ y la relación de carga $(R C=k g$ producto/kg agua) para una temperatura inicial de producto; $\mathrm{Ti}=13^{\circ} \mathrm{C}$, población microbiana inicial; $N_{0}=10^{6} \mathrm{UFC} /$ embutido, y un coeficiente de transferencia de calor; $h=$ $850 \mathrm{~W} / \mathrm{m}^{2}{ }^{\circ} \mathrm{C}$

La Ec. 3.19 es una herramienta útil que elimina la necesidad de ejecutar el programa computacional. El mismo permite observar que la influencia de la $\mathrm{RC}$ es mucho mayor que la de la temperatura inicial del fluido calefactor.

\subsection{Conclusiones}

Se implementó un programa computacional propio para establecer los requerimientos tiempo-temperatura en el calentamiento de embutidos, con el objetivo de lograr la inactivación térmica del microorganismo E. coli O157:H7 
$\left(\mathrm{VI}=12_{\mathrm{log}}\right)$ teniendo en cuenta condiciones de procesamiento industrial para la cual existe un descenso de la temperatura del fluido cuando se sumergen los productos. El modelo matemático resuelto mediante el método de elementos finitos se basó en resolver el balance microscópico diferencial de energía acoplando la cinética de destrucción microbiana y el balance macroscópico de energía.

En las simulaciones se consideró la relación de carga y el poder calorífico del sistema para la predicción del descenso de temperatura del agua en función del tiempo.

Se estudió el efecto de distintas relaciones de carga y coeficientes de transferencia de calor en el descenso de temperatura del agua y tiempos mínimos de proceso para alcanzar una reducción de $12_{\log }$ en la población microbiana.

A partir de las predicciones numéricas se logró obtener una ecuación simple que permite obtener los tiempos mínimos de proceso (tiempo necesario hasta alcanzar un $\mathrm{VI}=12_{\mathrm{log}}$ ), en función de la temperatura inicial del agua y la $\mathrm{RC}$.

El modelo numérico desarrollado constituye una herramienta útil para las plantas elaboradoras de este tipo de embutidos cuando se requiere determinar condiciones de tiempo-temperatura que aseguren la inocuidad del alimento. 
Capítulo 4 


\section{Resolución de problemas de transferencia de calor mediante el MEF en tres dimensiones}

\subsection{Introducción}

En los problemas de transferencia de calor en tres dimensiones la implementación de un código computacional resulta más compleja, en comparación con los problemas bidimensionales. Una de las razones más importantes se encuentra en la capacidad de integrar la información generada de la malla tridimensional; numeración de los elementos y nodos, con el código computacional propio y con las subrutinas de postprocesamiento. Por ende es fundamental entender y utilizar la información de malla incorporando subrutinas de preprocesamiento, para integrarla de manera eficiente y aprovecharla en los cálculos de postprocesamiento.

Los objetivos del presente capítulo de Tesis son:

> Desarrollar un código computacional en tres dimensiones utilizando el método de los elementos finitos para resolver el problema de transferencia de calor en estado transiente con condición de borde convectiva.

> Desarrollar una subrutina de preprocesamiento que compatibilice la información de malla con el programa principal.

$>$ Generar subrutinas de postprocesamiento que permitan calcular la variable dependiente (temperatura) o su gradiente (para el cálculo del flujo de calor) en cualquier punto del dominio y su valor integrado en la superficie o volumen del dominio estudiado.

- Validar el modelo en problemas tridimensionales comparando las predicciones del código computacional con soluciones analíticas y con resultados numéricos obtenidos a través de softwares comerciales.

El balance microscópico diferencial de energía en estado transiente en tres dimensiones con las condiciones iniciales y de contorno que se resolverán 
están dadas por (Carslaw y Jaeger, 1959):

$$
\begin{aligned}
& \rho \operatorname{Cp} \frac{\partial \mathrm{T}}{\partial \mathrm{t}}=\mathrm{k}\left(\frac{\partial^{2} \mathrm{~T}}{\partial \mathrm{x}^{2}}+\frac{\partial^{2} \mathrm{~T}}{\partial \mathbf{y}^{2}}+\frac{\partial^{2} \mathbf{T}}{\partial \mathbf{z}^{2}}\right) \quad \text { en } \Omega \\
& \begin{array}{lll}
\mathrm{T}=\mathrm{T}_{0} & \mathrm{t}=0 \quad \text { en } \Omega
\end{array} \\
& -k\left(\frac{\partial T}{\partial x} \cdot n_{x}+\frac{\partial T}{\partial y} \cdot n_{y}+\frac{\partial T}{\partial z} \cdot n_{z}\right)=h\left(T-T_{\text {ext }}\right) \quad t \geq 0 \quad \text { en } \delta \Omega_{1}
\end{aligned}
$$

Aplicando la formulación variacional y representando a la temperatura mediante un espacio de dimensión finito $\mathrm{V}_{\mathrm{h}}$ con funciones de forma $\mathrm{H}$, donde $\mathrm{T}_{\mathrm{h}} \mathrm{H}_{\mathrm{H}} \mathrm{H} \cdot \mathrm{T}$ (Método de Galerkin) (Zienkiewicz y Taylor, 1994a; Zienkiewicz y Taylor, 1994b; Bathe, 1996) se obtiene:

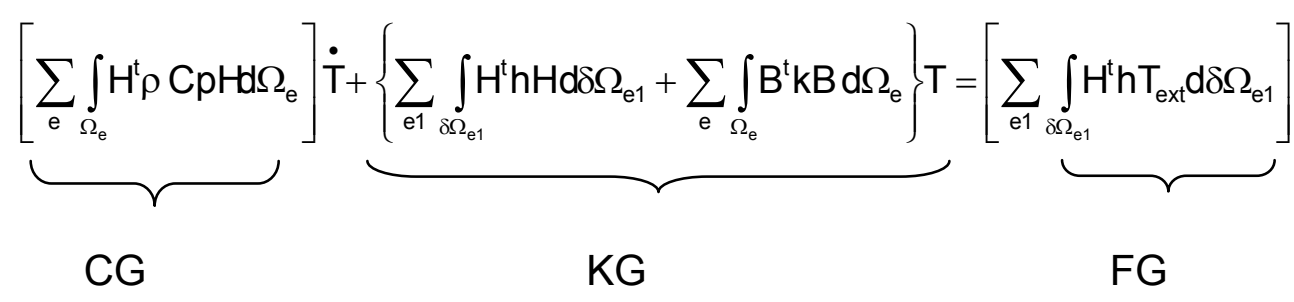

donde $C G, K G$, y $F G$ son la matriz global de capacitancia, conductancia y vector de fuerzas globales, respectivamente. En forma más compacta se puede escribir la Ec. 4.4 como:

$\mathrm{CG} \dot{\mathrm{T}}+\mathrm{KGT}=\mathrm{FG}$

El sistema semi-discreto (Ec. 4.5) es un sistema rígido de ecuaciones diferenciales ordinarias, cuya discretización temporal se describió en el Capítulo 2 utilizando el método $\alpha$, adoptando un método implícito con $\alpha=1$ (Johnson, 1986) para su resolución. 


\subsection{Generación de la malla y preprocesamiento}

La discretización espacial del dominio se realizó mediante un generador de malla utilizando elementos tetraédricos lineales. La información de la malla se transfiere generalmente utilizando tres matrices; la matriz de nodos $\mathbf{p}$, matriz de tetraedros, tm, y la matriz de borde e. La matriz de nodos representa las coordenadas $\mathrm{x}, \mathrm{y}, \mathrm{z}$ de los puntos o nodos que forman la grilla y está dada en tres columnas, donde el número de la fila corresponde con el número del nodo. La matriz tm de tetraedros establece la conectividad del elemento con los nodos, es una matriz de $4 \times \mathrm{N}$, siendo $\mathrm{N}$ el número de elementos, donde los nodos tienen un orden específico en cada fila, según el orden establecido en el tetraedro de referencia, descripto en la Fig. 4-1.

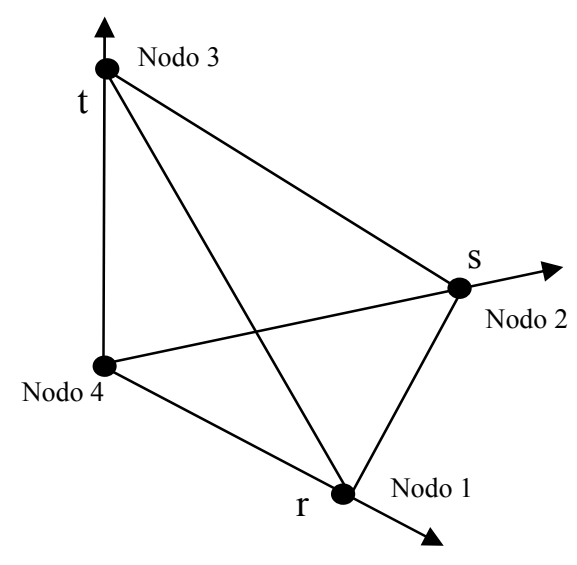

Fig. 4-1 Tetraedro de referencia

Las funciones de forma lineales son:

$\mathrm{h} 1=\mathrm{r} \quad 0 \leq \mathrm{r} \leq 1$

$\mathrm{h} 2=\mathrm{s}$

$0 \leq \mathrm{s} \leq 1$ 
La matriz de borde e contiene información de los nodos (tres) que son los vértices de la superficie triangular en contacto con la interfase $\delta \Omega$. Sin embargo no existe información correspondiente a qué elemento de la malla pertenece. Esto es un tema importante para calcular las matrices elementales si se tiene un programa computacional que calcula el lazo de las matrices elementales y el ensamble por elemento. También es importante saber a qué elemento pertenece, si se realiza un postprocesamiento, donde el objetivo es calcular el campo gradiente de temperaturas en la superficie, ya que se requiere además de la temperatura de los tres nodos de la superficie expuesta, la temperatura del nodo interno que forma el tetraedro.

La superficie triangular en contacto con la interfase presenta tres de los cuatro vértices. Estos tres nodos se encuentran en la matriz $\mathbf{t m}$ en un orden particular. Al ser 4 los nodos que componen un tetraedro y 3 los vértices de la superficie expuesta, el programa de preprocesamiento debe incluir tantos lazos "if" como el número de combinaciones en las que se pueden encontrar juntos los tres nodos en la matriz tm, lo cual suma 24 (seis por cada una de las posibles superficies triangulares).

El programa de preprocesamiento ayuda a integrar la información de la superficie requerida por el programa principal. A modo de ejemplo de uno de los 24 lazos "if" en lenguaje Matlab, considerando que length(e) es el número de elementos de borde y length (tm) es igual al número total de elementos:

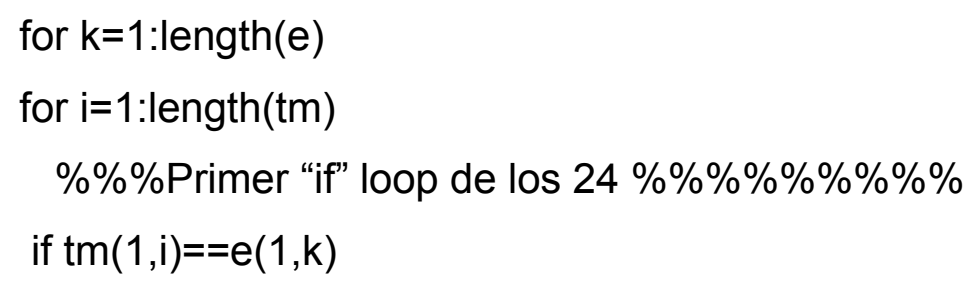




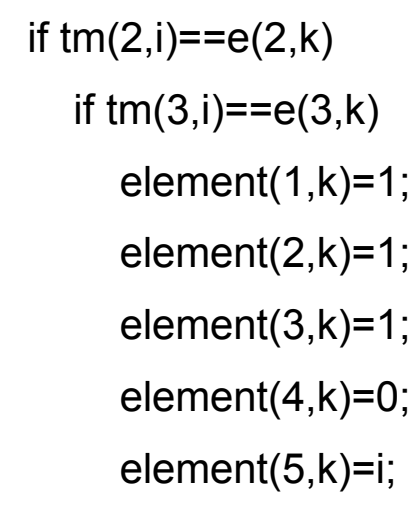

end

end

end

$\% \% \% \%$ fin del primer "if" loop

El número de elemento se acumula en la matriz element en la quinta fila. Se asigna el valor cero al nodo que no pertenece a la superficie triangular expuesta; en este caso la función de forma h4 es cero, por ende la fila 4 de la matriz element es cero. A la condición h4 $=0$ se la denomina triángulo de superficie $S_{4}$, que involucra los nodos locales 1,2 , y 3 . La superficie $S_{1}$ involucra los nodos locales 2, 3 y 4 (h1 = 0), $S_{2}$ involucra los nodos locales 1, 4, y 3 (h2 = 0), y $S_{3}$ los nodos locales 1, 2, 4 (h3 = 0). La Fig. 4-2 ilustra todos los casos posibles de superficies triangulares y los nodos que intervienen en cada caso. La Fig. 4-2 d) representa el ejemplo dado con anterioridad y corresponde a $\mathrm{S}_{4}$.

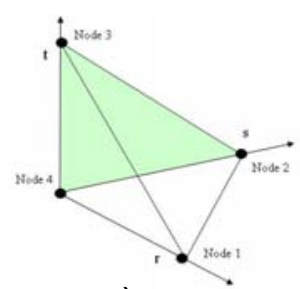

a)

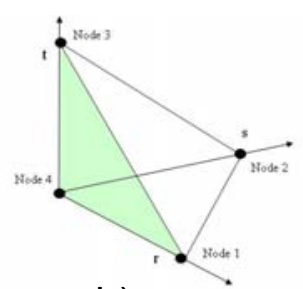

b)

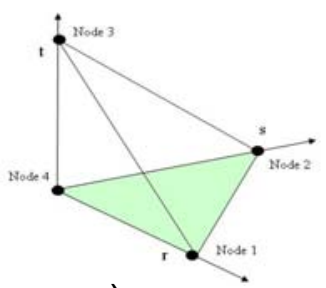

c)

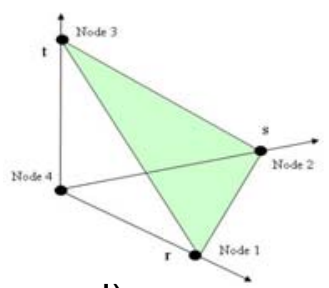

d)

Fig. 4-2: Superficies triangulares y nodos locales que constituyen cada tipo de superficie. a) $S_{1}$, b) $S_{2}$, c) $S_{3}$, d) $S_{4}$ 


\subsection{Programa en elementos finitos}

La información de malla preprocesada se ha utilizado en el programa computacional principal cuya estructura se muestra en la Fig. 4-3. El esquema de diagrama de flujo describe cómo está desarrollado el código computacional, donde $\operatorname{det}(\mathbf{J})$ es el determinante del la matriz Jacobiana, $|\vec{n}|$ es el módulo del vector unitario normal exterior, y $\mathbf{w}$ es el vector que contiene los pesos de la regla de cuadratura utilizada para la integración numérica.

Cuando se completa el cálculo de las matrices elementales se prosigue con el aporte de las integrales de borde, siempre y cuando el tetraedro presente alguna superficie expuesta a la condición de borde. La matriz element tiene la información correspondiente a qué tipo de superficie está expuesta: $\mathrm{S}_{1}$, $S_{2}, S_{3} \circ S_{4}$. En un espacio tridimensional la integración de una función sobre una superficie se representa de la siguiente forma (Leithold, 1998):

$$
\iint_{S} f d S=\iint_{A} f|n| d A
$$

En el caso que el elemento tenga un lado $S_{4}$ (Ver Fig. 4-4), el vector normal a la superficie se obtiene a través del producto vectorial:

$\vec{n}=v 1 \times v 2$ 


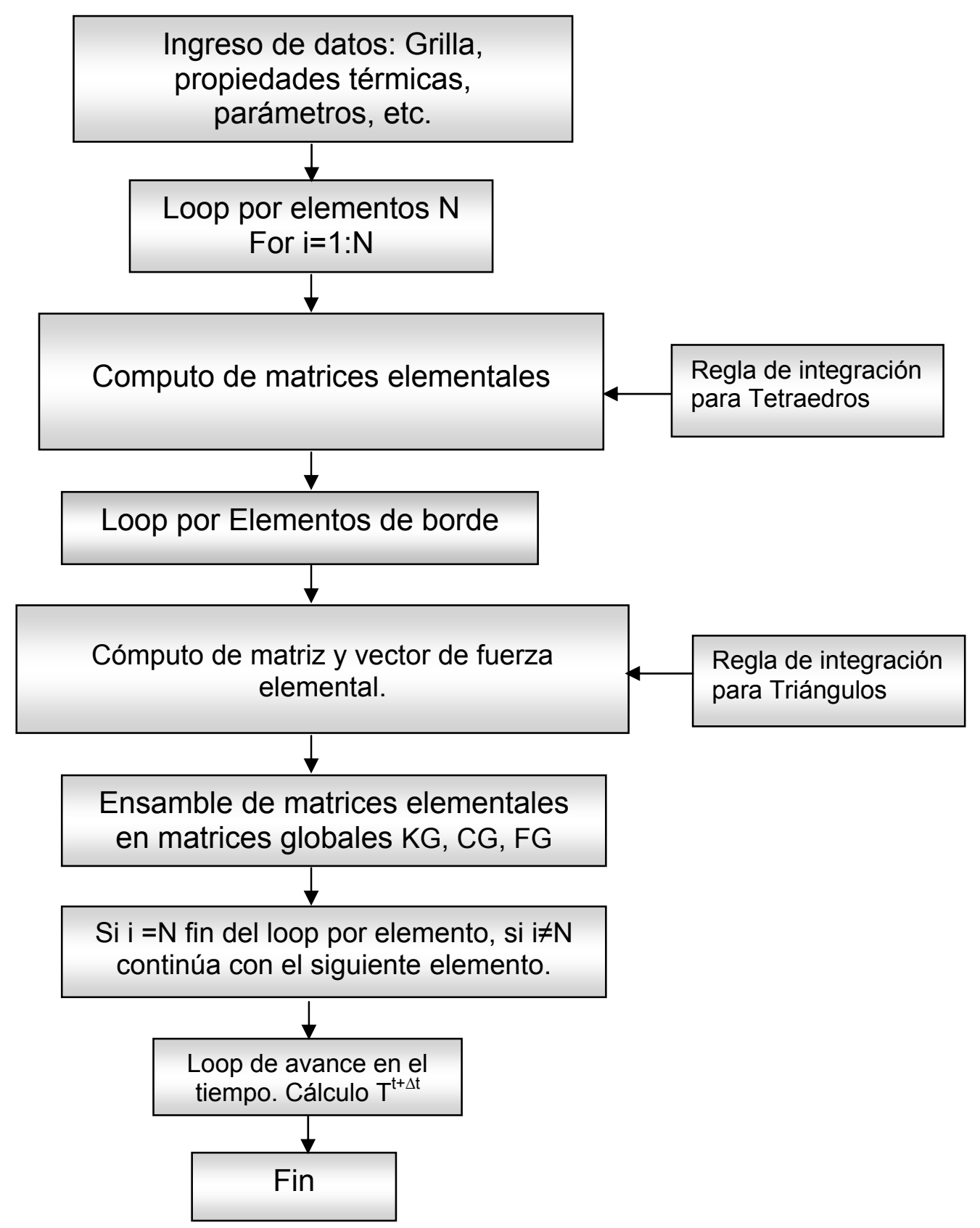

Fig. 4-3. Esquema del diagrama de flujo del programa computacional utilizando el MEF 


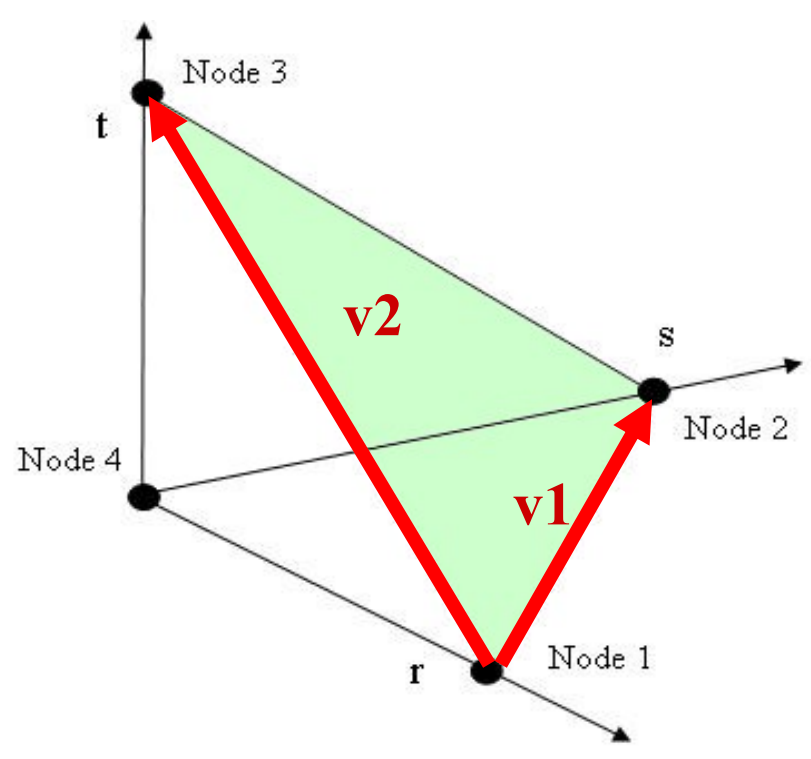

Fig. 4-4 Elemento con superficie expuesta a condición de borde convectiva de tipo $\mathrm{S}_{4}$, donde intervienen los nodos 1, 2, y 3.

El plano que representa $\mathrm{S}_{4}$ es:

$c 1 \cdot(x-x 1)+c 2 \cdot(y-y 1)+c 3 \cdot(z-z 1)=0$

donde

$c 1=(y 3-y 1) \cdot(z 2-z 1)-(z 3-z 1) \cdot(y 2-y 1)$

$c 2=(z 3-z 1) \cdot(x 2-x 1)-(x 3-x 1) \cdot(z 2-z 1)$

$c 3=(x 3-x 1) \cdot(y 2-y 1)-(y 3-y 1) \cdot(x 2-x 1)$

Existe la posibilidad de encontrar una superficie triangular que sea perpendicular a algún plano coordenado, imposibilitando su proyección sobre ese plano (en ese caso ci es próximo a cero). Por esta razón mediante la siguiente sentencia en Matlab:

[Valor, Imax $]=\max (\operatorname{abs}(\mathrm{c}))$ donde $\mathrm{c}=[\mathrm{c} 1 \mathrm{c} 2 \mathrm{c} 3]$

se identifica el valor ci con i=1, 2 o 3 que presenta el valor máximo. 
Si se identifica el valor c1 como el valor máximo, el vector normal resulta:

$\vec{n}=\left(1, \frac{-c 2}{c 1}, \frac{-c 3}{c 1}\right)$

Si se elige $c 1 \neq 0$ se evita la división por cero para el cálculo del vector normal, es decir, se evita proyectar sobre el plano perpendicular a la superficie.

En ese caso se proyecta la superficie sobre el plano "yz". El Jacobiano de la transformación Te: $y=y(r, s), z=z(r, s)$ se define como:

$$
J=\left(\begin{array}{ll}
\frac{\partial y}{\partial s} & \frac{\partial y}{\partial r} \\
\frac{\partial z}{\partial s} & \frac{\partial z}{\partial r}
\end{array}\right)
$$

Recordar que $\mathrm{r}$, s son las variables utilizadas en el triángulo de referencia.

Análogamente si c2 o c3 es el valor máximo se proyecta sobre los planos zx, xy, respectivamente, definiendo el Jacobiano correspondiente a cada caso.

Finalmente, se evalúa mediante integración numérica utilizando la regla de cuadratura para triángulos mediante la siguiente expresión (Bathe, 1996):

$$
\iint f d S \cong \sum_{i} f(r(i), s(i)) w(i) \operatorname{det}(J) \cdot 0.5 \cdot|\vec{n}|
$$

donde el índice i indica el punto de integración utilizado para triángulos, w(i) es el peso de los puntos de cuadratura elegidos (Hughes, 1987). La función f(r(i), $\mathrm{s}(\mathrm{i}))$, representa un polinomio compuesta por la multiplicación de las funciones de forma.

El esquema utilizado para la discretización en el tiempo fue mediante el 
Método $\alpha$ (Segerlind, 1984), adoptando $\alpha=1$, por lo que se implementó un método implícito. El paso en el tiempo fue de $1 \mathrm{~s}(\Delta \mathrm{t}=1 \mathrm{~s})$.

\subsection{Postprocesamiento}

Los resultados pueden obtenerse creando una subrutina de postprocesamiento que por ejemplo permita calcular la temperatura en cualquier punto del dominio. Se ha utilizado una sentencia (en lenguaje de Matlab) en la cual la información alimentada a la subrutina son las coordenadas $\mathrm{x}, \mathrm{y}, \mathrm{z}$ del punto de interés (al cual llamaremos punto $\mathbf{M}$ ) y las matrices $\mathbf{p}$ y tm de la grilla. La información de salida son los nodos que forman el elemento que contiene el punto $\mathbf{M}$ y las funciones de interpolación evaluadas en dicho punto. La sentencia utilizada es:

function [h1,h2,h3,h4, nodo1, nodo2,nodo3,nodo4]=pospro(x, y, z, p, tm)

Dentro de la función "pospro" se calcula la distancia del punto $\mathbf{M}$ al centro geométrico de cada tetraedro, encontrando cuál es el elemento que minimiza la distancia al cuadrado. El cálculo de la distancia se realiza de manera sencilla simplemente restando las coordenadas del punto $\mathbf{M}$ al centro geométrico de cada elemento.

Una vez que se identifica el elemento se sabe cuáles son los nodos que participan y sus coordenadas, por lo que la coordenada $\mathrm{x}$ del punto $\mathbf{M}$ se puede escribir como:

$\mathrm{x}=\mathrm{xnodo} 1 \cdot \mathrm{h} 1+\mathrm{xnodo} 2 \cdot \mathrm{h} 2+\mathrm{xnodo} 3 \cdot \mathrm{h} 3+\mathrm{xnodo} 4 \cdot \mathrm{h} 4$

Análogamente se pueden escribir las coordenadas $\mathrm{y}, \mathrm{z}$ del punto $\mathbf{M} \mathbf{y}$ combinando las funciones de interpolación se obtiene: h4=1-h1-h2-h3, resultando en un sistema simple de tres ecuaciones con tres incógnitas 
(h1,h2,h3). Una vez resuelto el sistema, la temperatura en el punto $\mathbf{M}$ se expresa de la siguiente manera:

$\mathrm{T}=\mathrm{T}($ nodo1 $) \cdot \mathrm{h} 1+\mathrm{T}($ nodo 2$) \cdot \mathrm{h} 2+\mathrm{T}($ nodo3 $) \cdot \mathrm{h} 3+\mathrm{T}($ nodo 4$) \cdot \mathrm{h} 4$

Si se quiere calcular la temperatura media del dominio se necesita almacenar el vector de temperatura de los nodos y calcular el volumen, ya que la temperatura media se define como:

$$
\bar{T}=\frac{\int_{V} T d V}{\int_{V} d V}
$$

donde $\mathrm{V}$ es el volumen del dominio.

Se realiza la integración de la temperatura y el volumen de cada elemento tetraédrico utilizando los puntos de integración para un tetraedro (ver las siguientes sentencias codificadas en lenguaje Matlab) y las temperaturas nodales $(\mathrm{T} 1, \mathrm{~T} 2, \mathrm{~T} 3, \mathrm{~T} 4)$.

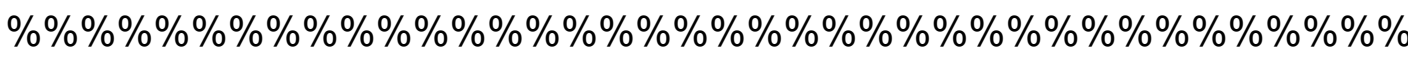

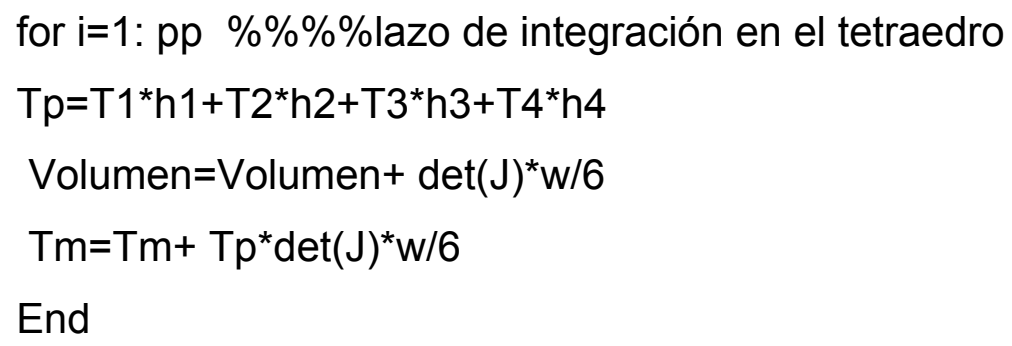

Una vez que finaliza el loop por elemento la temperatura media se calcula como: 


$$
\overline{\mathrm{T}} \cong \frac{\mathrm{Tm}}{\text { Volumen }}
$$

Para el cálculo del flujo de calor (energía/(área tiempo)) sobre la superficie es necesario calcular el gradiente de temperatura. Como las funciones de interpolación son funciones lineales, el gradiente será un vector cuyas componentes serán constantes. Se requiere conocer el valor de temperatura en los nodos del tetraedro, el cual contiene una de sus superficies en contacto con la condición de borde y el vector normal unitario a dicha superficie. A continuación se muestran algunas de las sentencias codificadas en Matlab.

$\% \%$ cálculo del gradiente y flujo de calor

$\mathrm{Tq}=[\mathrm{T}(\operatorname{tm}(1, n), 1), \mathrm{T}(\operatorname{tm}(2, \mathrm{n}), 1), \mathrm{T}(\operatorname{tm}(3, n), 1), \mathrm{T}(\operatorname{tm}(4, n), 1)]$;

$$
\operatorname{gradT}=\operatorname{inv}(J)^{*} \mathrm{Hr}^{*} \mathrm{Tq}^{\prime}
$$

$\mathrm{q}=-\mathrm{k}^{*}(\operatorname{gradT})^{*}$ normal

$\% \% \% \% \% \% \% \% \% \%$

La matriz $\mathrm{tm}$ corresponde a la descripta en la Sección 4.2, donde el índice $\mathrm{n}$ corresponde al elemento que presenta una superficie en contacto con el borde. La matriz $\mathrm{Hr}$ contiene las derivadas con respecto a $r$ y $s$ de las funciones de interpolación (ver Capítulo 2) e inv $(\mathrm{J})$ representa la inversa de la matriz Jacobiana.

\subsection{Resultados y discusión}

\subsubsection{Validación del código computacional mediante solución analítica para esfera.}

Para corroborar la convergencia y exactitud de las predicciones numéricas utilizando diferentes mallas, se compararon los resultados 


\section{(F)}

numéricos con la solución analítica. Estas mallas presentaban distintos grados de refinamiento en su discretización espacial con el objetivo de verificar que las diferencias entre la predicción numérica y la solución analítica disminuían a medida que aumentaba el número de elementos que componían la malla. Esto implica la convergencia de la solución numérica hacia la solución analítica. El código computacional desarrollado en lenguaje Matlab 6.5 se presenta en el Apéndice 4-1 al final del presente Capítulo.

Se utilizó la solución analítica de la ecuación de transferencia de energía con condición de borde convectiva para una esfera (Welty, 1974, Carslaw y Jaeger, 1959).

$$
\frac{T-T_{\text {ext }}}{T_{0}-T_{e x t}}=4 \cdot \frac{R}{r} \cdot \sum_{n=1}^{\infty} e^{-\delta_{n}^{2} \cdot\left(\frac{\alpha t}{R^{2}}\right)} \cdot \sin \left(\delta_{n} \cdot \frac{r}{R}\right) \cdot \frac{\sin \left(\delta_{n}\right)-\delta_{n} \cdot \cos \left(\delta_{n}\right)}{2 \delta_{n}-\sin \left(2 \delta_{n}\right)}
$$

donde $\delta_{\mathrm{n}}$ son las raíces de la ecuación característica:

$$
1-\delta_{n} \cdot \cot \left(\delta_{n}\right)=\frac{h R}{k}
$$

Se alimentaron las propiedades termofísicas de un material acrílico $\left(\mathrm{k}=0.2075 \mathrm{~W} / \mathrm{m}^{\circ} \mathrm{C}, \quad \mathrm{Cp}=1464 \mathrm{~J} / \mathrm{kg}^{\circ} \mathrm{C}, \quad \rho=1180 \mathrm{~kg} / \mathrm{m}^{3}\right)$. Se consideró la temperatura inicial del sólido $=20^{\circ} \mathrm{C}$, la temperatura del fluido $=60.3^{\circ} \mathrm{C}$, el coeficiente de transferencia calórica $\mathrm{h}=55 \mathrm{~W} / \mathrm{m}^{2}{ }^{\circ} \mathrm{C}$, para un radio de la esfera $\mathrm{R}=0.0152 \mathrm{~m}$.

La malla utilizada fue obtenida a través de un generador de grilla llamado "DistMesh" (Perssons y Strang, 2004). Este generador es un programa codificado en MATLAB que permite obtener elementos tetraédricos de excelente calidad comparado con los obtenidos mediante la triangulación de Delayuny, que se utiliza por ejemplo en el COMSOL AB, 2005. La Fig. 4-5 muestra dos de las mallas utilizadas para los cálculos. 


\section{(F)}

C I D C A

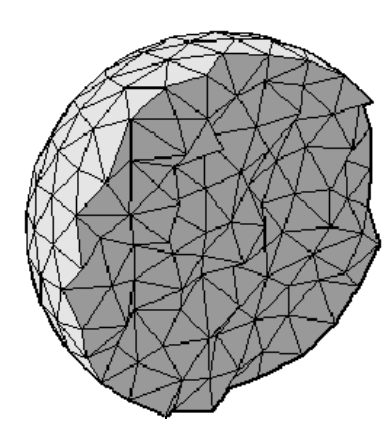

a)

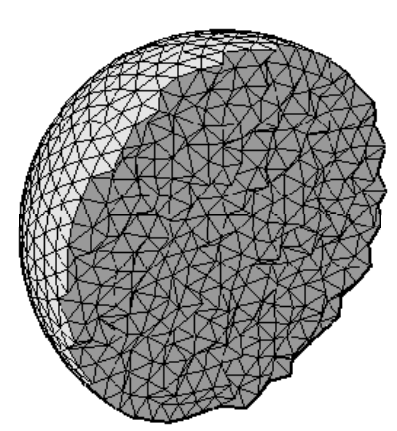

b)

Fig. 4-5 Mallas utilizadas en el programa computacional a) Malla 2 b) Malla 4.

Se calculó el error relativo porcentual $\left(e \%=\left(\frac{T_{a}-T_{n}}{T_{a}}\right) \cdot 100\right)$ para cada tiempo en el punto central $(r=0)$, medio $(r=R / 2)$, y el borde $(r=R)$, donde $T_{a}$ es la temperatura obtenida en forma analítica y $T_{n}$ es la temperatura predicha por el modelo numérico utilizando el MEF. Se calculó la norma infinito, \|\|$_{\infty}$, que corresponde al valor absoluto máximo del vector error relativo porcentual $(\mathrm{e} \%)$ para todos los tiempos comparados.

Se estimó el tiempo requerido de máquina para resolver el problema numérico (tiempo ${ }_{\mathrm{cpu}}$ ) dado en min. (ver Tabla 4-1) utilizando distintas mallas. La PC utilizada para la simulación fue una Intel(R) Core(TM) 26300 con una velocidad de procesador de $1.86 \mathrm{GHz}$ y una memoria RAM de $2 \mathrm{~GB}$. A modo de ejemplo se visualiza en la Fig. 4-6 la temperatura adimensional obtenida en forma analítica y numérica al utilizarse la Malla 2.

Se puede observar que la $\|\mathrm{e}\|_{\infty}$ disminuye y por lo tanto existe una mejora en la aproximación numérica al aumentar el número de nodos de la malla, sin embargo los tiempos de ejecución aumentan en forma considerable. La malla que podría considerarse óptima es aquella que permite mantener el esfuerzo computacional en un valor aceptable con un error numérico mínimo. 


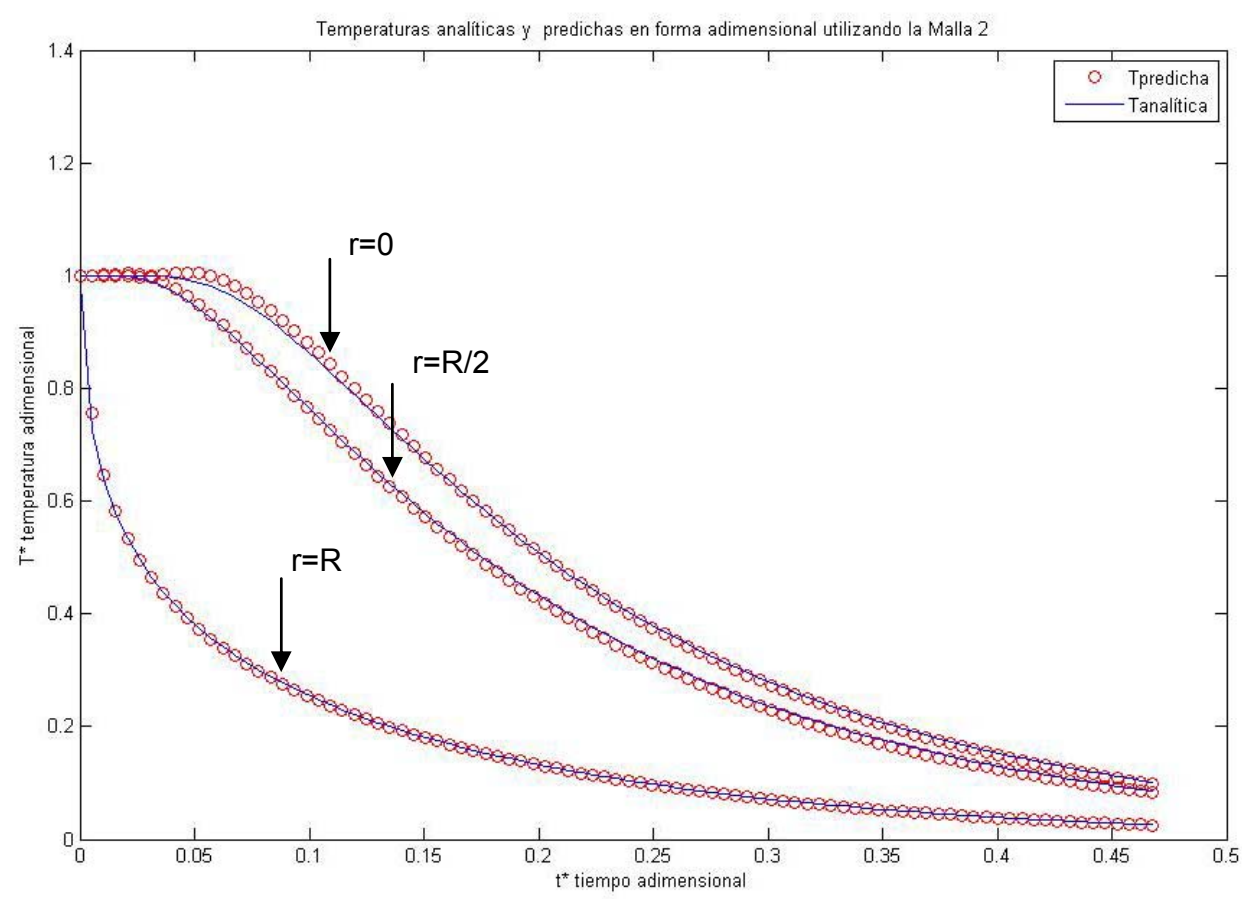

Fig. 4-6. Temperaturas adimensionales $\left(T^{*}=\left(T(r, t)-T_{\text {ext }}\right) /\left(T_{0}-T_{\text {ext }}\right)\right)$ calculadas a través de la solución analítica y el modelo numérico en elementos finitos en función del tiempo adimensional $t^{*}=\alpha t / R^{2}$ para distintas posiciones $(r=0, r=R / 2, y=R)$ de la esfera utilizando la malla 2. $\left(\mathrm{h}=55 \mathrm{~W} / \mathrm{m}^{2}{ }^{\circ} \mathrm{C}\right)$

Tabla 4-1: Máximos valores absolutos (norma infinito del error relativo porcentual) $\left(e \%=\left(\frac{T_{a}-T_{n}}{T_{a}}\right) \cdot 100\right)$ para distintas mallas generadas para una esfera y tiempos de ejecución requeridos tiempo $\mathrm{cpu}_{\mathrm{cu}}(\mathrm{min})$.

\begin{tabular}{|c|c|c|c|c|}
\hline$\|\mathrm{e}\|_{\infty}(\%)$ & $r=0$ & $r=R / 2$ & $r=R$ & $\begin{array}{l}\text { tiempo }_{\text {cpu }} \\
\text { (min) }\end{array}$ \\
\hline $\begin{array}{l}\text { Malla } 1 \\
142 \text { nodos, } 515 \text { elementos }\end{array}$ & 10.92 & 8.75 & 6.89 & 0.23 \\
\hline $\begin{array}{l}\text { Malla } 2 \\
592 \text { nodos, } 2726 \text { elementos }\end{array}$ & 3.75 & 1.70 & 3.86 & 1.02 \\
\hline $\begin{array}{l}\text { Malla } 3 \\
1908 \text { nodos, } 9573 \text { elementos }\end{array}$ & 0.95 & 0.73 & 0.84 & 11.92 \\
\hline $\begin{array}{l}\text { Malla } 4 \\
4500 \text { nodos, } 23695 \text { elementos }\end{array}$ & 0.37 & 0.32 & 0.23 & 66.66 \\
\hline
\end{tabular}


En este caso se eligió la Malla 3, ya que el error numérico fue menor al $1 \%$ y el tiempo ${ }_{\text {cpu }}$ aceptable.

\subsubsection{Validación del código computacional mediante solución analítica para cilindro finito.}

Se compararon las predicciones numéricas con las soluciones analíticas para un cilindro finito utilizando tres tipos de mallas.

La solución analítica de la ecuación de calor con condición de borde convectiva para un cilindro infinito de acrílico es (Welty, 1974; Carslaw y Jaeger, 1959).

$$
\frac{T-T_{\text {ext }}}{T_{0}-T_{\text {ext }}}=2 \cdot \sum_{n=1}^{\infty} \frac{e^{-\delta_{n}{ }^{2} \cdot\left(\frac{\alpha t}{R^{2}}\right)}}{\delta_{n}} \cdot \frac{J_{1}\left(\delta_{n}\right) J_{0}\left(\delta_{n} \cdot \frac{r}{R}\right)}{J_{0}{ }^{2}\left(\delta_{n}\right)+J_{1}{ }^{2}\left(\delta_{n}\right)}
$$

donde $\delta_{\mathrm{n}}$ satisface:

$\delta_{\mathrm{n}} \frac{\mathrm{J}_{1}\left(\delta_{\mathrm{n}}\right)}{\mathrm{J}_{0}\left(\delta_{\mathrm{n}}\right)}=\frac{\mathrm{hR}}{\mathrm{k}}$

y $J_{1}$ y $J_{0}$ son las funciones de Bessel de orden uno y cero respectivamente evaluadas en $\delta_{n}$.

Para una placa plana infinitamente larga y ancha la solución analítica de la ecuación de calor con condición de borde convectiva es:

$$
\frac{T-T_{\text {ext }}}{T_{0}-T_{\text {ext }}}=2 \sum_{n=1}^{\infty} e^{-\delta_{n}^{2} \cdot\left(\frac{\alpha t}{L^{2}}\right)} \cdot \frac{\sin \left(\delta_{n}\right) \cdot \cos \left(\delta_{n} \cdot \frac{x}{L}\right)}{\delta_{n}+\sin \left(\delta_{n}\right) \cdot \cos \left(\delta_{n}\right)}
$$

donde $L$ es el semi espesor de la placa, y $\delta_{n}$ satisface la siguiente ecuación característica: 


$$
\delta_{\mathrm{n}} \cdot \tan \left(\delta_{\mathrm{n}}\right)=\frac{\mathrm{hL}}{\mathrm{k}}=\mathrm{Bi}
$$

Aplicando la regla de Newman se obtiene la solución para un cilindro finito multiplicando la solución analítica para un cilindro infinito y la solución analítica para una placa plana infinitamente larga y ancha.

La altura total del cilindro fue de $0.0304 \mathrm{~m}$ y el radio $0.0152 \mathrm{~m}$. Se utilizaron las mismas propiedades térmicas y condiciones iniciales que en el caso de la esfera (4.5.1). La Fig. 4-7 muestra un ejemplo dos de las mallas utilizadas. La norma infinito del error porcentual fue calculado en tres puntos del dominio;

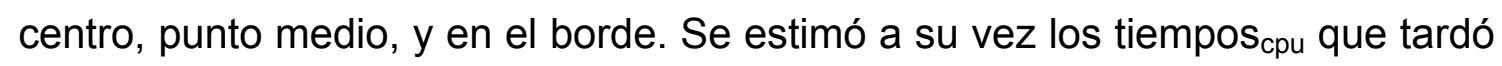
el código computacional en correr para cada malla (ver Tabla 4-2).

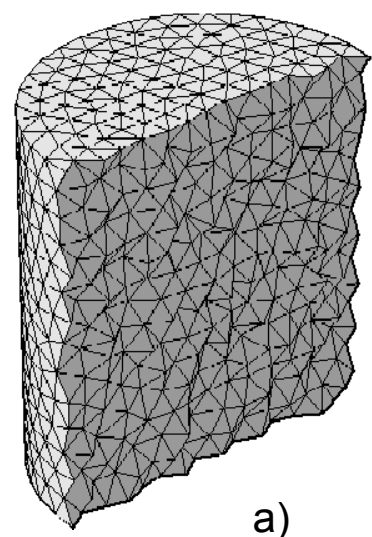

a)

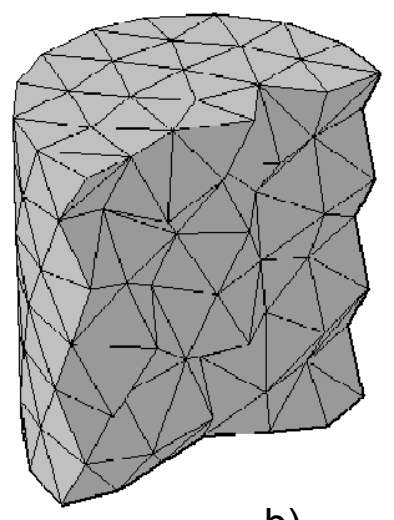

b)

Fig. 4-7: Mallas de cilindros finitos utilizadas en el programa computacional, a) Malla 3 b) Malla 1

En la Fig. 4-8 se puede observar la buena concordancia que existe en tres puntos del dominio (centro, punto interior, y borde), entre la temperatura adimensional obtenida en forma analítica y la simulada numéricamente en función del tiempo (adimensional), para el caso en que se utilizó la Malla 2. 


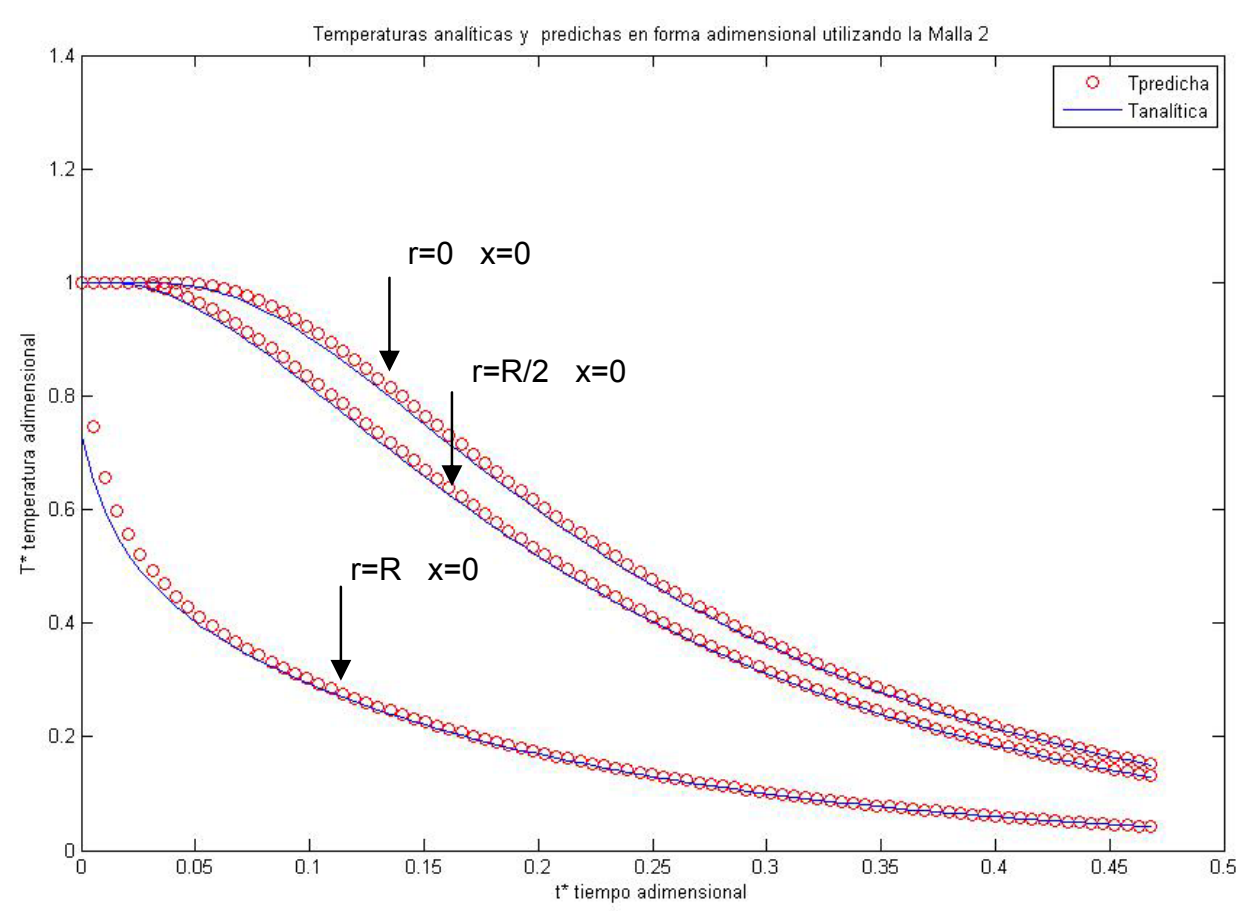

Fig. 4-8. Temperaturas adimensionales calculadas a través de la solución analítica y el modelo numérico en elementos finitos en función del tiempo adimensional $t^{*}=\alpha t / L^{2}$ para distintas posiciones (centro, $(r=0, x=0)$, punto medio $(r=R / 2, x=0)$ y borde $(r=R, x=0)$ ) de la esfera utilizando la malla 2 . ( $\left.h=55 \mathrm{~W} / \mathrm{m}^{2}{ }^{\circ} \mathrm{C}\right)$

Tabla 4-2: Máximos valores absolutos (norma infinito del error relativo porcentual) $\left(e \%=\left(\frac{T_{a}-T_{n}}{T_{a}}\right) \cdot 100\right)$ para distintas mallas generadas para un cilindro finito (para $\left.x=0\right) y$ tiempos de ejecución requeridos tiempo $\mathrm{cpu}_{\mathrm{cp}}(\mathrm{min})$.

\begin{tabular}{|c|c|c|c|c|}
\hline$\|\mathrm{e}\|_{\infty}(\%)$ & $r=0$ & $r=R / 2$ & $r=R$ & $\begin{array}{l}\text { tiempo } o_{\text {cpu }} \\
\text { (min) }\end{array}$ \\
\hline $\begin{array}{l}\text { Malla } 1 \\
392 \text { nodos, } 1609 \text { elementos }\end{array}$ & 4.15 & 1.99 & 8.23 & 0.71 \\
\hline $\begin{array}{l}\text { Malla } 2 \\
957 \text { nodos, } 4373 \text { elementos }\end{array}$ & 2.73 & 0.88 & 3.77 & 2.98 \\
\hline $\begin{array}{l}\text { Malla } 3 \\
2960 \text { nodos, } 14795 \text { elementos }\end{array}$ & 0.73 & 0.58 & 1.86 & 33.5 \\
\hline
\end{tabular}

A partir de la Tabla 4-2 se observa que la norma infinito del error decreció a medida que se aumentó el número de elementos de la malla, incrementando a su vez los esfuerzos computacionales. En este caso, la malla 
óptima sería una malla intermedia entre la 2 y 3.

\subsubsection{Validación del código computacional en una geometría 3D irregular mediante un software comercial de elementos finitos.}

El programa computacional fue también utilizado para simular la cocción de una pieza cárnea como la que se muestra en la Fig. 4-9, donde la sección transversal fue escaneada y digitalizada para simular la geometría irregular del dominio (Califano y Zaritzky, 1993). La malla generada se muestra en la Fig. 49 b) y fue obtenida a través del software COMSOL AB (2005), donde se exportó la información de la malla en la estructura "fem".

El dominio irregular del trozo cárneo presenta dos regiones diferentes (tejido muscular y graso) que tienen distintas propiedades térmicas como se puede visualizar en la Fig. 4-6 a). Las propiedades térmicas para el tejido muscular y para el tejido graso así como las condiciones de procesamiento se muestran en la Tabla 4-3. La pieza cárnea fue sumergida en un baño termostático a una temperatura uniforme de $70^{\circ} \mathrm{C}$, donde la temperatura inicial del producto fue $14.6^{\circ} \mathrm{C}$, la longitud total de la pieza fue de $17 \mathrm{~cm}$.

Tabla 4-3: Propiedades térmicas y condiciones de procesamiento.

\begin{tabular}{|c|c|}
\hline Propiedades Térmicas y Condiciones de proceso & \\
\hline \hline Temperatura inicial $\left({ }^{\circ} \mathrm{C}\right)$ & 14.6 \\
\hline Temperatura del fluido $\left({ }^{\circ} \mathrm{C}\right)$ & 70.0 \\
\hline Coeficiente de transferencia calórico $\mathrm{h}\left(\mathrm{W} / \mathrm{m}^{2}{ }^{\circ} \mathrm{C}\right)$ & 300.0 \\
\hline Tejido Muscular & 0.454 \\
\hline Conductividad térmica $\left(\mathrm{W} / \mathrm{m}^{\circ} \mathrm{C}\right)$ & 3477.80 \\
\hline Calor específico $\left(\mathrm{J} / \mathrm{kg}^{\circ} \mathrm{C}\right)$ & 969.2 \\
\hline Densidad $\left(\mathrm{kg} / \mathrm{m}^{3}\right)$ & \\
\hline Tejido graso & 0.175 \\
\hline Conductividad térmica $\left(\mathrm{W} / \mathrm{m}^{\circ} \mathrm{C}\right)$ & 4111.95 \\
\hline Calor específico $\left(\mathrm{J} / \mathrm{kg}^{\circ} \mathrm{C}\right)$ & 930.0 \\
\hline Densidad $\left(\mathrm{kg} / \mathrm{m}^{3}\right)$ & \\
\hline
\end{tabular}




\section{(F)}

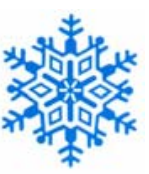

C I D C A
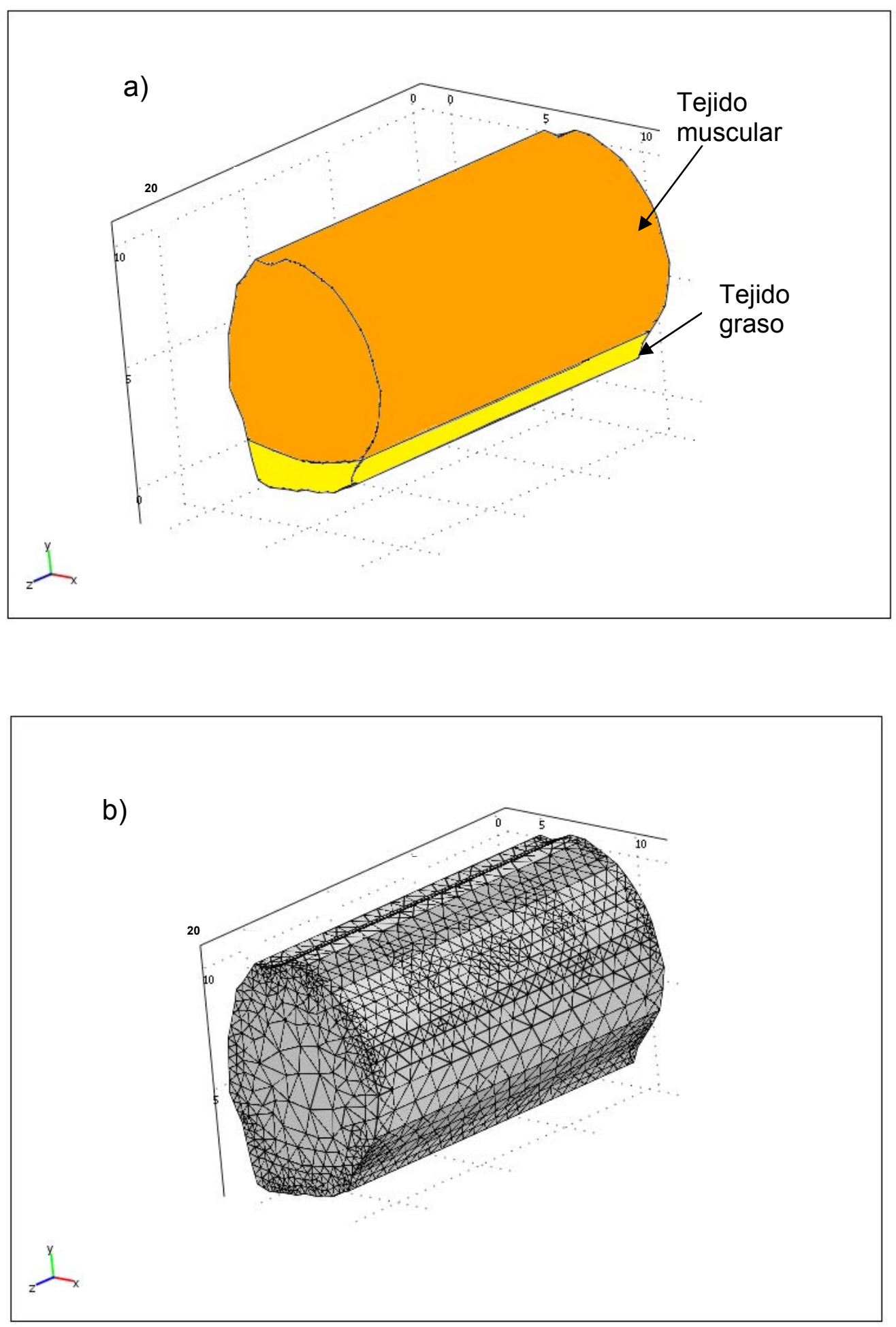

Fig. 4-9 a)Alimento cárneo compuesto por tejido muscular y tejido graso b) Malla utilizada para la simulación del alimento 
Las predicciones numéricas utilizando el código computacional en un punto central $(x=7.65 \mathrm{~cm} ., y=5.78 \mathrm{~cm}, z=3.5 \mathrm{~cm})$ y en un borde $(x=3.84 \mathrm{~cm}$, $y=7.56 \mathrm{~cm}, z=3.5 \mathrm{~cm}$ ) fueron comparadas con las predicciones de temperaturas obtenidas mediante el software comercial de simulación COMSOL AB (2005) y con resultados experimentales (ver Fig. 4-10).

Como puede observarse las predicciones numéricas obtenidas mediante el código propio presentan una buena concordancia con los resultados experimentales y con las predicciones numéricas obtenidas mediante un software comercial.

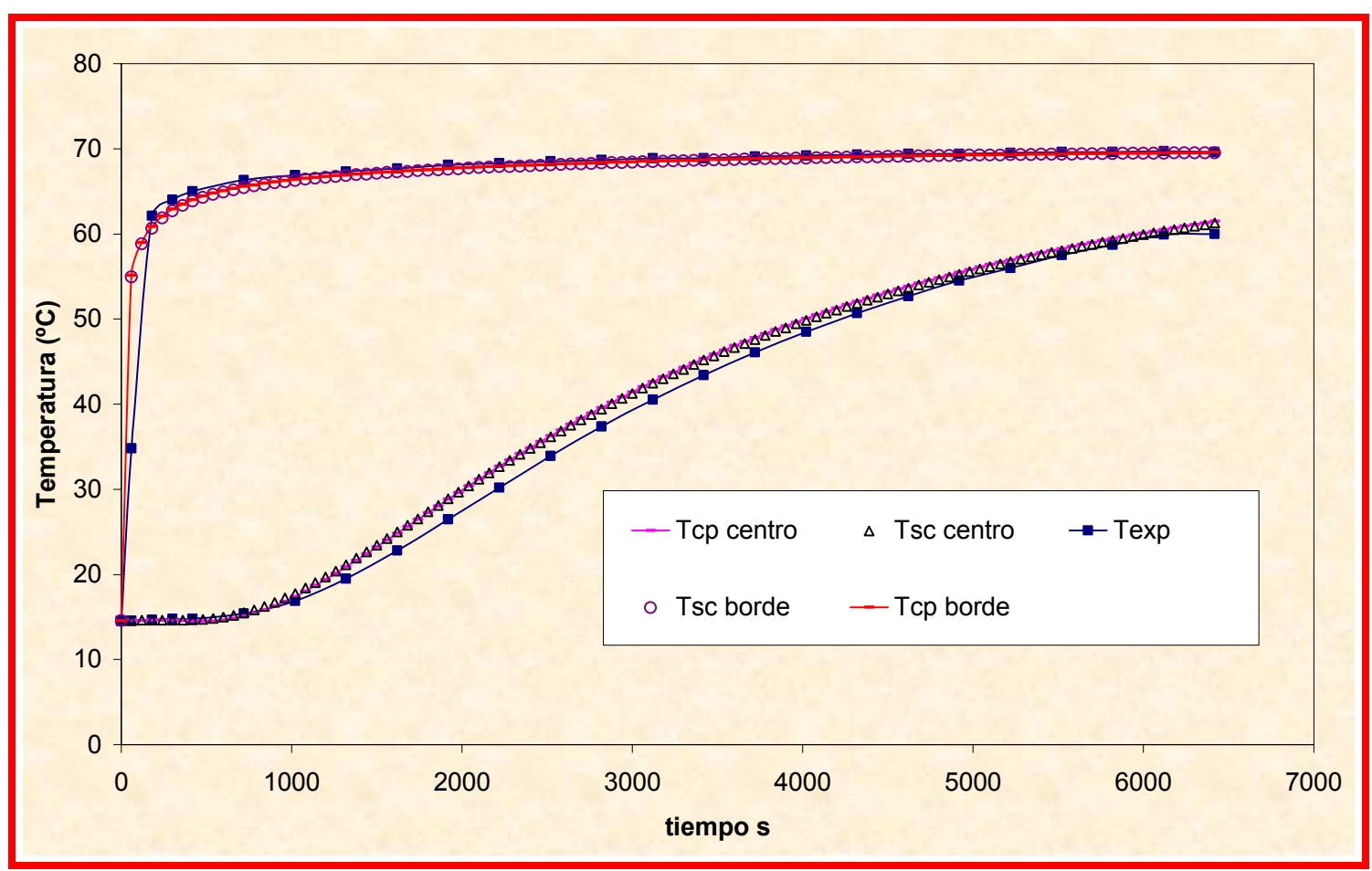

Fig. 4-10 Predicciones numéricas de temperatura en un punto central y borde durante el calentamiento de una pieza cárnea de geometría irregular 3D, utilizando el código propio desarrollado en la presente Tesis (Tcp), y un software comercial (Tsc); comparación con las temperaturas experimentales - $\square$ - 


\section{(a)}

C I D C A

\subsection{Conclusiones}

Se desarrolló un código computacional propio utilizando el MEF que resuelve el problema de transferencia de calor con condición de borde convectiva en una geometría tridimensional.

Se elaboraron subrutinas de pre- y post-procesamiento que permiten integrar la información de la malla con el programa principal y obtener en caso de ser requerido la temperatura en cualquier punto del dominio, la temperatura media, y flujo de calor en el dominio.

El código computacional fue validado comparando las temperaturas predichas con las soluciones analíticas en sistema de geometrías regulares (esfera y cilindro finito). El programa fue luego utilizado para simular la cocción en un producto con distintas propiedades térmicas y de geometría irregular. Las predicciones numéricas concordaron satisfactoriamente con las predicciones numéricas obtenidas a través de un software comercial y con resultados experimentales de calentamiento.

El programa computacional puede ser aplicado en productos alimenticios de geometrías tridimensionales utilizando un generador de malla externo, con la ventaja de poder acoplar subrutinas de interés para el usuario, como cinéticas de inactivación microbiana, cinéticas de pérdida de calidad, temperatura de fluido externo variable, etc. Se presenta a continuación (Apéndice 4-1) el programa computacional propio escrito en lenguaje Matlab 6.5 . 


\section{Apéndice 4-1}

\section{Código Computacional 3D desarrollado en Matlab 6.5}

\subsubsection{Preprocesamiento}

clc

grilla=fem.mesh.p';

nodes $=$ fem.mesh.t;

borde=fem.mesh.e;

coordenadas=grilla';

for $k=1$ :length(borde2)

for $i=1: n 2$

if $\operatorname{nodes}(1, \mathrm{i})==\operatorname{borde} 2(1, \mathrm{k})$

if $\operatorname{nodes}(2, \mathrm{i})==\operatorname{borde} 2(2, \mathrm{k})$

if $\operatorname{nodes}(3, i)==\operatorname{borde} 2(3, k)$

elementoborde $(1, \mathrm{k})=1$;

elementoborde $(2, \mathrm{k})=1$;

elementoborde $(3, \mathrm{k})=1$;

elementoborde $(4, \mathrm{k})=0$;

elementoborde $(5, \mathrm{k})=\mathrm{i}$;

end

end

end

if $\operatorname{nodes}(1, \mathrm{i})==\operatorname{borde} 2(1, \mathrm{k})$

if $\operatorname{nodes}(3, \mathrm{i})==\operatorname{borde} 2(2, \mathrm{k})$

if $\operatorname{nodes}(2, \mathrm{i})==\operatorname{borde} 2(3, \mathrm{k})$

elementoborde $(1, \mathrm{k})=1$;

elementoborde $(2, \mathrm{k})=1$;

elementoborde $(3, \mathrm{k})=1$;

elementoborde $(4, \mathrm{k})=0$; 


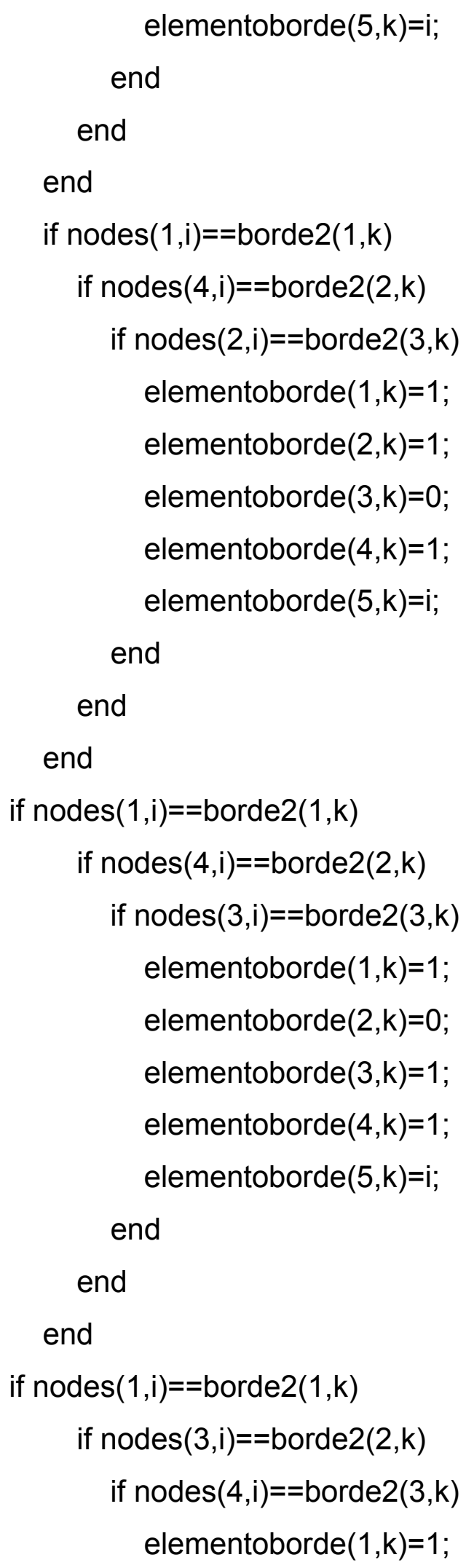




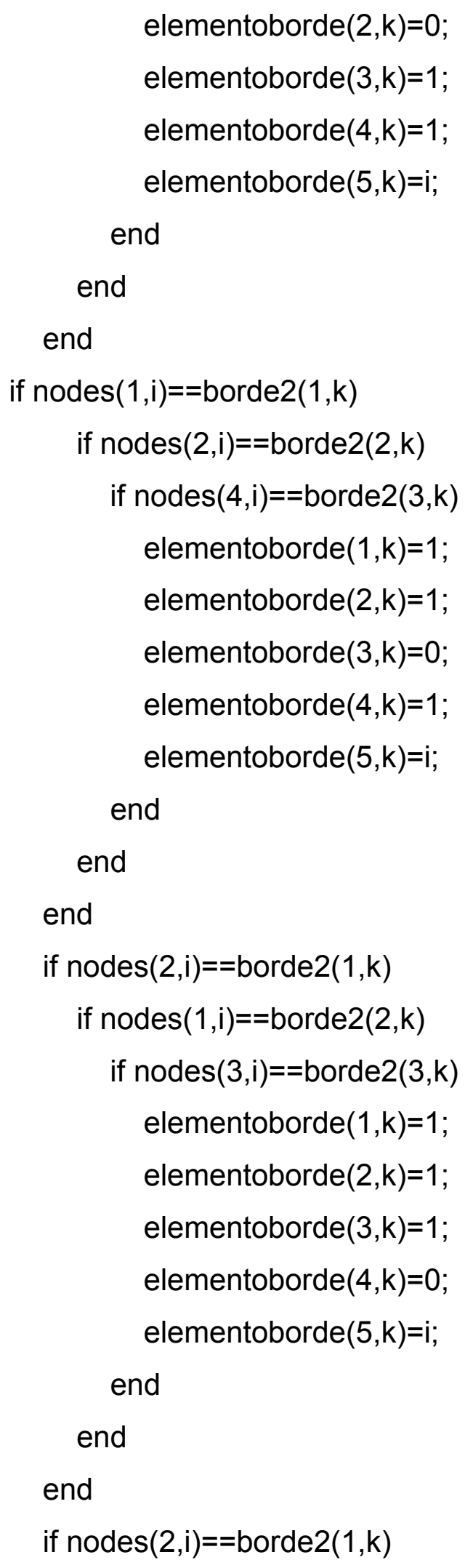




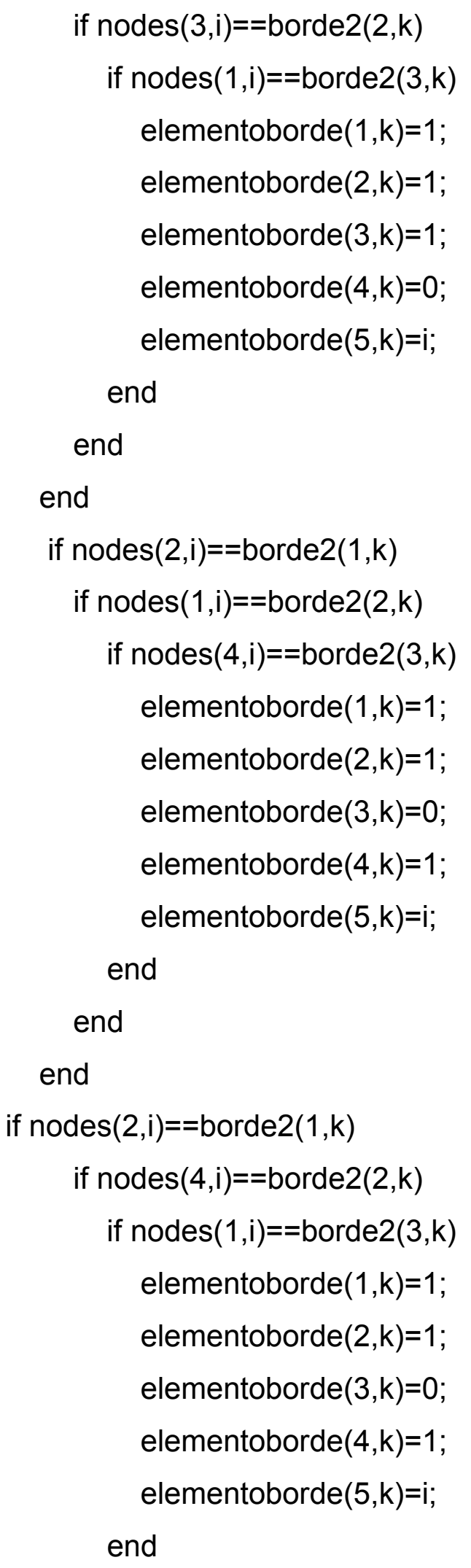




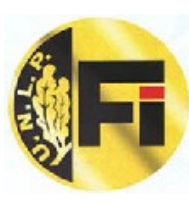

C I D C A

end

end

if nodes $(2, i)==\operatorname{borde} 2(1, k)$

if nodes $(4, \mathrm{i})==\operatorname{borde} 2(2, \mathrm{k})$

if $\operatorname{nodes}(3, i)==\operatorname{borde} 2(3, k)$

elementoborde $(1, \mathrm{k})=0$;

elementoborde $(2, \mathrm{k})=1$;

elementoborde $(3, \mathrm{k})=1$;

elementoborde $(4, \mathrm{k})=1$;

elementoborde $(5, \mathrm{k})=\mathrm{i}$;

end

end

end

if $\operatorname{nodes}(2, i)==\operatorname{borde} 2(1, \mathrm{k})$

if $\operatorname{nodes}(3, i)==\operatorname{borde} 2(2, \mathrm{k})$

if $\operatorname{nodes}(4, \mathrm{i})==\operatorname{borde} 2(3, \mathrm{k})$

elementoborde $(1, \mathrm{k})=0$;

elementoborde $(2, \mathrm{k})=1$;

elementoborde $(3, \mathrm{k})=1$;

elementoborde $(4, \mathrm{k})=1$;

elementoborde $(5, \mathrm{k})=\mathrm{i}$;

end

end

end

if $\operatorname{nodes}(3, i)==\operatorname{borde} 2(1, \mathrm{k})$

if $\operatorname{nodes}(1, \mathrm{i})==\operatorname{borde} 2(2, \mathrm{k})$

if $\operatorname{nodes}(2, \mathrm{i})==\operatorname{borde} 2(3, \mathrm{k})$

elementoborde $(1, \mathrm{k})=1$;

elementoborde $(2, \mathrm{k})=1$;

elementoborde $(3, \mathrm{k})=1$; 


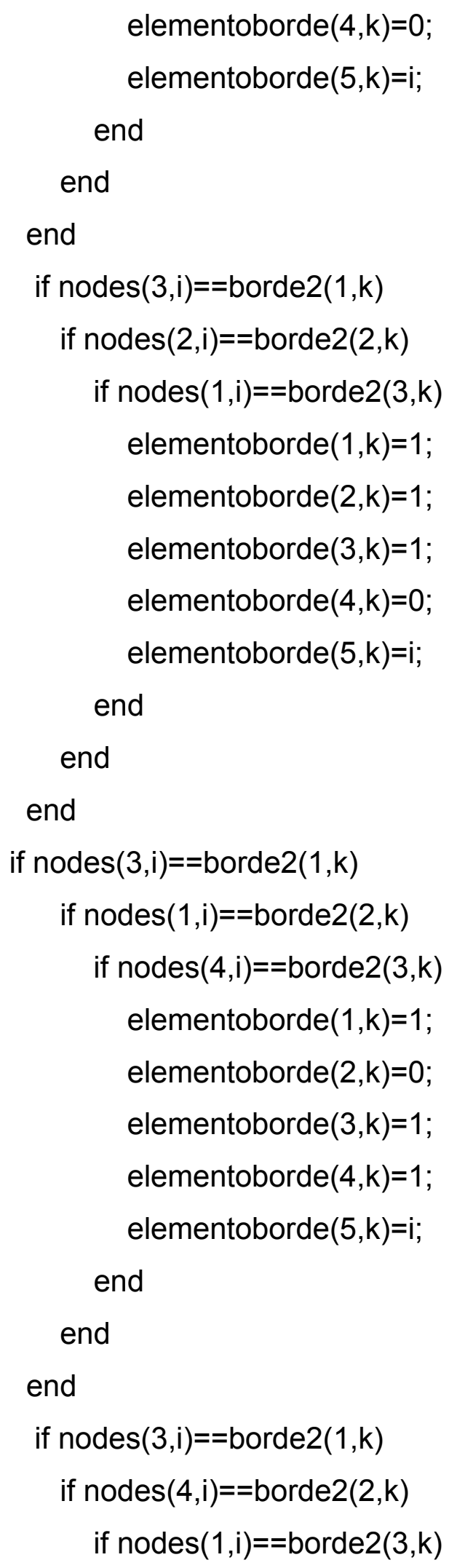




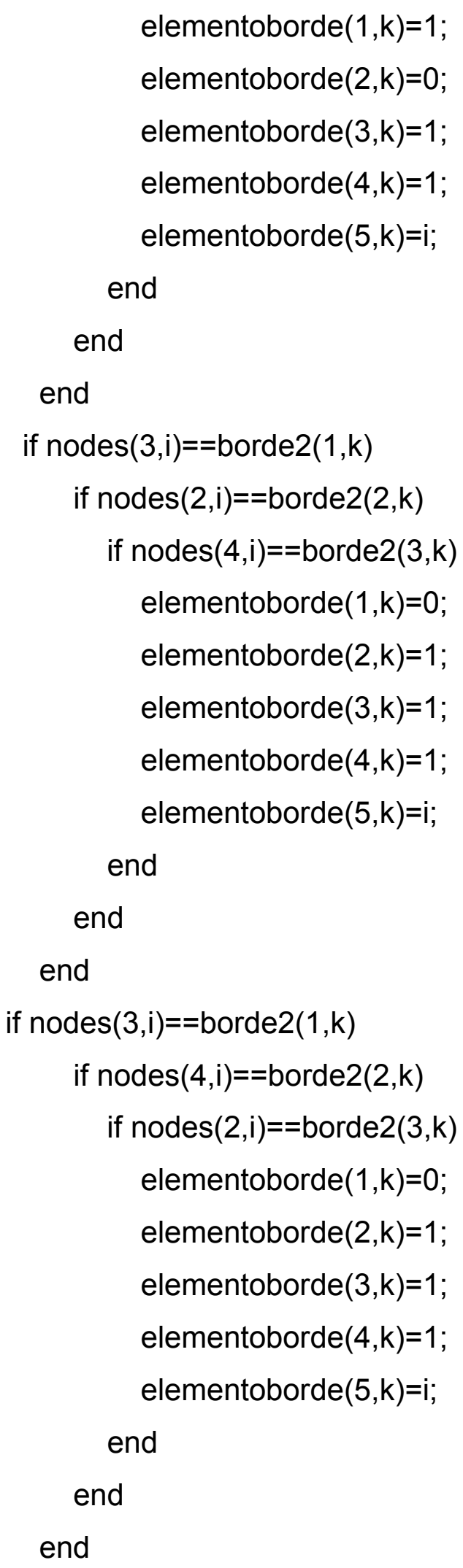




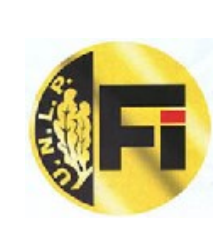

C I D C A

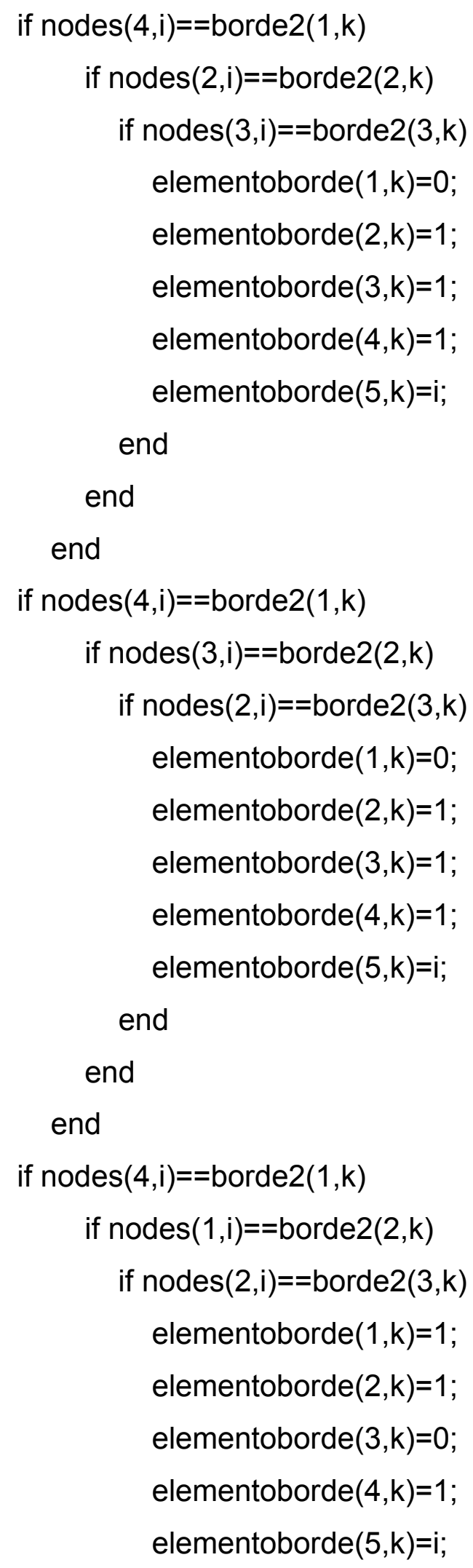




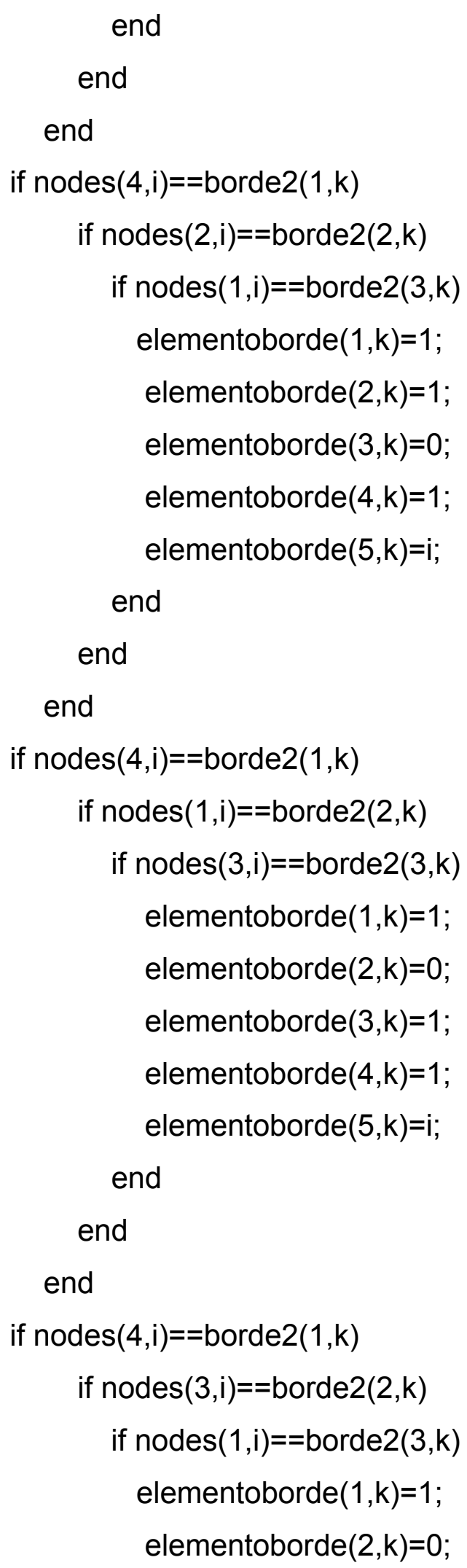


elementoborde $(3, \mathrm{k})=1$;

elementoborde $(4, \mathrm{k})=1$;

elementoborde $(5, \mathrm{k})=\mathrm{i}$;

end

end

end

end

end

save malla.mat

\subsubsection{Programa principal}

clear all,clc

load malla.mat;

$\% \%$ numero de nodos totales

nodos_t=length(grilla);

$\%$ numero de elementos

n_elem=length(nodes);

\%numero de nodos por elemento

$\mathrm{nn}$ _elem $=4$;

$\%$ grados de libertad por elemento

gr_l=1;

alfa=1;

Volumen=0;

AreaAnalitica $=0$;

AreaNumericaTot $=0$;

$K G=$ sparse(zeros(nodos_t));

$\mathrm{CG}=$ sparse(zeros(nodos_t));

$\mathrm{FG}=$ sparse(zeros(nodos_t,1));

$\mathrm{T}=$ ones(nodos_t, 1$)$;

$\mathrm{Ti}=17.2$;

$\mathrm{T}=\mathrm{Ti}^{*} \mathrm{~T}$; 


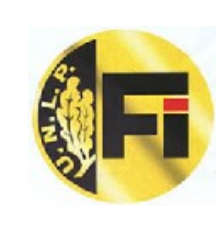

C I D C A

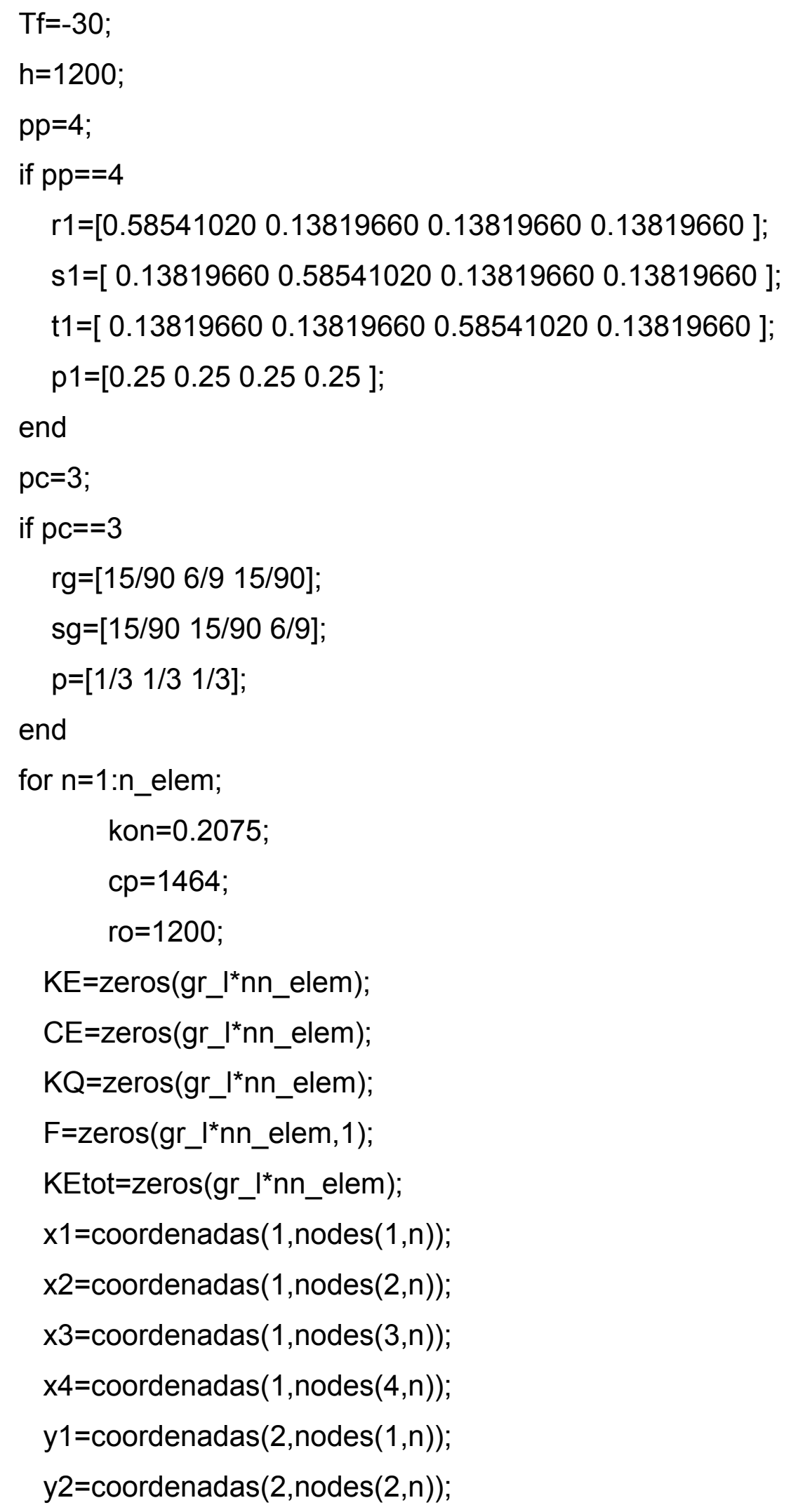




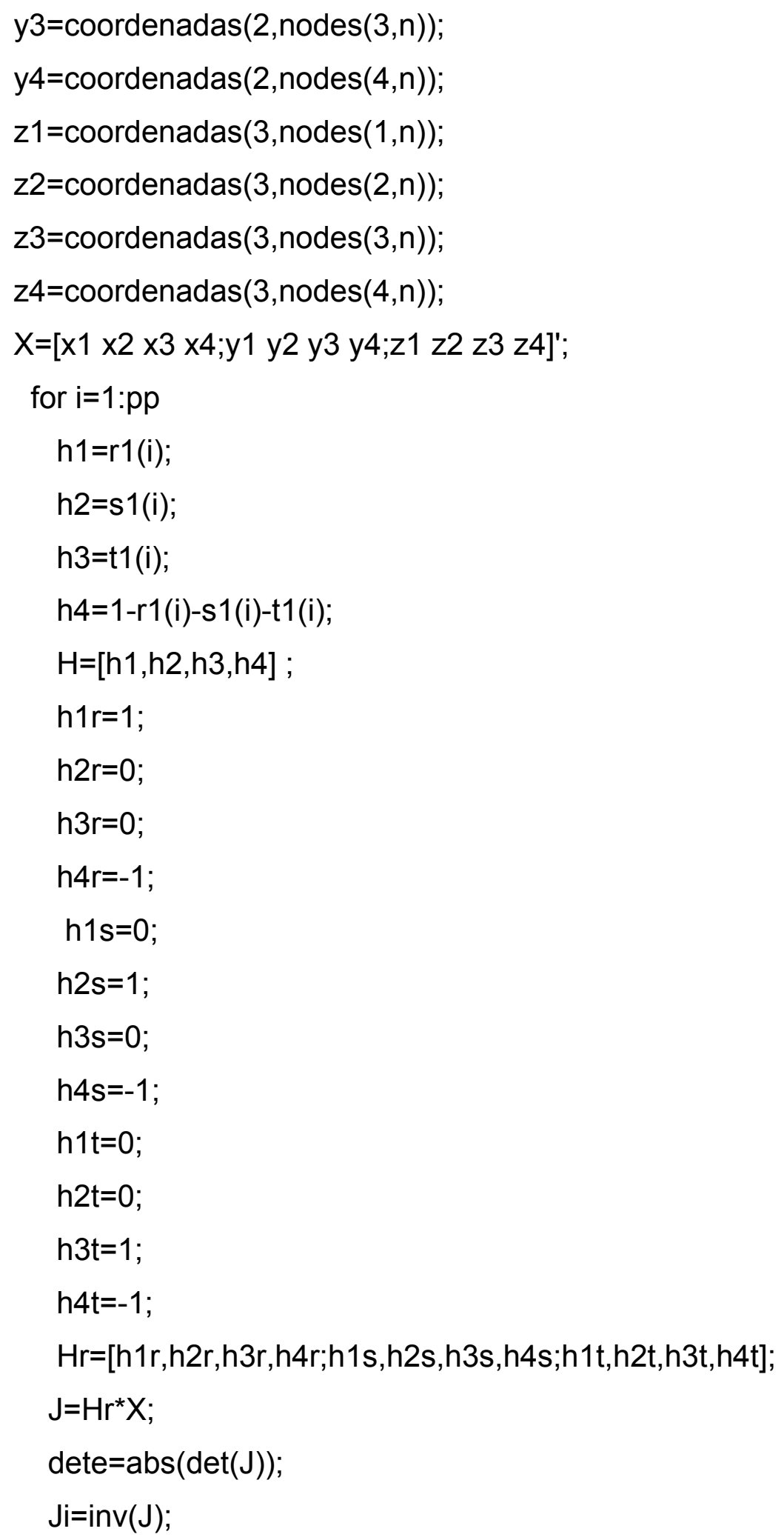


$\mathrm{BE}=\mathrm{Ji}{ }^{*} \mathrm{Hr}$

w1=p1(i);

$\mathrm{KE}=\mathrm{KE}+1 / 6^{*}$ dete $^{*} \mathrm{w} 1^{*} \mathrm{BE}{ }^{* *} \mathrm{kon}{ }^{*} \mathrm{BE}$;

$\mathrm{CE}=\mathrm{CE}+1 / 6^{*}$ dete $^{*} \mathrm{w} 1^{*} \mathrm{H}^{* *} \mathrm{H}^{*} \mathrm{ro}{ }^{*} \mathrm{cp}$;

Volumen=Volumen $+\operatorname{dete}^{*} \mathrm{w} 1 / 6$;

end

for $m=1$ :length(elementoborde)

if $\mathrm{n}==$ elementoborde $(5, \mathrm{~m})$

Te=Tf*ones(nn_elem,1);

if elementoborde $(1, m)==0$

Area $=0$;

$\mathrm{c} 1=(\mathrm{y} 3-\mathrm{y} 4)^{\star}(\mathrm{z} 2-\mathrm{z} 4)-(\mathrm{z} 3-\mathrm{z} 4)^{\star}(\mathrm{y} 2-\mathrm{y} 4)$;

$\mathrm{c} 2=(\mathrm{z} 3-\mathrm{z} 4)^{*}(\mathrm{x} 2-\mathrm{x} 4)-(\mathrm{x} 3-\mathrm{x} 4)^{*}(\mathrm{z} 2-\mathrm{z} 4)$;

$\mathrm{c} 3=(\mathrm{x} 3-\mathrm{x} 4)^{*}(\mathrm{y} 2-\mathrm{y} 4)-(\mathrm{y} 3-\mathrm{y} 4)^{*}(\mathrm{x} 2-\mathrm{x} 4)$;

AreaAnalitica $=$ AreaAnalitica $+0.5^{\star} \operatorname{sqrt}\left(c 1^{\wedge} 2+c 2^{\wedge} 2+c 3^{\wedge} 2\right)$;

AreaAnaUno $=0.5^{\star} \operatorname{sqrt}\left(\mathrm{c} 1^{\wedge} 2+\mathrm{c} 2^{\wedge} 2+\mathrm{c} 3^{\wedge} 2\right)$;

$\mathrm{c}=[\mathrm{c} 1 \mathrm{c} 2 \mathrm{c} 3]$;

$[$ valor, imax $]=\max (\operatorname{abs}(c))$;

$\mathrm{h} 2 \mathrm{t}=0$;

$h 3 t=1$;

$\mathrm{h} 4 \mathrm{t}=-1$;

$h 2 s=1$;

$h 3 s=0$;

$\mathrm{h} 4 \mathrm{~s}=-1$;

if imax $==1$

aux $=\operatorname{sqrt}\left(1+(\mathrm{c} 2 / \mathrm{c} 1)^{\wedge} 2+(\mathrm{c} 3 / \mathrm{c} 1)^{\wedge} 2\right)$;

$d z t=z 4^{*} h 4 t+z 2 * h 2 t+z 3^{*} h 3 t ;$

$d z s=z 4 * h 4 s+z 2 * h 2 s+z 3 * h 3 s$;

$d y t=y 4 * h 4 t+y 2 * h 2 t+y 3{ }^{*} h 3 t ;$

dys $=y 4 * h 4 s+y 2 * h 2 s+y 3 * h 3 s$; 


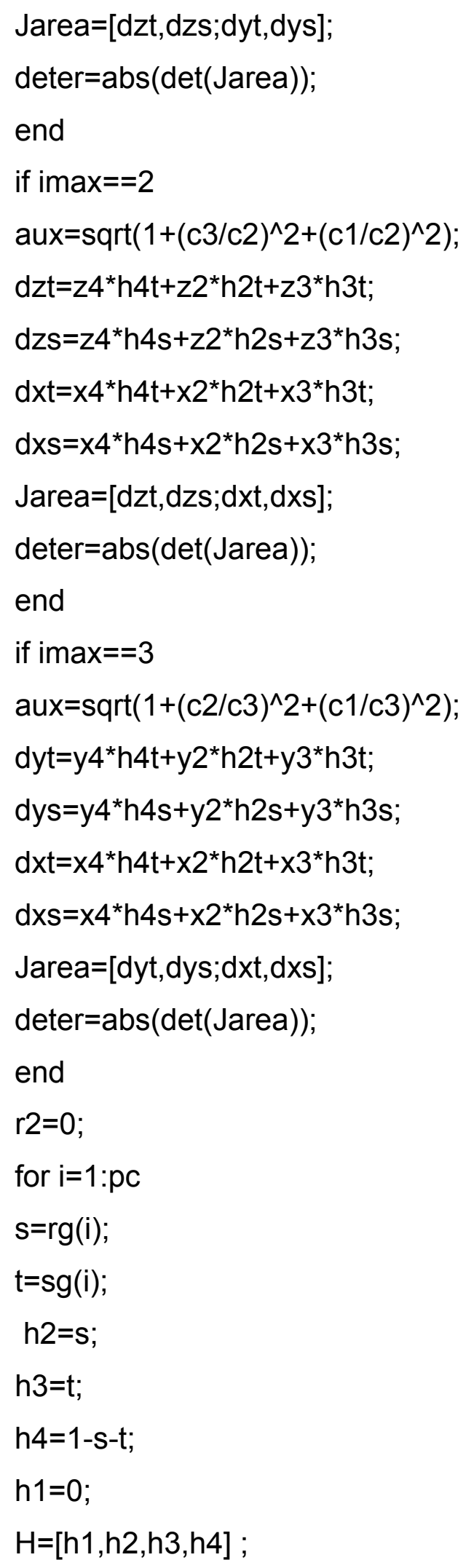




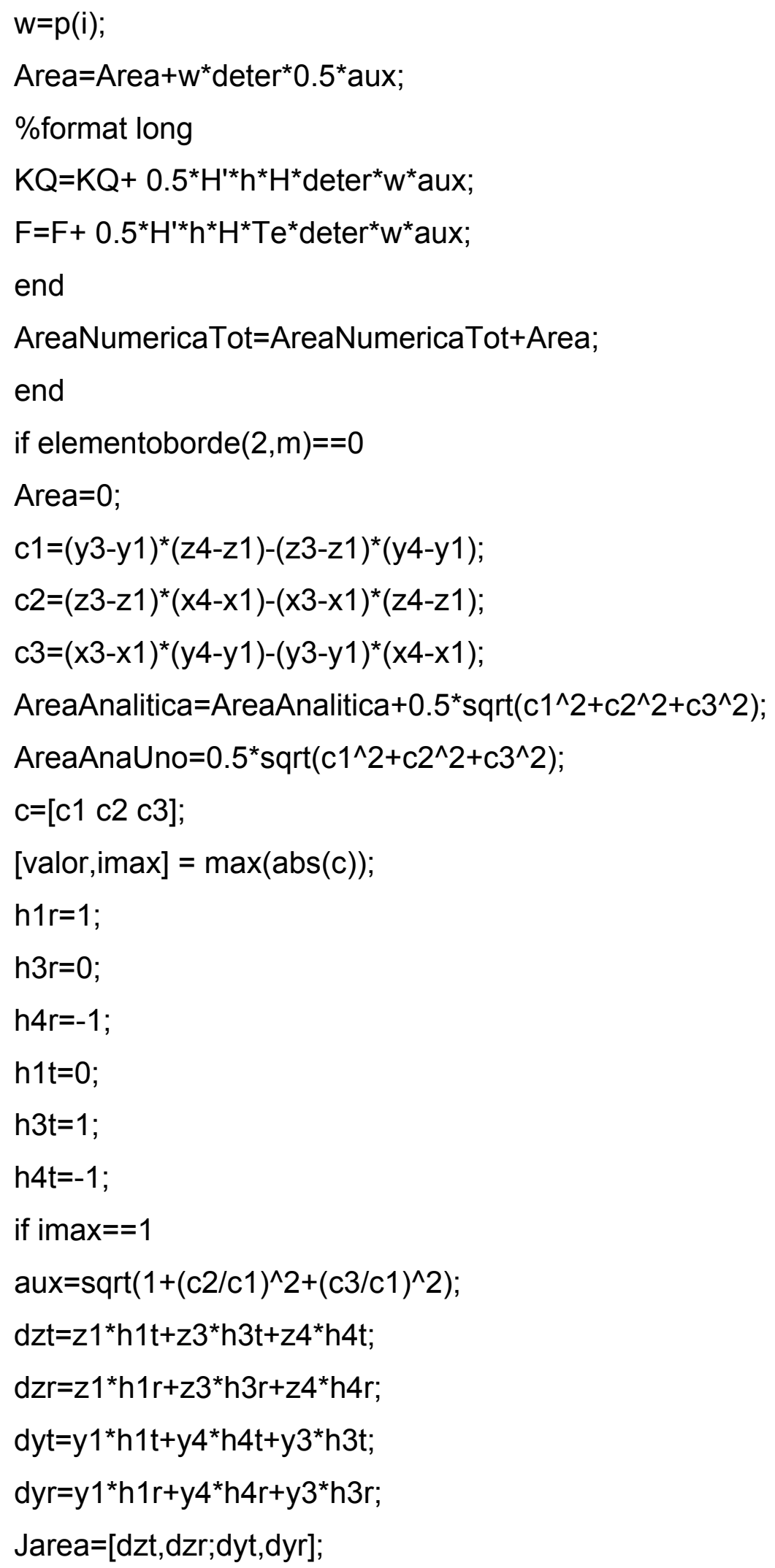




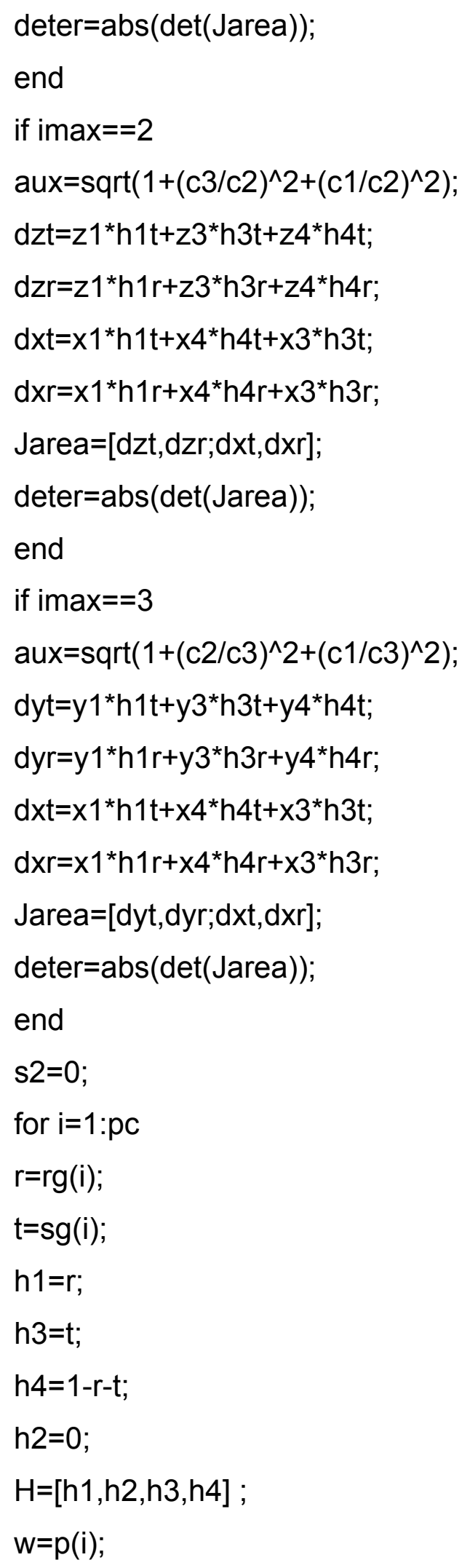




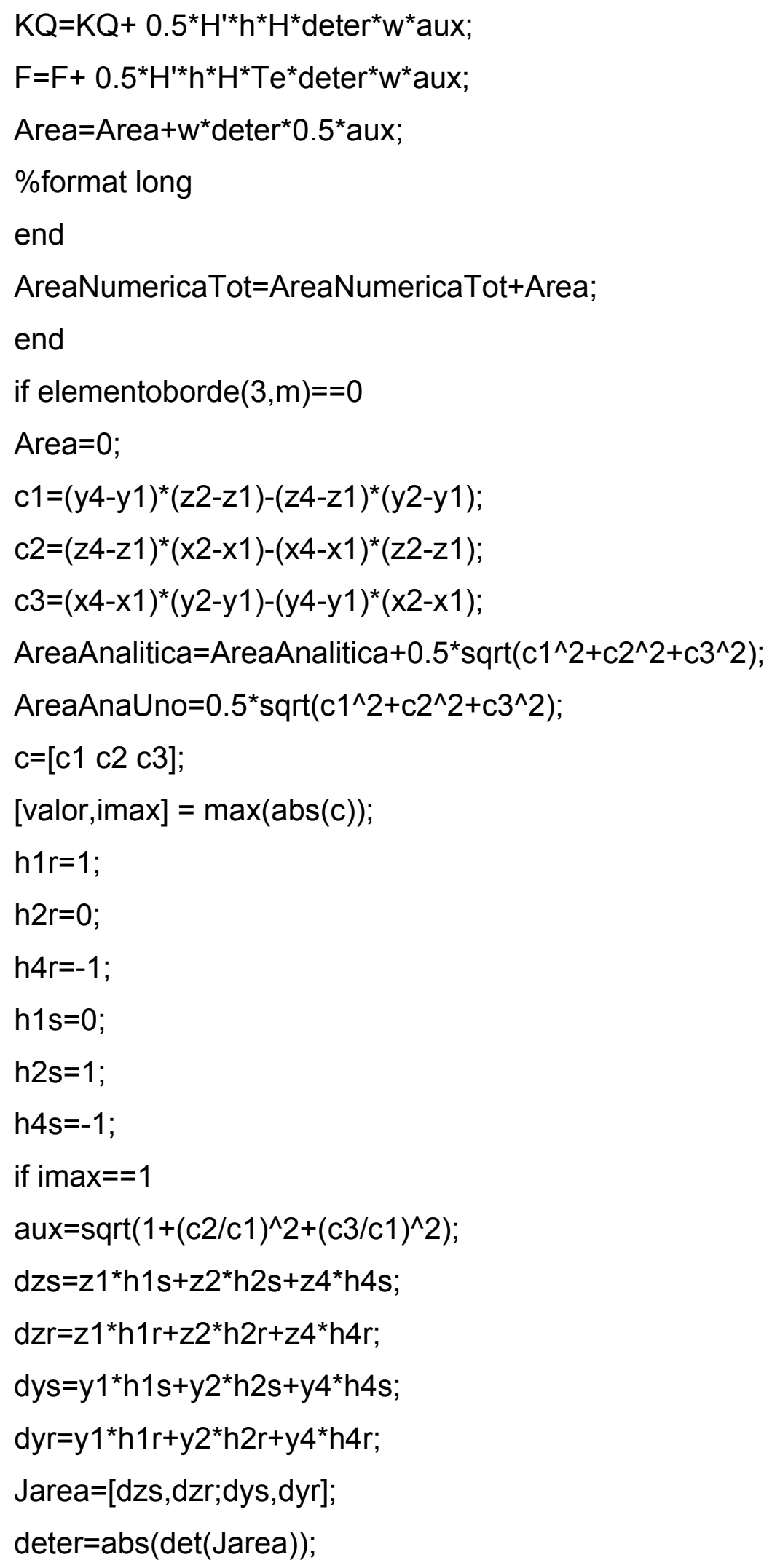




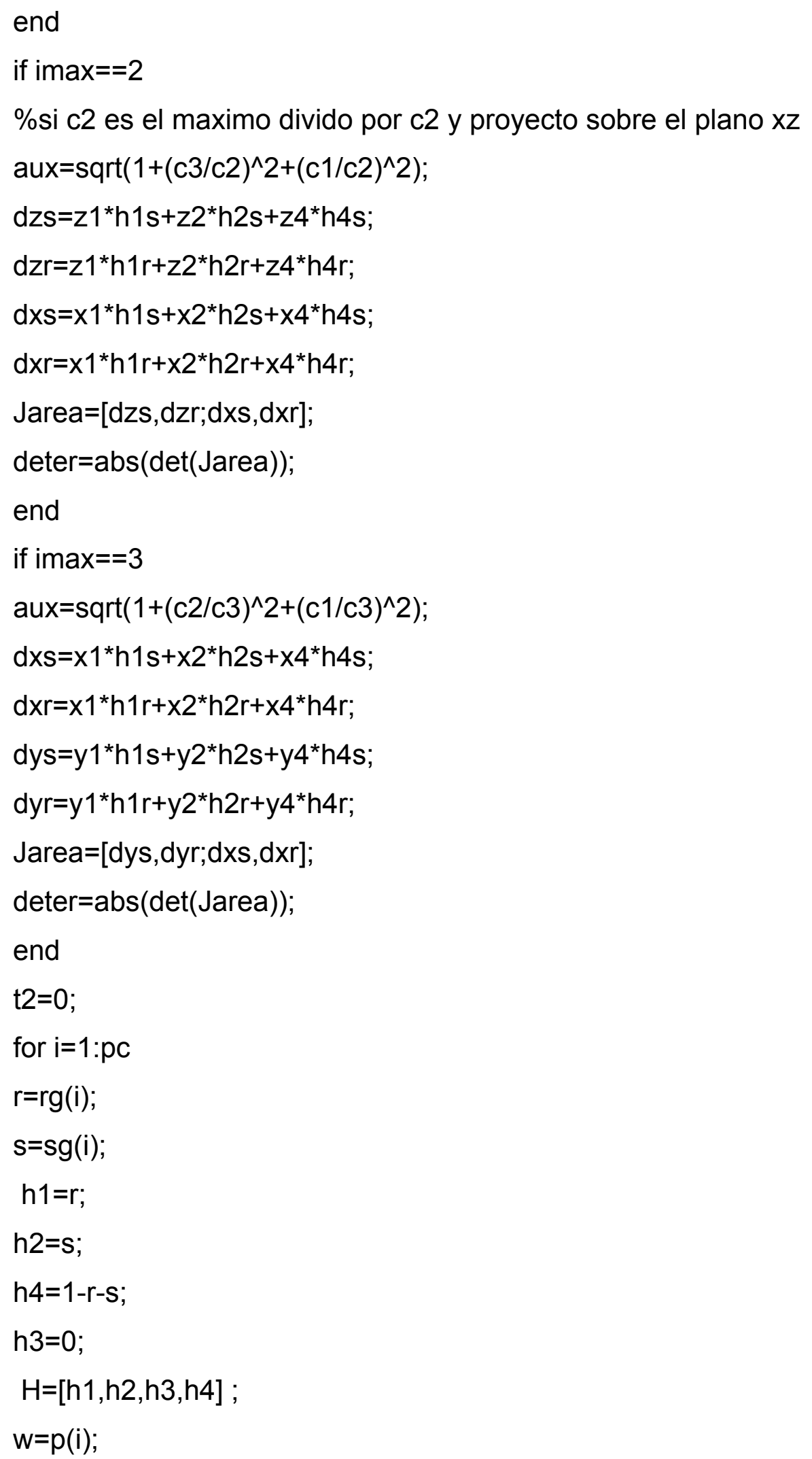




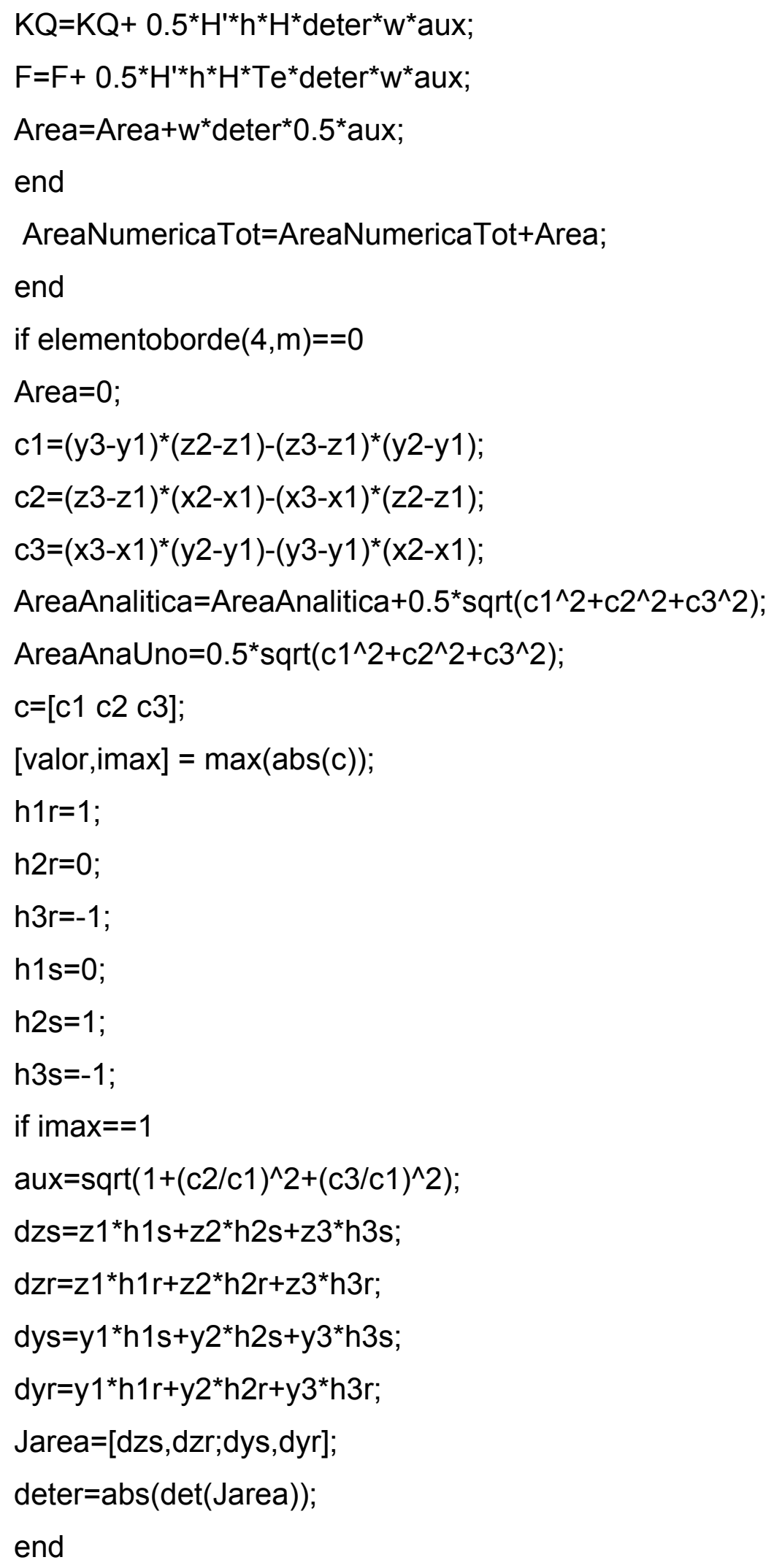




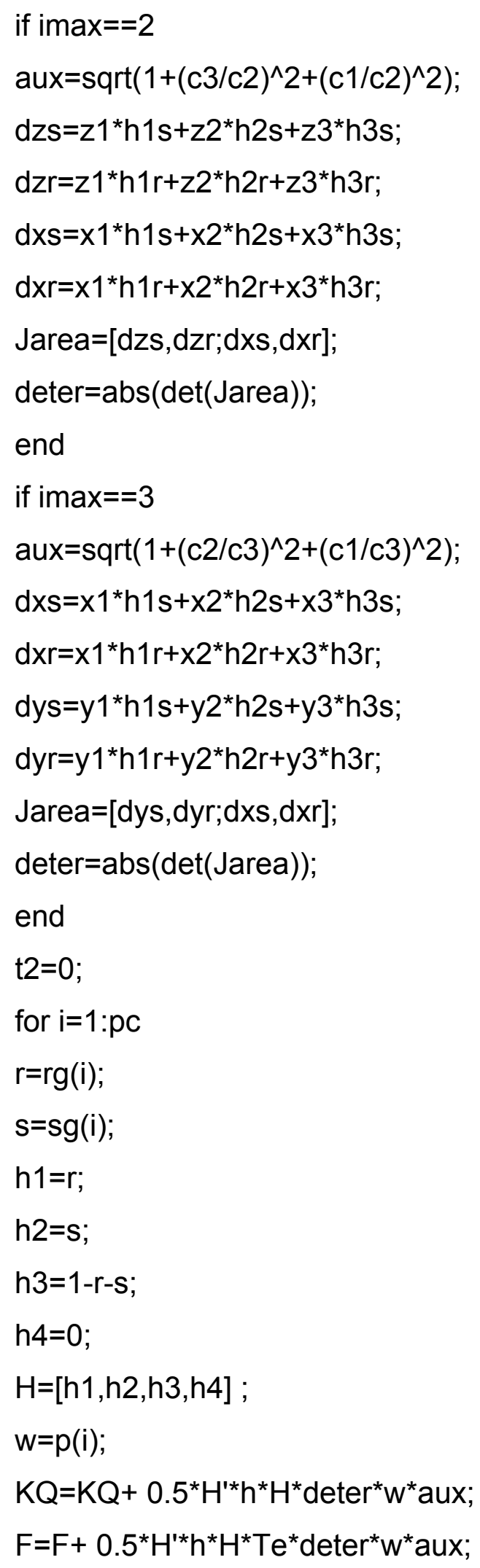


Area $=$ Area $+w^{*}$ deter ${ }^{*} 0.5^{*}$ aux;

end

AreaNumericaTot=AreaNumericaTot+Area;

end

end

end

KEtot=KQ+KE;

for i=1:gr_I*nn_elem

for $j=1: g r \_{ }^{*} n n \_$elem

i_g=nodes $(\mathrm{i}, \mathrm{n})$;

j_g=nodes $(j, n)$;

$K G\left(i \_g, j \_g\right)=K G\left(i \_g, j \_g\right)+K E t o t(i, j)$;

$C G\left(i \_g, j \_g\right)=C G\left(i \_g, j \_g\right)+C E(i, j)$;

end

$F G\left(i \_g, 1\right)=F G\left(i \_g, 1\right)+F(i, 1)$;

end

end

$K G=$ sparse $(K G)$;

$F G=$ sparse $(F G)$;

$\mathrm{CG}=$ sparse $(C G)$;

tcpu $1=($ cputime-tcpu0 $) / 60$

tcpu $0=$ cputime;

tiempof $=600$;

deltatiempo $=0.5$;

nciclo $=1$

for $\mathrm{i}=1$ :tiempof/deltatiempo

tciclo $=$ deltatiempo ${ }^{*}(\mathrm{i}) / 60$;

$D=\left(\right.$ deltatiempo* $\left.(1-\text { alfa })^{*} F G\right)+($ alfa*deltatiempo* $F G)+(C G-((1-$

alfa) ${ }^{*}$ deltatiempo*KG) $)^{*} \mathrm{~T}$;

$B=C G+a l f a *$ deltatiempo*KG; 
$\mathrm{Tn}=\mathrm{B} \backslash \mathrm{D}$;

$\mathrm{T}=\mathrm{Tn}$;

if tciclo $==10^{*}$ nciclo

$[\mathrm{Tmin}, \mathrm{imin}]=\min (\mathrm{T})$;

nodomin=imin;

tpunto (nciclo)=tciclo;

[Tpromedio]=Tmedia3D(n_elem,nodes, T,coordenadas,pp,p1,r1,s1,t1,tciclo);

Tmedia(nciclo, 1)=Tpromedio;

longx $=0.02$;

ndiv=2;

for $\mathrm{i}=1:$ ndiv +1

punto $=(\mathrm{i}-1)^{*}$ long $\mathrm{x} / \mathrm{ndiv}$;

$x=16.0524 \mathrm{E}-3$;

$y=6.8176 \mathrm{E}-3$;

$\mathrm{z}=41 \mathrm{E}-3$;

[h1,h2,h3,h4, nodo1, nodo2, nodo3, nodo4]=pospro3D(x,y,z,grilla,nodes);

Tpunto $($ nciclo, 1$)=\mathrm{T}(\text { nodo } 1)^{*} \mathrm{~h} 1+\mathrm{T}(\text { nodo } 2)^{*} \mathrm{~h} 2+\mathrm{T}(\text { nodo3 })^{*} \mathrm{~h} 3+\mathrm{T}(\text { nodo } 4)^{*} \mathrm{~h} 4$

end

else

continue

end

nciclo $=$ nciclo +1

tcpu2=(cputime-tcpu0)/60

end

tcpu3=(cputime-tcpu0)/60 
Capítulo 5 


\section{Simulación del proceso de refrigeración de alimentos con composición heterogénea de geometría irregular 3D utilizando el MEF}

\subsection{Introducción}

La refrigeración es uno de los tratamientos industriales de conservación de alimentos más utilizado. La ventaja radica en que la actividad de los microorganismos y de las enzimas de los alimentos se ve disminuida, con el consiguiente retraso en la degradación de sus componentes; en consecuencia los alimentos presentan una mayor vida útil.

El sector productivo dedicado a la elaboración de alimentos semiterminados recurre al proceso de refrigeración para mantener o transportar sus productos a los establecimientos de venta al público. Específicamente el sector industrial dedicado a la elaboración de empanadas, centraliza su proceso de elaboración en una planta donde posteriormente son enfriados y/o congelados para su transporte y distribución. En los establecimientos estos productos son horneados de acuerdo a la demanda ofreciendo a los consumidores un producto fresco "recién horneado".

Se identificó que este tipo de producto semi-elaborado es de marcado interés para el sector industrial, y por lo tanto surgió la inquietud de conocer los tiempos de refrigeración para distintas condiciones, ya sea en cámaras o túnel, variando a su vez las temperaturas del medio refrigerante.

Un aspecto que merece resaltarse acerca de algunos de estos productos pre-elaboradores es que está compuesto por regiones, con propiedades termofísicas y químicas diferentes (por ejemplo masa cruda y relleno en el caso empanadas), lo cual influye directamente en los tiempos de refrigeración.

La simulación numérica del proceso de refrigeración permitiría establecer condiciones óptimas de operación y/o definir las características y 
requerimientos de distintos coeficientes de transferencia de calor en la interfase (h) en los equipos de enfriamiento al momento de decidir la adquisición de instalaciones industriales.

Los objetivos del presente Capítulo son:

Desarrollar un modelo matemático y el correspondiente código computacional que simule el proceso de enfriamiento de un producto compuesto por más de un material teniendo en cuenta la geometría irregular tridimensional del sistema.

Medir experimentalmente para cada material (relleno cárnico y masa farinácea externa) la densidad y el calor específico utilizando Calorimetría Diferencial de Barrido (DSC).

Validar el modelo mediante experimentos de refrigeración en cámara.

Utilizar el modelo para predecir tiempos de refrigeración variando distintos parámetros y condiciones del proceso, tales como el coeficiente de transferencia de calor, la temperatura del aire refrigerante y la temperatura inicial de producto.

\subsection{Materiales y métodos}

\subsubsection{Formulación matemática}

El balance microscópico diferencial de energía con propiedades constantes en tres dimensiones con condición de borde convectiva se resolvió numéricamente mediante MEF utilizando el procedimiento descripto en el Capítulo anterior.

\subsubsection{Optimización del código computacional}

El código computacional implementando el MEF se programó en Matlab 6.5. En virtud de mejorar los tiempos de ejecución de máquina se realizaron optimizaciones en el código computacional, tal como fue descripto detallada en los Capítulos 2 y 4. 
> Se utilizó una función dentro del Matlab llamada "sparse", la cual convierte a las matrices CG, KG y al vector FG en arreglos compactos donde sólo se almacenan los elementos distintos de cero.

Para calcular el aporte proveniente de las condiciones de borde se independizó el lazo de los elementos del dominio, del lazo de los elementos de borde. De esta manera el aporte proveniente de un elemento que se encuentra bajo la condición de borde convectiva se ensambla dentro de las matrices y vectores globales en forma separada. Se utilizaron además las funciones de Matlab "tsearchn" y "dsearchn" que permiten identificar los índices de elementos y nodos en las matrices que forman la estructura de la malla para agilizar la búsqueda de puntos de interés en la misma.

$>$ El sistema de ecuaciones diferenciales ordinarias se resolvió implementando la subrutina "ODE", incorporada en el Matlab, la cual permite resolver el problema variando el incremento en el tiempo de acuerdo a una medida del error (Shampine, 2004). Esta subrutina, donde el incremento del tiempo es ajustable en las diferentes iteraciones, permite disminuir significativamente los tiempos de ejecución de máquina en comparación con los métodos que utilizan incrementos fijos del tiempo.

\subsubsection{Sistema experimental para validar el modelo numérico}

El producto cárneo-farináceo de geometría irregular (empanadas) se elaboró utilizando carne picada magra previamente cocida y círculos de masa (2 $\mathrm{mm}$ de espesor).

Con el objeto de generar productos que mantuvieran en todos los casos la misma geometría, se utilizó un molde plástico específicamente diseñado para la elaboración de empanadas.

Para la refrigeración se utilizó una cámara a una temperatura media de 5 ${ }^{\circ} \mathrm{C}$ donde se ubicó el producto, registrando la temperatura en función del tiempo en varios puntos del alimento y en el medio exterior por medio de termocuplas cobre constantán conectadas a un adquisidor de datos (Testo 175, 
Testo AG, Alemania). Las historias térmicas obtenidas a través de los experimentos realizados en cámaras se utilizaron para validar el modelo numérico desarrollado.

\subsubsection{Generación de la malla}

Para obtener la geometría real del producto se realizaron prototipos en parafina, utilizando el mismo molde plástico previamente descripto para elaboración del producto alimenticio. Estos prototipos fueron luego utilizados para la representación espacial tridimensional del objeto de geometría irregular. La geometría se obtuvo a través de archivos CAD generados mediante el software Solidworks.

Los archivos CAD fueron luego importados al mallador (COMSOL AB, 2005) para su discretización espacial en elementos tetraédricos.

\subsubsection{Determinación experimental de las propiedades termofísicas del producto (calor específico aparente y densidad).}

El cálculo del calor especifico $\mathrm{Cp}$ se realizó a través de un Calorímetro Diferencial de Barrido (DSC), TA Instruments, New Castle, Delaware, USA modelo Q100 controlado por un módulo TA 5000 que tiene un sistema de enfriamiento rápido ("quenching") bajo una atmósfera de Nitrógeno a $20 \mathrm{ml} / \mathrm{min}$. En este equipo se hicieron corridas de calentamiento a una velocidad de $2^{\circ} \mathrm{C} / \mathrm{min}$ desde $-50^{\circ} \mathrm{C}$ hasta $100{ }^{\circ} \mathrm{C}$ con un período isotérmico inicial de 10 minutos a $-50^{\circ} \mathrm{C}$. La primera corrida se realizó para obtener una línea de base por lo que se utilizaron dos cápsulas vacías de igual peso. Luego se obtuvo el termograma correspondiente a un estándar de zafiro, la tercera corrida se realizó con la muestra de masa cruda y la cuarta con carne picada cocida. El procedimiento completo se realizó por triplicado. El equipo fue previamente calibrado y la temperatura corregida con dos estándares, indio y agua bidestilada. El cálculo del Cp se realizó según la norma ASTM E1269 y 
McNaughton y Mortimer (1975) tomando el promedio entre los replicados. A modo de ejemplo la Fig. 5-1 muestra esquemáticamente un termograma obtenido mediante DSC.

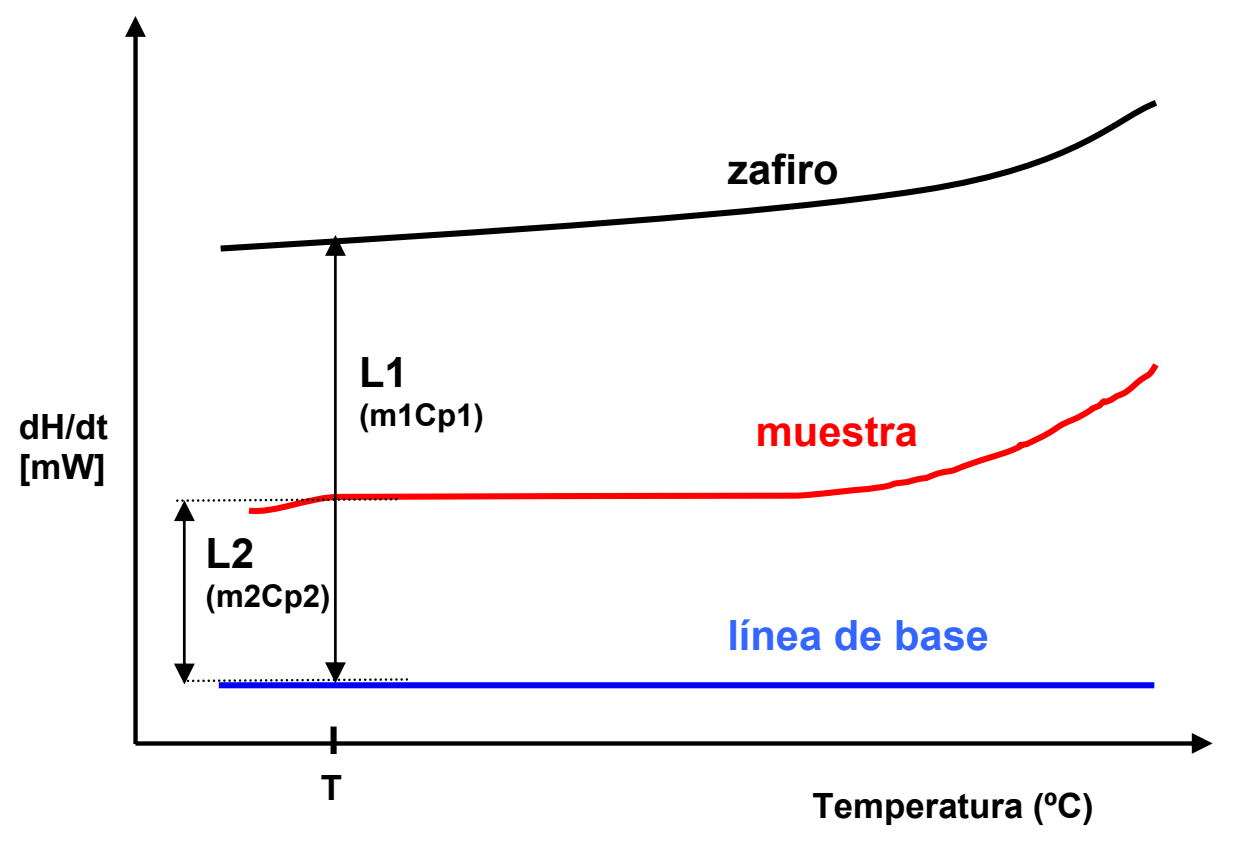

Fig. 5-1 Termograma obtenido mediante DSC

El cálculo del Cp se obtiene a partir de las alturas L1 y L2 conociendo el peso de la muestra (m2) y del zafiro (m1) mediante la siguiente ecuación:

$$
\frac{\mathrm{Cp} 2}{\mathrm{Cp} 1}=\frac{\mathrm{L} 2}{\mathrm{~L} 1} \cdot \frac{\mathrm{m} 1}{\mathrm{~m} 2}
$$

El contenido de agua de cada material, carne y masa, se determinó colocando las cápsulas en estufa de vacío a $80^{\circ} \mathrm{C}$ hasta obtener el peso seco constante entre dos medidas consecutivas.

La densidad del producto (masa/volumen) se determinó utilizando el método descripto por Baik y col (2001) donde una muestra de cada material se corta generando una geometría regular, cuyo volumen se puede calcular a 
partir de las dimensiones de la misma medidas con calibre. La masa de dicha muestra se midió mediante una balanza analítica y la densidad se calcula a partir del cociente entre la masa y el volumen de la muestra. También se utilizó el método de las semillas (en inglés "Seed replacement method") para obtener el volumen irregular de la empanada; en este método se conoce el volumen inicial de las semillas, luego se coloca la muestra midiendo por desplazamiento el volumen de la muestra más las semillas, y por diferencia se obtiene el volumen del producto.

\subsubsection{Determinación experimental del coeficiente de transferencia de calor en la interfase (h)}

Para estimar el coeficiente de transferencia de calor se aplicó el Método Transiente de determinación del $\mathrm{h}$, en donde existe un gradiente de temperaturas dentro del sólido (Rahman, 1995). Cuando la geometría es irregular y/o existen condiciones de borde variables, no se pueden aplicar soluciones analíticas para estimar el coeficiente de transferencia calórica. Para resolver el sistema de ecuaciones diferenciales que representa la transferencia de energía en el sólido se debe implementar el método numérico. Esta técnica es la más adecuada si se asegura que la discretización espacial y temporal sea pequeña (Arce y Sweat, 1980).

Por lo tanto se procedió a construir un objeto acrílico de la misma geometría que el alimento bajo estudio. Para ello se utilizó un torno de control numérico, el cual actúa guiado por una computadora que ejecuta programas controlados por medio de datos alfa-numéricos, teniendo en cuenta los ejes cartesianos $x, y, z$. Se alimentó a la computadora que guiaba el torno el archivo CAD generado en Solidworks. Las propiedades termofísicas del acrílico en el rango estudiado son bien conocidas y pueden considerarse constantes dado que no presenta cambio de fase.

El prototipo de acrílico de la empanada se colocó en la cámara de refrigeración ubicando termocuplas en el centro geométrico, superficie del 
objeto y en el seno del fluido, con el objeto de conocer la historia térmica en dichos puntos. Luego se simularon las historias térmicas mediante el código computacional desarrollado y se compararon las salidas proponiendo distintos valores del coeficiente de transferencia de calor. Posteriormente se determinó el coeficiente de transferencia calórica (h) que representaba al sistema, según lo descripto en la Sección 3.3.2. De esta manera se obtuvo el coeficiente de transferencia calórica h promedio para el "objeto sumergido" en aire refrigerado considerando la geometría irregular del alimento.

5.2.7 Aplicación del modelo numérico para predecir tiempos de refrigeración para distintas condiciones de proceso

Una vez que el modelo numérico fue validado experimentalmente se lo utilizó para predecir tiempos de refrigeración variando distintos parámetros y condiciones del proceso, tales como el coeficiente de transferencia de calor y la temperatura del aire refrigerante.

\subsection{Resultados y discusión}

\subsubsection{Análisis de la malla utilizada para modelar la geometría irregular}

En la Figura 5-2 a) y b) se muestra una fotografía del producto real y la representación espacial generada en el software Solidworks del producto carneo-farináceo. En la Fig. 5-2 c) y d) se muestra una representación espacial del producto que permite la visualización de los elementos internos que corresponden a la fase cárnea y una visualización en perspectiva del dominio discretizado. 


\section{(F)}

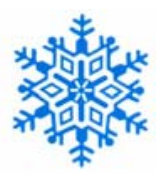

C I D C A

\subsubsection{Propiedades termofísicas de las fases (cárnea y farinácea) que componen el alimento}

El calor específico de la carne picada cocida y de la masa cruda en el rango de temperaturas estudiado, (entre $0^{\circ} \mathrm{C}$ y $25^{\circ} \mathrm{C}$ ), no presentaba variaciones significativas, por lo que se tomó un valor medio en el rango estudiado.
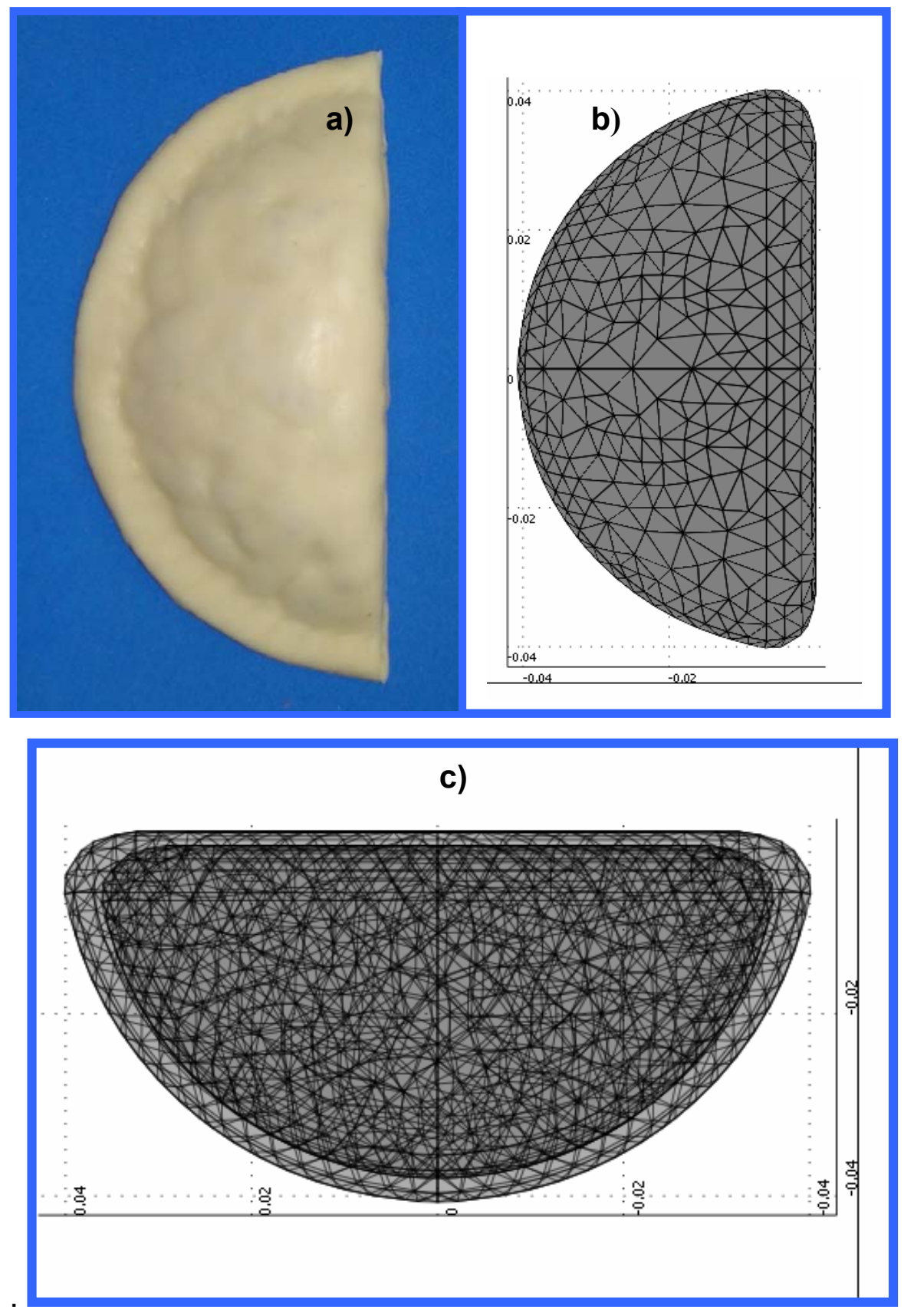


\section{(F)}

C I D C A

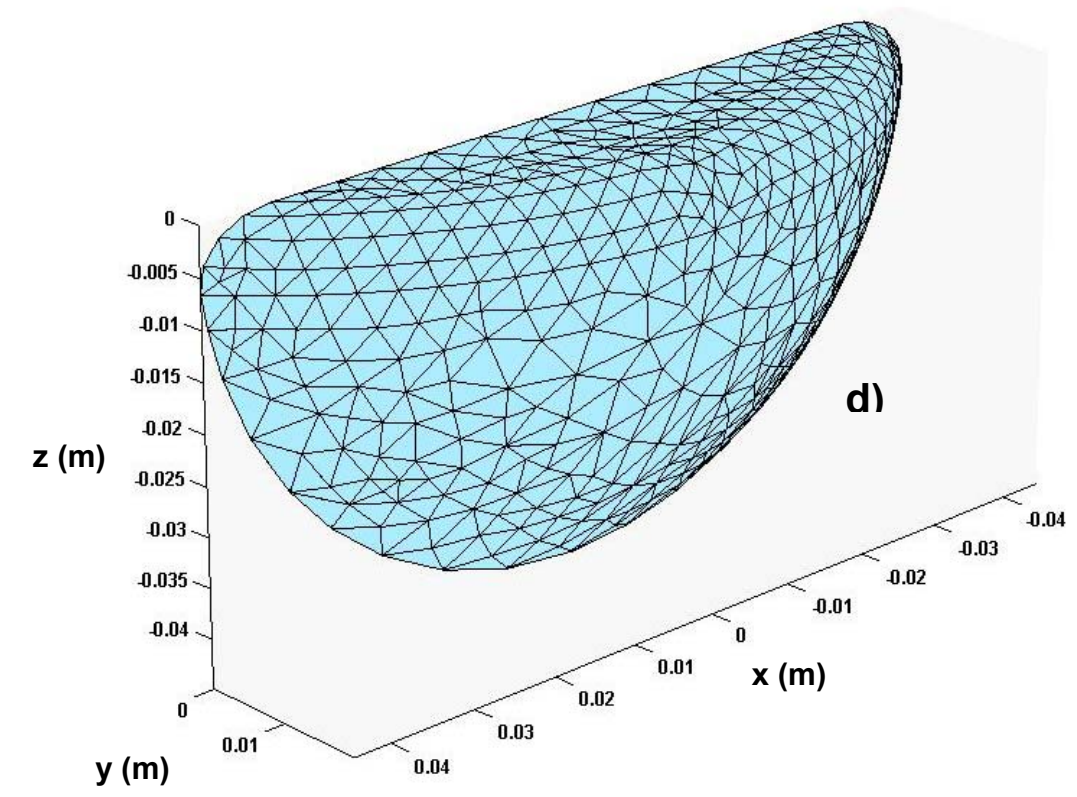

Fig. 5-2 a) Fotografía del producto (empanada). b) Discretización espacial tridimensional del producto de geometría irregular. c) Discretización del producto con visualización interna de cada fase del material, fase masa cruda ( $2 \mathrm{~mm}$ de espesor) y fase cárnica. d) Vista en perspectiva de la grilla representativa del producto

En la Fig. 5-3 se muestra a modo de ejemplo la curva de flujo de calor obtenida mediante DSC para ambos componentes en función de la temperatura (en el rango estudiado). Se puede observar que la masa presenta un pico endotérmico en el flujo de calor correspondiente a la fusión de la materia grasa que contiene.

El calor específico promedio (medido a través del DSC) de la fase cárnica cocida fue de $2951 \mathrm{~J} / \mathrm{kg}^{\circ} \mathrm{C}$ (en inglés "standard error of the mean" SEM = 20), y el de la masa $1938 \mathrm{~J} / \mathrm{kg}^{\circ} \mathrm{C}$ (SEM = 1). El contenido de agua de la fase cárnica fue del $66 \pm 3 \%$ y el de la masa de $30 \pm 2.5 \%$, según resultados obtenidos por deshidratación del alimento (ver Sección 5.2.3).

La masa utilizada tenía una composición de $47 \%$ carbohidratos, $30 \%$ de agua, $15 \%$ de lípidos, $6 \%$ de proteínas y $2 \%$ de fibra, valores informados por la planta elaboradora (La Salteña, General Mills Argentina). 
C I D C A

Curva de Flujo de Calor obtenida mediante DSC

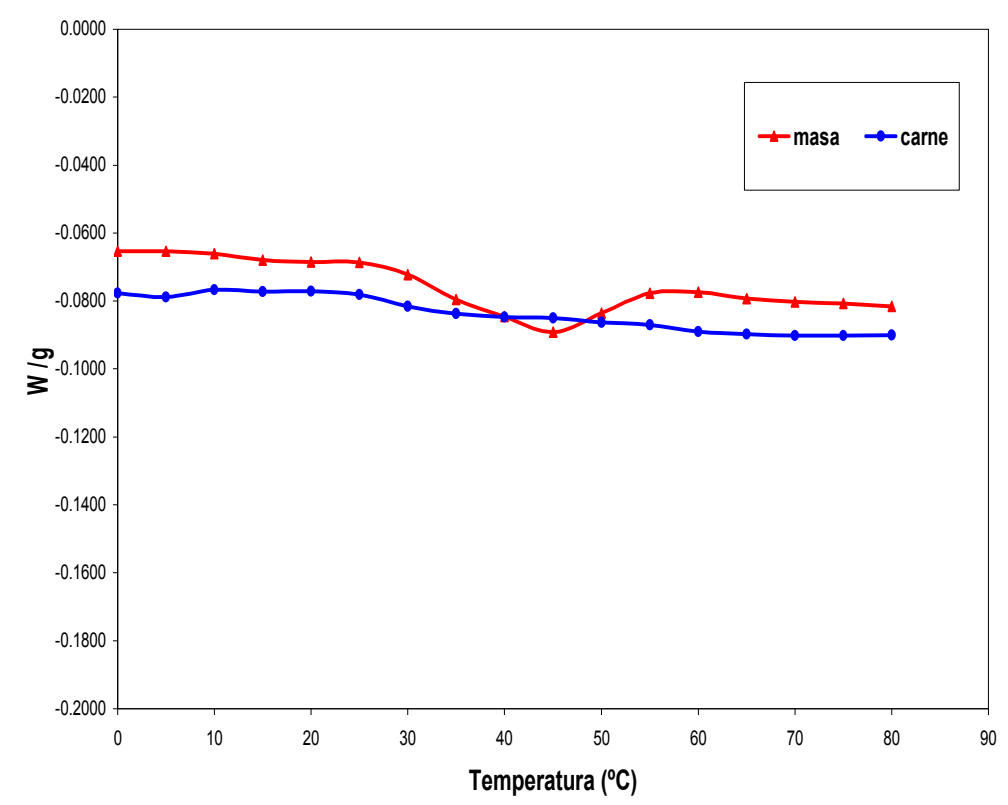

Fig. 5-3 Curva de flujo de calor (W/g) obtenida en DSC para determinar el calor específico para los dos componentes (masa cruda y carne magra picada cocida)

La dependencia de la conductividad térmica con la temperatura para la masa se estimó utilizando la siguiente ecuación (Choi y Okos, 1986).

$k(T)=\sum x_{i}^{v} \cdot k_{i}(T)$

donde: $\mathrm{k}$ es la conductividad global $\mathrm{y} \mathrm{k}_{\mathrm{i}}$ es la conductividad térmica del componente i donde i (agua, carbohidratos, cenizas, lípidos, proteínas, etc.); $\mathrm{x}_{\mathrm{i}}^{\mathrm{v}}$ corresponde a la fracción volumétrica de cada componente.

El valor de conductividad térmica de la masa no presentaba variaciones significativas con la temperatura por lo que se utilizó para las simulaciones el valor medio en el rango entre 0 y $25^{\circ} \mathrm{C}, \quad \mathrm{k}=0.3278 \mathrm{~W} / \mathrm{m}{ }^{\circ} \mathrm{C}(\mathrm{SEM}=0.0001$ $\mathrm{W} / \mathrm{m}^{\circ} \mathrm{C}$ ), lo cual concuerda satisfactoriamente con valores publicados de masa 
no leudada por Baik y col. (2001).

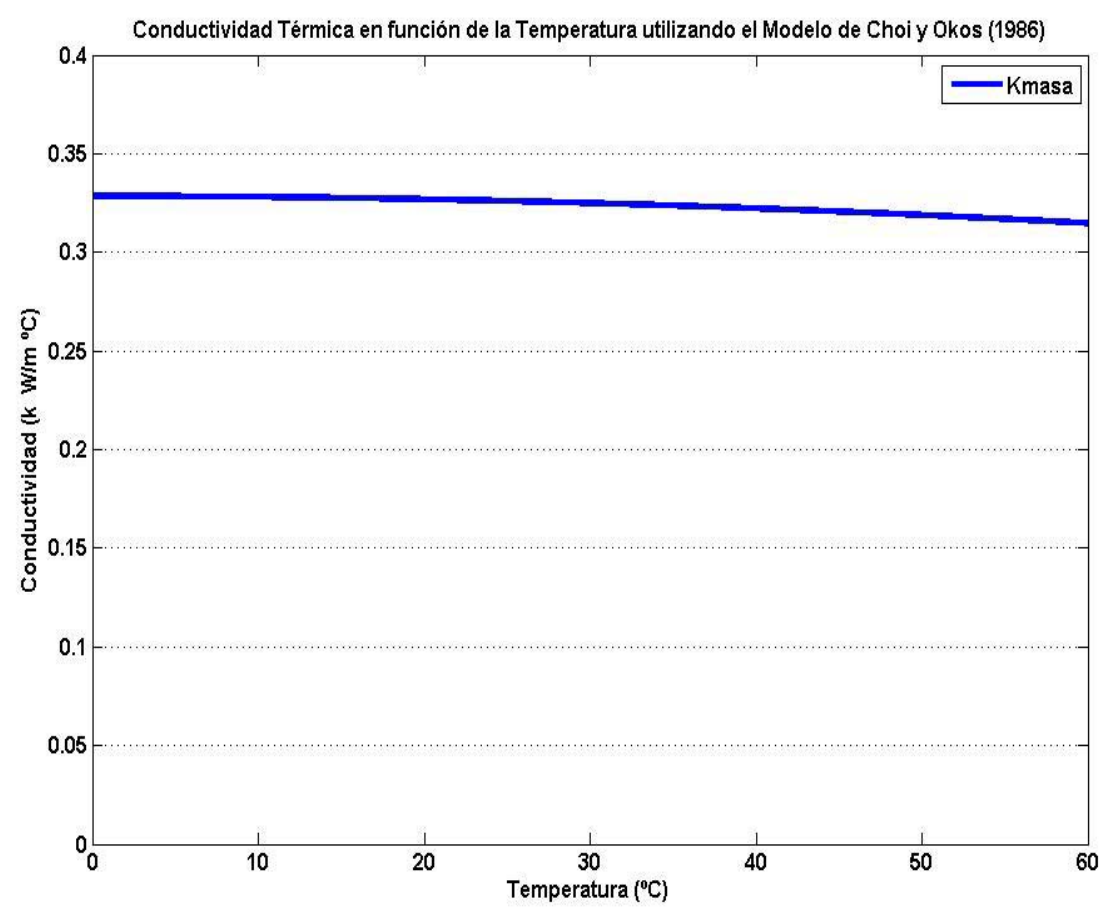

Fig. 5-4 Conductividad térmica de la masa en función de la temperatura utilizando el modelo de Choi y Okos (1986)

Para el cálculo de la conductividad térmica de la carne picada cocida se utilizó la siguiente ecuación (Sweat, 1975):

$\mathrm{k}=0.08+0.52 \cdot \mathrm{x}_{\mathrm{w}}$

donde $x_{w}$ corresponde al porcentaje de agua en el alimento. El valor de conductividad térmica de la carne picada cocida fue de $0.43 \mathrm{~W} / \mathrm{m}{ }^{\circ} \mathrm{C}$.

La densidad de la fase cárnica fue de $604 \mathrm{~kg} / \mathrm{m}^{3}$ y la de la masa cruda de $1221 \mathrm{~kg} / \mathrm{m}^{3}$. El peso promedio de las empanadas fue de $45.7 \pm 0.6 \mathrm{gr}$. 


\subsubsection{Coeficiente de transferencia calórica en interfase}

El coeficiente de transferencia calórica que minimizaba la varianza (ver Ec. 3.16) fue $\mathrm{h}=5.5 \mathrm{~W} / \mathrm{m}^{2}{ }^{\circ} \mathrm{C}$.

Además se utilizaron correlaciones de literatura (Earle, 1988; Nesvadba, 2008) para la estimación del coeficiente de transferencia en la interfase. Para un objeto sumergido en aire con convección natural el rango de coeficientes se encuentra entre 5 y $20 \mathrm{~W} / \mathrm{m}^{2}{ }^{\circ} \mathrm{C}$, lo cual concuerda con el valor obtenido experimentalmente. Los experimentos realizados en una cámara de refrigeración corresponden a este tipo de flujo ya que la velocidad de circulación de aire en la cámara era baja (se puede asumir como un enfriamiento en aire con convección natural.

\subsubsection{Validación experimental del modelo}

Los experimentos descriptos en la Sección 5.2.3 se utilizaron para validar el modelo numérico utilizando el MEF. En la Fig. 5-5 se puede observar las temperaturas predichas y las temperaturas experimentales en el punto, $(x, y, z)=(0,0.10,-0.01)$ del alimento, cuyas coordenadas están expresadas en metros, que corresponden al centro geométrico, para una de las experiencias realizadas. Además se muestra la temperatura externa media del aire $\left(5^{\circ} \mathrm{C}\right)$. Como puede observarse el modelo predice con buena exactitud el comportamiento de la temperatura en función del tiempo en el alimento.

Para todos los experimentos la norma infinito (valor máximo) del error absoluto fue menor a $1.3^{\circ} \mathrm{C}$ (error absoluto $=\mathrm{T}_{\text {exp }}-\mathrm{T}_{\text {pred }}$ ).

En la Figura 5-6 a) se puede observar la distribución de temperaturas en el producto luego de un tiempo de enfriamiento de 5 minutos. En la Figura 5-6 b) se muestra la distribución de temperaturas en un corte transversal ubicado en el plano de simetría $\mathrm{x}=0$ a un tiempo de residencia en la cámara de 5 minutos. En ambos casos la temperatura inicial del producto fue de $21.8^{\circ} \mathrm{C}$. 


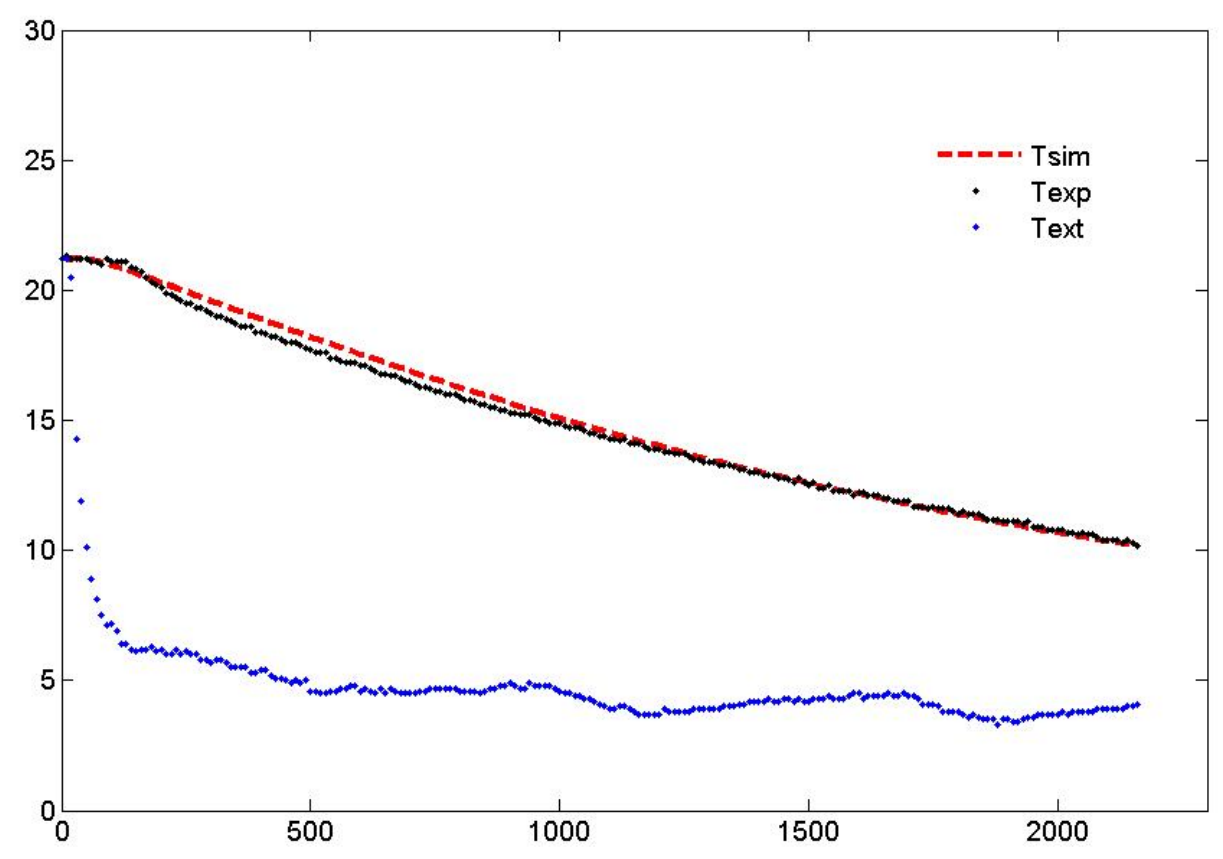

Fig. 5-5. Predicción numérica y datos experimentales de temperatura correspondiente al punto $(x, y, z)=(0,0.10,-0.01)$ coordenadas expresadas en $\mathrm{m}$, durante la refrigeración en una cámara a una temperatura promedio $\mathrm{T}_{\text {ext }}=5^{\circ} \mathrm{C}$. Temperatura inicial $21.8^{\circ} \mathrm{C}$, $\mathrm{h}=5.5 \mathrm{~W} / \mathrm{m}^{2}{ }^{\circ} \mathrm{C}$

\subsubsection{Aplicación del modelo en la predicción de tiempos de enfriamiento para distintas condiciones de proceso}

El modelo desarrollado y posteriormente validado, se utilizó para simular historias térmicas del proceso de refrigeración considerando una temperatura inicial uniforme de $20^{\circ} \mathrm{C}$ para distintas temperaturas del aire refrigerante en el equipo $\left(0,2,3^{\circ} \mathrm{C}\right)$, variando además el coeficiente de transferencia calórica. Se evaluó el tiempo necesario para alcanzar la temperatura final de $5^{\circ} \mathrm{C}$ en el punto más caliente del alimento. La Tabla 5-1 muestra los resultados de las simulaciones realizadas. En todos los casos el tiempo de cálculo computacional (tiempo $_{\text {срu }}$ ) fue menor a los 2.3 minutos utilizando una computadora Intel(R) Core(TM) 26300 con una velocidad de procesador de $1.86 \mathrm{GHz}$ y una memoria RAM de $2 \mathrm{~GB}$. 


\section{(F)}

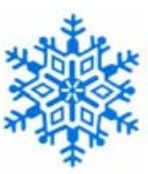

C I D C A
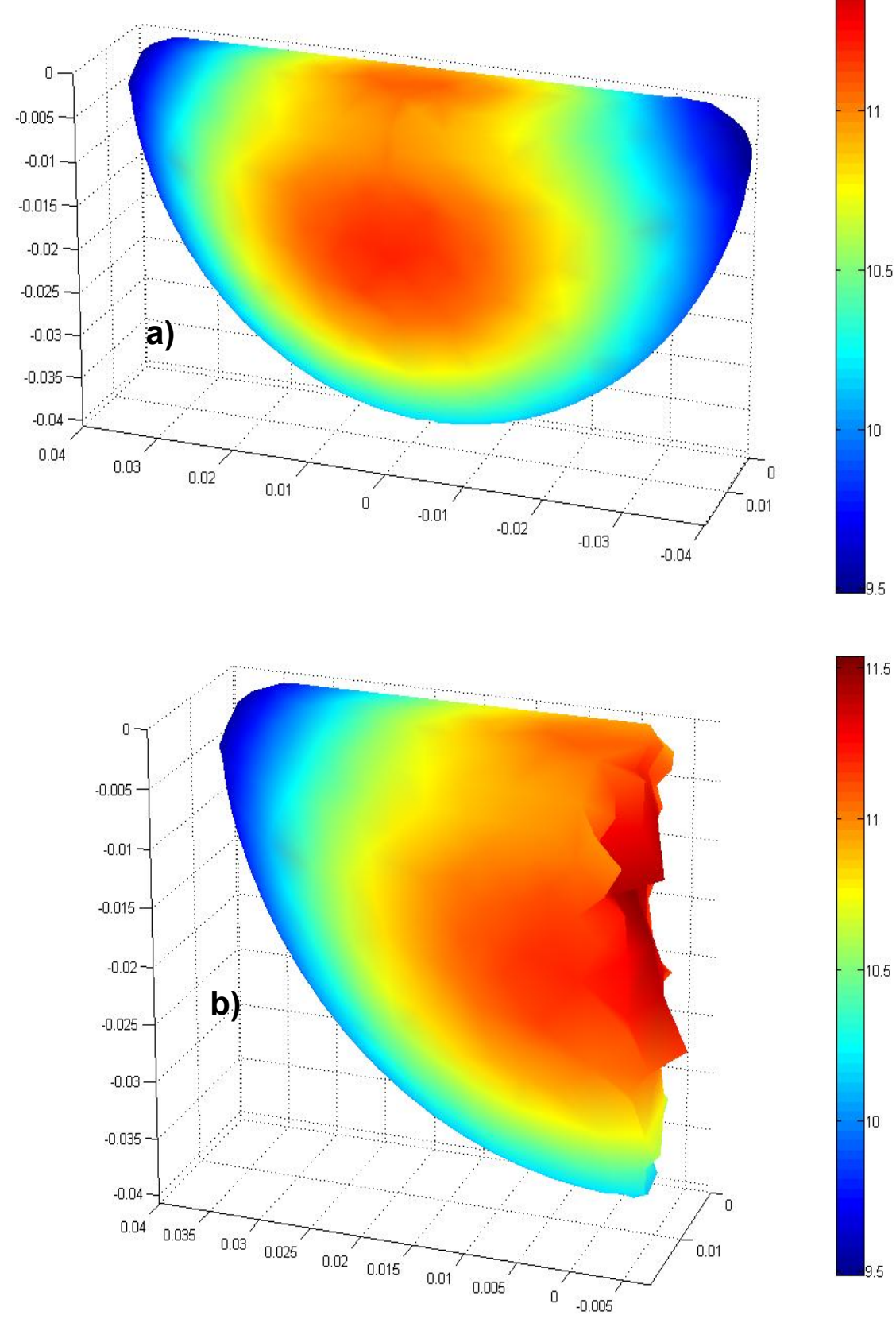

Fig. 5-6. Distribución de temperaturas en el producto luego de su refrigeración en una cámara a $5^{\circ} \mathrm{C}$ durante $5 \mathrm{~min}$. a) en la superficie b) en un corte transversal (Coeficiente de transferencia de calor, $h=5.5 \mathrm{~W} / \mathrm{m}^{2}{ }^{\circ} \mathrm{C}$, temperatura inicial, $\mathrm{Ti}=21.8^{\circ} \mathrm{C}$ ) 


\section{(5)}

C I D C A

Tabla 5-1. Tiempos de refrigeración ( $\mathrm{min}$ ) para distintos valores de $\mathrm{T}_{\text {ext }}$ y $\mathrm{h}$ (coeficiente de transferencia calórica)

\begin{tabular}{|cccccc|}
\hline \multicolumn{7}{c|}{ Tiempos de refrigeración $(\mathrm{min})$} \\
$\mathrm{h}\left(\mathrm{W} / \mathrm{m}^{2}{ }^{\circ} \mathrm{C}\right)$ & \\
\hline $\mathrm{T}_{\text {ext }}\left({ }^{\circ} \mathrm{C}\right)$ & 5 & 10 & 15 & 20 & 40 \\
\hline 0 & 46.6 & 25.6 & 18.5 & 14.8 & 9.1 \\
2 & 59.5 & 32.3 & 23.1 & 18.5 & 11.3 \\
3 & 70.3 & 38.0 & 27.1 & 21.6 & 13.2 \\
\hline
\end{tabular}

\subsection{Conclusiones}

Se desarrolló un modelo matemático que simula el proceso de refrigeración en un producto semi-elaborado (empanadas) de geometría irregular tridimensional y de composición heterogénea (fase cárnica y fase farinácea) utilizando el método de los elementos finitos.

Se determinaron experimentalmente el calor específico, la densidad y el contenido de agua de cada una de las regiones específicas que componen el alimento.

Se estimó mediante modelos y correlaciones la conductividad térmica y el coeficiente de transferencia de calor, los cuales fueron alimentados en la simulación numérica del problema.

El modelo fue validado experimentalmente obteniéndose una buena concordancia entre las historias térmicas predichas y las registradas en el laboratorio.

Se simularon historias térmicas para distintas condiciones de proceso (variando $T_{\text {ext }}$ y el coeficiente de transferencia calórico). El modelo presenta la versatilidad de poder conocer los tiempos mínimos de procesamiento de alimentos multifásicos de geometría irregular, alimentando las propiedades termofísicas correspondientes al programa computacional.

Es interesante resaltar que el programa desarrollado en lenguaje Matlab permite obtener resultados y predicciones precisas en tiempos mínimos de ejecución computacional, tiempo $\mathrm{cpu}_{\mathrm{c}}$ menor a los 3 minutos. En este aspecto el código numérico representa una herramienta útil para técnicos y profesionales que requieren información del proceso de manera eficiente y rápida. 
Capítulo 6 


\section{Simulación de problemas de transferencia de calor con cambio de fase en sistemas tridimensionales de geometría irregular utilizando el MEF}

\subsection{Introducción}

El proceso de congelación de alimentos representa un problema de conducción de calor con cambio de fase. En dichos problemas existen cambios abruptos en las propiedades térmicas de los alimentos, especialmente en el calor específico aparente, según lo explicado en el Capítulo 1 de la presente Tesis. Estas fuertes variaciones de las propiedades termofísicas con la temperatura generan que el planteo matemático del sistema sea altamente nolineal.

Existen limitaciones en los softwares comerciales de simulación por el MEF para resolver dichos problemas (Pham, 2008), ya que comúnmente utilizan un método en base al calor específico aparente. Si bien una forma de encarar la solución sería suavizando la función del $\mathrm{Cp}_{\mathrm{ap}}$ versus temperatura, esto alteraría tanto el problema matemático planteado originalmente como la realidad física, ya que el cambio real de las propiedades térmicas estaría representado por funciones "suavizadas", a veces muy distintas de las originales. Cabe señalar que en una primera etapa, se intentaron resolver problemas de transferencia de energía con cambio de fase (2D y 3D) aplicando un simulador comercial (COMSOL AB, 2005) en sistemas de geometría altamente irregular; sin embargo los resultados obtenidos no fueron satisfactorios ya que el programa comercial no funcionaba adecuadamente debido a que al utilizar una formulación basada en $\mathrm{Cp}_{\mathrm{ap}}$, se dificultaba la resolución de las matrices a cada paso del tiempo, conduciendo a la divergencia de la solución numérica. 


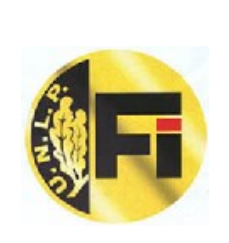

C I D C A

Por estas razones las formulaciones entálpicas se desarrollaron con éxito y fueron utilizadas por varios autores, donde la entalpía está definida como la integral del calor específico aparente con la temperatura (Comini, 1989; Pham, 2000). Por otro lado la ecuación de conducción de calor se puede reformular también en base a la conductividad térmica a través de la función de Kirchhoff $\left(E=\int k d T\right)$ donde la integral $E$ representa una función más suave comparado con la funcionalidad de la conductividad térmica versus $T$.

La combinación de ambos métodos de transformación de variables $\mathrm{Cp}$ aparente en entalpía y conductividad térmica según la función de Kirchhoff, resulta en una excelente formulación que elimina las inexactitudes causadas por la abrupta variación en las propiedades térmicas durante el cambio de fase. Esta metodología fue propuesta y utilizada por Fikiin $(1996,1998)$ y Scheerlinck y col. (2001). La combinación de estos métodos presenta diversas ventajas en comparación con formulaciones entálpicas o de Kirchhoff solamente.

Al implementar el método de los elementos finitos en la ecuación anterior los tiempos de ejecución computacional se ven ampliamente reducidos y esto permite resolver un gran número de problemas de cambio de fase, como optimización numérica o análisis de la propagación de incertidumbre utilizando el método de Monte Carlo en congelación de alimentos (Sheerlinck y col, 2001). Esta formulación además tiene la ventaja de poder resolver tanto problemas con cambio de fase gradual como abrupto, ya que utiliza las funciones integradas $\left(\mathrm{H}_{\mathrm{a}}\right.$ y $\left.\mathrm{E}\right)$ de las propiedades térmicas $\left(\rho, \mathrm{k}, \mathrm{C} \mathrm{p}_{\mathrm{ap}}\right)$. Para resolver un problema con cambio de fase utilizando las formulaciones entálpica y de Kirchhoff se requiere programar un código computacional propio en un lenguaje de programación.

Se seleccionó para la simulación la congelación de un alimento farináceo de geometría irregular (medialunas); este producto ocupa un lugar importante en la industria de panificados congelados. Además éstas resultan de gran interés por su amplia comercialización en la industria local.

El proceso de producción consiste básicamente en elaborar la masa 
cruda, la cual luego puede ser enfriada o congelada (con o sin el previo leudado) para su posterior transporte a los distintos establecimientos donde son horneadas según la demanda.

Los objetivos del presente Capítulo son:

> Desarrollar un código computacional propio que simule el proceso de congelación de un producto panificado de geometría irregular (croissantmedialunas) teniendo en cuenta la variación real de las propiedades termofísicas del alimento con la temperatura durante la congelación.

$>$ Medir experimentalmente el calor específico aparente del producto (que incluye el cambio de fase) utilizando Calorimetría Diferencial de Barrido (DSC).

$>$ Estimar el coeficiente de transferencia de calor en la interfase realizando experimentos de laboratorio en un túnel de congelación a escala piloto.

> Validar el modelo matemático mediante experimentos de congelación de los productos en un túnel escala piloto.

> Utilizar el modelo para predecir tiempos de congelación variando distintos parámetros y condiciones del proceso, como el coeficiente de transferencia de calor, la temperatura del aire, y temperatura inicial de producto.

\subsection{Materiales y métodos}

\subsubsection{Formulación matemática}

Los problemas no lineales en conducción de calor que involucran cambios de fase se pueden describir mediante la siguiente ecuación diferencial definida en el dominio $\Omega$ :

$\rho(T) \cdot C p(T) \frac{\partial T}{\partial t}=\nabla \cdot(k(T) \nabla T)$ 
donde $\mathrm{T}$ es la temperatura $\left({ }^{\circ} \mathrm{C}\right), \rho(\mathrm{T})$ la densidad $\left(\mathrm{kg} / \mathrm{m}^{3}\right), \mathrm{Cp}(\mathrm{T})$ el calor específico aparente que incluye el cambio de fase $\left(\mathrm{J} / \mathrm{kg}{ }^{\circ} \mathrm{C}\right), \mathrm{k}(\mathrm{T})$ es conductividad térmica $\left(\mathrm{W} / \mathrm{m}^{\circ} \mathrm{C}\right)$ y t representa el tiempo (s).

Se consideró que el cuerpo a congelar se encuentra inicialmente a una temperatura uniforme y es enfriado en un equipo de congelación operando a una temperatura constante, con un coeficiente de transferencia calórico en la interfase.

Las condiciones iniciales y de borde son las siguientes:

$$
\begin{array}{lll}
T=T_{0} & \text { en } \Omega & \text { a } t=0 \\
-k(T)(\nabla T \cdot n)=h\left(T-T_{\text {ext }}\right) & \text { en } \delta \Omega & \text { a } t>0
\end{array}
$$

donde $\mathrm{T}_{0}$ es la temperatura inicial $\left({ }^{\circ} \mathrm{C}\right), \mathrm{T}_{\text {ext }}$ es la temperatura externa del medio refrigerante $\left({ }^{\circ} \mathrm{C}\right)$, $n$ es el vector normal unitario, h el coeficiente de transferencia calórica $\left(\mathrm{W} / \mathrm{m}^{2}{ }^{\circ} \mathrm{C}\right)$, y $\delta \Omega$ la superficie del alimento expuesta a una condición de borde convectiva.

Se utilizó una transformación de variable a través de la entalpía volumétrica $\mathrm{H}_{\mathrm{a}}$ (Comini et al., 1990) definida como

$$
H_{a}(T)=\int_{T_{\text {ref }}}^{T} \rho(T) C p_{\text {ap }}(T) d T
$$

donde $\mathrm{T}_{\text {ref }}$ es una temperatura de referencia que corresponde a la entalpía cero. De la misma forma la conductividad térmica fue reemplazada utilizando la transformación de Kirchhoff (Comini y col., 1990: Saro y col., 1995; Fikiin, 1996): 
$E(T)=\int_{T_{\text {ref }}}^{T} k(T) d T$

A partir de las ecuaciones 6.4-6.5 se deben construir 6 relaciones entre las variables: $H_{a}(T), E(T), T\left(H_{a}\right), T(E), H(E), y E\left(H_{a}\right)$. Todas estas son monótonamente crecientes, continuas y biyectivas porque $\rho(T), C p(T), y k(T)$ son positivas y acotadas (Fikiin, 1996).

Sustituyendo las ecuaciones 6.4-6.5 en las ecuaciones 6.1-6.3 se obtiene el siguiente sistema de ecuaciones diferenciales parciales con dos variables dependientes e interrelacionadas $\mathrm{H}$ y $\mathrm{E}$ :

$\begin{array}{lccc}\frac{\partial \mathrm{H}_{\mathrm{a}}}{\partial \mathrm{t}}=\nabla^{2} \mathrm{E} & \text { en } \Omega & & 6.6 \\ \mathrm{H}_{\mathrm{a}}=\mathrm{H}_{\mathrm{a} 0} & \text { en } \Omega & \text { a } \mathrm{t}=0 & 6.7 \\ -(\nabla \mathrm{E} \cdot \mathrm{n})=\mathrm{h}\left(\mathrm{T}-\mathrm{T}_{\text {ext }}\right) & \text { en } \delta \Omega & \text { a } \mathrm{t}>0 & 6.8\end{array}$

donde $\mathrm{H}_{\mathrm{a} 0}=\mathrm{H}_{\mathrm{a}}\left(\mathrm{T}_{0}\right)$. De este modo las no linealidades de las propiedades dependientes de la temperatura en el alimento son eliminadas de las ecuaciones 6.1 a 6.3 e incorporadas como una función que vincula la entalpía volumétrica $\mathrm{H}_{\mathrm{a}}$ con la función de Kirchhoff.

\subsubsection{Formulación por el MEF}

Para la formulación por elementos finitos se aplicó el método de residuos ponderados de Galerkin (Zienkiewicz y Taylor, 1994a) y b)) obteniéndose el siguiente sistema: 


\section{(F)}

$C G \cdot \frac{d H_{a}}{d t}+K G \cdot E+M G \cdot T=F G$

donde

$$
\begin{array}{ll}
\mathrm{CG}=\sum_{\mathrm{e}=1}^{\mathrm{ne}} \int_{\Omega_{\mathrm{e}}}\left(\mathrm{H}^{\mathrm{t} H} \mathrm{H}\right) \mathrm{d} \Omega_{\mathrm{e}} & \mathrm{KG}=\sum_{\mathrm{e}=1}^{\mathrm{ne}} \int_{\Omega_{\mathrm{e}}}\left(\mathrm{B}^{\mathrm{t}} \mathrm{B}\right) \mathrm{d} \Omega_{\mathrm{e}} \\
\mathrm{MG}=\sum_{\mathrm{s}=1}^{\mathrm{ns}} \int_{\delta \Omega_{\mathrm{s}}}\left(\mathrm{H}^{\mathrm{t}} \mathrm{hH}\right) \mathrm{d} \delta \Omega_{\mathrm{s}} & \mathrm{FG}=\sum_{\mathrm{s}=1}^{\mathrm{ns}} \int_{\delta \Omega_{\mathrm{s}}}\left(\mathrm{H}^{\mathrm{t}} \mathrm{h} \mathrm{T}_{\text {ext }}\right) \mathrm{d} \delta \Omega_{\mathrm{s}}
\end{array}
$$

siendo $\mathrm{H}$ el vector de las funciones de forma, ne el número total de elementos de la malla y ns el número de elementos en contacto con la superficie expuesta a la condición de borde convectiva. Las matrices CG, KG y MG son las matrices globales de capacitancia, conductancia y matriz convectiva, respectivamente. El vector $F G$ es el vector de fuerzas globales que depende del coeficiente de transferencia de calor y de la temperatura externa. $\mathrm{H}_{a}$, E y $T$ son los vectores nodales de entalpía volumétrica, de la función Kirchhoff, y de la temperatura respectivamente.

Se puede observar que gracias a la simultánea transformación de variables en entalpía y en la función de Kirchhoff todas las no-linealidades y dependencias de temperatura de las propiedades fueron eliminadas y como consecuencia las matrices en la formulación por elementos permanecen constantes (no aparecen $\mathrm{k}, \mathrm{Cp}$ y $\rho$ en el cálculo de matrices y vectores elementales); esto significa que las matrices se calculan por fuera del ciclo que avanza en el tiempo reduciendo significativamente el tiempo de máquina (Sheerlinck y col., 2001). En otras palabras, el método permitió transformar un un problema altamente no-lineal en uno lineal gracias a la incorporación de las variables $E$ y $H_{a}$. Existen muchos trabajos donde se aplica la variable entalpía en procesos de congelación, sin embargo no es frecuente encontrar trabajos con una simultánea transformación entálpica y de Kirchhoff, siendo este un 
aporte muy interesante en lo referente a la simulación de problemas no-lineales de congelación.

Reemplazando las funcionalidades de $\mathrm{E}\left(\mathrm{H}_{\mathrm{a}}\right)$ y $\mathrm{T}\left(\mathrm{H}_{\mathrm{a}}\right)$ en la ecuación 6.9 resulta el siguiente sistema de ecuaciones diferenciales ordinarias.

$\frac{d H_{a}}{d t}=f\left(H_{a}, t\right)$

La solución del sistema de ecuaciones diferenciales ordinarias necesaria para avanzar en el tiempo se implementó mediante un método implícito con un $\Delta$ t variable (Subrutina ODE).

El código computacional aplicando la formulación de Kirchhoff y de Entalpía se programó en Matlab 6.5. Se implementaron las mismas optimizaciones del código computacional que las descriptas en el Capítulo 5.

\subsubsection{Sistema experimental}

El producto farináceo de geometría irregular (medialunas) se elaboró cortando triángulos isósceles de masa cruda comercial de $15 \mathrm{~cm}$ de base y 21 $\mathrm{cm}$ de altura, que luego fueron arrolladas en forma pareja desde la base hacia el vértice superior. Se congelaron muestras de productos en un túnel de congelación en escala piloto, el cual permite obtener condiciones operativas similares a las de la industria, registrando la temperatura en función del tiempo en varios puntos del alimento y en el medio externo por medio de termocuplas conectadas a un adquisidor de datos (Testo 175, Testo AG, Alemania). La velocidad del aire refrigerante en el túnel se midió mediante un anemómetro de hilo caliente (TSI modelo 1650).

\subsubsection{Generación de la malla}

La representación espacial tridimensional del objeto de geometría 
irregular se obtuvo a través de archivos CAD generados mediante el software Solidworks. Estos archivos fueron luego ingresados al mallador para su discretización en elementos tetraédricos. Para esto se prepararon varias muestras las cuales se dibujaron tomando las medidas espaciales con un calibre.

\subsubsection{Determinación experimental del calor específico y del coeficiente de transferencia de calor en la superficie.}

El cálculo del calor específico $\mathrm{Cp}_{\mathrm{ap}}$ se realizó siguiendo el mismo procedimiento descripto en la Sección 5.2.5 del Capítulo anterior utilizando un Calorímetro Diferencial de Barrido (DSC).

Para estimar el coeficiente de transferencia de calor se procedió de la misma forma que la descripta en la Sección 5.2.6. Se construyó un objeto de acrílico del cual se conocen las propiedades térmicas cuya geometría era igual a la del producto a congelar. El prototipo de acrílico se colocó en el túnel de congelación ubicando termocuplas en el centro geométrico, superficie del objeto y en el seno del fluido, con el objeto de conocer la historia térmica en dichos puntos. Luego se simularon las historias térmicas del centro geométrico mediante el código computacional desarrollado y se compararon las salidas proponiendo distintos valores del coeficiente de transferencia de calor.

\subsubsection{Aplicación del modelo numérico para predecir tiempos de congelación bajo distintas condiciones de proceso}

Se utilizó el modelo para predecir tiempos de congelación variando distintos parámetros y condiciones del proceso, como el coeficiente de transferencia de calor, la temperatura del aire, y temperatura inicial de producto. Posteriormente se obtuvo la superficie de respuesta que representa los tiempos de congelación (min) en función de la temperatura y el coeficiente de transferencia de calor en la interfase $(h)$. 


\subsection{Resultados y discusión}

\subsubsection{Análisis de la malla utilizada para modelar la geometría irregular}

En la Figura 6-1 a) y b) se muestra una imagen real y la malla generada a partir de la geometría irregular del producto panificado. Previo al mallado se realizó la representación espacial en el software Solidworks. Como puede apreciarse las medialunas presentan pliegues característicos debido a la forma en que son elaboradas, sin embargo estos pliegues son en todos los casos menores a 1-1.5 mm de espesor. Estos bordes generados en los archivos CAD traen dificultades cuando se genera la malla de los mismos en la superficie del alimento, debido a que los triángulos y tetraedros requeridos deben ser muy pequeños para que permitan aproximar la geometría real con exactitud, aumentándose así el número de tetraedros de la malla final. En consecuencia se aumentan los esfuerzos computacionales sin obtener ventajas apreciables en la solución numérica e incrementando el tiempo de cálculo de manera innecesaria. Por esta razón se generó otro archivo representativo del producto en el cual se interpolan las superficies entre los pliegues generando una única superficie suave, la cual genera una cantidad aceptable de elementos tetraédricos y triangulares para su posterior procesamiento. La Figura 6-1 c) muestra el producto con su geometría simplificada.

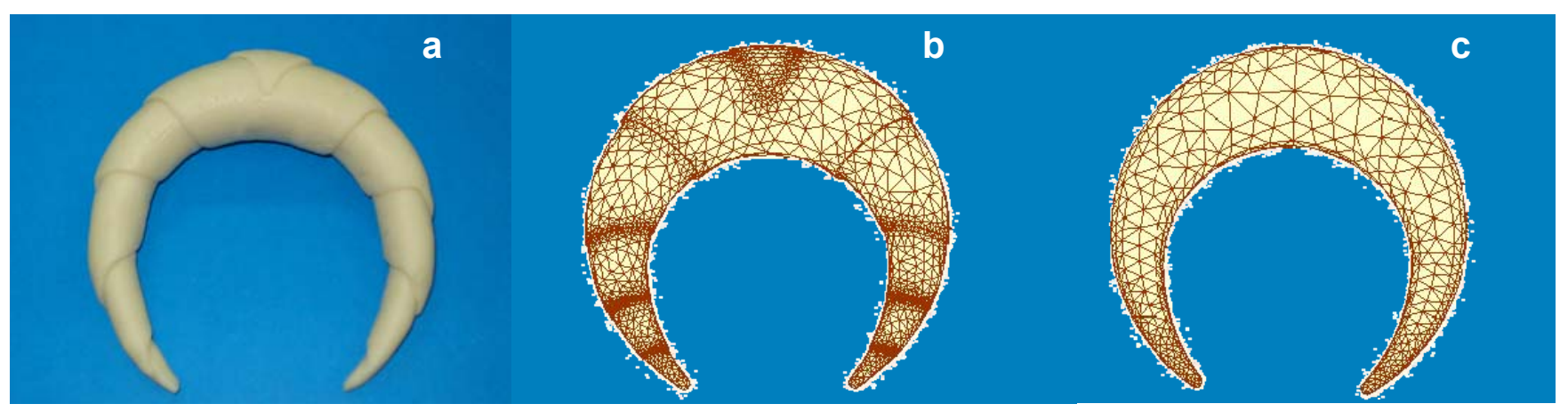

Fig. 6-1. a) Imagen del producto de geometría irregular; b) Mallado Completo (25000 elementos y 6000 nodos aproximadamente); c) Mallado Simplificado (5000 elementos y 1400 nodos aproximadamente) 


\section{(F)}

C I D C A

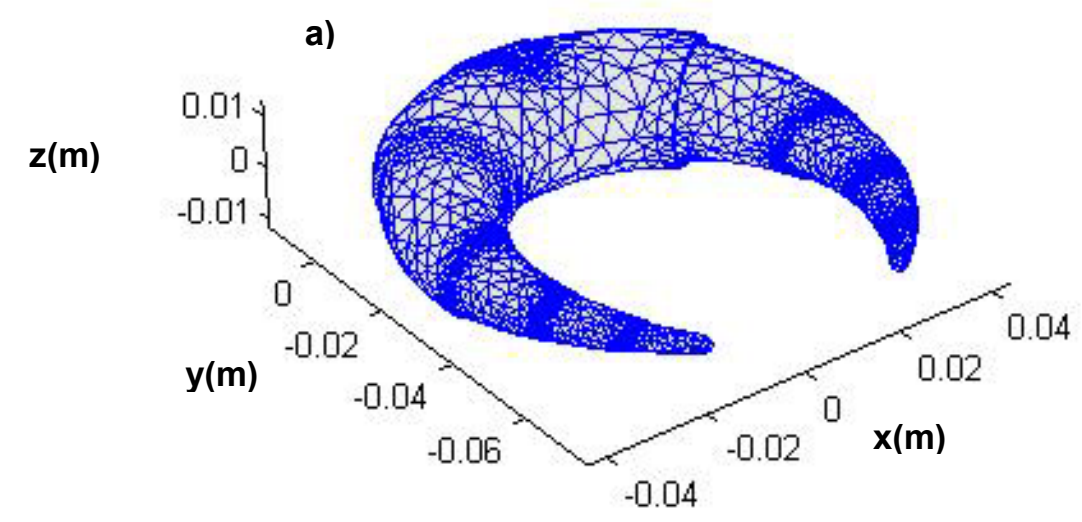

b)

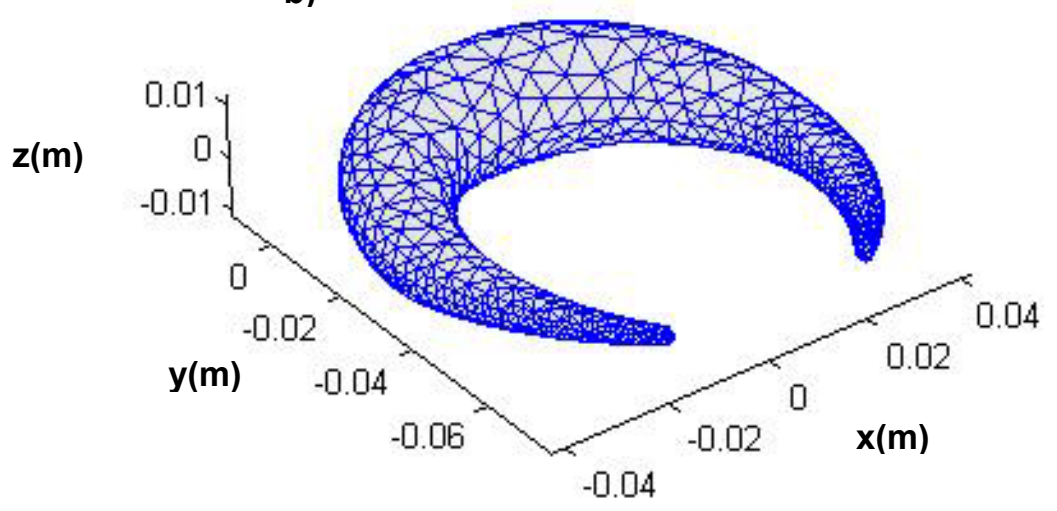

Fig. 6-2 a) Vista en persectiva del mallado completo de la imagen del producto panificado (25000 elementos y 6000 nodos aproximadamente); b) Vista en persectiva del mallado Simplificado (5000 elementos y 1400 nodos aproximadamente)

\subsubsection{Propiedades termofísicas}

La masa utilizada tenía una composición centesimal de $47 \%$ carbohidratos, $30 \%$ de agua, $15 \%$ de lípidos, $6 \%$ de proteínas y $2 \%$ de fibra, dada por la planta elaboradora (La Salteña, General Mills, Argentina).

La dependencia de la conductividad térmica con la temperatura se estimó utilizando un modelo en paralelo (Choi y Okos, 1986).(Fig.6-3)

$$
k(T)=\sum x_{i}^{v} \cdot k_{i}(T)
$$


donde $\mathrm{k}$ es la conductividad global $\mathrm{y}_{\mathrm{i}}$ es la conductividad térmica del componente i donde i ( agua, carbohidratos, cenizas, lípidos, proteínas, y hielo si la temperatura se encuentra por debajo de la temperatura inicial de fusión).

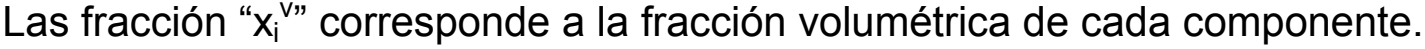

La cantidad de hielo que se forma se estimó según Miles (1974) como:

$x_{h}=\left(x_{w}-x b\right)\left(1-\frac{T f}{T}\right)$

donde Tf es la temperatura de inicio de fusión del producto, $x_{h}$ es la fracción másica de hielo, $x_{w}$ es la fracción másica de agua en el alimento, y xb es la fracción másica de agua ligada no congelada. La fracción de agua no congelada se estimó experimentalmente a partir del termograma de descongelación utilizando el DSC. El cociente entre el calor de fusión del material, el cual se obtuvo integrando el pico endotérmico del termograma, y el calor de fusión del agua pura, $333.2 \mathrm{~J} / \mathrm{g}$ (Weast y Astle, 1981) representa la fracción de agua congelada en el alimento. El xb se calculó como la diferencia entre el contenido de agua total del alimento y el contenido de agua congelable (Sá y col., 1994). El xb para la masa utilizada fue de $18.4 \%$ lo cual concuerda con datos de literatura para productos panificados crudos no leudados (“dough") (Lind, 1991). 


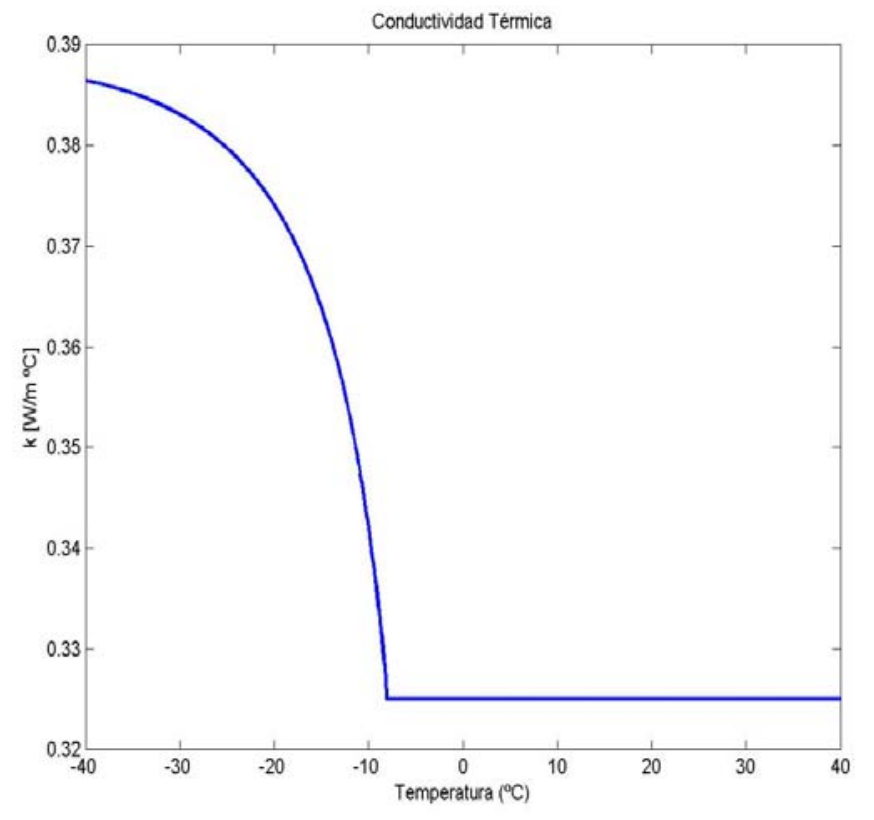

Fig. 6-3. Conductividad térmica del producto en función de la temperatura

La temperatura inicial de congelación $\mathrm{Tf}$, se determinó experimentalmente a partir curvas de congelación utilizando el método de la tangente descripto en Fennema y col. (1973). La temperatura inicial de congelación de la masa utilizada para formular las medialunas fue de $-7.5^{\circ} \mathrm{C}$.

La densidad del producto se obtuvo utilizando el modelo propuesto por Choi y Okos (1983):

$$
\rho(T)=\frac{1}{\sum \frac{x_{i}}{\rho_{i}}}
$$

donde $\rho(T)$ es la densidad global y $\rho_{i}$ es la densidad del componente i donde i (agua, carbohidratos, cenizas, lípidos, proteínas, y hielo si la temperatura se encuentra por debajo de la temperatura inicial de fusión). Las fracción " $x_{i}$ " corresponde a la fracción másica de cada componente. En el siguiente gráfico 
se puede observar la funcionalidad de la densidad con la temperatura (Fig. 6$4)$.

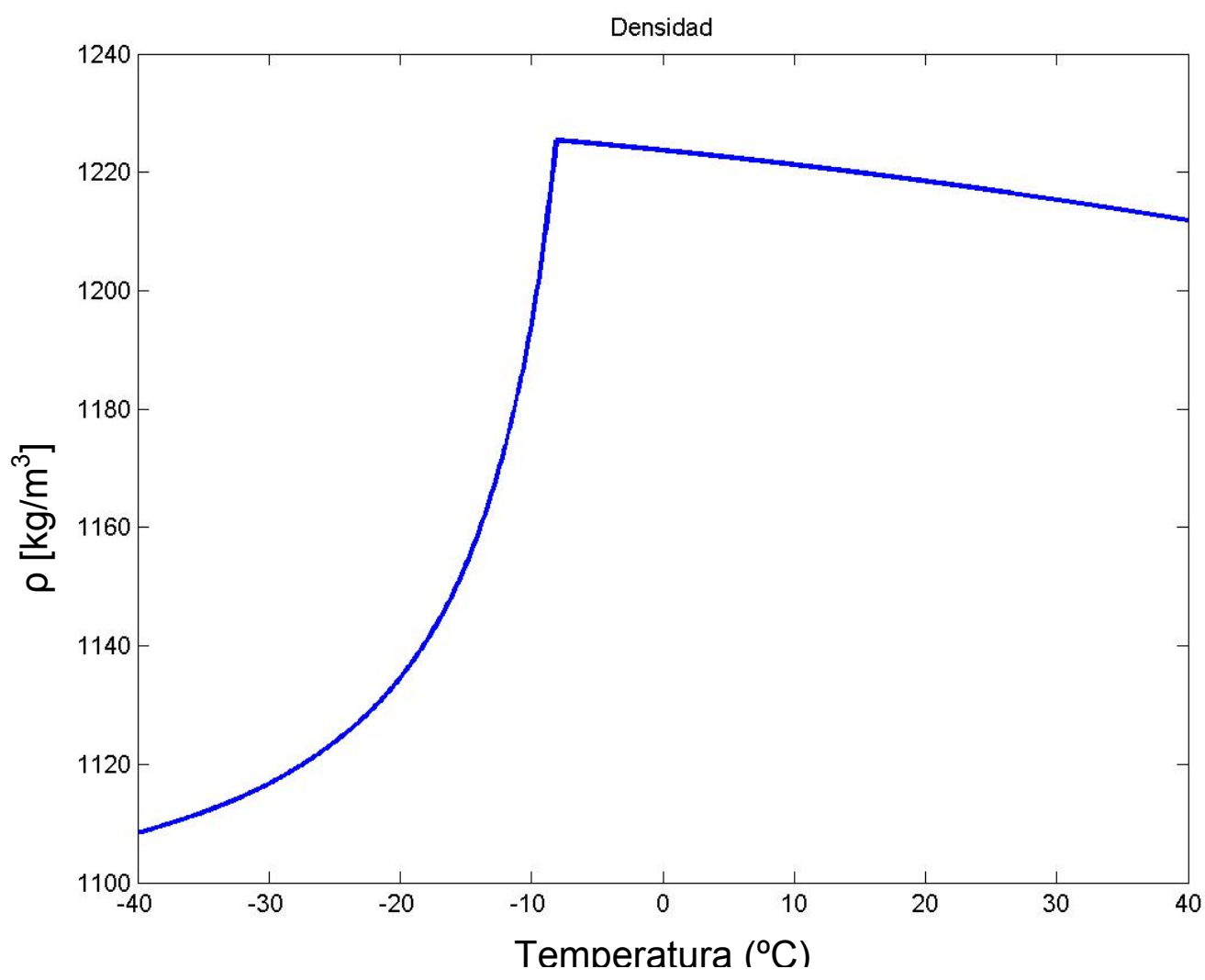

Fig. 6-4. Densidad del producto en función de la temperatura

En la Figura 6-5 se muestra la variación del $\mathrm{Cp}_{\mathrm{ap}}$ con la temperatura obtenida a partir de los experimentos realizados en DSC. El pico más notorio es debido a la congelación del agua del producto.

Se obtuvo el calor específico volumétrico multiplicando la densidad $\rho(T)$ por el $\mathrm{Cp}(\mathrm{T})$.

El cálculo de la entalpía volumétrica $\left(\mathrm{H}_{\mathrm{a}}\right)$ se realizó integrando numéricamente mediante el calor específico volumétrico tomando $-40^{\circ} \mathrm{C}$ como temperatura de referencia (Fig. 6-6 a) ). De la misma forma se procedió con la conductividad térmica obteniendo la función de Kirchhoff (E) (ver Fig. 6-6 b) y con las funciones $E\left(H_{a}\right)$ y $T\left(H_{a}\right)$ necesarias para obtener la Ec. 6.10 . (Fig. 6-7 a) y b)). 
C I D C A

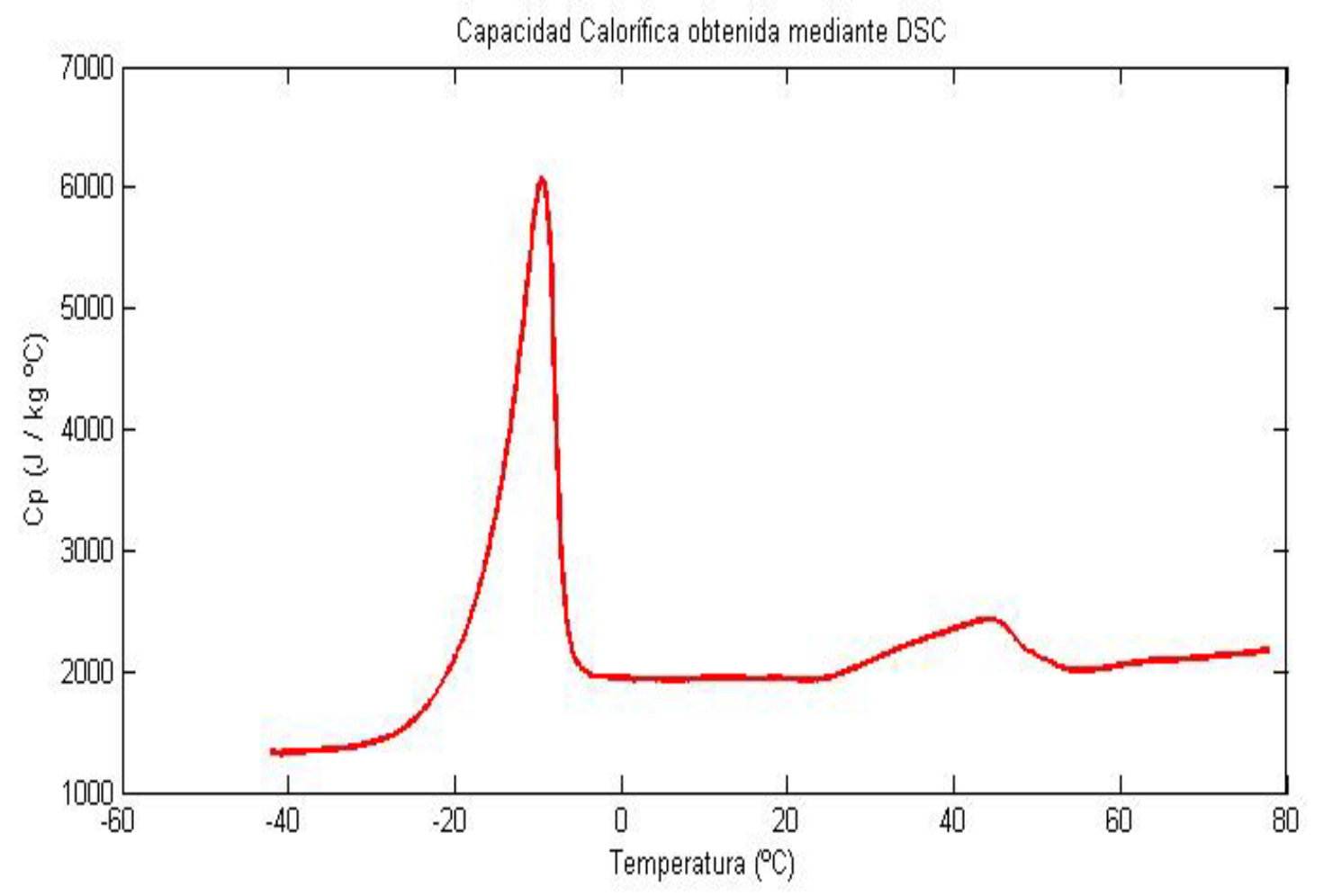

Fig. 6-5. Calor específico aparente en función de la temperatura obtenido a través de DSC
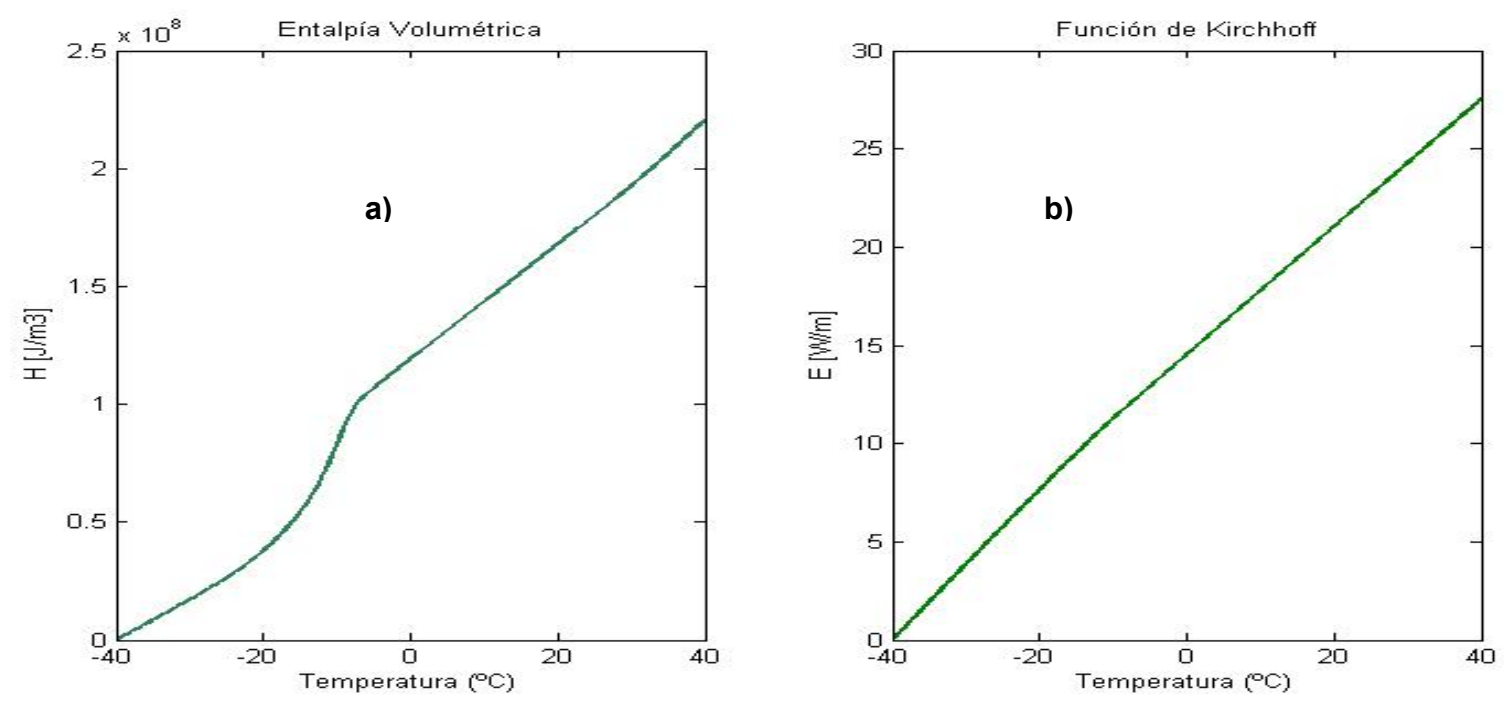

Fig. 6-6 a) Entalpía volumétrica; b) Función de Kirchhoff ambas en función de la temperatura. 
C I D C A

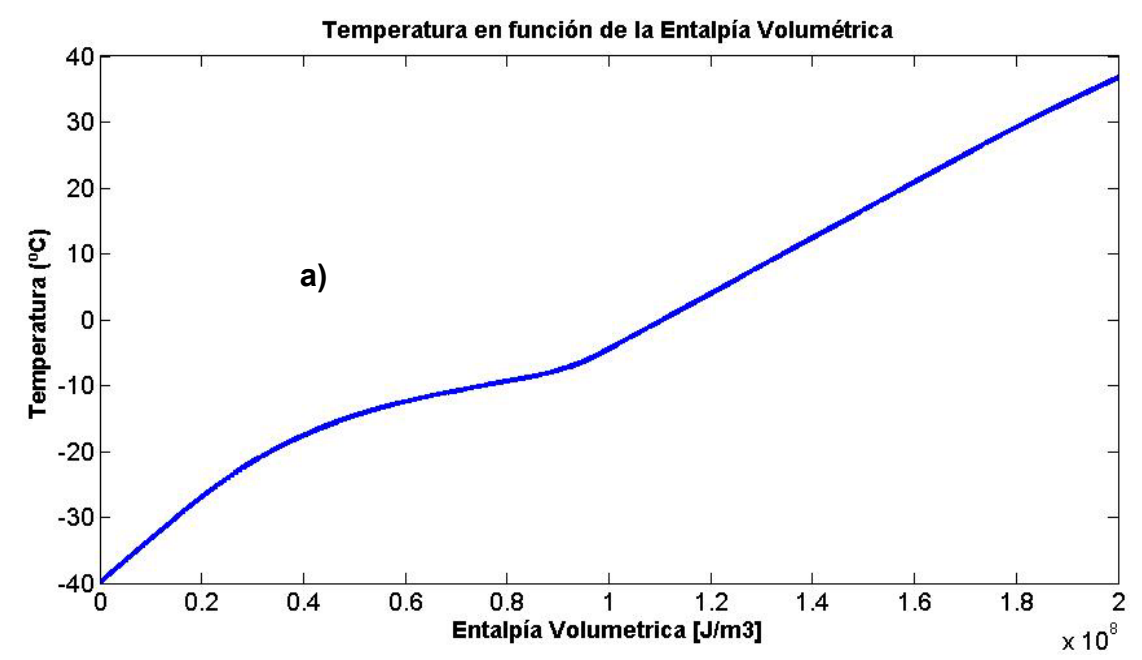

Fig. 6-7 a)Temperatura en función de la entalpía volumétrica

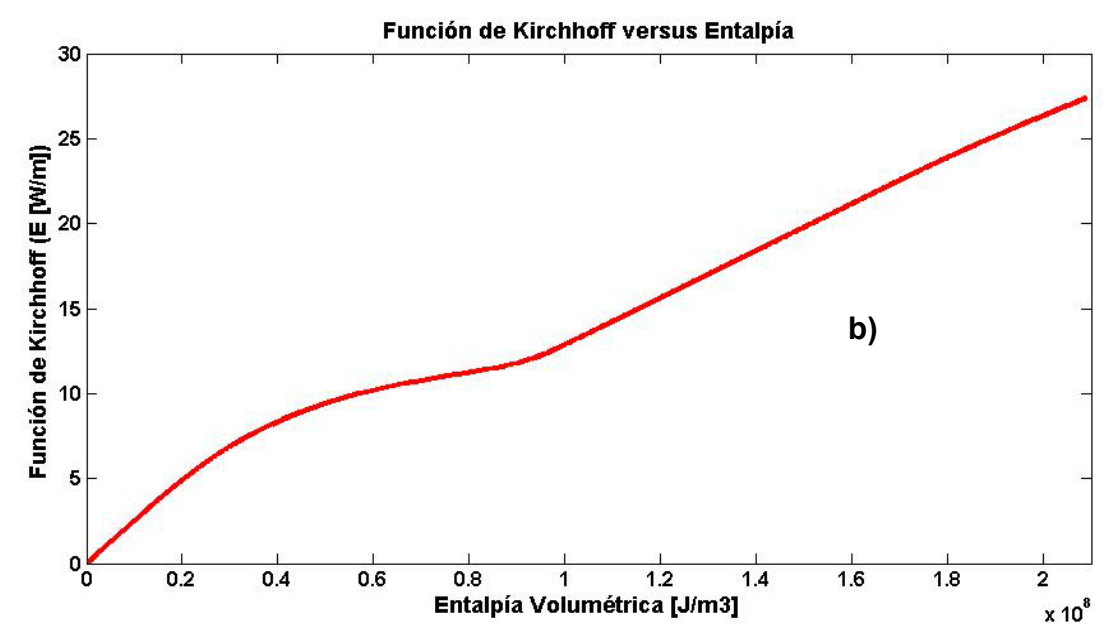

Fig. 6-7 b) Función de Kirchhoff en función de la Entalpía volumétrica

\subsubsection{Coeficiente de transferencia calórica (h)}

De acuerdo al método descripto en 6.2 .5 y las ecuaciones presentadas en el Capítulo 3, el coeficiente de transferencia calórica que mejor ajustaba los datos experimentales fue $\mathrm{h}=18 \mathrm{~W} / \mathrm{m}^{2}{ }^{\circ} \mathrm{C}$.

Además se utilizaron correlaciones de literatura donde obtenemos el número de $\mathrm{Nu}=\mathrm{hL} / \mathrm{k}$, en función del $\mathrm{Re}=\rho \mathrm{vL} / \mu$ y $\operatorname{Pr}=\mathrm{Cp} \mu / \mathrm{k}$ para estimar el $\mathrm{h}$ 
(Earle, 1988). En el caso de enfriamientos sobre superficies planas para convección forzada es recomendable la siguiente ecuación:

$\mathrm{Nu}=0.036 \cdot(\operatorname{Re})^{0.8}(\operatorname{Pr})^{0.33}$ para $\operatorname{Re}>2 \times 10^{4}$

Para calcular la longitud característica utilizada en el Re se adoptó el promedio entre la longitud máxima en la direcciones $x$, y de la geometría irregular de la medialuna, $\mathrm{L}=8.75 \mathrm{~cm}$. El valor de $\mathrm{h}$ obtenido mediante la Ec. 6.14 fue de $20.80 \mathrm{~W} / \mathrm{m}^{2}{ }^{\circ} \mathrm{C}$.

Si se trata específicamente de flujo de aire (para velocidades de aire $v<5 \mathrm{~m} / \mathrm{s}$ ) una de las ecuaciones que puede aplicarse para calcular $\mathrm{h}$ es (Earle, 1988):

$h=5.7+3.9 \cdot v$

De acuerdo con esta ecuación el valor obtenido fue de $15.5 \mathrm{~W} / \mathrm{m}^{2}{ }^{\circ} \mathrm{C}$. Por lo tanto se puede concluir que el valor estimado de $\mathrm{h}$ obtenido mediante el método transiente $\left(18 \mathrm{~W} / \mathrm{m}^{2}{ }^{\circ} \mathrm{C}\right)$ predice valores de coeficientes de tranferencia de calórica dentro del rango de valores estimados mediante correlaciones de literatura para convección forzada en objetos sumergidos.

\subsubsection{Validación experimental del modelo}

En la Figura 6-8 se puede observar una buena concordancia entre los resultados predichos por el programa de simulación codificado en Matlab 6.5 y las temperaturas experimentales obtenidas durante el proceso de congelación en el túnel. La experiencia mostrada a modo de ejemplo, correspondió a una temperatura de túnel de $-20{ }^{\circ} \mathrm{C}$, siendo el coeficiente de transferencia calórica de $18 \mathrm{~W} / \mathrm{m}^{2} \mathrm{~K}$ con una velocidad del aire de $2.5 \mathrm{~m} / \mathrm{s}$. El error absoluto promedio entre las temperaturas experimentales y predichas para todas las experiencias 
fue menor a los $2{ }^{\circ} \mathrm{C}$. En la Figura 6-9 a) y b) se muestra la distribución de temperaturas del producto sobre la superficie externa y en un corte transversal para visualizar las temperaturas internas en la sección de mayor espesor del alimento, a un tiempo de congelación de 25 minutos. Como se puede apreciar en los extremos del producto la temperatura es más baja dado que en esas zonas se registraron las velocidades de congelación más altas.

Es importante resaltar que la solución numérica es estable aun en los extremos agudos del dominio irregular, lo cual muestra la bondad de la formulación.

\subsubsection{Cálculo de tiempos de congelación para distintas condiciones de proceso}

El modelo desarrollado se utilizó para simular historias térmicas del proceso de congelación considerando una temperatura inicial de $10^{\circ} \mathrm{C}$ para distintas temperaturas del aire refrigerante en el equipo variando además el coeficiente de transferencia calórica. Se evaluó el tiempo necesario para alcanzar la temperatura final de congelación de $-18^{\circ} \mathrm{C}$ en el punto más caliente del alimento. La Tabla 6-1 muestra los resultados de las simulaciones realizadas. En todos los casos el tiempo de cálculo (cpu time) fue menor a los 3 minutos cuando se utilizó la función ODE en una máquina X64 Intel(R) Core (TM) 2CPU con $1.86 \mathrm{GHz}$ y 2 GB de RAM. Como puede apreciarse el tiempo de ejecución del programa es comparable con el de simulación de un proceso de transferencia de calor sin cambio de fase (donde el tiempo de ejecución fue de 2.3 minutos). Esta ventaja del código propio se debe a la implementación de la formulación entálpica y de Kirchhoff, la cual transforma al problema no-lineal original en un problema más simple (lineal), dejando a las matrices y vectores constantes utilizando el MEF, debiendo calcularlas sólo una vez. 

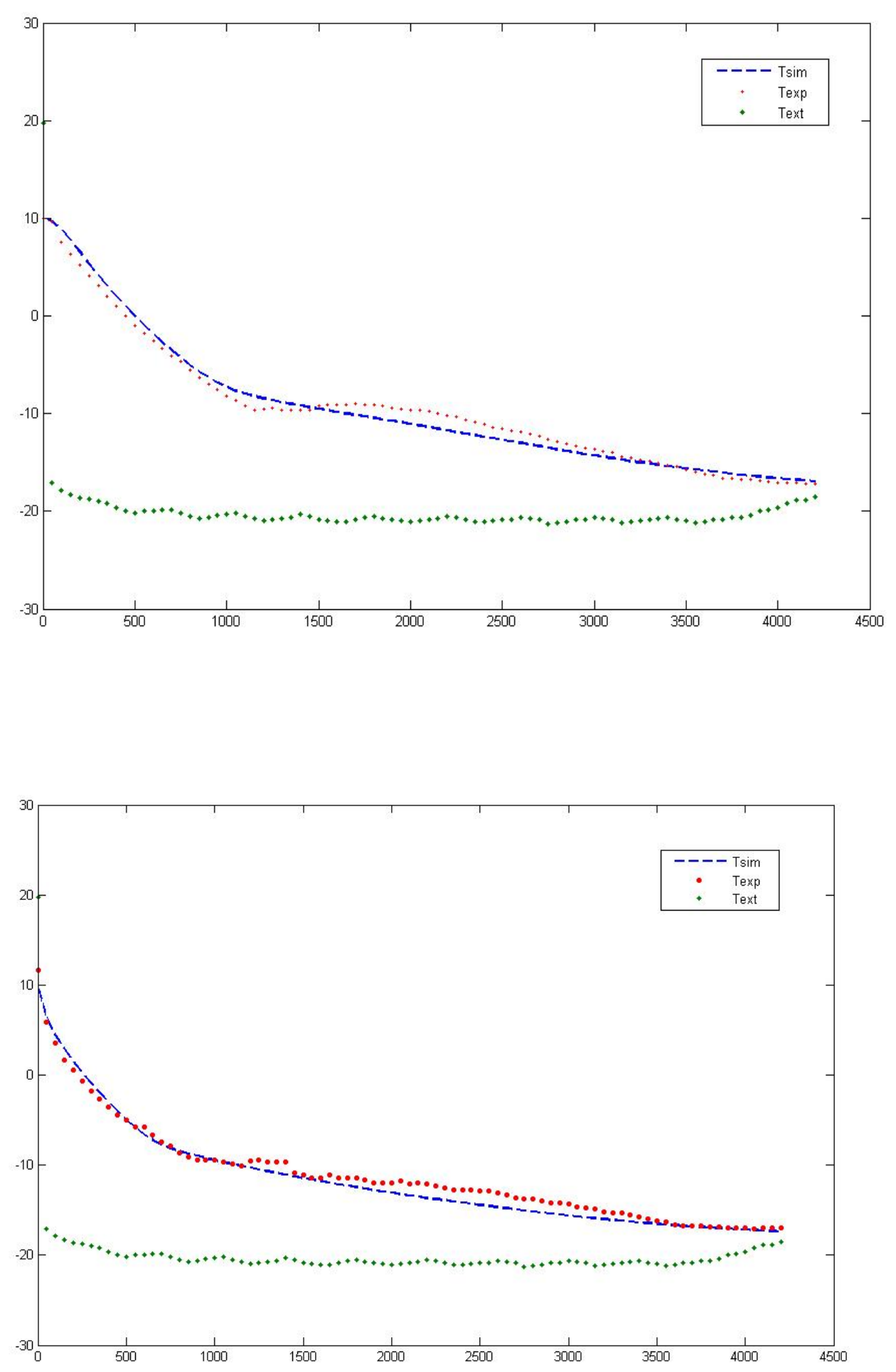

Fig. 6-8 Comparación entre las temperaturas experimentales y predichas durante la congelación en túnel para a) centro de la medialuna (en el punto $(0,0,0)$ y b) borde (punto $(0.020,-0.005,0.010)$ ). $\mathrm{T}_{\mathrm{ext}}=-20^{\circ} \mathrm{C}$ y $\mathrm{T}_{0}=10^{\circ} \mathrm{C}$ 

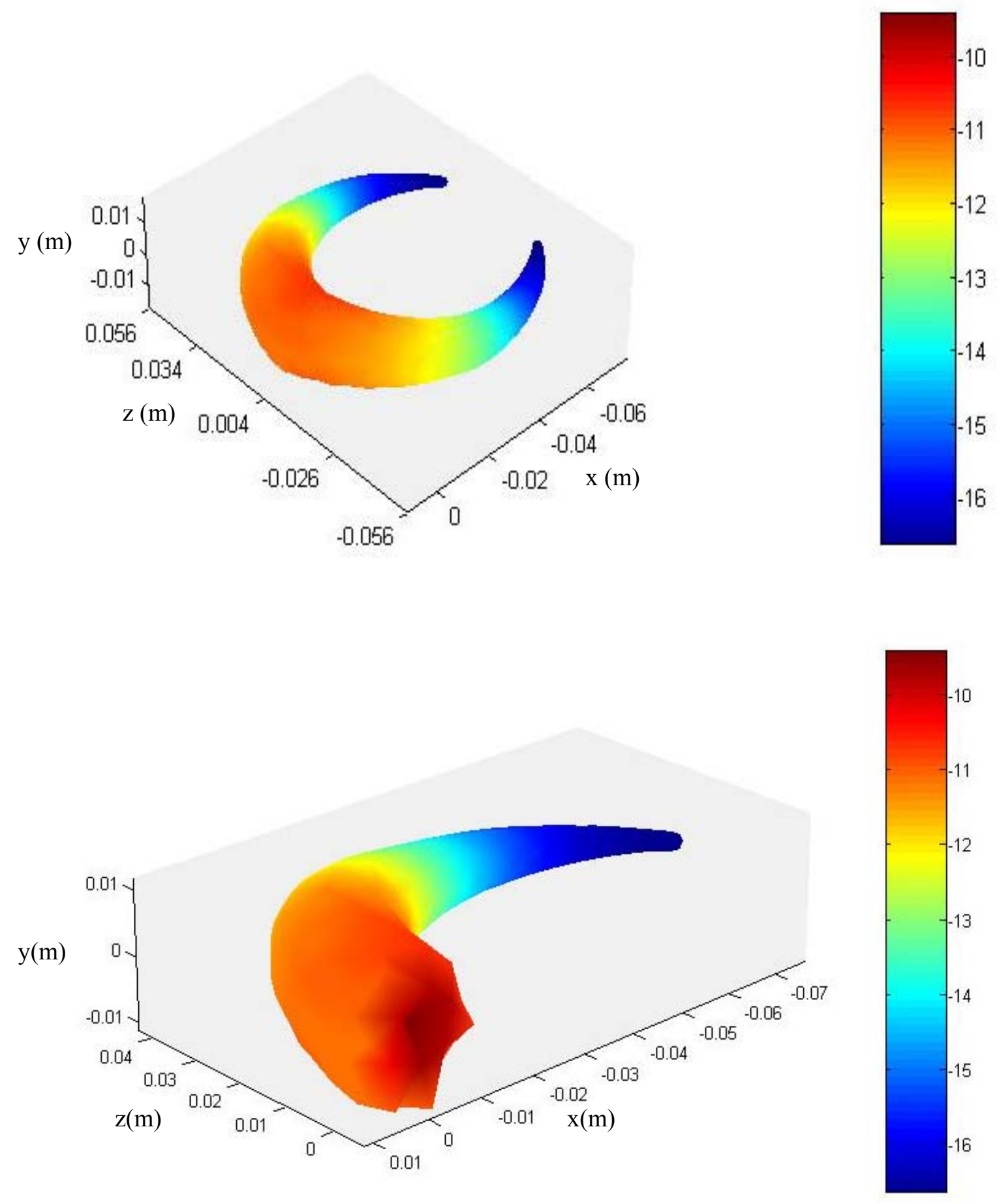

Fig. 6-9 a) Distribución de temperaturas en el alimento b) en un corte transversal sobre la sección más ancha, ambas luego de 25 minutos de comenzado el proceso de congelación 
Tabla 6-1 Tiempos de congelación (min) para distintos valores de $\mathrm{T}_{\text {ext }} \mathrm{y} \mathbf{h}$ (coeficiente de transferencia calórica)

\begin{tabular}{|lllllll|}
\hline \multicolumn{6}{c|}{ Coeficiente de transferencia calórica $\mathrm{h}\left(\mathrm{W} / \mathrm{m}^{2}{ }^{\circ} \mathrm{C}\right)$} \\
$\left.\mathbf{T}_{\text {ext }}{ }^{\circ} \mathrm{C}\right)$ & $\mathbf{5}$ & $\mathbf{1 0}$ & $\mathbf{1 5}$ & $\mathbf{2 0}$ & $\mathbf{4 0}$ & $\mathbf{5 0}$ \\
\hline-30 & 85.5 & 47 & 33.8 & 27 & 16.8 & 14.8 \\
-35 & 69 & 38 & 27.3 & 22 & 13.8 & 12.2 \\
-40 & 58.3 & 32.3 & 23.3 & 18.8 & 11.8 & 10.5 \\
\hline
\end{tabular}

En la Fig. 6-10 se observa la superficie de respuesta que pasa por los puntos generados en la Tabla 6-1 obtenidos a través de simulaciones realizadas en el código computacional.

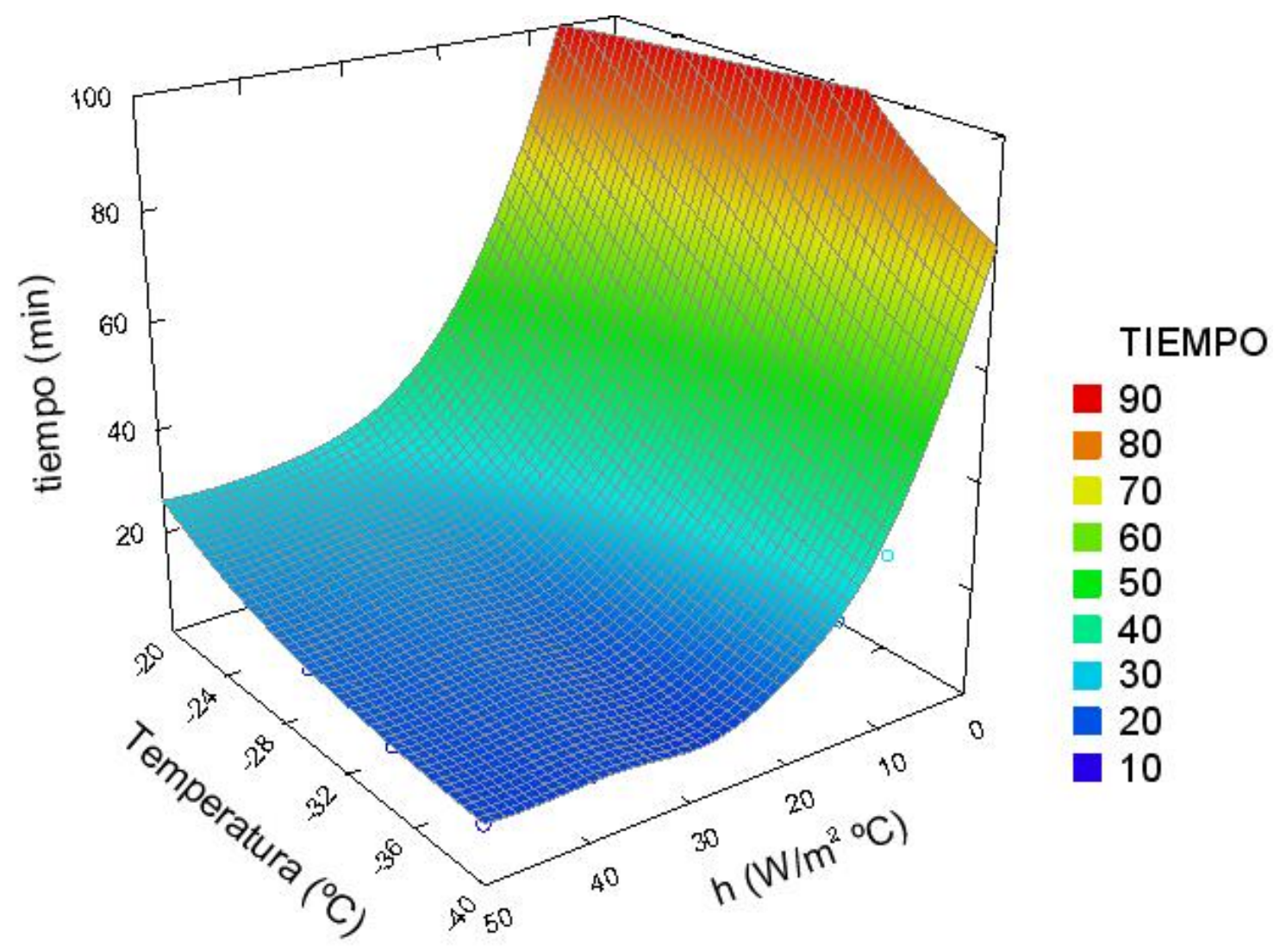

Fig. 6-10 Superficie representativa de los tiempos de congelación ( $\mathrm{min}$ ) versus temperatura y coeficiente de transferencia de calor en la interfase (h) para una $T_{0}=10^{\circ} \mathrm{C}$ 


\subsection{Conclusiones}

$>$ Se desarrolló un modelo matemático del proceso de congelación en productos panificados de geometría irregular tridimensional, utilizando el método de los elementos finitos realizando una simultánea transformación de variables en entalpía y función de Kirchhoff. Esta formulación permitió optimizar la velocidad de cálculo del código computacional convirtiendo el problema original no-lineal en uno lineal, donde las matrices globales son constantes.

$>$ Se determinaron experimentalmente el calor específico del producto en función de la temperatura y el coeficiente de transferencia de calor, los cuales fueron alimentados en la simulación numérica del problema.

$>$ El modelo fue validado experimentalmente obteniéndose una buena concordancia entre las historias térmicas predichas y las registradas en el laboratorio.

$>$ Se simularon historias térmicas para distintas condiciones de proceso (variando $\mathrm{T}_{\text {ext }} \mathrm{y}$ el coeficiente de transferencia calórica).

$>$ El modelo permite conocer los tiempos de congelación y la temperatura en cualquier punto del alimento utilizando un tiempo de ejecución de máquina mínimo (máximo 3 minutos), presentando la versatilidad de poder implementarlo en alimentos de geometría irregular con propiedades termofísicas variables con la temperatura. 
Capítulo 7 


\section{Conclusiones}

\subsection{Formulación matemática del MEF}

Se desarrollaron modelos numéricos que representan problemas de transferencia de calor utilizando una formulación variacional y el método de los elementos finitos. Estos modelos fueron implementados en sistemas con transferencia bi y tridimensional de geometría irregular no sólo con propiedades constantes sino también variables, generando los códigos numéricos en programas abiertos, los cuales permiten el acoplamiento de subrutinas específicas de interés para el usuario.

En el caso de sistemas bidimensionales de revolución se acoplaron balances macroscópicos de energía como también ecuaciones que representaban cinéticas de inactivación de microorganismos patógenos.

En dominios irregulares tridimensionales, donde la dificultad del planteo matemático se ve incrementada junto con la manipulación y visualización de un gran número de elementos tetraédricos que componen la malla, se desarrolló un programa computacional propio y las subrutinas específicas (pre- y postprocesamiento) para incorporar la información de la malla en el programa principal. De esta manera se logró obtener la temperatura en cualquier punto del dominio, la temperatura media, y flujo de calor en el dominio.

Los códigos computacionales fueron validados en todos los casos (dos y tres dimensiones) comparando las salidas numéricas con las soluciones analíticas en sistema de geometrías regulares (esfera y cilindro finito) y con datos de literatura.

Se logró la optimización de la velocidad de cálculo del programa utilizando varias subrutinas de Matlab, como ODE, que permiten resolver sistemas de ecuaciones diferenciales ordinarias.

Se estudió el problema de congelación desde el punto de vista matemático teniendo en cuenta la fuerte variación de las propiedades 
termofísicas del alimento con la temperatura y su influencia en la convergencia de los modelos numéricos aplicados. En estos sistemas complejos donde el problema es altamente no-lineal e interviene un cambio de fase, se profundizó en los conceptos matemáticos necesarios para obtener una solución aplicable a cualquier tipo de funcionalidad del calor específico y conductividad térmica del alimento con la temperatura. Para esto se desarrolló un modelo numérico aplicando la formulación entálpica y la de Kirchhoff, que implica el uso de una transformación de variables, resultando en un método estable que además permite reducir los tiempos de ejecución computacional.

Los programas generados son versátiles y pueden ser aplicados en productos alimenticios de geometrías diversas utilizando un generador de malla externo. Esto permite abordar un amplio espectro de problemas en la industria relacionados con la transferencia de energía en alimentos.

\subsection{Aplicaciones tecnológicas de los programas computacionales.}

Los modelos numéricos desarrollados fueron implementados para resolver diversos problemas tecnológicos de aplicación industrial.

\section{A. Aplicación del MEF al procesamiento térmico de alimentos acoplando cinéticas de destrucción microbiana}

El modelo numérico desarrollado fue aplicado de acuerdo a la necesidad del sector productivo de determinar condiciones de tiempo-temperatura que aseguren la inocuidad del producto cárnico (embutidos). El producto se consideró como un sistema bidimensional de revolución con contorno irregular. Se establecieron los requerimientos de tiempo-temperatura en el proceso de calentamiento, con el objetivo de lograr la inactivación térmica del microorganismo $E$. coli $0157: \mathrm{H} 7 \quad\left(\mathrm{VI}=12_{\mathrm{log}}\right)$ teniendo en cuenta 
condiciones de procesamiento industrial donde existe un descenso en la temperatura del fluido cuando se sumergen los productos. El modelo se basó en utilizar el balance microscópico diferencial de energía en estado no estacionario acoplando la cinética de destrucción microbiana y el balance macroscópico de energía. Se validó el modelo numérico realizando experimentos de laboratorio, mediante la medición de historias térmicas. En las simulaciones se consideró la relación carga de producto y la potencia de calefacción del sistema para la predicción del descenso de temperatura del fluido calefactor en función del tiempo. Se estudió el efecto de distintas relaciones de cargas y coeficientes de transferencia de calor determinándose los tiempos mínimos de proceso para alcanzar una reducción de $12_{\log }$ en la población microbiana.

\section{B. Simulación del proceso de refrigeración de alimentos con composición heterogénea de geometría irregular.}

El programa computacional desarrollado para geometrías complejas tridimensionales permitió simular problemas de transferencia de calor en sistemas heterogéneos (formados por más de un material, donde las propiedades térmicas eran distintas de acuerdo a la zona del dominio). El código computacional fue implementado para simular el proceso de enfriamiento de un producto semi-elaborado de interés para el sector industrial (empanadas). La simulación numérica del proceso permitió establecer condiciones adecuadas del proceso. Se determinaron experimentalmente el calor específico del producto mediante DSC, la densidad, el contenido de agua de cada uno de los materiales que componen el alimento y el coeficiente de transferencia calórica. Se estimó mediante modelos y correlaciones la conductividad térmica, la cual fue alimentada en el programa computacional. El modelo fue validado experimentalmente obteniéndose una buena concordancia entre las historias 
térmicas predichas y las registradas en cámaras de refrigeración. La simulación del proceso permitió conocer los tiempos mínimos de refrigeración, la temperatura en cualquier punto del alimento, y su valor medio en el dominio de cálculo. Se simularon historias térmicas para distintas condiciones de proceso (variando $T_{\text {ext }} y$ el coeficiente de transferencia calórica) analizándose el efecto de las distintas variables.

\section{Congelación de Alimentos. Problemas transferencia de calor con cambio de fase en sistemas tridimensionales de geometría irregular}

En el caso de problemas con cambio de fase el código computacional generado permitió simular el proceso de congelación de un producto de geometría irregular tridimensional utilizando un método combinado de transformación de variables para encarar el problema matemático altamente no-lineal. Se comprobó que existen dificultades para obtener la convergencia adecuada cuando se utiliza el calor específico aparente en las simulaciones mediante el MEF, tanto en los códigos numéricos propios como en paquetes comerciales de simulación. La formulación propuesta en el presente trabajo de Tesis, la cual consiste en utilizar un cambio de variables de la capacidad calorífica volumétrica y de la conductividad térmica resultó la más adecuada para abordar la problemática relacionada con las fuertes variaciones en las propiedades termofísicas durante la congelación. Se implementó el programa computacional codificado en Matlab para simular el proceso de congelación de un alimento panificado de geometría irregular (medialunas) dado el creciente desarrollo de tecnologías en las que los productos semiterminados congelados y/o enfriados son distribuidos a los puntos de ventas donde son horneados según su demanda. Se determinaron experimentalmente el calor específico del producto en función de la temperatura y el coeficiente de transferencia de calor, los cuales fueron alimentados al código numérico. Se comprobó la validez del modelo 
realizando experimentos, comparando las historias térmicas predichas y las registradas en el laboratorio. Se simularon historias térmicas para distintas condiciones de proceso (variando $T_{\text {ext }} \mathrm{y}$ el coeficiente de transferencia calórico) obteniéndose en cada caso la distribución de temperaturas.

La ventaja principal de esta formulación radica en la disminución en los tiempos de ejecución de máquina (máximo 3 minutos), por lo que se obtienen resultados exactos en tiempos cortos, pudiendo implementarse en alimentos cuyas geometrías no son regulares con propiedades termofísicas variables con la temperatura. 


\section{Referencias}

Abdalla, H. y Singh, R.P. (1985). Simulation of thawing foods using finite element method. Journal of Food Process Engineering 7, 273-286.

Adam, M., Celba, J., Havlicek, Z., Jeschke, J., Kubesova, A., Neumannova, J., Nesvadba, P., Pokorny, D., Sestak, J., y Sramek, P. (1997). Meat, meat products and semiproducts thermophysical and rheological properties of foods. Prague: Food Research Institute and Institute of Agricultural and Food Information.

Anónimo (2008). Opportunities still exists: EU ready-meals/ingredients market overview. Food Ingredients and Analysis International 27 (3), 4-6.

Arce, J.A., Potluri, P.L., Schneider, K.C., Sweat, V.E., y Dutson, T.R. (1983). Modeling Beef Carcasss Cooling Using Finite Element Technique. Transactions of the ASAE (American Society of Agricultural Engineer. Paper n 81-6030.

ASTM Standard E1269 - 05 "Standard Test Method for Determining Specific Heat Capacity by Differential Scanning Calorimetry", ASTM International, West Conshohocken, PA, 2003, DOI: 10.1520/E1269-05, www.astm. org.

Baik, O. D, Marcotte, M., Sabiani, S. S., y Castaigne, F. (2001). Thermal and Physical Properties of Bakery Products. Critical Reviews in Food Science and Nutrition 41 (5), 321-352.

Bathe, K.J (1996). Finite element procedures. New Jersey: Prentice Hall.

Becker, E., Carey, G.F., y Oden, J.T. (1983) Finite Elements: An Introduction (Vol. 1). Prentice-Hall, Englewood Cliffs, New Jersey. 


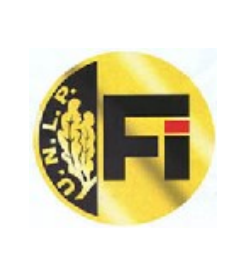

C I D C A

Belongia, E. A., MacDonald, K. L., Parham, G. L., White, K. E., Korlath, M. N., Lobato, S. M., Strand, S. M., Kasale, K. A., y Osterholm, M. T. (1991). An outbreak of Escherichia coli O157:H7 colitis associated with consumption of precooked meat patties. Journal Infection Disease 164, 338-343.

Blanco, J., Blanco, M., Escribano, A., Blanco, J. E., Alonso, M. P., Marín, J., y Hernández, R. (1995). Escherichia coli verotoxigénicos y el síndrome urémico hemolítico. Aspectos clínicos y microbiológicos. Anales Españoles de Pediatría 42, 9-19.

Califano, A. y Zaritzky, N. (1993). A Numerical Method for Simulating Heat Transfer in Heterogeneous and Irregular Shaped Foodstuffs. Journal of Food Process and Engineering 16 (3), 159-171.

Califano, A., Zaritzky N. (1997). Simulation of freezing or thawing heat conduction in irregular two dimensional domains by a boundary fitted grid method, Lebensmittel-Wissenschaft und-Technologie Vol. 30, 70-76.

Carslaw, H. S., y Jaeger, J.C. (1959). Conduction of heat in solids. University Press, Oxford.

CDC (2007). Centers for Disease Control and Prevention, Division of Bacterial and Mycotic Disease Department of Health and Human Services. http://www.cdc.gov/ncidod/dbmd/diseaseinfo/escherichiacoli_g.htm Accedido 10/04/07.

CFR (2009) Code of Federal Regulations, National Archives and Records Administration. 9 CFR Ch. III, 318.23, page 278.

Chang, H. D., y Tao, L. C. (1981). Correlations of enthalpies of food systems. Jounal of Food Science, 46, 1493-1497.

Chen, H., Marks, B.P., y Murphy, R.Y. (1999) Modeling coupled heat and mass 
transfer for convection cooking of chicken patties. Journal of Food Engineering, 42, 139-146.

Choi Y., y Okos M. R. (1986) Effects of Temperature and Composition on the Thermal Properties of Foods. In Le Magher and Jelen $\mathrm{P}$ Food Engineering and Processs Applications. Vol 1. New York. 93-103.

Cleland D.J.,Cleland A.C, Earle R.L., y Byrne S.J. (1984). Prediction of Rates of Freezing, Thawing or Cooling in Solids of Arbitrary Shape Using the Finite Element Method. International Journal of Refrigeration 7 (1), 6-13.

Cleland D.J.,Cleland A.C, Earle R.L., y Byrne S.J. (1987). Prediction of freezing and thawing times for multi-dimensional shapes by numerical methods. International Journal of Refrigeration 10, 32-39.

Código Alimentario Argentino. De la Canal y Asociados SRL. (2005), Alimentos Cárneos y Afines, Capítulo 6 Art.343.

Comini, G., Del Giudice, S, y Saro, O. (1989). Conservative equivalent heat capacity methods for non-linear heat cnduction. In: RW Lewis, K Morgan, Eds., Numerical Methods in Thermal Problems, Vol 6 Part 1. Swansea: Pineridge Press, 5-15.

Comini, G., Nonino, C., y Saro, O. (1990). Performance of enthalpy -based algorithms for isothermal phase change. Advanced Computational Methods in Heat Transfer, Vol 3: Phase Change and Combustion Simulation 3, 3-13.

COMSOL, AB (2005). COMSOL Multiphysics user's guide. Versión septiembre COMSOL 3.2.

Crank, J. (1984). Free and moving boundary problems. Oxford University Press. 
Dirección de Epidemiología, (2005). Diarrea aguda en Argentina. Precauciones especiales durante el período de verano. Epi-Noticias, número 527, año CV, 4, february 2005, Ministerio de Salud y Ambiente de la Nación, Argentina. www.direpi.vigia.org.ar/boletines/epinoticias/Destacado 0402-05.pdf Accedido 19/12/06.

Doyle, M. P. (1991). Escherichia coli O157:H7 and its significance in foods. International Journal of Food Microbiology, 12, 289-302.

Earl, R. L., (1988). Ingeniería de los Alimentos. (2 ${ }^{\text {da }}$ Ed.) Ed. Acribia, Zaragoza, España.

Erdogdu, F. (2005). Mathematical approaches for use of analytical solutions in experimental determination of heat and mass transfer parameters. Journal of Food Engineering, 68, 233-238.

FDA, Food and Drug Administration. (2009). Food Code. US Department of Health and Human Services. Annex 4 - Management of Food Safety Practices - Achieving Active Managerial Control of Foodborne IIIness RiskFactors.

http://www.fda.gov/Food/FoodSafety/RetailFoodProtection/FoodCode/Fo odCode2009/ucm188363.htm

Fennema O. R., Powrie, W. D., y Marth, E. H. (1973). Low Temperatures Preservation of Foods and Living Matter. Marcel Dekker, Inc. New York.

Fennema, O. W. (1993). Química de los alimentos, 2da. Edición. Editorial. Acribia, S. A. Zaragoza, España.

Fikiin, K. A. (1996). Generalized numerical modeling of unsteady heat transfer during cooling and freezing using an improved enthalpy method and quasi-one-dimensional formulation. Intl.J. Refrigeration 19(2): 132-140. 
Fikiin, K. A. (1998). Some general principles in modeling of unsteady heat transfer in two-phase multi-component aqueous food systems for product quality improvement. In Food Quality Modelling, B. M. Nicolaï and J. De Baerdemaeker, eds., 179-186. Luxembourg: Office for Official Publications of the European Communities.

Geankoplis, C. J. (1993). Transport Processes and Unit Operations. (3rd ed.) (pp. 259-262). New Jersey: Englewood Cliffs.

Holtz, E. y Skjoldebrand, C. (1986). Simulation of the temperature of a Meat Loaf During the Dooking Process. Jounal of Food Engineering, 5 (2), 109-121.

Hughes, T. J. R., (1987). The Finite Element Method- Linear Static and Dynamic Finite Element Analysis. Prentice-Hall, Englewood Cliffs, New Jersey,

Lamauro, C.J. y Bakshi, A.S. (1985). Finite element analysis of moisture diffusion in stored food Journal of Food Science 50-392-396.

Le Bail, A., y Goff, H. D., (2008). Freezing of Bakery and Dessert Products. In J. Evans, Frozen Food Science and Technology (pp.184-203). Oxford, UK: Blackwell Publishing.

Leithold, L., (1998). El Cálculo, $7^{\text {th }}$ ed. Oxford University Press-Harla México, México.

Levy, F. L., (1979). Enthalpy and specific heat of meat and fish in the freezing rate. Journal of Food Technology 14, 549-560.

Lind I.; (1991)The Measurement and Prediction of Thermal Properties of Food during Freezing and Thawing-A Review with Particular Reference to Meat and Dough. Journal of Food Engineering 13, 285-319. 
Mannapperuma, J. D., y Singh, R. P., (1988) Prediction o freezing and thawing times of foods using a numerical method based on enthalpy formulation. Jounal of Food Science 53, 626-630.

Mannapperuma, J. D., y Singh, R. P., (1989). A computer-aided method for prediction of properties and freezing/thawing times of foods. J. Food Eng. 9: 275-304.

Martins, R.C. (2006). Simple finite volumes and finite elements procedures for food quality and safety simulations. Journal of Food Engineering, 73, 327 $-338$.

McNaughton J.L. y Mortimer C.T. (1975) Calorimetría Diferencial de Barrido. Perking Elmer Corporation, Connecticut, USA.

Miles, C. A. (1974). Meat freezing-why and how? Proceedings meat Research Insitute Symposium No 3, bristol, 15.1-15.7.

Miles, C. A., van Beek, G., y Veerkamp, C. H. (1983) Calculation of the Thermophysical Properties of Foods. In R. Jowitt et al.Physical Properties of Foods (Eds.) 269-312. London: Appl. Sci. Publ.

Miliwebsky, E. S., Balbi, L., Gómez, D., Wainsztein, R., Cueto Rua, M., Roldan, C., Calleti, M., Leardini, A., Baschkier, A., Chillemi, G. M., y Rivas, M. (1999). Síndrome urémico hemolítico en niños de Argentina: asociación por infección con Escherichia coli productor de toxina shiga. Bioquímica y Patología Clínica, 63, 113-120.

N. Graiver, A. Pinotti, A. Califano, y N. E. Zaritzky (2007) Modelado matemático del proceso de curado húmedo de carne. Actas del XI Congreso Argentino de Ciencia y Tecnología de Alimentos (CYTAL XI), 12 al 14 de setiembre 2007, Buenos Aires (CD). 
Naveh, D., Kopelman, L.J. y Pflug, I.J. (1983). The finite element method in thermal processing of foods. Journal of Food Science 48, 1086-1093.

Nesvadba P., (2008). Thermal Properties and Ice Crystal Development in Frozen Foods. In J. Evans, Frozen Food Science and Technology (pp.125). Oxford, UK: Blackwell Publishing.

Nicolai, B., van den Broek, P., Schellekens, M., De Roeck, G., Martens, T. y Baerdemaeker, J. (1995). Finite element analysis of heat conduction in lasagna during thermal processing. International Journal of Food Science and Technology, 30, 347-363.

Oteiza, J. M., Chinen, I., Miliwebsky, E., y Rivas, M. (2006). Isolation and characterization of shiga toxin-producing Escherichia coli from precooked sausages (morcillas). Food Microbiology, 23, 283-288.

Oteiza, J.M., Giannuzzi, L. y Califano, A.N. (2003). Thermal inactivation of Escherichia coli 0157:H7 and Escherichia coli isolated from morcilla as affected by composition of the product, Food Research International 36 (7) $703-712$.

Ou, D. \& Mittal, G. S. (2006). Double-sided pan-frying of unfrozen/frozen hamburgers for microbial safety using modelling and simulation. Food Research International 39, 133-144.

Pan, Z., Singh R. P., \& Rumsey T. R. (2000). Predictive modeling of contactheating process for cooking a hamburger patty. Journal of Food Engineering 46, 9-19.

Pearson A.M., y Gillet T.A. (1996). Processed Meats. Chapman y Hall. New York, Estados Unidos.

Persson, P.O., y Strang G., (2004). A Simple Mesh Generator in MATLAB.SIAM 
Review, 46 (2): 329-345.

Pham, Q.T, (1991). Shape Factors for the Freezing Time of Ellipses and Ellipsoids. Journal of Food Engineering, 13, 159-170.

Pham, Q.T, (1995). Comparison of general purpose finite element methods for the Stefan problem. Numerical Heat Transfer Part B -Fundamentals 27:417-435.

Pham, Q.T, (2006). Mathematical modeling of freezing processes. In Da-Wen Sun, Handbook of frozen food processing and packaging (pp.141-173). New York: Taylor \& Francis Group.

Pham, Q.T, (2008). Modelling of Freezing Processes. In J. Evans, Frozen Food Science and Technology (pp.51-80). Oxford, UK: Blackwell Publishing.

Rabellato, L., Del Giudice, S. y Comini, G. (1987). Finite element analysis of freezing process in foods. Journal of Food Science 43, 239-243-250.

Rabellato, L., Del Giudice, S. y Comini, G., (1978). Finite element analysis of freezing process in foodstuffs. Journal of Food Science 43, 239-250.

Rahman, M. D. (1995). Food Properties Handbook. CRC Press, BocaRatón, Florida.

Rahman, M. S., Shyam S. S., Al-Habsi, N., Almaskri, S, y Al-Belushi, R., (2005). State Diagram of Freeze-dried Garlic Powder by Differential Scanning Calorimetry and Cooling Curve Methods. Journal of Food Science 70 (2), 135-141.

Riley, L. W. (1987). The epidemiologic, clinical, and microbiologic features of haemorragic colitis. Annual Review of Microbiology, 41, 383-407. 
Rodriguez, N., y Zaritzky, N.E. (1986). Modeling of Sulfur Dioxide Uptake in Pre-Peeled Potatoes of Different Geometrical Shapes. Journal of Food Science, 51(3), 618-622.

Sá M. M., Figueiredo A. M., Correa A., y Sereno A. M., (1994) Apparent heat apacities, initial melting points and heats of melting of frozen fruits measured by differential scanning calorimetry. Revista Española de Ciencia y Tecnología de Alimentos, 34 (2), 202-209.

Salvadori, V. O., y Mascheroni, R. H., (1991). Prediction of Freezing and thawing times of foods by means of a simplified analytical method. Journal of Food engineering, 13, 67-68.

Saro O., Nonino, C., y Comini G; (1995) An enthalpy-based algorithm for the analysis of phase change in nonhomogeneous media. In Computational Modelling of Free and Moving Boundary Problems III, 127-134. ed. L.C. Wrobel, B. Sarler y C. A. Brebbia, Southampton, U.K.: Computational Mechanics Publications.

Scheerlinck N., Verboven P., Fikiin K. A., De Baerdemaeker J. y Nicolaï B M. (2001). Finite element computation of unsteady phase change heat transfer during freezing or thawing of food using a combined enthalpy and Kirchhoff transform method. Transactions of the ASAE (American Society of Agricultural Engineers): 44(2), 429-438.

Segerlind, L. J., (1984). Applied Finite Element Analysis, 2nd ed. John Wiley and Sons, New York.

Shampine L.F. (2005); Error Estimation and Control for ODEs. Journal of Scientific Computing, 25(1), 3-16.

Singh R. P. y Heldman D. R., y (1993) Introducción a la Ingeniería de los 
Alimentos. Editorial Acribia, Zaragoza, España.

Sweat, V. E., (1975). Trans. ASAE, 564.

Tocci, A. M., y Mascheroni, R. H., (1995). Numerical models for the simulation of the simultanous heat and mass transfer during food freezing and storage. International Communications in Heat and Mass Transfer, 22 (2), 251-260.

van der Sman, R. G. M, y Boer, E. (2005). Predicting the initial freezing point and water activity of meat products from composition data. Journal of Food Engineering, 66, 469-475.

Weast, R. C., y Astle, M.J., (1981). Handbook of chemistry and physics. 63 Ed. Boca Raton, Florida: CRC press.

Welty, J. R. (1974). Engineering Heat Transfer. John Wiley and Sons, New York,.

Zienkiewicz, O.C., y Taylor, R.L. (1994) a) El método de los elementos finitos, volumen I. McGraw-Hill, Barcelona.

Zienkiewicz, O.C., y Taylor, R.L. (1994) b)El método de los elementos finitos, volumen II. McGraw-Hill, Barcelona. 
$1 \quad$ Introducción 2

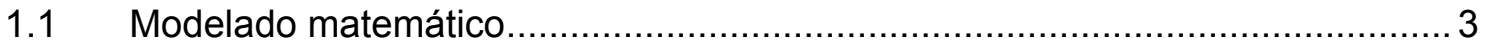

$1.2 \quad$ Problemática planteada desde el sector industrial ...................................... 4

1.3 Problemas de transferencia de calor sin cambio de fase (problemas lineales).

1.4 Problemas de transferencia de calor con cambio de fase (problemas no lineales).

1.4.1 Propiedades térmicas durante el cambio de fase (temperatura inicial de congelación).

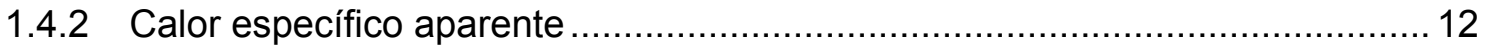

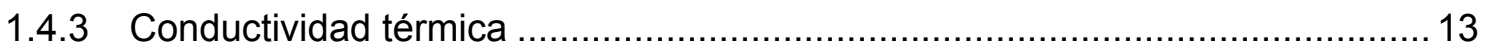

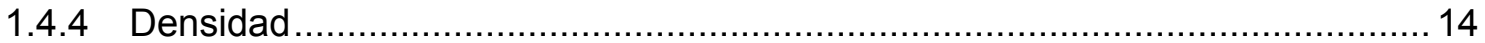

1.4.5 Resolución numérica de problemas de conducción de calor con cambio de fase

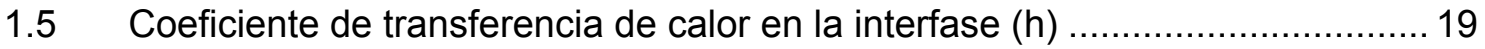

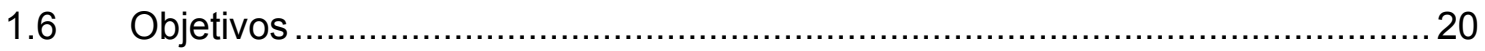

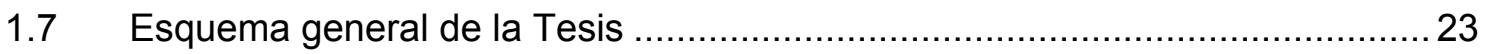

Capítulo 2

2 Resolución de problemas de transferencia de calor mediante MEF en dos dimensiones

2.1 Problemas lineales en dos dimensiones (2D) ................................................ 26

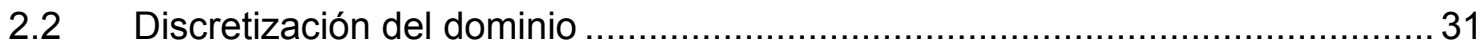

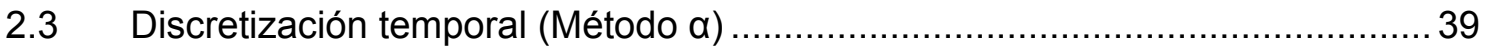


2.4 Ensamble de las matrices elementales ....................................................... 41

2.5 Desarrollo del programa computacional en elementos finitos ........................ 42

2.6 Validación del programa computacional en problemas de transferencia de calor en sistemas bidimensionales con propiedades termofísicas constantes.

2.6.1 Simulación de problemas con condición de borde tipo Dirichlet (temperatura prescripta en el borde) .....

2.6.2 Simulación de problemas con condición de borde convectiva 51

2.7 Conclusiones del Capítulo 54

Apéndice 2-1 55

Código Computacional 2D desarrollado en Matlab 6.5 55

2.7.1 Preprocesamiento .55

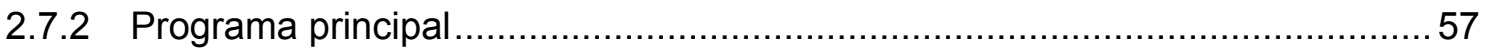

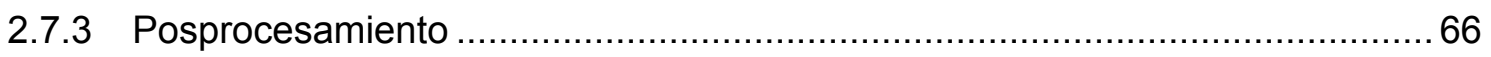

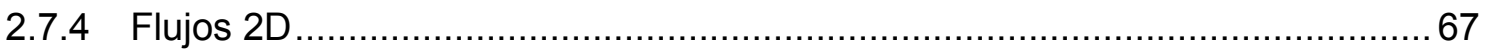

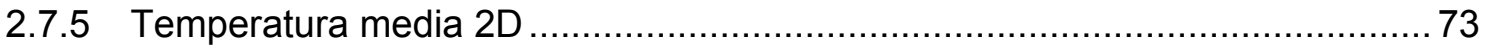

Capítulo 3

3 Aplicación del MEF al procesamiento térmico de alimentos acoplando cinéticas de destrucción microbiana $\quad 77$

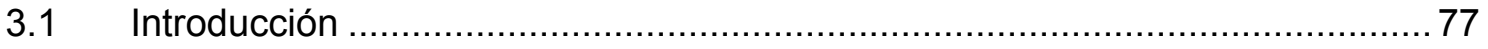

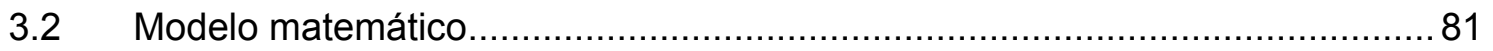

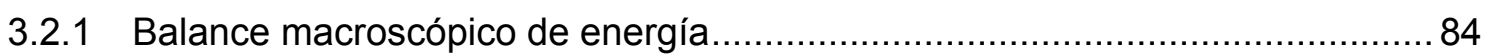

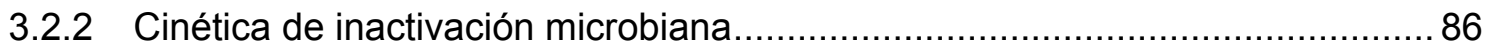

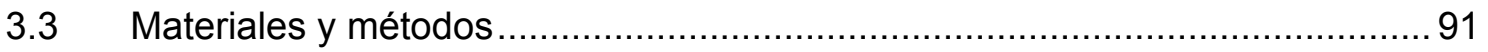


3.3.1 Procedimiento para la validación experimental del balance microscópico de energía durante el calentamiento considerando temperatura de fluido constante

3.3.2 Determinación del coeficiente de transferencia de calórica en el baño termostático

3.3.3 Determinación del calor efectivo del quemador

3.3.4 Experimentos para validar el descenso de temperatura del fluido de calentamiento en función de la relación de carga $(\mathrm{RC})$ modelado de acuerdo 3.2 .1

3.3.5 Aplicaciones del modelo numérico desarrollado para predecir tiempos de procesamiento variando condiciones operativas

3.4 Resultados y discusión 94

3.4.1 Coeficiente de transferencia calórica en la interfase. 94

3.4.2 Validación del proceso de calentamiento del producto cárnico utilizando una temperatura de fluido constante.

3.4.3 Validación del balance macroscópico de energía acoplado al balance microscópico para evaluar el descenso de temperatura del fluido calefactor

3.4.4 Efecto del descenso de la temperatura del agua en la inactivación térmica del microorganismo E. coli O157:H7

3.4.5 Predicción de tiempos mínimos de proceso para distintas condiciones operativas.

3.5 Conclusiones 102 
$4 \quad$ Resolución de problemas de transferencia de calor mediante el MEF en tres dimensiones

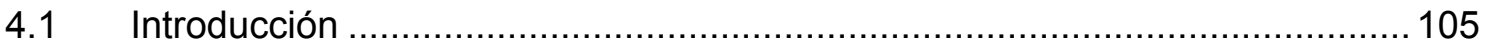

4.2 Generación de la malla y preprocesamiento ............................................... 107

4.3 Programa en elementos finitos ................................................................ 110

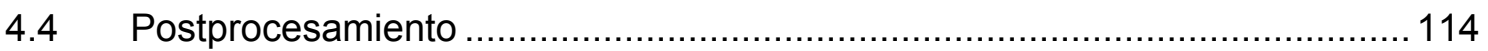

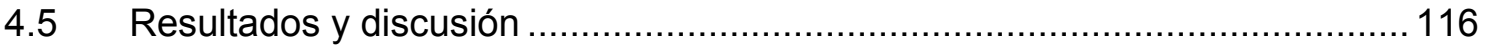

4.5.1 Validación del código computacional mediante solución analítica para esfera.

4.5.2 Validación del código computacional mediante solución analítica para cilindro finito.

4.5.3 Validación del código computacional en una geometría 3D irregular mediante un software comercial de elementos finitos.

4.6 Conclusiones 126

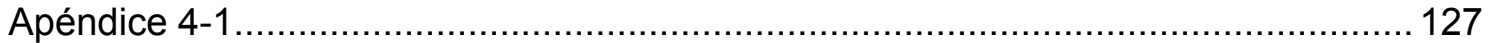

Código Computacional 3D desarrollado en Matlab 6.5 …....................................... 127

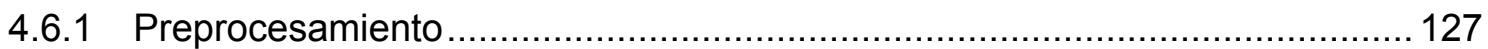

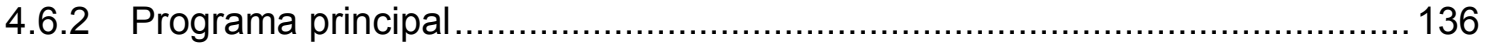

5 Simulación del proceso de refrigeración de alimentos con composición heterogénea de geometría irregular 3D utilizando el MEF 150

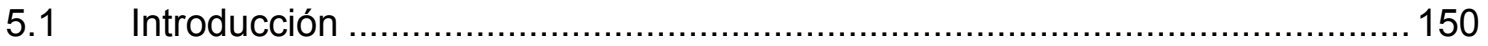

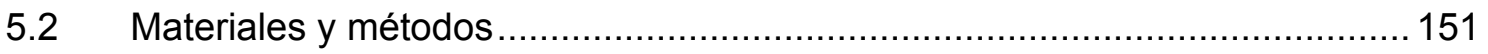

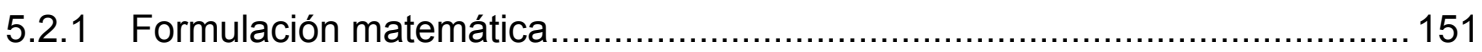

5.2.2 Optimización del código computacional................................................... 151 
5.2.3 Sistema experimental para validar el modelo numérico 152

5.2.4 Generación de la malla 153

5.2.5 Determinación experimental de las propiedades termofísicas del producto (calor específico aparente y densidad).

5.2.6 Determinación experimental del coeficiente de transferencia de calor en la interfase $(h)$.....

5.2.7 Aplicación del modelo numérico para predecir tiempos de refrigeración para distintas condiciones de proceso......

5.3 Resultados y discusión 156

5.3.1 Análisis de la malla utilizada para modelar la geometría irregular. 156

5.3.2 Propiedades termofísicas de las fases (cárnea y farinácea) que componen el alimento

5.3.3 Coeficiente de transferencia calórica en interfase 161

5.3.4 Validación experimental del modelo 161

5.3.5 Aplicación del modelo en la predicción de tiempos de enfriamiento para distintas condiciones de proceso

5.4 Conclusiones 164

Capítulo 6

6 Simulación de problemas de transferencia de calor con cambio de fase en sistemas tridimensionales de geometría irregular utilizando el MEF 166

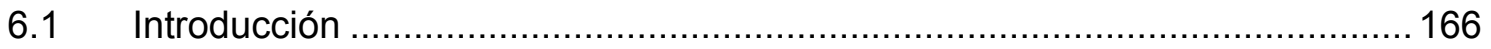

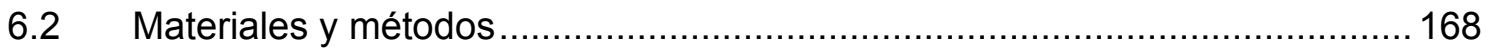

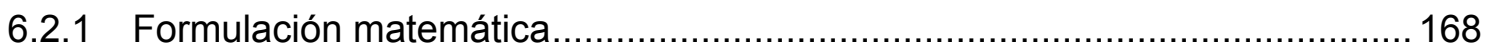

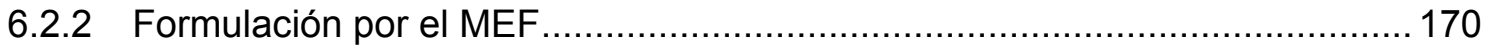

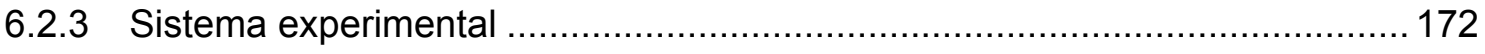

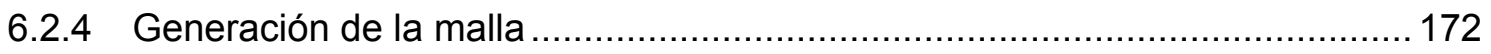


6.2.5 Determinación experimental del calor específico y del coeficiente de transferencia de calor en la superficie.

6.2.6 Aplicación del modelo numérico para predecir tiempos de congelación bajo distintas condiciones de proceso

6.3 Resultados y discusión

6.3.1 Análisis de la malla utilizada para modelar la geometría irregular. 174

6.3.2 Propiedades termofísicas 175

6.3.3 Coeficiente de transferencia calórica (h) 180

6.3.4 Validación experimental del modelo 181

6.3.5 Cálculo de tiempos de congelación para distintas condiciones de proceso

6.4 Conclusiones 186

Capítulo 7 187

$7 \quad$ Conclusiones

7.1 Formulación matemática del MEF 188

7.2 Aplicaciones tecnológicas de los programas computacionales. 189

$8 \quad$ Referencias 